\title{
4 a
}

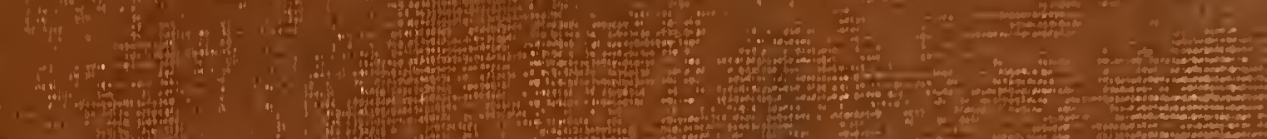

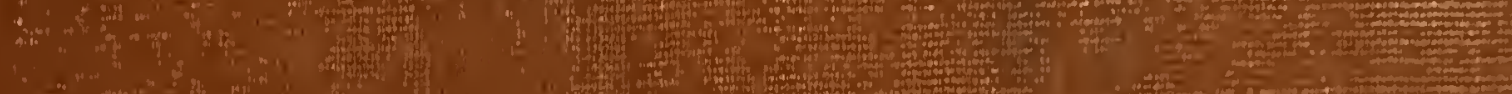

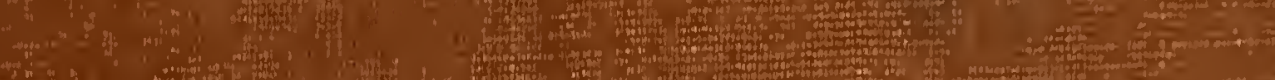

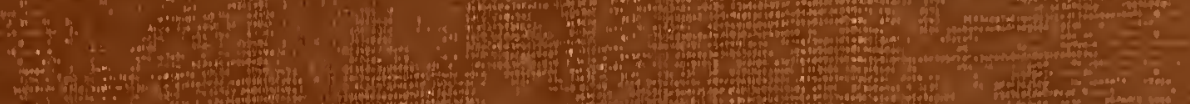
if

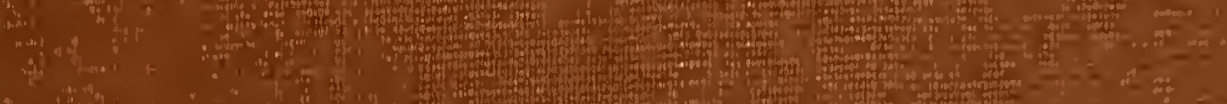

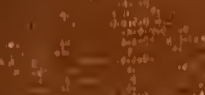

\section{-}

$(4)$

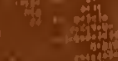
(15) 7int

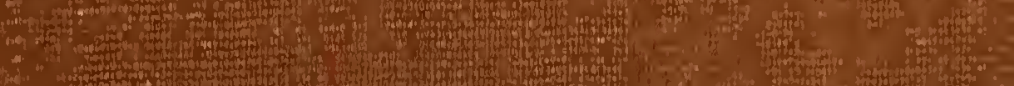

a.t.

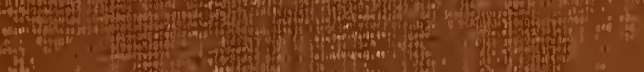
fimbing

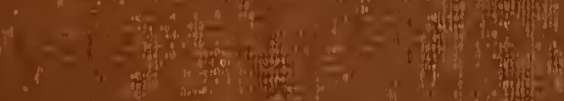

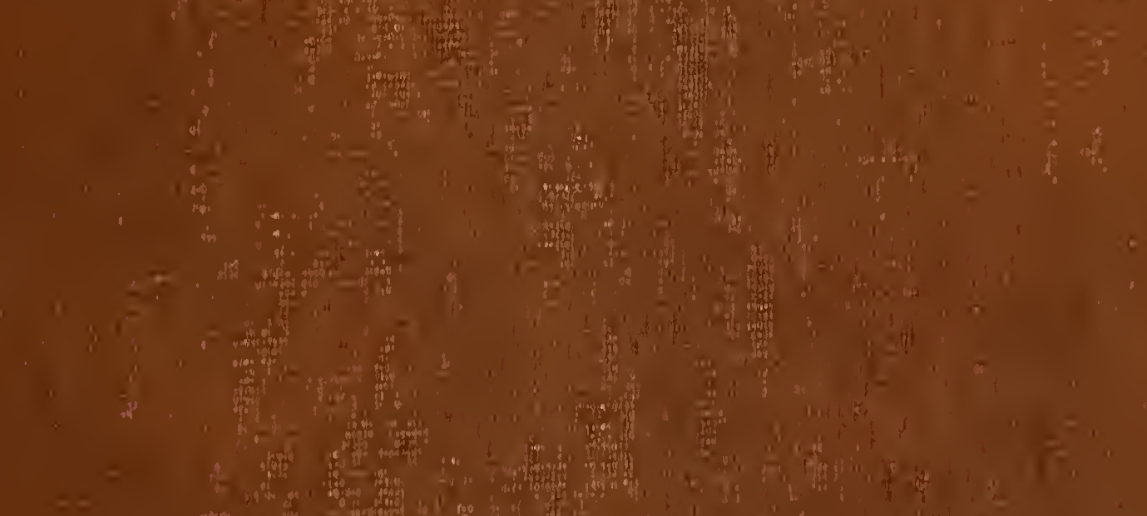
$\sin 20$

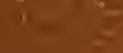





\section{INDEX OF PATENTS}

RELATING TO

\section{ELECTRICITY,}

ERBRACING PATENTS ISSUED BY

\section{THE UNITED STA'TES PATEN'T OFFICE}

FROM JULY 1, 1890, T0 JUNE 30, 1891.

\section{APPENDIX No. X.}

COMPILED UNDER THE DIRECTION OF THE COMMISSIONER OF PATENTS.

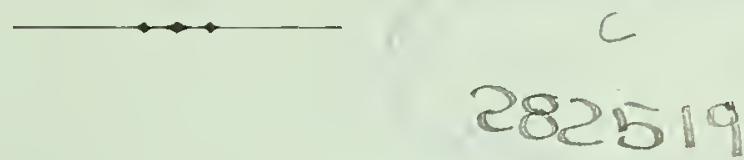

WASHINGTON:

GOVERNMENT PRINTING OFFICE.

1892. 



\section{INDEX OF PATENTS}

RELATING TO

\section{ELECTRICITY,}

EMBRACING PATENTS ISSUED BY

\section{THE UNITED STATES PATENT OFFICE}

FROMI JULY 1, 1890, T0 JUNE 30, 1891.

\section{APPENDIX No. X.}

COMPILED UNDER THE DIRECTION OF THE COMMISSIONER OF PATENTS.

WASHINGTON:

GOVERNMENT PRINTING OFFICE.

1892. 



\section{APPENDIX No. X.}

\section{PARTI.}

CONSISTING OF A

\section{SUB-CLASS INDEX OF ALL PATENTS}

RELATING TO

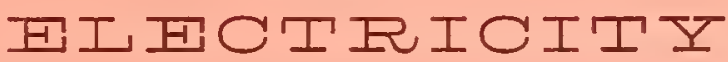

Issued from July 1, 1890, to June 30, 1891, inclusive, giving names of Inventors, and lates and numbers of Patents in each sub-class in numerical and chronological order. 



\title{
CLASSIFIED INDEX
}

OF

\section{Patents Relating to Electricity}

\author{
From July 1, 1890 , TO JunE 30, 1891.
}

17. GENERATION.-6. Batteries-Galvanic.

\begin{tabular}{|c|c|c|c|c|c|}
\hline No. & Name. & DATE. & No. & NAME. & Date. \\
\hline $\begin{array}{l}431,073 \\
432,681 \\
432,973 \\
433,738 \\
434,593 \\
435,346 \\
435,421 \\
435,685 \\
436,001 \\
436,516 \\
437,393 \\
437,469 \\
438,586 \\
439,151 \\
439,516 \\
439,737 \\
440,420 \\
441,130 \\
441,403 \\
441,843 \\
441,958 \\
441,967 \\
442,336\end{array}$ & 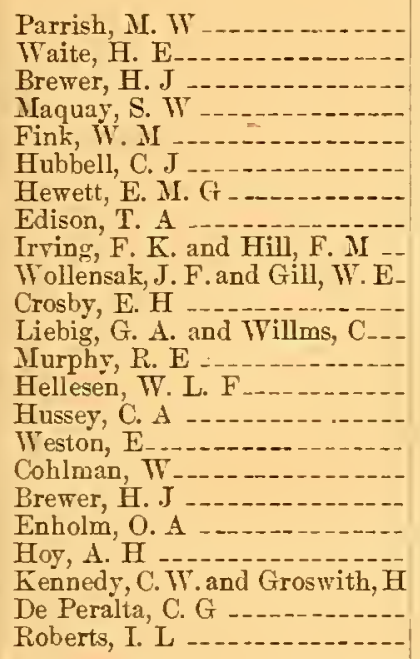 & $\begin{array}{l}\text { July } 1,1890 \\
\text { July } 22,1890 \\
\text { July } 29,1890 \\
\text { Aug. } 5,1890 \\
\text { Aug. } 19,1890 \\
\text { Aug. 26, } 1890 \\
\text { Sept. 2, } 1890 \\
\text { Sept. } 2,1890 \\
\text { Sept. } 9,1890 \\
\text { Sept. } 16,1890 \\
\text { Sept. } 30,1890 \\
\text { Sept. } 30,1890 \\
\text { Oct. } 14,1890 \\
\text { Oct. 28, } 1890 \\
\text { Oct. } 28,1890 \\
\text { Nov. } 4,1890 \\
\text { Nov. } 11,1890 \\
\text { Nov. } 25,1890 \\
\text { Nov. 25, } 1890 \\
\text { Dec. } 2,1890 \\
\text { Dec. } 2,1890 \\
\text { Dec. } 2,1890 \\
\text { Dec. } 9,1890\end{array}$ & $\begin{array}{l}442,516 \\
443,219 \\
443,976 \\
445,425 \\
446,544 \\
446,727 \\
447,016 \\
448,798 \\
448,847 \\
448,936 \\
449,299 \\
450,105 \\
450,285 \\
450,354 \\
450,840 \\
451,142 \\
451,948 \\
452,303 \\
453,893 \\
454,057 \\
454,598 \\
454,724\end{array}$ & 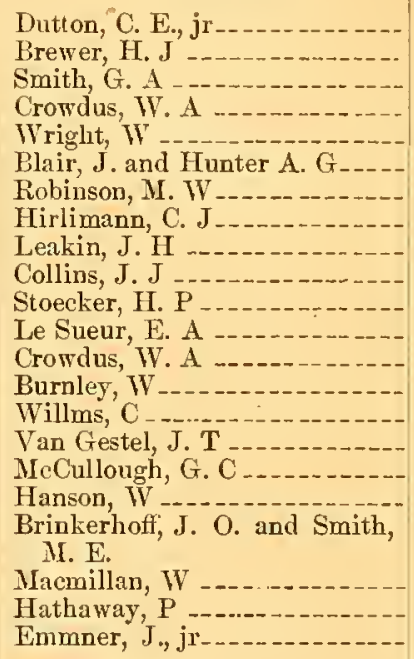 & 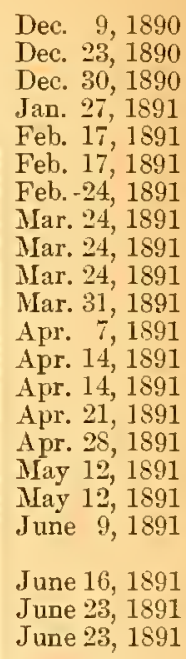 \\
\hline
\end{tabular}

171. GENERATIOX.-72. Batteries-Secondary.

431,341 Foote, T. M

431,447

431,742

431,968

432,202

432,672

432,834

433,174

434,093

434,199

431,224

434,301

434,376

434,444

434,457

434,458

434,869

436,050

436,602

437,848

438,532

438,827

439,240

439,301

439,324

439,416

435,594

$439,8.50$

440,023

Woolf, A. E

\section{Dixon, T. S. E}

Goodwin, C. $R$

Goodwin, C. R

McLaughlin, J. F

Eookogey, WW.

Morris, H. G

Kookogey, W. P

Schoop, P -................

Pumpelly, J. K

TToodward, $\mathrm{H}$

Schoop, P - - Cochran, G. W

Hicks, T. H

Pepper, D, jr Pepper, D., jr

Hollingshead, W. B

Whittier, T. P Hollingshead, $\overline{\mathrm{W}} . \overline{\mathrm{B}}$ Lampert, $H_{\text {., }} \mathbf{j r}$ Currie, S. C. C Gibson, C. D. P

Mehren, J. F

Morrison, W. and Schmidt, L-

Hollingshead, W. B ........

Entz, J B and Phillips, W A

July 1,1890 July 1,1890 July 8,1890 July 8, 1890 July 15,1890 July 22, 1890 July 22, 1890 July 29,1890 Aug. 12, 1890 Aug. 12, 1890 Aug. 12, 1890 Aug. 12, 1890 Aug. 12, 1890 Aug. 19, 1890 Aug. 19, 1890 Aug. 19, 1890 Aug. 19, 1890 Sept. 9, 1890 Sept. 16, 1890 Oct. 7,1890 Oct. 14,1890 Oct. 21,1890 Oct. 28,1890 Oct. 28, 1890 Oct. 28,1890 Oct. 28,1890 Oct. 28,1890 Nov. 4, 1890 , B. and Phillips, W. A. Nov. 4, 1890
440,024 440,175 440,210 440,216 440,505

441,413

441,818

441,959

442,187

442,203

442,204

442,390

442,391

442,969

443,454

443,455

443,456

443,457

443,556

444,392

444,969

445,422

445,542

445,872

446,104

446,527

447,279
Entz, J. B. and Phillips, IV. A Marx, $\mathrm{F}$.

Weed, E. B

Woolf, A. E _............

Renault, B. and Desvernay, M

Hatch, G. E

Bradbury, J. Y. and Stone, F. J.

Kennedy, C. W

Johnston, J. G

Roberts, I. L

Roberts, I. I

Pumpelly, J. K

Pumpelly, J. K

Fitz-Gerald, D. G. and Hough, A. H.

Kitsee, I

Kitsee, I .

Kitsee, I _...................

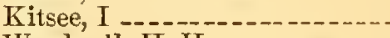

Wardwell, H. H

Currie, S. C. C

Bain, F

Brush, C. F

Cheswright, H. T

Barrett, S. H

M Laurent-Cély, A. M. F. and Feb. 17, 1891

Timmis, I. A.

Currie, S. C. C
Nov. 4, 1890 Nov. 11,1890 Nov. 11, 1890 Nov. 11, 1890 Nor. 11,1890 Nov, 25, 1890 Dec. 2, 1890 Dec. 2, 1890 Dec. 9,1890 Dec. 9,1890 Dec. 9,1890 Dec. 9,1890 Dec. 9, 1890 Dec. 16,1890

Dec. 23, 1890 Dec. 23,1890 Dec. 23, 1890 -e. 23,1890 Dec. 30,1890 Jan. 6,1891 Jan. 20, 1891 Jan. 27, 1891 Feb. 3, 1891 Feb. 24, 1891 
171. GENERATION.-33. MAGNeto-Electric.

\begin{tabular}{|c|c|c|c|c|c|}
\hline Yo. & & Date. & No. & E. & DAte. \\
\hline & 1 & aly 22,1890 & 60 & dgerton, $\mathrm{N}$ & Dec. 16,1890 \\
\hline & & 90 & & . & Dec. 23, \\
\hline & $\mathrm{I}$ & July 2 & & e, & Jan. 6,1891 \\
\hline & H. Y & July 29, 1890 & 6 & Entz, J. B & Jan. 13, 1891 \\
\hline & שית & July 29, 1890 & 4 & Cook, A. S & Jan. 13,1891 \\
\hline & lding, $\Pi$. & July 29, 1890 & 1 & Condiet, $\mathrm{G} . \mathrm{H}$ & Jan. 27, 1891 \\
\hline & ng, W. & July 29,1890 & 96 & varze, C. & Feb. 3,1891 \\
\hline & I & July 29,1890 & & , E. K & Feb. 10,1891 \\
\hline & J. I & Aug. 5,1890 & 6 & hall, $\mathrm{H}$. & Feb. 17,1891 \\
\hline & , C. & Aug. 5, 1890 & 2 & E. W., & Mar. 3, 1891 \\
\hline & & g. 12,1890 & & lomson, E & Mar. 3,1891 \\
\hline & B. & g. 19, & & ain, $\mathrm{W}$ & Mar. 3, 1891 \\
\hline & c & 90 & & $N$ & Mar. 10,1891 \\
\hline & u, $\mathbf{D}$ & Aug. 26, & & bergel; & Mar \\
\hline & A. & Sept. 2, & & , K. G & Mar \\
\hline & & t. 2 , & & S. E & Ma \\
\hline & T. $A$ & Sept. 2, 1890 & & F. A & Mar. 24, 1891 \\
\hline & D & Sept. 16, 1890 & & $\mathrm{el}, \mathrm{H}$ & Apr. 7,1891 \\
\hline & & & & V. F & Apr. 14, \\
\hline & & Sept. 3 & & $\mathrm{er}, \mathrm{R}$ & Apr. 14, 1891 \\
\hline & 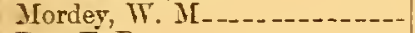 & Sept. 30,1890 & 2 & La M. & Apr. 14,1891 \\
\hline & $\mathrm{B}$ & Sept. 30,1890 & & g, J. & Apr. 21, 1891 \\
\hline & H. & Oct. & & A. L & Apr. 21, 1891 \\
\hline & $t$ & Oet. 7, & & & Apr. 28, 1891 \\
\hline & & Oct. 14, & & c & Apr. 28, 1891 \\
\hline & & Oct. 21 & & $\mathrm{C}_{-}$ & Мay 5,1891 \\
\hline & & Oct. 21 & & $\mathbf{T}$ & May 5,1891 \\
\hline & & Nor. 4, 1890 & & , S. S & May 5,1891 \\
\hline & $\mathrm{F}$ & $.11,1890$ & & W. F & May 5,1891 \\
\hline & & & & H. II & May 12,1891 \\
\hline & & 90 & & 1 & May 19,1891 \\
\hline & & & & J. M & Мay 19,1891 \\
\hline & $\mathrm{R}$ & Nov. 18,1890 & & mew, I. H & May 19,1891 \\
\hline & $\begin{array}{l}\text { Andersen, F. V. and Girdle- } \\
\text { stone, J. O. }\end{array}$ & Nov. 18,1890 & $\begin{array}{l}452,717 \\
452,718\end{array}$ & $\begin{array}{l}\text { Rike } \\
\text { Rik }\end{array}$ & $\begin{array}{l}\text { May } 19,1891 \\
\text { May } 19,1891\end{array}$ \\
\hline & $\mathrm{Lie}$ & Nov. 25,1890 & 452,934 & n, IV. K & May 26,1891 \\
\hline & & & & , E $\mathbf{E}_{--}$ & May 26, 1891 \\
\hline & B & Nov. 25 & & & June 9,1891 \\
\hline 87 & Harrington, W. E & Nov. 25, 1890 & 453,858 & Davis, H. and Sto & June 9,1891 \\
\hline 411,695 & Patterson, W. S & Dec. $\quad 2,1890$ & & Eickemeyer, R -- & June 16, 1891 \\
\hline $\begin{array}{l}441,793 \\
412,173\end{array}$ & & $\begin{array}{l}\text { Dec. } \quad 2,1890 \\
\text { Dec. } \\
9,1890\end{array}$ & 454,488 & Licb, C & June 23,189 \\
\hline & & & & & \\
\hline
\end{tabular}

171. GENERATION.-118. MagNeto-Electric-CoUplings.

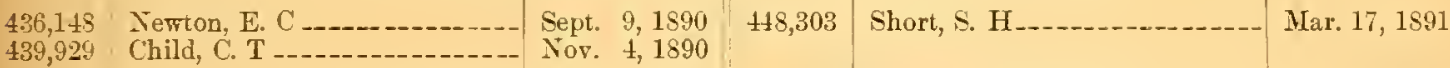

171. Generation.-76. Magneto-Electric-Hand Machines.

431,5.59 Colgate, A. E _._. 438,434 Ginocbio, X. B........... Oet. 14, 1890 453,242 Humans, W .............. June 2, 1891

\section{GENERATION.-34. METERs.}

July 1,1890 July 15,1890 July 22, 1890 Aug. 19, 1890 Sept. 30, 1890 Oct. 7,1890 Oct. 7,1890 Oct. 28, 1890 Nov. 11, 1890 Nov. 18,1890 Dec. 9,1890 Dec. 16,1890 Dec. 30,1890 Dec. 30, 1890 Jan. 20, 1891 Jan. 20, 1891
448,280 Thomson, E

448,894 Thomson,

448,676 Shallenberger, O. B..........

Ieston, A. IV

Elieson, C. P

Thomson, E

Thomson, E
448,677

449,001

449,002

449,003

451,125

451,520

452,276

452,935

452,936

Shallenberger, $\mathrm{O}$. $\mathrm{B}$

Shallenberger, O. B

Shallenberger, O. B

Shallenberger, O. B

Irving, F. K

Rice, $\mathrm{E}$, iv jr

Liller, H. WW

Freeman, W. $\mathrm{K}$

Pilkington, H. M. and White, June 30, 1891 R. S.

455,068 Tesla, $\mathrm{N}$
Mar. 17,1891

Mar. 24, 1891 Mar. 24, 1891 Mar. 24, 1891 Mar. 24, 1891 Mar. 24, 1891 Mar. 24, 1891 Apr. 28, 1891 May 5, 1891 June 30,1891 June 30, 1891 
171. Generation.-126. Pyro-Magneto Electric Devices.

\begin{tabular}{c|c|c|c|c|}
\hline No. & NaME. & DATE. & No. & NaMT. \\
\hline 434,586 & Edison, T. A & DATE. & \\
\hline
\end{tabular}

171. GENERATION.-40. REGULATORS.

\begin{tabular}{|c|c|c|}
\hline 431,618 & Polson, G. A & July 8,1890 \\
\hline 31,812 & Johnson, E. H & July 8,1890 \\
\hline 33,269 & Belt, P. P -- & July 29,1890 \\
\hline 4,951 & Raughtigan, M... & Aug. 26, 1890 \\
\hline 11,108 & Higham, $\mathrm{D}_{\ldots} \ldots$ (Reis.) & Aug. 26,1890 \\
\hline 135,332 & 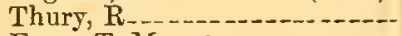 & Aug. 26, 1890 \\
\hline 35,490 & Foote, T. M _........... & Sept. 2,1890 \\
\hline 525 & Scribner, C. E_- & Sept. 2,1890 \\
\hline 6 & Scribner, C. E-_..... & Sept. 2, 1890 \\
\hline 35,527 & 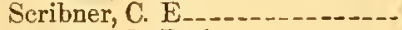 & Sept. 2, 1890 \\
\hline $35, \overline{0} 45$ & Prescott, G. B., jr & Sept. 2, 1890 \\
\hline 700 & Leonard, H. WV & Sept. 2,1890 \\
\hline 35,899 & 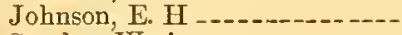 & Sept. 2, 1890 \\
\hline 5,982 & Stanley, W., jr $\ldots \ldots$ & Sept. 9,1890 \\
\hline 6,113 & Atwood, La M. C........ & Sept. 9,1890 \\
\hline 1 & 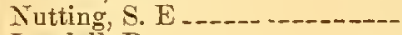 & Sept. 23,1890 \\
\hline 704 & Jundell, $\mathrm{R}$ & Oct. $\quad 7,1890$ \\
\hline 8,204 & Thomson, E & Oct. 14,1890 \\
\hline 656 & Thomson, E & Oct. 21,1890 \\
\hline 11 & Morrison, Wr & Oct. 28,1890 \\
\hline 30,1 & Gartner, $\mathrm{A}$ & Nov. 4,1890 \\
\hline 0 & Cleaver, F. J. and Fassold, G & Nor. 4,189 \\
\hline
\end{tabular}

\begin{tabular}{|c|c|c|}
\hline 440,590 & Hardy, F. D. & Nov. 11,1890 \\
\hline 441,665 & Hyer, W. E. & Dec. 2,1890 \\
\hline 441,794 & Freeman, W. K & Dec. 2,1890 \\
\hline 441,807 & Pepper, D., jr- & Dec. 2,1890 \\
\hline 442,668 & Rice, E. W., jr & Dec. 16,1890 \\
\hline $44 t, 416$ & Anthony, W. A & Jan, 13,1891 \\
\hline 444,587 & Ball, R. E & Jan. 13,1891 \\
\hline 445,448 & Holmes, J. H. & Jan. 27, 1891 \\
\hline 446,229 & Harris, L. S & Feb. 10,1891 \\
\hline 446,284 & Ellis, A. L & Feb. 10,1891 \\
\hline 448,681 & Short, S. H & Mar. 24, 1891 \\
\hline 449,245 & Fisher, B. H. & Mar. 31, 1891 \\
\hline 449,288 & Lahmeyer, $\mathrm{W}$ & Mar. 31,1891 \\
\hline 450,641 & Stanley, W., & Apr. 21, 1891 \\
\hline 450,985 & WVilliams, J, A & Apr. 21, 1891 \\
\hline 451,312 & Tischendoerfer, F _..... & Apr. 28,1891 \\
\hline 453,318 & Wessel, F. A & June 2,1891 \\
\hline 453,319 & Wessel, F. & June 2,1891 \\
\hline 453,320 & Wessel, & June 2,1891 \\
\hline 454,475 & Hering, C & June 23,1891 \\
\hline 4,476 & Hering, C & June 23, 1891 \\
\hline & & June 30,1 \\
\hline
\end{tabular}

171. GENERATION.-119. RegClatons-AlteryatiNG.

431,216 Stanley, Wr, jr

431,217 Stanley, W, jr

431,218 Stanley, Wr., jr July 1,1890 July 1,1890

July $1,1590 \quad 431,235$ Shallenberger, O. B ......... July 1,1890 44,939 Lemp, H. and Schmidt, L. M .- Jan. 20, 1891

171. GENERATION.-97. Systems of Distribetion.

431,460 Gutmanı, I

401,460 Gutmanu, L____._._. July 1, 1890 434,16: Shallenberger, O. B. and Bvl- Jug. 12, 1890 lesbr, H. II

434,614 Walter, H. E

438,308 Edison, T. A.
A ug. 19, 1890 Oct. 14,1890
40,224 De Ferranti, S. Z

441,542

414,503

445,657

446,515
Enholm, O.A

Hopkinson, $\mathrm{J}$

Bottome, T. D

Gutmann, L
Nov. 11,1890

Nov. 25,1890 Jan. 13,1891 Feb. 3, 1891 Feb. 17, 1891

172. MOTITE POWER. -9. CAR BRAKES.

438,452 Tan Depoele, C. J

441,215 . Theless, $\mathrm{M}$

443,661 Hunter, R. II $\begin{array}{lll}\text { Oct. } 14,1890 & 440,843 & \text { Blanchard, G. W- } \\ \text { Yor. } 25,1890 & 451,514 & \text { Payne, J. If }\end{array}$

Dec. 30,1590
$45 ?, 041$
Payne, J. J

Apr. 7,1891 May 5, 1891 May 12, 1891

172. MOTIYE POWER.-15. Dextal IXstromexts.

436,804 Roberts, MI. J

439,238 Faught, L. A _............. Oct. 2S, 1890 453,260 Kells, C. E., jr

Apr. $\quad \bar{i}, 1891$

June 2,1891

172. MOTIYE POWER.--75. LOCONOTIOX.

\begin{tabular}{l|l|lll}
432,646 & Smith, M. H. Hul $22,1890 \quad 444,480$ & Bentley, E. M
\end{tabular}

432,670 Mansfield, $\mathrm{F}$

tit,

Jan. 13,1891

172. MOTTE POWER.-36. MOTORS.

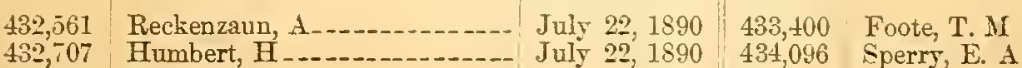

July 29,1890

432,988 Doyle, C. B

July 29,1890

434,180

Foote, T. M 
172. MOTIVE POWER.-36. Motors-Continued.

\begin{tabular}{|c|c|c|c|c|c|}
\hline So. & NAYIE. & DATE. & No. & NAME. & DATE. \\
\hline 39 & Blades, H. H & Sept. 2, 1890 & 448,603 & Chaplin, E. & Mar. 17, 1891 \\
\hline & Groswith, $\mathrm{H}$ - & Sept. 2, 1890 & 449,362 & Dean, J. H - & Mar. 31, 1891 \\
\hline & ightman, M. J & Sept. 9,1890 & 449,660 & Baxter, W., & Apr. 7,1891 \\
\hline & Mayer, M & Sept. 23,1890 & 450,441 & Whipple, F. F & Apr. 14,1891 \\
\hline & Rae, F. B & Sept. 30,1890 & 451,212 & Sharp, E. P & Apr. 28, 1891 \\
\hline & Emmner, J., jr--- & Oct. $\quad 7,1890$ & 451,559 & Hyer, W. E - & May 5,1891 \\
\hline+3 & Little, S. and S. J & Oet. 7,1890 & 451,870 & Hewett, E. M. G & May 5,1891 \\
\hline 837 & Buchanan, G & Oct. 21,1590 & ,S72 & La Boiteaux, F. M & May 5, 1891 \\
\hline 699 & Dressler, C. E-- & Nov. 18,1890 & 452,151 & Whittingham, G. H & May 12,1891 \\
\hline & Pullman, H. B. & Nov. 18,1890 & 452,214 & Chisholm, D. J & May 12,1891 \\
\hline 53 & , H & Dec. 2,1890 & $40-2,359$ & Briggs, G. T & May 19,1891 \\
\hline & $\mathrm{Gr}$ & Dec. 2,1 & 452 & Gra & May 19,1891 \\
\hline & , W. W & Dee. 9,1 & 795 & er, C. J & May 26,1891 \\
\hline 07 & H & Jan. 20, 1891 & $452,93 \pi$ & W. & May 26, 1891 \\
\hline 00 & , , M. J . & Jan. 20, 1891 & 453,032 & Blades, H. H & May 26,1891 \\
\hline & IV $\mathrm{X}$ & Jan. $2 \bar{i}, 1891$ & 45 & Macr & June 2,1891 \\
\hline & er, F. A_ & Feb. 3, 1891 & 453,367 & Haskins, C. C & June 2,1891 \\
\hline $\pm \pm 0.90 \%$ & E. P & Feb. 3,1891 & 454,024 & Blades, H. H & June 16,1891 \\
\hline & Blades, H. H & Mar. 3, 1891 & 454,626 & Rae, F. B & June 23,1891 \\
\hline & Traylo & Mar. 3, 1891 & 454,627 & $y, A . B$ & June 23,1891 \\
\hline 447,734 & $\begin{array}{l}\text { Mešrowitz, E. B. and Euchhop, } \\
\text { F. }\end{array}$ & Mar. 3, 1891 & $\begin{array}{l}454,852 \\
455,109\end{array}$ & $\begin{array}{l}\text { Scott, G. J } \\
\text { IIcGahan, F. L }\end{array}$ & $\begin{array}{l}\text { June } 30,1891 \\
\text { June } 30,1891\end{array}$ \\
\hline $\begin{array}{l}44,-27 \\
44,939\end{array}$ & $\begin{array}{l}\text { Hochhausen, TT } \\
\text { Chapman, IT. H }\end{array}$ & $\begin{array}{l}\text { Mar. 10, } 1891 \\
\text { Mar. } 10,1891\end{array}$ & 455,110 & MeGahan, F. L & June 30,1891 \\
\hline
\end{tabular}

172. MOTIVE POWER.-120. Motors-Alternating.

433,700

433,701

433,703

433,334

$43 \pm, 488$

435,603

439,042

439,459

439,102 443.950
Tesla, $\mathbf{N}$

Tesla, $\mathrm{N}$

The

Bradley, C. S _.......... Oct. 21, 1890

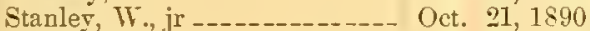

Zipernowsky, C., Déri, M. and Oct. 28, 1890 Blathy, O. T.

Bradley, C. S --

Tan Depoele, C. J.
Oct. 28,1890

Dec. 30,1890
444,188 Van Depoele, C. J

444,934 Billberg, C. O. C. and Winand, P. A. N

445,207 Tesla, N-

445,623 Patten, F. J

446,S64 Gutmann, L_-_._-_._._-_ Feb. 24, 1891

448,326 Eickemeyer, R_.._..._._. Mar. 17, 1891

450,541 Van Depoele, C. J._........ Apr. 14, 1891

455,067 Tesla, N.................... June 30, 1891
Jan. 6,1891 Jan. 20,1891 Jan. 27,1891

172. MOTIVE POWER-126. Motors-Reciprocating.

431,492 Van Depoele, C. J

431, 493 Tan Depoele, C. J

40,821

(1)

Bock, L., jr -.....

172. MOTIVE POIVER.-S. Steering ANd Propuliston.

$450,875 \quad$ Sims, IV. S

\begin{tabular}{|l|l|l|l} 
July 1,1890 & 450,543 & Van Depoele, C. J
\end{tabular}

July 1,1890 451,320 Eickemeyer, R.
Apr. 14, 1891

Apr. 28, 1891

(n)

172. MOTIVE POWER.-83. TraAsuission of PoWer.

\begin{tabular}{|c|c|c|c|c|c|}
\hline 431,170 & Marvin, H. N. & July 1,1890 & 436,275 & Van Depoele, C. & Sept. 9,1890 \\
\hline 431,494 & Van Depoele, C. J. & July 1,1890 & 436,276 & Van Depoele, C & Sept. 9,1890 \\
\hline 431,495 & Van Depoele, C. J- & July 1,1890 & 436,277 & Van Depoele, C. J & Sept. 9,1890 \\
\hline 431,496 & Tan Depoele, C. J & 1,1890 & 440,977 & Van Depoele, C. J & Nov. 18,1890 \\
\hline 433,758 & Zipernowsky, C. and Déri, M -. & Aug. 5,1890 & 444,265 & Bentley, E. M & Jan. 6,1891 \\
\hline 434,489 & Thomson, E & Aug. 19,1890 & 446,030 & Sperry, E. A & Feb. 10,1891 \\
\hline 434,816 & Van Depoele, C. J.-- & Aug. 19,1890 & 450,541 & Van Depoele, C. J & Apr. 14,1891 \\
\hline 435,261 & Tan Depoele, C. J & Aug. 26,1890 & 451,786 & Van Depoele, C. J & May 5,1891 \\
\hline $\begin{array}{l}435,983 \\
436,127\end{array}$ & $\begin{array}{l}\text { Stephen, W } \\
\text { Edison, T. A }\end{array}$ & $\begin{array}{l}\text { Sept. } 9,1890 \\
\text { Sept. } 9,1890\end{array}$ & 452,296 & Van Depoele, C. & May 12,1891 \\
\hline
\end{tabular}

173. CONDUCTORS.-13. CONDUCTORS.

432,310 Higham, D _._................ July 15, 1890 439, , TO6 Widdifield, W.P. and Bowman, Aug. 5, 1890 A. H.
433,917 493,920

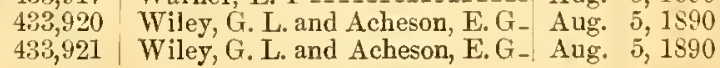


173. CONDUCTORS.-13. CONDUCTORS-Continued.

\begin{tabular}{|c|c|c|c|c|c|}
\hline No. & Name. & DATE. & No. & NAME. & Date. \\
\hline $\begin{array}{l}433,922 \\
434,885 \\
435,629 \\
436,432 \\
437,568 \\
437,632 \\
437,913 \\
438,003 \\
438,592 \\
441,837 \\
444,477 \\
446,655 \\
447,896 \\
447,897\end{array}$ & $\begin{array}{l}\text { Wiley, G. L. and Acheson, E. G- } \\
\text { Phillips, W. A- } \\
\text { Holcombe, A. G } \\
\text { Oehrle, W. E } \\
\text { Clark, E.-. } \\
\text { Splitdorf, C. F } \\
\text { Brooks, D., jr } \\
\text { Kundson, A. A } \\
\text { Eckert, W. H. and Gregory, } \\
\text { W. H. } \\
\text { Greenfield, E. T- } \\
\text { Wright, A. P } \\
\text { Arld, H. } \\
\text { Nash, F. J. } \\
\text { Nash, F. J. }\end{array}$ & $\begin{array}{l}\text { Aug. } 5,1890 \\
\text { Aug. 19, } 1890 \\
\text { Sept. 2, } 1890 \\
\text { Sept. 16, } 1890 \\
\text { Sept. } 30,1890 \\
\text { Sept. } 30,1890 \\
\text { Oct. } 7,1890 \\
\text { Oct. } 7,1890 \\
\text { Oct. } 14,1890 \\
\text { Dec. } 2,1890 \\
\text { Jan. } 13,1891 \\
\text { Feb. 17, } 1891 \\
\text { Mar. 10, } 1891 \\
\text { Mar. } 10,1891\end{array}$ & $\begin{array}{l}448,359 \\
448,604 \\
449,168 \\
449,400 \\
450,589 \\
450,734 \\
451,586 \\
451,587 \\
451,605 \\
452,091 \\
452,510 \\
452,565 \\
452,725 \\
454,060 \\
451,546\end{array}$ & $\begin{array}{l}\text { Nichols, H. B } \\
\text { Conner, W. A } \\
\text { Norman, T. W W } \\
\text { Bishop, J. D. } \\
\text { Marsh, J. IV } \\
\text { Bunker, W. I } \\
\text { Williams, J. B } \\
\text { Williams, J. B } \\
\text { Andrews, J. D. F } \\
\text { Williams, J. B } \\
\text { Williams, J. B } \\
\text { Curry, W. } \\
\text { Williams, J. B } \\
\text { McCracken, E. D } \\
\text { Sperry, A. W }\end{array}$ & $\begin{array}{l}\text { Mar. 17, } 1891 \\
\text { Mar. 17, } 1891 \\
\text { Mar. 31, 1891 } \\
\text { Mar. 31, 1891 } \\
\text { Apr. 14, 1891 } \\
\text { Apr. 21, 1891 } \\
\text { May 5, 1891 } \\
\text { May 5, } 1891 \\
\text { May 5, 1891 } \\
\text { May 12, 1891 } \\
\text { May 19, } 1891 \\
\text { May 19, } 1891 \\
\text { May 19, } 1891 \\
\text { June 16, 1891 } \\
\text { June 23, } 1891\end{array}$ \\
\hline
\end{tabular}

173. CONDUCTORS.-94. LEAD COVERED.

437,330 Brooks, D, jr 450,839 Walcott, J. E 452,340
Sept. 30, 1890 A pr. 21, 1891
452,341 Conner, IW. A

452,342 Conner, W. A
May 12, 1891 May 12, 1891

173. CONDUCTORS.-70. CONDUCTORS-UNDEREROUND.

431,780 Parsley, W. L

433,025 Patterson, W. R

434,076

434,165

437,126

437,445 439,391

441,043

441,810

442,162

442,868

443,523

Du $\mathrm{s}$,
July 8, 1890 July 29, 1890 Aug. 12,1890 Aug. 12, 1890 Sept. 23,1890 Sept. 30, 1890 Oct. 28. 1890 Nov. 18,1890 Dec. ¿, 1890 Dec. 9,1890 Dec. 16,1890 Dec. 30,1890

\section{3,524 \\ Cope, T. J-} 443,992

441,377

446,214

47,350

450,200

451,614

453,733

454,155

$45+, 275$

454,938

Cope, 'T. J.

454,939
Hart, IT. H

Witter, G--

Reilly, J. C

IVessel, F. A

Colbb, II. B

Price, J. E

Readman, IV

Johnson, E. H

Chenoweth, A.C

Chenoweth, A. C .......-..
Dec. 30,1890 Jan. 6, 1891 Jan. 6, 1891 Feb. 10, 1891 Mar. 3,1891 Apr. 14, 1891 May 5,1891 June 9,1891 June 16, 1891 June 16, 1891 June 30,1891 June 30, 1891

173. CONDUCTORS.-14. CONYECTORS

431,412 $\pm 33,948$ 434,085 434,892 434,943 436,857 437,116 438,400 441,919 442,370 445,365 445,751 446,871
Studte, H

Lieb, C. A

Lieb, C. A

Scott, G. J

Orcutt, E. I

Jones, F. F

Hinpher, J. J

Brennen, G. J

Banta, W. E

Garland, W. H

Bergmann, S

Holfman, J. IT

Short, S. H

Lieb, C. A

July 1,1890
Aug. 12,1890
Aug. 12,1890
Aug. 19, 1890
Aug. 26,1890
Sept. 23,1890
Scpt. 23,1890
Oct. 14,1890
Dec. 2,1890
Dec. 9, 1890
Jan. 27,1891
Feb. 3,1891
Feb. 3,1891
Feb. 24, 1891

447,833

$+48,680$

449,307

449,721

451,364

451,933

15.

453,225

$45+181$

454,430

454,669

454,794
Kuight, C. M. and Hawken, T - Mar. 10, 1891 Shlaudeman, F__._._._._._. Mar. 2t, 1891 Ball, 1H. P_________._._. Mar. 31, 1891 Wheeler, G. K_._._._._._._. Apr. 7, 1891 Brewer, H. J _._......... A pr. 28, 1891 Hunt, A. M _..._._._._._. May 12, 1891 Carroll, E. and Lambert, T. B.- May 19, 1891 W'heeler, F _................ June 2, 1891 Nash, E. L., Stout, G. G. and June 16, 1891 Davis, J. R.

Greenfield, E. T_._-______... June 16, 1891 Strauss, J. B _..._-_._._-_. June 23, 1891

Bickford, J. H _..._._........ June 23, 1891

173. CONDUCTORS.-28. Ixsulators

Furloug, L

Dunbar, J. K

Oakman. $\mathrm{S}$

IInehlbcrg, C. O

Chubbuck, H. E

Gaynor, T.

Short, S. H

Lessler,

Bain, F ...........

Potter,J. S. and Cartwright, D.J
Aug. 5,1890 Aug. 12, 1890 Aug. 19, 1890 Aug. 26, 1890 Sept. 9, 1890 Oct. $\quad$ T, 1890 Oct. 14,1890 Nov. 4,1890 Dec. 2, 1890

Dec. 23,1890 Jan. 6, 1891
414,879 445,790 445.969 448,395 448,95 450,384 450,708 451,379 451,950 453,036
Elkins, C Winton, $\mathrm{H}$. D

Branch, J. R

Feb. 10, 1891

YThceler, G. K _..........._._. Mar. 17, 1891 Graham, G. H. and Gaunane, T- Mar. 24, 1891 Bertolette, D. A-.......... Apr. 14, 1891 Creighton, T__.__________. Apr. 21, 1891 Haines, R. D .............. Apr. 28, 1891

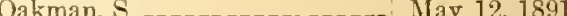
Brady, T. H 
173. CONDUCTORS.-31. LightNiNg Rods.

\begin{tabular}{|c|c|c|c|c|c|}
\hline No. & NAME. & DATE. & No. & Name. & Date. \\
\hline $\begin{array}{l}433,459 \\
437,526 \\
442,048\end{array}$ & $\begin{array}{l}\text { Ferrell, I. MI } \\
\text { Spang, H. WT } \\
\text { Hodges, X. D. C }\end{array}$ & $\begin{array}{l}\text { Aug. } 5,1890 \\
\text { Sept. } 30,1890 \\
\text { Dec. } 16,1890\end{array}$ & $\begin{array}{l}446,130 \\
452,796 \\
452,983\end{array}$ & $\begin{array}{l}\text { Downey, G. W } \\
\text { Kress, G. R. } \\
\text { Radford, C. M }\end{array}$ & $\begin{array}{l}\text { Feb. } 10,1891 \\
\text { May 26, } 1891 \\
\text { May 26, } 1891\end{array}$ \\
\hline
\end{tabular}

173. CONDUCTORS. - -43. Rheostats.

\begin{tabular}{|c|c|c|c|c|c|}
\hline 432,131 & Flemming, $\mathrm{O}$ & July 15,1890 & 441,293 & Doyle, J & Nor. 25,1890 \\
\hline 279 & Massey, G. B $\ldots \ldots$ & July 15,1890 & 441,838 & Greenfield, I & Dec. 2,1890 \\
\hline 2,894 & $n+\ldots-n---n$ & July 22,1890 & 441,918 & Ames, T. K & Dee. 2, 1890 \\
\hline 0.03 & Wessel, F. A. & Aug. 19,1890 & 444,707 & Lehman, J. I & Jan. 13,1891 \\
\hline & Thone, $F$ & Sept. 9, 1890 & $446, \pm 90$ & Weston, E & Feb. 17,1891 \\
\hline & Bentley, E. MI & Sept.23, 1890 & 447,494 & O'Meara, J & Mar. 3, 1891 \\
\hline & aing, J. H & Sept. 23,1890 & 448,682 & Short, S. H & Mar. 24, 1891 \\
\hline & Deprez, $\mathrm{M}_{-}$ & Sept. 30, 1890 & 452,574 & Jenney, C. D .......... & May 19,1891 \\
\hline & Vetter, J. C....... & Oet. 7,1890 & 454,207 & Eiekemeyer, R., jr & June 16,1891 \\
\hline & Gilbert, E. R & Nov. 4, 1890 & 454,969 & Sigsbee, C. D., Hayward, T. S. & June 30,1891 \\
\hline & $-----\infty$ & Nov. 11,1590 & & on, & \\
\hline
\end{tabular}

174. MEDICAL AND SURGICAL.—87. BAtHs.

$443,-580$ Lrke, $\mathbf{N}$

Dec. 30,1890

174. MEDICAL AND SURGICAL.-88. BODY-WEAR.

431,978 Jaekson, A. W.

434,746 Williams, R. E

437,079 Ashbrook, L. D

439,347

443,226

441,599

444,735
Fields, J. F

Pratt, J. L

Orren, A

Thomas, M. E -
July $8,1890 \quad 447,857$ Aug. $19,1890 \quad 148,128$

Sept. 23, 1890 Oet. 28,1890 Dec. 23,1890

Jan. 13,1891

Jan. 13,1891
448,953 449,345 $\$ 52,250$

$4.54,313$

454,573
Williams, P. G

Crisp, J. A. and Webb, G. F.

Fuller, W. C

Andrus, D. P

Williams, C. D

Webb, G. F

Sherman, W. N -
Mar. 10, 1891 Mar. 10, 1891 Mar. 24, 1891 Mar. 31, 1891 May 12, 1891 June 16, 1891 June 23, 1891

174. MEDICAL AND SURGiCAL.-89. EleCtrodes.

433,384 Woodward, J. H

434,024

435,343

435,376

438.041

444,597

Watkins, R. I
Toodwath, J and Guly 29, 1890

Brown, F. H.

Liehtenstadt, D. and C ....... Jan. 13, 1891
444,881 Gardiner, J. B

445,636 Chambers, J. C

449,651 Partseh, E.

450,690 Wilson, T. S

452,220 Gunning, J. H

453,476 Harness, C. B
Jan. 20, 1891 Feb. 3, 1891 Mar. 31, 1891 Apr. 21, 1891 May 12, 1891 June 2, 1891

175. SPECTAL APPLICATIONS.-4. APPLICATIONS-GENERAL.

431,776 436,205 443,282

445,860
Miller, J. D. and Dofflemyre, J. A Berlin, C. F C. F.

\section{July 8,1890} Sept. 9, 1890 Dec. 23,1890 Feb. 3, 1891
446,703 Dewey, M. W

450,872 Reardon, C. B

451,603 Sterry, T. N

452,571 Holson, A. B

454,717
Holson, A. B
Feb. 17, 1891 Apr. 21, 1891 May 5,1891 May 19, 1891 June 28, 1891

175. SPECIAL APPLICATIONS.-5. APPliCations-Tools and Machines.

July 15,1890 July 15,1890 July 15,1890 Aug. 12, 1890 Aug. 19, 1890 Sept. 23, 1890 Oct. 28, 1890 Oet. 28,1890 Oct. 28, 1890 Dee. 9, 1890 Dec. 16,1890
443,283

443,646 443,772 444,640 449,611 449,661 449,662 454,095 $45+462$ 454,832
Holmes, H. E. and Grosvenor, C. F.

Kilbourn, W. H Kells, C. E., jr Baxter, W., jr_._..._._._. Nar. 31, 1891 Baxter, W., jr_.............. Apr. 7, 1891 Baxter, W. jr______. Apr. 7, 1891 Wakefield, W. O _............ June 16, 1891 Eickemeyer, $R_{\ldots} \ldots$ Bennett, E. S. and Parshall, H. F June 23, 1891 
175. SPECIAL APPLICATIONS.-21. Electro-Magnets.

\begin{tabular}{|c|c|c|c|c|c|}
\hline No. & Name. & Date. & No. & NAYYE. & Date. \\
\hline $\begin{array}{l}432,896 \\
438,780 \\
449,358\end{array}$ & $\begin{array}{l}\text { Porter, C. S } \\
\text { Schmid, F. M } \\
\text { Wessel, F. A }\end{array}$ & $\begin{array}{l}\text { July } 22,1890 \\
\text { Oct. } 21,1890 \\
\text { Mar. } 31,1891\end{array}$ & $\begin{array}{l}451,308 \\
452,003\end{array}$ & $\begin{array}{l}\text { Park, H. S } \\
\text { Lipe, C. E- }\end{array}$ & $\begin{array}{l}\text { Apr. 28, } 1891 \\
\text { Uay 12, } 1891\end{array}$ \\
\hline
\end{tabular}

175. SPECIAL APPLICATIONS.-16. HEATFRS.

\begin{tabular}{|c|c|c|c|c|c|}
\hline & offin, C. L - & July 1,1890 & 64 & Thomson, E & Nov. 18.18 \\
\hline & aughlin, $\mathrm{J}$. & July 15,1890 & & & Nov. 25,1890 \\
\hline & Lemp, H. and Tregoning, J & July 22,1890 & & age, C. $\mathrm{H}$ & Dec. 16,1890 \\
\hline & $\mathrm{H}_{-}$ & July 22, 1890 & & en, & Dec. 16,1890 \\
\hline & n, E -.. & July 22, 1890 & & en, & Dec. 16,1890 \\
\hline & $\mathrm{n}, \mathrm{E}$ - & July 22,1890 & & C. $\mathbf{L}$ & Dec. 16,1890 \\
\hline & $\mathrm{E}$ & July 22,1890 & & $\mathrm{H}$ & Dec. 23,1890 \\
\hline & I. W & July 22, 1890 & & M. & Dec. 30,1890 \\
\hline & . L. & July 22, 1890 & & 19 & Dec. 30 \\
\hline & , C. E & Aug. 5,1 & & & Jan. 2 \\
\hline & I. E - & Aug. 12, 1890 & & d Angell, E. & Jan. 2 \\
\hline & & 12 & & 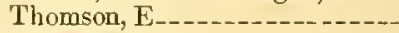 & Jan. 2 \\
\hline & & 19,1 & & & Jan. 2 \\
\hline & -.- & 19,1 & & $F$ & Jan. 2 \\
\hline & & 19 & & & Jan. 2 \\
\hline & E -.. & 19 & & i. IV & Feb. \\
\hline & E --- & 19 & & & Feb. \\
\hline & $\mathrm{D}$ & .26]$, & & & Feb. 1 \\
\hline & X. & $.26,1$ & & & Feb. 1 \\
\hline & & 26 & & idt, I & . 2 \\
\hline & $\mathrm{L}_{-1}$ & $.26,1$ & & , C. I & Feb. 2 \\
\hline & I. W- & t. 2 & & & Feb. 2 \\
\hline & I. W- & t. 2, & & & $\mathrm{Fe}$ \\
\hline & , C.E & Sept. 9, 1 & & & .2 \\
\hline & I & t. 9,1 & & $\mathrm{C}_{-}$ & . \\
\hline & $n$ & Sept. 16 & & & \\
\hline & $\mathrm{E}_{-}$ & t. 23 & & & \\
\hline & & 30 & & & \\
\hline & & 0 & & & \\
\hline & 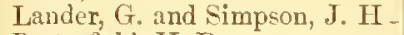 & $.30-3$ & & & \\
\hline & 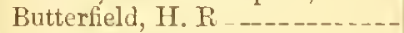 & -7 & & & \\
\hline & & Oct. 14, & & & \\
\hline & & 1 & & & \\
\hline & 1 & Oct. 14, & & I. $W=$ & \\
\hline & & 1 & & & \\
\hline & & t. 1 . & & & 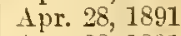 \\
\hline & & Oct. 21,1 & & $\mathrm{D}$ & Apr. 2 \\
\hline & & t. 21,1 & & & \\
\hline & $D$ & Oet. 21, & & $\mathrm{R}$ & May 12,1891 \\
\hline & Francis, E. & Nor. 4, & & & May 1 \\
\hline & & $\therefore 7$ & & & \\
\hline & nd J. W-- & Nov. 11,1 & & AI & June 23, \\
\hline & & & & Francis, E. & \\
\hline & & & & & Jur \\
\hline
\end{tabular}

175. SPECIAL APPLICATIONS-115. I+:ITLXG DEVICES-AUTOMA'TIC.

441,458 White, L. R. S _._._._..... Nov, 25, $1890 \quad 445,727$ Cutler, H. H

444,706 Lane, T. W.

Feb. 3, 1891 Feb. 3, 1891

44,896 , Lane, T. IY

175. SPECIAL APPLICATIONS.-116. ImNITIAG DEVICES-MaNUAL.

432,197 Graham, $\mathrm{R}$

432,215 Redding, H

$432,88 \pm$
July 15, 1890 , 436,354 Hayden, M. MI July 15,1890 453,618 Brown, H. B July 22, 1890
Sept. 16, 1890 June 9,1891

175. SPECIAL APPLICATIONS-25. IxCRUSTATIOX.

435,518 Pollok, J-488,579
Faunce, A. B. and Cabell, S. G
Sept. 2, 1890

Oct. $1 \pm, 1890$
443,598 Langstaffe, J-

Dec. 30,1890 
175. SPECIAL APPLICATIONS.-93. MagNetic Locks AND Bolts.

\begin{tabular}{|c|c|c|c|c|c|}
\hline No. & NAME. & DATE. & No. & NAME. & Date. \\
\hline $\begin{array}{l}437,692 \\
438,236 \\
416,509 \\
446,967\end{array}$ & $\begin{array}{l}\text { Damon, G. L } \\
\text { Hollar, IT. H. and Holmes, F.S } \\
\text { Colby, E. J } \\
\text { Flowers, I. I. }\end{array}$ & $\begin{array}{l}\text { Oct. } 7,1890 \\
\text { Oct. } 14,1890 \\
\text { Feb. } 17,1891 \\
\text { Feb. } 24,1891\end{array}$ & $\begin{array}{l}447,409 \\
448,110 \\
450,068\end{array}$ & $\begin{array}{l}\text { Kaiser, J. H. and Ledwith, A. B } \\
\text { Bates, L. } \\
\text { Grefen, H }\end{array}$ & $\begin{array}{l}\text { Mar. } 3,1891 \\
\text { Mar. 10, } 1891 \\
\text { Apr. } 7,1891\end{array}$ \\
\hline
\end{tabular}

175. SPECLAL APPLICATIONS.-90. SEPARATORS.

\begin{tabular}{|c|c|c|c|c|c|}
\hline $43:, 823$ & Finner, G. S-_ & July 22, 1890 & 444,223 & Payne, C. Q & Jan. 6,1891 \\
\hline se & Edison, T. A. and Dickson, W. & Aug. 19,1890 & $\begin{array}{l}446,70 \pm \\
446,767\end{array}$ & Edwards, W. C & Feb. 17, 1891 \\
\hline & MeMahan, J. M_. & Oct. 14,1890 & 449,610 & Moffatt, R. R . & Mar. 31, 1891 \\
\hline & Richards, F. H & Oct. 21,1890 & 449,726 & Ball, C. M. and Norton, S & A pr. 7,1891 \\
\hline & Von Syo, E-. & Nov. 4,1890 & 451,208 & Edwards, W. C & Apr. 28, 1891 \\
\hline & Conkling, $\mathrm{G}$. & Nov. 18,1890 & 451,369 & Conkling, J. P & Apr. 28,1 \\
\hline & Finney, G. S- & Dec. 16,1890 & 451,370 & Conkling, J. P & Apr. 28, 1891 \\
\hline & Finney, G. S. & Dec. 16,1890 & 453,317 & Townsend, H. C. & June 2,1891 \\
\hline & Fir & Dec. 16,1890 & $45 \pm, 555$ & Atkins, C. H & June 23,189 \\
\hline
\end{tabular}

175. SPECIAL APPLICATIONS.-84. VALve CoNTrollers.

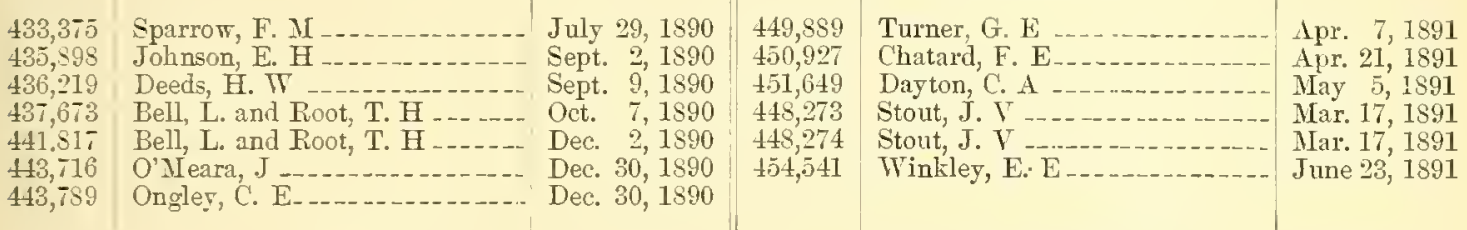

176. ELECTRIC LiGHTING.-80. CUt-OUtS-Electro-MAgNetic.

\begin{tabular}{|c|c|c|c|c|c|}
\hline 431,035 & Daft, L-- & July 1,1890 & 447,676 & Gammon, G. C- & Mar: 3, 1891 \\
\hline 78 & Chase, H. A . & July 29,1890 & 447,826 & Hochhausen, W & Mar. 10, 1891 \\
\hline & Chase, H. $A$. & July 29,1890 & 448,166 & Sweet, E. A. & Mar. 10,1891 \\
\hline 96 & Johnson, E. H. & Sept. 2, 1890 & 453,572 & Baumann, $\mathrm{R}$. & June 2, 1891 \\
\hline 30 & Edison, T. A & Oct. 14,1890 & $4 \overline{4}, 782$ & Thomson, E & June 23,1891 \\
\hline 19 & Mingle, G. W... & Dec. 23,1890 & 45 & Clausen, W. H & June 30, 1891 \\
\hline 902 & Tirrell, J. P & Feb. 24, 1891 & 455,092 & Billberg, C. O. C. and Winand, & June 30,1891 \\
\hline & laintor, & & & & \\
\hline
\end{tabular}

176. ELECTRIC LIGHTING.-78. CuT-OUTS-ThERuAL.

\begin{tabular}{|c|c|c|c|c|c|}
\hline & moda, J. L. anta & July & & F & V. 4 \\
\hline & a sor, L. B. & July & & $\operatorname{man}$, J. A & 11 \\
\hline & Favor, L. B-- & July 1,1890 & & , G. H. and Rice, E. W & Nov. 1s, 1890 \\
\hline & or, L. B. & July 8 , & & H. V & Nov. 18, \\
\hline & S. D. & July 22, & & right, D. & Dec. 2 \\
\hline & , H. A & July 29 , & & er, W. G & Dec. 30,1890 \\
\hline & $J$ & July $29^{\circ}$ & & W. G & Dee. 30 , \\
\hline & $\mathrm{E}$ & Iy 29, & & $T$ & n. 20 \\
\hline & UT & July 2 & & $\mathrm{E}_{-}$ & -0 \\
\hline & A - & July $29^{\circ}$ & & A & Jan. 27 \\
\hline & O & Aug. 5 , & & D & Feb. \\
\hline & , J. G. L $\ldots$ & Aug. $\bar{j}$, & & A. 1 & 24 \\
\hline & & g. 12 , & & & $\mathrm{Fel}$ \\
\hline & & & & M & \\
\hline & E. and Emmott, G & & & & \\
\hline & C. W & & & & \\
\hline & & & & & $\mathrm{M}$ \\
\hline & 5 & & & & \\
\hline & & & & & \\
\hline & $\mathrm{m}, \mathrm{G} \cdot \mathrm{H}$ & Se & & & \\
\hline & I & & & r. $\mathrm{H}$ & \\
\hline & E. E. and Cralbraith, J & Sept. 3 & & L. B & y 2 \\
\hline & 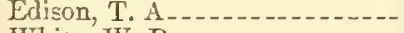 & Oct. & & E. $P$ & 91 \\
\hline & $-\cdots-\cdots--$ & & & & \\
\hline & $\mathrm{m}$ & $\begin{array}{l}\text { Oct. } \\
\text { Oct. }\end{array}$ & & & June \\
\hline & & & & & \\
\hline
\end{tabular}


176. ELECTRIC LIGHTING.-79. CUT-OUTS-MeChaNICAL.

\begin{tabular}{|c|c|c|c|c|c|}
\hline No. & NAMIE. & DATE. & No. & NAME. & D.tTE. \\
\hline
\end{tabular}

431,118

431,516

$432,-207$

432,234

433,359

433,883

434,151

434,152

434,899

435,093

435,098

435,132

435,152

$435,42 \pm$

436,050

436,087

436,107

436,122

437,352

437,359

437,513

437,607

438,118

438,167

438,934

439,602

440,308

440,614

140,720

440,545

441,588

442,030

442,585
Loomis, O. P

Tain Juy 1, 1890

McLaughlin, J. F ….... July 15, 1890

Frey, A. C_... July 15, 1890

McLaughlin, J. F _.......... July 29, 1890

Bergmann, S_............... Aug. 5, 1890

Lange, P. and Shallenberoer, O.B Aug. 12, 1890

Lange, $\Gamma$. and Shallenberger, O.B Aug. 12, 1890

Wilson, C. E__._........ Aug. 19, 1890

Herrick, C. II _. ..._._._._.. Aug. 26, 1890

Johnston, A. L_............ Aug. 26, 1890

Battershall, J. W..._...... Aug. 26, 1890

Norton, J. A............. Aug. 26, 1890

Huntington, C. W _........ Sept. 2, 1890

Gibbs, J. S. and Perkins, C. G-- Sept. 9, 1890

Perkins, C. G

Perkins, C.G

Battershall, J. IV

Hochliausen, IT

Pate, F. P

Stanley, L. T

Smith, II. F

Iarcher, T

Iiro, $\mathrm{F}$

McGregor, J.A. K

Bush, A. R

sept. 9,1890

Sept. 9,1890

Sept. 9, 1890

Sept. 30,1890

Sept. 30,1890

Sept. 30,1890

Sejt. 30,1890

Oct. 7,1890

Oct. $1 \pm, 1590$

Oet. 21,1890

Oct. 28,1890

Nor. 11,1890

Barberie, E.T. and Des Brisay, J Nov. 18, 1890

Pfluger, C. A

Herrick, C. II__._......... Nov. 18, 1890

Schultz, G............... Nor. 25, 1890

Fisher; F. E_-_______._. Dee. 2, 1890

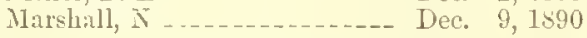

142,800 Colby, E. A

443,693 Wood, J.J J

444,374 IVilson, C. E

445,146 Keyes, B. B_..._........ Jan. 20, 1891

445,310 Cutter, G. and Armstrong, C. G- Jan. 27, 1891

445,362 Bergmann, S_.............. Jan. 27, 1891

445,752 Hoffman, J. IV ........... Feb. 3, 1891

445,957 Lean, G. R_......... Feb. 3, 1891

446,031 -Sperry, E. A _._........ Feb. 10, 1891

446,623 Lean, G. R_.............. 17, 1891

447,728 Hart, G. W _ Mar. 3, 1891

4\$7,788 Orford, J. M. and English, J. C. Mar. 10, 1891

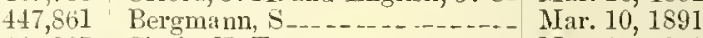

447,867 Clark, II. T__._._._._. Nar. 10, 1891

148,338 Hand, G. D. and Sells, O. P _- Nar. 17, 1891

448,431 Johnson, E. H _............ NIar. 17, 1891

448,698 Turner, D. B _............ NIar. 24, 1891

449,188 White, W

44,,282 Clark, H. T..... Mar. 31, 1891

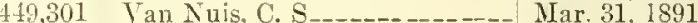

44, 3t8 Depp, O. J _._._._._._. Mar. 31, 1891

450,551 Adan, F. and Knapik, J _... Apr. 14, 1891

450,973 Norton, J. A__._.......... Apr. 21, 1891

45],190 White, L. P. S........ Apr. 28, 1891

451,267 Clark, H. T _............. Apr. 28, 1891

451,268 Clark, H. T_........... A pr. 28, 1891

452,173 Andrews, J. D. F.......... May 12, 1891

$\$ 53,638$ Furlong, I. F. and Tucker, F. O June 9, 1891

453,885 Mitchell, W _..._.......... June 9, 1891

$45 \pm, 087$ Teague, F _............... June 16, 1891

451,995 Des Brisay, J

176. ELECTRIC LIGHTING_-154. CGT-OUTS-MECHANICAL-OsCLLATLG SNaP.

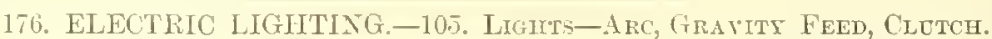

432,284

434,175

486,814

440,662

440,663

441,543

442,018

443,632

443,633

444,154

44,925

414,977
Russel1, E. C

Cady, WT. E-

Aug. 12, 1890

Thomson, F and Riee F. Sept. 23, 1890

Thomson, E. and Rice, E. W -- Nor. 18,1890

Enholm, O. A _.. Noy, 25, 1890

Crampton, T. P. Q ........ Dec. 2, 1890

Hejmann, E. and F. TI _..... Dece. 30, 1890

Hermann, E. and F. TT _....- I)ec. 30,1990

Skinner, J. J _............. Jan. 6, 1891

Thomson, E

Jan. 20,159$]$
445,136

445,724

$\pm 46,459$

446,668

$447,38$.

447,767

449,715

451,250

453.583

$45 \pm, 143$

$45,5,071$
Elkins, W. H

White, O.C

Mosher, J. A

Edison, T. A

Thomson, E

Folgason, H. C

Thonson, E. and Rice, E. W.,jr.

Waterhouse, A. G_.........

Hochhausen, IV

Me. Aley, J.E

Thompson, H. II
Jan. 20, 1891

Feb. 3, 1891

Feb. 17, 1891

Feb. 17, 1891

Mal. 3, 1891

Nar. 10, 1891

Apr. 7, 1891 Apr. 28, 1891 June 2,1891 June 16, 1891 June 30,1891

176. ELECTRIC LIGITTIN.-100. Lights-Are, Gravity Feed, Gear-Citum.

435,248 Rothlisberger, E

435,967

412,580

$4 \pm 2,617$

443,557

$444,10 \pm$

$4+4,472$

445,546

446,297

Graves, W. D

Henderson, W.

Giles, J. Ji

ITald $\mathrm{B}$

Easton, J. W

Schwarze, C. J
Whitcomb A Dec. 16, 1890
Aug. 26, $1890 \quad 448,41$

Sept. $9,1890 \quad 149,237$

449,235

151,101

451,189

451,309

453,693

Jan. 13,1801

Feb. 3, 1891

Feb. 10, 1891
Easton, J. IV

Ward, J. H. R.

Thard, J. H. R

Young, C. G

Ward, J. H. R.

Schefbauer, $\mathrm{R}$.

Ells, J. IV

454,485 Lemp, 足. and Wightman, M. J. June 23, 1891

176. ELECTRIC LiGhtiNG.107. Lights-Arc, Positrin Fen.

$436, \pm 65$ Tucker, C. A

437,767 Beach, R. H

410,604
Nutting, S. E
Sept. 16, 1890

Oct. 7, 1890

446,108

$\$ 16,109$

Noซ. 11, 1590
Mather, R. H.

Nather, R. II

Furmayer, $\mathrm{K}$
Feb. 10, 1891

Feb. 10, 1891

Mar. 17, 1891 
170. Electric Lighting.-108. Lights-Arc, Positive Feed, Clutch.

\begin{tabular}{|c|c|c|c|c|c|}
\hline No. & NAME. & DATE. & No. & NAME: & DATE. \\
\hline $\begin{array}{l}439,904 \\
45 \pm, 053\end{array}$ & $\begin{array}{l}\text { Shubert, H. C } \\
\text { Clarke, P }\end{array}$ & $\begin{array}{l}\text { Nov. 1, } 1890 \\
\text { June 16, } 1591\end{array}$ & 454,294 & Rushmore, S. WW & June 16,1891 \\
\hline
\end{tabular}

176. Electric LightiNG.-109. Lights-Arc, Positive Feed, Gear-Clutch.

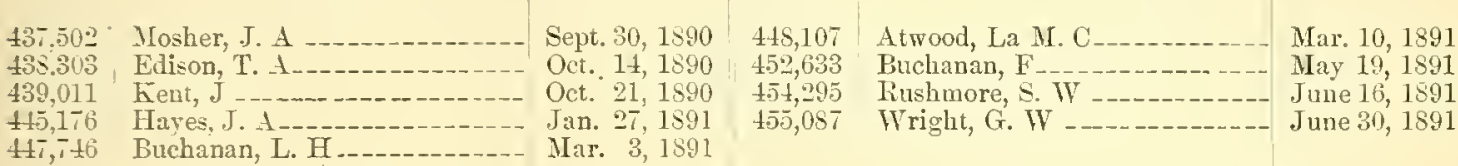

176. ELECTRIC LightiNG.-112. Lights-Aro, Special Features.

432.949 Lueas, A. H _._-_-_-_-_-_-_-- July 22, 1890

433,908 Parmly, S. P _............ Aug. 5, 1890

435,116 Gardiner, R. II _-_._-_._- Aug. 26,1890

437.739 Nichols, A. E.

437,901 Fazeltine, C. W-______- Oct. 7, 1890

412,127 Lonnsbury, J. A

413,914 Manning, J. A _-_._._._._. Dec. 30, 1890

444,330 Chase, J - Jan. 6, 1591
$444,473 \quad$ Ward, B. B

446,549 Russell, E. C

145,084 Seibold, A. C

449,153 Foster, H. A.

450,581

454,604

454,815
Gatley,

Sautter, $G$

Smith, G. W
Jan. 13,1891 Feb. 17, 1891 Mar. 10, 1891 IIar. 31, 1891 Mar. 31, 1891 Apr. 14, 1891 June 23, 1891 June 23, 1891

176. ELECTRIC LightixG.-102. Lights-Filanexts axd Carbons.

43:,710 Poland, L. S. P 433,041 Saunderson, L _........... July 29,1890 $43 \overline{5}, 660$ Hobby, V. XI _....... Sept. 2, 1890 435,134 Apps, 1._._._._._......... Oet. 14, 1890

438,299 Edison. T. A__._........... Oct. 14, 1890 439,393 Edison, T. A. \$10,759 Lemp, $\mathrm{H}_{-}$

414,471 Tard, B. B 415,371 Keen, T. M. B. and Haarmann, Jan. 27, 1891 WV. F.

446,660 Burns, ir. H

\section{6,669 Edison, T. A}

147,702

448,914

448,915

448,916

418,920

450,304

454,262

454,873

455,187

Seibold A. C

Erlwein, G Fr. Mar. 24, 1891 Erlwein, G _-_._._-_._._. Mar. 24, 1891 Heller, F. G. A Tan Choate, S. F _-____ Apr. 14, 1891 Edison, T. A_..._._._._._. June 16, 189 Lawrence, W. H _._........ June 30, 1891

176. ELECTRIC LIGHTING.-18. LIGHTS-INCANDESCENT.

431,776 434,159 433,298 439,175 411,127 441,128

441,530 445,688
Lean, G. R

Reinmann, A. L

Edison, T. A

Pauthonier, $\mathrm{C}=$

Bornholdt, A _....... Nov. 25, 1890

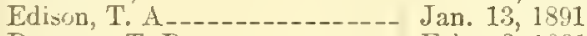

Bottome, T. D _............... Feb. 3, 1891
447,256 Nickerson, W. E. and Berrenberg, A.

45),494 Fessenden, R. A - May 19, 1891 452,780 Flagg, I. J._._._._._._........ May 26, 1891 453,742 Fessenden, R. A._.......... June 9, 1891 15. 45.5 S Edison, T, A $45 \overline{5}, 069$ Tesla, $\mathbb{N} \ldots \ldots$

176. Electrid Lightivg.-113. Lights-Incaxdesceirt, Special Features.

4:1,038 Dillon, L. W

$431,3,20$

431,051

$4: 31,721$

432,965

433,587

433,915

431149

434,917

431,925

49,863

435,665

436,677

$4.37,8.51$

$43 \times .301$

438,891

440,753

Pobb, J. 'T

Roberts, J.

Bathurst, F

\section{Bergmann, S-}

Jeob. S. J ..._............. July $S, 1890$

Aldrich, F. H__._._._._._. July 29, 1890

Little, L. IT. and Robb, J. T _- Aug. 5, 1890

Tregurtha, A. T__._._._. Aug. 5, 1890

Klein, C. J _._._._._._._._. Aug. 12, 1890

Chassagne, P. J ................. Aug. 26, 1890 Fitch, II. A._................. Aug. 26, 1890

Thorne, J. and Burr, E. B._. Sept. 16, 1890

Brown, J. E _._._.......... Oet. 7, 1890

Edison, T. A _.................. Oct. 14, 1890

Linn, S. H _................... 21, 1890
Nor. 18,1890
440,931
441,877
442,451
442,452
442,666
442,685
442,746
442,068
444,466
445,253
445,822
446,180
446,142
446,477
446,826
447,832

Tompkins, D. A

Lewis, $\mathrm{F}$

Priste, H. T

Paiste, H. T

Gunn, J

Moore, J. P

Bush, A. R

Cartwright, D. G. and Potter, Dec, 16, 1890

J.S.

Smith, T. C

I'otter, J. S

Jan. 27, 1891

Scott, G. A _ _ Feb. 3, 1891

Dergmann, S. and Klein, C. J.- Feb. 10, 1891

Martin G. IV _______ Feb. 10, 1891

Shillinglaw, A _..._....... Feb. 17, 1891

Wirt, H. C

Kint\&, J..._._._._......... Mir. 10, 1891 
176. Eleotric LightiNG.-113. Lights-Ixcandescent, Special Featdres-Continued.

\begin{tabular}{|c|c|c|c|c|c|}
\hline No. & NAME. & DATE. & No. & NAME. & DATE. \\
\hline $44 S, 164$ & Reinmann, C. F & Mar. 10,1891 & 451,149 & Bates, P. S & Apr. 28, 1891 \\
\hline 448,468 & Whitney, E. R & Mar. 17,1891 & 451,300 & Drew, H. P... & Apr. 28,1891 \\
\hline 449,266 & Lean, G. R $\ldots \ldots$ & Mar. 31, 1891 & 452,288 & Savage, W. W... & May 12,1891 \\
\hline 449,767 & Tregurtha, A. T_- & Apr. 7,1891 & 452,367 & Fischer, J. G _-- & May 19,1891 \\
\hline 449,921 & Tregurtha, A. T_..--_- & Apr. 7, 1891 & 453,041 & Drysdale, W. A & May 26, 1891 \\
\hline 450,000 & Carpenter, H. H........-- & Apr. 7,1891 & 454,184 & Pattison, R. and Desmond, D. G & June 16,1891 \\
\hline 450,991 & Cartwright, D. J $\ldots \cdots-\cdots$ & Apr. 21, 1891 & & & \\
\hline
\end{tabular}

176. ELECTRIC LIGHTING.-98. LIGHTS-SOCKETS AND KEYS.

\begin{tabular}{|c|c|c|c|c|c|}
\hline & & ly & & & Nov. 18, \\
\hline & chimer, $\mathbf{A}_{-}$ & July 29,1890 & t & ad Rich, A. E -.. & Dec. 9,1890 \\
\hline & Lebuck, T. G & Aug. 5,1890 & 04 & Cartwriglit, D.J & Dec. 23,1890 \\
\hline & , P. and Shallenberger, O. B & Aug. 12,1890 & & C. $A_{--}$ & Dec. 30,1890 \\
\hline & $\mathrm{ell}, \mathrm{F} \cdot \mathrm{C} \ldots$ & Aug. 26,1890 & & & Dec. 30,1890 \\
\hline & W. G - & 26,1890 & & 6 & Dec. 30,1894 \\
\hline & W. F. F & Aug. 26,1890 & & & Jan. 13,1891 \\
\hline & , W. F & Aug. 26,18 & & $\mathrm{a}, \mathrm{S}$ & Jan. 27,189 \\
\hline & $\mathrm{r}, \mathrm{C} \ldots \ldots-\cdots$ & 26,1 & & & Jan. 27,189$]$ \\
\hline & W. C - & Sept. 2, 1890 & & MI & Feb. 24,189 \\
\hline & , C. $\mathrm{II}_{--\ldots} \ldots$ & Sejit. 9,1 & & I. J & Mar. 3, 1891 \\
\hline & $\begin{array}{l}\text { J. S., Cartwright, D. J. } \\
\text { Keyes, B. B. }\end{array}$ & Sept. 9,1 & & & $\begin{array}{l}10 \\
17\end{array}$ \\
\hline & $\mathrm{E}$ & 16 & & & 24,1891 \\
\hline & F. $D^{\prime} A \ldots$ & Sept. 16,1 & & & 31 \\
\hline & , T. A $\mathrm{A}_{-\ldots}$ & Oct. $1 \pm, 1890$ & & TI & Apr. 21,189 \\
\hline & J. IV & Oct. 14,1890 & & $=---8$ & Apr. 21, 1891 \\
\hline & W. F. and Werline, F.H & Oct. 21,1 & & nd Wightman, M.J & May 5,1891 \\
\hline & J. $\mathrm{O} \ldots \ldots \ldots$ & Oct. 28,18 & & ley, C. H. and Porter, E. M & May 5, 189 \\
\hline & $-\cdots$ & Oct. 28,1890 & 45 & Keen, W. MI. B. and Haar- & May 12, \\
\hline & & Oct. & & & \\
\hline & & Oct. 28,18 & & & \\
\hline & - & Oct. 28,1890 & & $e$, & June 2 \\
\hline & 4 & Oct. 28,1890 & 45 & s, B. B & June 30 \\
\hline
\end{tabular}

176. ELECTBIC LIGHTING.-19. Srstems.

432,745

437,516

438,590

438,326

438,619

438,814

439,389

439,392

440,326

442,575

443,157

443,527

443,606

443,727

445,954

446,298

447,177
Cooke, E. 'T and Mackay, II'H July 22, 1890 Sawyer, R. F _._. Sept. 30, 1890 Woods, G. T.......... Oct. 14, 1s90 Ricliardson, G. W' $\mathrm{W}^{2} \ldots$ Oct. 14,1890 Giles, J. E................. 21, 1890 Kerstein, M _... Oet. 21, 1890

Edison, T. A _..... Oct. 28, 1890 Edison, T. 1..... Oct. 28, 1890 Richards, W. S _.......... Nor. 11, 1890

Eckert, IT. H. and Gregory, Dec. 9, 1890 IV. II.

Wyckoft, A. B

Jones, F. W -

Jolinson, E. H

Ongley, C. F

Jordau, L. F

Schwarze, C. J

Edwal'ds, T. MI
447,482 Ward, B. B

447,486

447,920

445,279

$449,0 \pm 4$

419,780

450,626

451,377

453,046

453,248

403,281

$45.3,349$

453,597

453,871

454,038

454,622

454,650 Tha, $\mathrm{N}-1$

Terstein, MI

Gould, R. H

ITall, G. L.

Smith, T. C

Young, C. G

Sawyer, R. F
Bennet, E. C. and Freeze, E. C-

Johnson, E. H

Yiiller, F, and Greene, J. G

La Roche, F. A

Storell, O. H

Fanning, J.

Tesla, N

Herzberg, I. and $\mathrm{A}$
Mar. 3, 1891

Ma1: 3, 1891 Mar. 10, 1891 Mar. 17, 1891 Mar. 24, 18.91 Apr. 7, 1891 Apr. 21, 1891 Apr. 28, 1891 May 26, 1891 June 2, 1891 June 2, 1891 June 2, 1891 June 2, 1891 June 9, 1891 June 16, 1891 June 23, 1891 June 23, 1891

176. ELECTRIC LIGHTING.-129. SrsteMS-CAR,

449,474 Gibbs, G -....-_..- Mar. 31, 1891 || 451,732 Jones, B. J _.

May 5,1891

176. ELECTRIC LIGHTING.-130. SYstems-SECONDARY BatTery. 
176. ELECTRIC LIGHTIXYG.-131. Sistens-Test.

\begin{tabular}{l|l|l|l|l}
\hline No. Name. & Date. & No. Name. & Date. \\
\hline
\end{tabular}

45ว.,วก3 Ebert, W. H

June 2, 1891

17. ELECTRIC SIGN ILING.-1. ALARNS.

\begin{tabular}{|c|c|c|c|c|}
\hline 431 & MacFarlane & July 1,1890 & 445,306 & A.E Jan. 27,1891 \\
\hline 431,885 & Focer, D & July 8,1890 & 445,495 & Brown, A. H \\
\hline 437,369 & Tschira, A-...-. & Sept. 30,1890 & 419,411 & Hannah, G \\
\hline $38, \overline{5} 98$ & Ashton, F. M & Oct. 21,1890 & $\$ 50,466$ & Ludlow, S. W \\
\hline
\end{tabular}

17\%. ELECTRIC SIGNALING. -2. ALARMS-BURGLdR.

\begin{tabular}{|c|c|c|c|c|c|}
\hline$+31,59$ & Lehr, G. B. & JulF 8,1890 & 410,015 & Pierce, F & Nor. 4.1890 \\
\hline 000 & Tilder, E. R _...-. & Sept. 9,1890 & 440,115 & Pierce, $F_{\ldots} \ldots$ & Noг. 4,1890 \\
\hline- & Ackermann, W. J _.. & Oct. $\quad 7,1890$ & 453,466 & Darling, E. A ....-- & June 2, 1891 \\
\hline $23 t$ & Gears, J _.......... & Oct. 14,1890 & $45 \overline{5}, 005$ & Palmer, D. A & 0,1891 \\
\hline
\end{tabular}

17\%. ELECTRIC SIGNALING.-3. ANNUNCIATORS.

\begin{tabular}{|c|c|c|c|c|c|}
\hline $433,58 \pm$ & Vining, C. J - & Aug. 5,1890 & 447,558 & Cram, W. E & Mar. 3, 1891 \\
\hline & Lnthe, J. W. and Jeavons, A. E & Sept. 2,1890 & 448,125 & Crabtree, L. T & Mar. 10,1891 \\
\hline & Ransom, G. F & 7,1890 & 448,169 & Warner, E. P . & Har. 10,1891 \\
\hline & Stanley, A. F - & Oet. 14,1890 & 449,690 & Newman, A & Apr. 7,1891 \\
\hline & Hale & Nov. 25,1890 & 450,390 & Varley, R., jr & Apr. 14,1891 \\
\hline & Holtzer, C. W_ & Nov. 25,1890 & 450,558 & Cox, î́ & Apr. 14,1891 \\
\hline & Fisher, F. E & Dec. 2,1890 & 450 & Delany, P. B. & Apr. 21, 1891 \\
\hline & Lathe, J. W. and Jearons, A. E & Dec. 30,1890 & 452,165 & Crane, M. G. and Cole, F. & May 12,1891 \\
\hline & Hormel, A & Jan. 6, 1891 & 453,416 & Pinolet, L. MI -- & June 2, 1891 \\
\hline & Cole, F. W & Feb. & 45 & $\mathrm{rn}, \mathrm{F} . \mathrm{W}$ & June 16,1891 \\
\hline & Gaynor, T. F & Feb. 10, 1 & 454,924 & Wood, F. B & June 30,1891 \\
\hline & Elfering, J. H & Feb. 17 & 455,016 & Harte, W. F. and Gerold, C. & June 30,1891 \\
\hline & Miller, & Feb. 24, 1891 & 455, & Wass, I & 1891 \\
\hline
\end{tabular}

17. ELECTRIC SIGNALING.-139. ANNEXCIATORS-DROP,

450,455 Gruschot, P. H.

451,226 , Ricketts, J. L
Apr. 14, 1891

A pr. 28,1891
452,585 Shirler, H. C

May 19,1891

17\%. ELECTRIC SIGNALING.-7. BELLS.

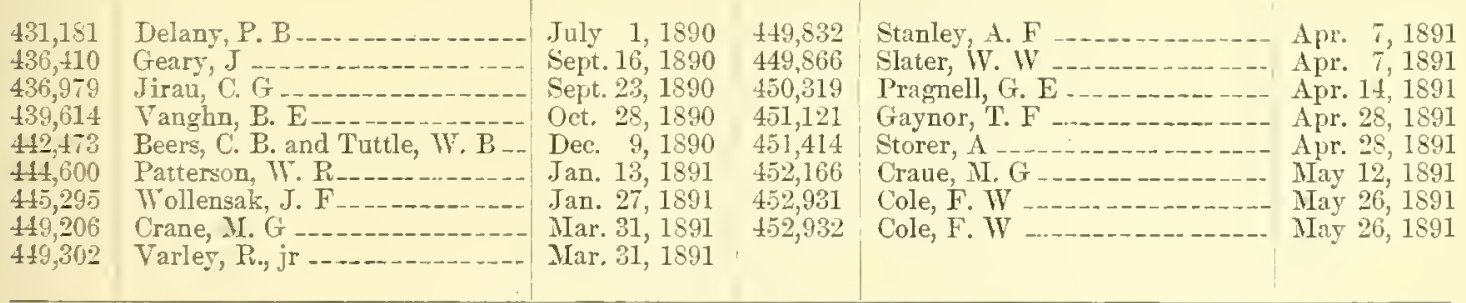

17. ELECTRIC SIGNALING.-26. INDICATORS.

432,694 Chappell, G. H

Glover, J. B

Compton, M. I

Goldstone, L

Conley, II

Casper, L.

Haight, H. J

Haight, H. J
Puobinson, W. E

Tanderhoef, G. $\mathrm{N}$
July 22,1890 July 29, 1890 July 29, 1890 Sept. 9, 1890 Nor. 4,1890 Nov. 11, 1890 Dec. 9, 1890 Dec. 16,1890 Dec. 16,1890 Dec. 16,1890
442,882 Haight, H. J

442,883 Haight, H. J

444,452

444,453

444,454

445,773

448,378

453,696

454,867
Mott, S. D

Nott, S. D

Mott, S. D

Ely, E. S

Soden, F. H

Hadlock, IV. I

Haight, H. J
Dec. 16,1890

Dec. 16,1890

Jan. 13, 1891 Jan. 13, 1891 Jan. 13, 1891 Feb. 3, 1891 Mlar. 17, 1891 June 9,1891 June 30, 1891 
177. ELECTRIC SIGNALING.-38. RALWAY SIGNALS.

\begin{tabular}{|c|c|c|}
\hline No. & ME. & DATE. \\
\hline 431,408 & Snee, IV. & July 1,1890 \\
\hline 431,671 & McCartney & July 8,1890 \\
\hline 433,959 & $\begin{array}{l}\text { Paul, IV. C. and Kleinstercher, } \\
\text { A. D. }\end{array}$ & Aug. 12,1890 \\
\hline 435,097 & Jacobs, S. J & Aug. 26, 1590 \\
\hline 435,105 & Stitzel, $\mathrm{F}$ & Ang. 26,1890 \\
\hline 435 & Thew, G - & Aug. 26,1890 \\
\hline 435,340 & Wilson, W. H...- & Aug. 26,1890 \\
\hline 435,482 & Conly, M. L & Sept. 2,1890 \\
\hline 16 & Perli, $T_{-}$ & Sept. 2,1890 \\
\hline 58 & Desant, II. F & Sept. 2,1890 \\
\hline 01 & Cortese, $\boldsymbol{M}_{-.}$ & Sept. 2,1890 \\
\hline 436,025 & Tower, G. A. & Sept. 9,1890 \\
\hline 154 & Schroen, F. C & Sept. 9,1890 \\
\hline 436,168 & Burt, E. M - & Sept. 9,1890 \\
\hline 371 & Morse, P. C. and Winton, H. D. & Sept. 16,1890 \\
\hline 436,777 & Davidson, B. R ........ & Sept. 28,1890 \\
\hline 348 & Powell, S. L & Sept. 30,1890 \\
\hline 084 & McElroy, D. S & Oct. 7,1890 \\
\hline 109 & Lattig, J. IV & Oct. 28,1890 \\
\hline 906 & Sprague, MY. B.and Kellogg, C. IV & Nor. 4, 1890 \\
\hline 41 & Ramsey, J., jr., and Weir, F. C. & Tov. 11,1890 \\
\hline 03 & Ramsey, J., jr., and Weir, F. C- & Nor. 11, 1590 \\
\hline 44 & Loom is, T. F & Јот. 18, 1890 \\
\hline & Riggs, J. IV - & Not. 18, 1890 \\
\hline & Riggs, J. W & సor. 18,1890 \\
\hline & Riggos, J. IV _- & Nor. 18,1890 \\
\hline 030 & 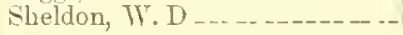 & For. 18,1890 \\
\hline
\end{tabular}

\begin{tabular}{|c|c|}
\hline No. & NAME. \\
\hline $441,0.31$ & Sheldon, IV. D - \\
\hline 411,033 & Sheldon, WV. D \\
\hline 411,034 & Sheldon, II. D $\ldots \ldots$ \\
\hline 441,041 & Waddell, W. H _............... \\
\hline 441,703 & Riggs, J. IV $\ldots$ \\
\hline 441,773 & Owen, R. O \\
\hline 442,339 & Schreuder, J. G _....-... \\
\hline $44: 074$ & Depp, O. J. and Munn, S. J \\
\hline $443,1.19$ & Powell, S. L \\
\hline 443,167 & Porell, S. L \\
\hline $443, \$ 14$ & Berue, A. IT \\
\hline 414,700 & 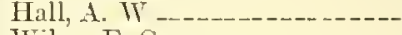 \\
\hline 445,106 & Triley, E. C $\ldots \ldots$ \\
\hline 445,564 & Loy, I. WT. and O'Toole, R .... \\
\hline 445,653 & Stitzel, F. and Weinedel, C--- \\
\hline 446,159 & $\begin{array}{l}\text { Westinghouse, G., jl., and } \\
\text { Schreuder, J. G. }\end{array}$ \\
\hline 446,313 & 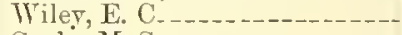 \\
\hline $\pm 46, \pm 03$ & Conly, M. S \\
\hline 446,546 & Cook, WV. S. and M. C. \\
\hline $\pm 16,917$ & Harres, E. T $\ldots \ldots$ \\
\hline 447,906 & Rosenfield, WT. TV \\
\hline 448,315 & Berne, A. WY \\
\hline 450,616 & Taylor, J. D \\
\hline 451,157 & 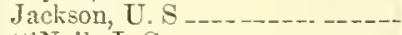 \\
\hline $45^{\circ}, 281$ & U'Teil, J. C - \\
\hline 454,245 & 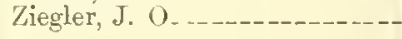 \\
\hline
\end{tabular}

DATE.

Nov. 1S, 1890

Nor. 18, 1890

Ког. 18, 1890

Nov. 18, 1890

Dec. 2, 1890

Dec. $\quad 2,1890$

Dec. 9, 1890

Dec. 16,1890

Dec. 23,1890

Dec. 23,1890

Dec. 30,1890

Jan. 13, 1591

Jan. 20, 1891

Feb. 3, 1891

Feb. 3, 1891

Feb. 10, 1891

Feb. 10, 1891

Feb. 10, 1891

Feb. 17, 1891

Feb. 24, 1891

Mar. 10, 1891

Mar. 17, 1891

Apr. 21, 1891

Apr. 28, 1891

Jlay 12, 1891

June 16, 1891

17\%. ELECTRIC SIGNALING-147. RAIITAY-BLOCE.

$-454,266$ Sterens, W. and Horey, H. J

June 30,1891

177. ELECTRIC SIGNALIYG.-146. RallwaY-BLOCK Srstents.

445,031 Harrison, E. L____._. Mar. 10, 1691 449,731 Carlton, J. A. and Johnston, Apr. 7, 1891 H. L.

177. ELECTRIC SIGXALING.-14!). RaILAT-CAß, Sectoxal RaIL.

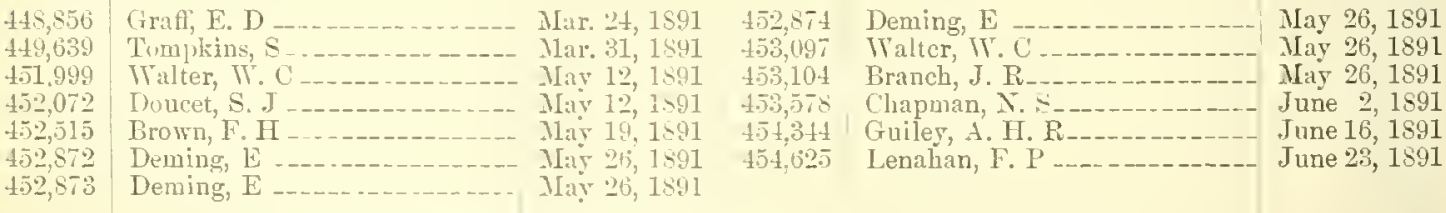

17. ELECTRIC SIGY.ALIYG-150. R.ILWAY-SECTIONAL RAIL.

\begin{tabular}{l|l}
448,751 & Kinsman, F. E \\
449,990 & Wilhelm, C. W
\end{tabular}

17. ELECTRIC SIG.XIING.-39. RECORDERS.

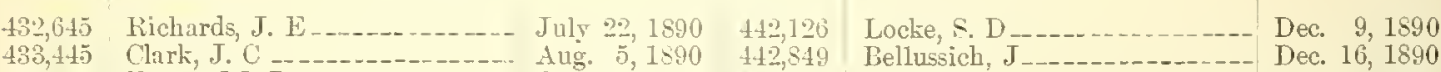

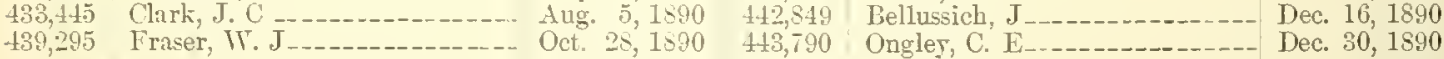

178. TELEGRAPHY.- 16 . Actomatic.

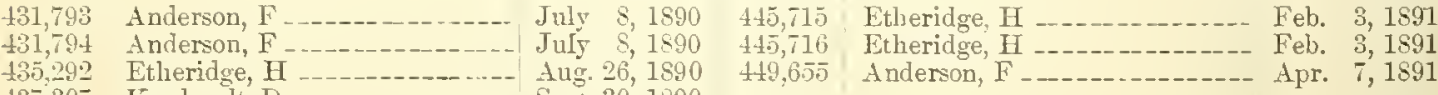

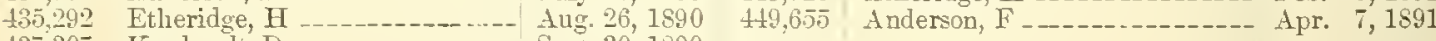

437,307 Kunhardt, D_-_._._....- Sept. 30,1890


178. TELEGRAPHY.-45. Circuits AxD Sistems.

\begin{tabular}{|c|c|c|c|c|c|}
\hline Yo. & NAME. & Date. & No. & Nane. & DAte. \\
\hline $\begin{array}{l}432,724 \\
433,082 \\
434,835 \\
435,679 \\
437,420\end{array}$ & $\begin{array}{l}\text { Carr, C. P } \\
\text { Jones, F. W } \\
\text { Pearson, P } \\
\text { Breed, G G } \\
\text { Edison, T. A- }\end{array}$ & $\begin{array}{l}\text { July } 22,1890 \\
\text { July } 29,1890 \\
\text { Aug. } 19,1890 \\
\text { Sept. } 2,1890 \\
\text { Sept. } 30,1890\end{array}$ & $\begin{array}{l}440,164 \\
440,199 \\
441,847 \\
442,808 \\
448,779\end{array}$ & $\begin{array}{l}\text { Keeley, D. H. } \\
\text { Roscbrugh, A. M } \\
\text { Jones, F. W } \\
\text { Hummcl, A. G. and Graham, F.A } \\
\text { Edison, T. A. }\end{array}$ & $\begin{array}{l}\text { Nor. } 11,1890 \\
\text { Nov. } 11,1890 \\
\text { Dec. 2, } 1890 \\
\text { Dec. 16, } 1890 \\
\text { Mar. 24, } 1891\end{array}$ \\
\hline
\end{tabular}

178. TELEGRAPHY.-47. DiA.

437,859 Kelley, D. and Parkhurst, M. C Oct. 7,1890

178. TELEGRAPHY.-48. DUPLEX.

\begin{tabular}{|c|c|c|c|c|c|}
\hline $\begin{array}{l}435,639 \\
435,851\end{array}$ & $\begin{array}{l}\text { Edison, T. A } \\
\text { Muirhead, A }\end{array}$ & $\begin{array}{ll}\text { Sept. } 2,1890 \\
\text { Sept. } 2,1890\end{array}$ & 452,913 & Edison, T. A & May 26,1891 \\
\hline
\end{tabular}

178. TELEGRAPHY.-19. Drvano.

$437,572 \mid$ Rae, F. B

Sept. 30,1890

178. TELEGRAPHY.--50. Fire.

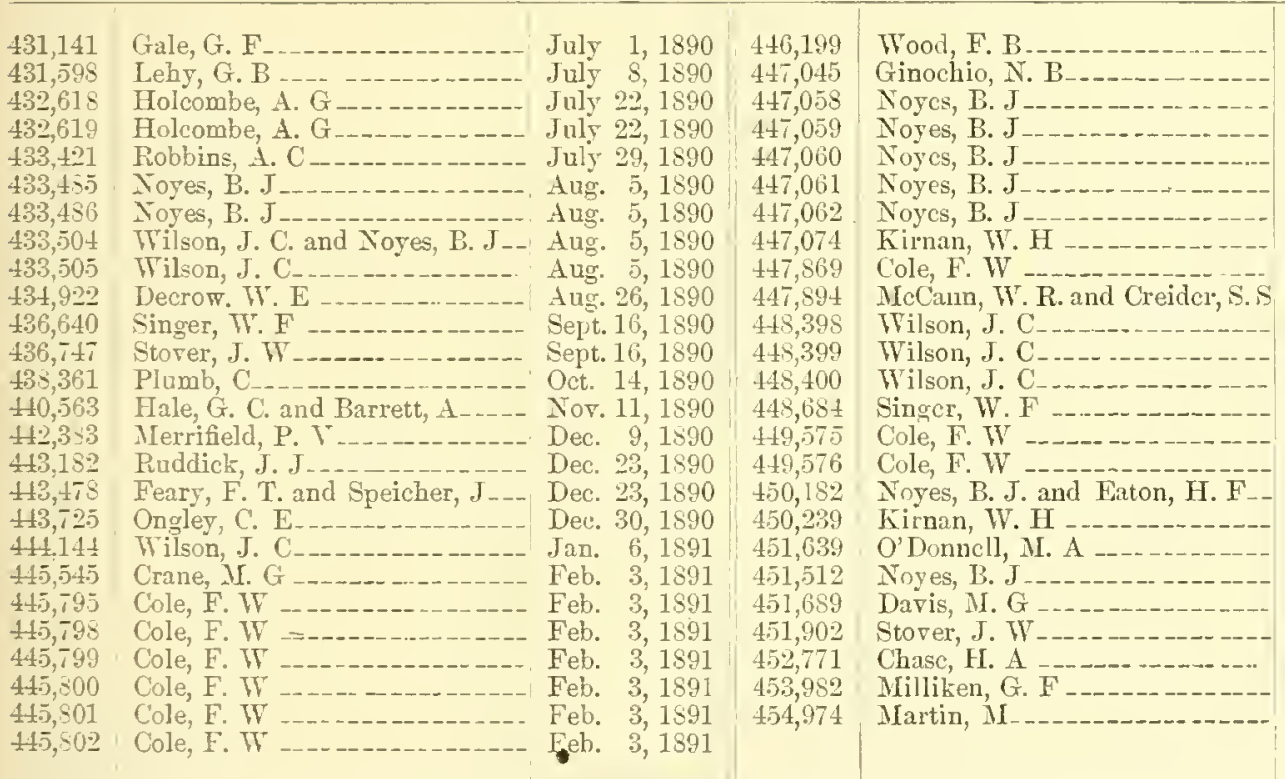

Feb. 10, 1891 Feb. 24, 1891 Feb. 24, 1591 Feb. 24, 1891 Feb. 24, 1891 Feb. 24, 1891 Feb. 24, 1891 Feb. 24, 1891 Mar. 10, 1891 Mar. 10, 1891 Mar. 17, 1891 Mar. 17, 1891 Mar. 17, 1891 Iar. 24, 1891 Mar. 31, 1891 Mar. 31, 1891 Apr. 14, 1891 Apr. 14, 1891 May 5, 1891 Nay 5, 1891 May 5,1891 May 5, 1891 May 26, 1891 June 9,1891 June 30,1891

178. TELEGRAPHY.-103. Fire, Alarms and Extinguishers.

431,979 Jacobs, H. E $\quad$ July 8, 1890 466,200 Broichoans, S

436,961 Upton, F. R. and Dibble, F. J - Sept. 23, $1890 \mid 447,998$ Adams, G. W

437,669 Young, J. Sept.30, 1890 449,829 Slocum, E. L

440,013 Chatfield, L. O -

442,385 Neu, G. S

443,724 Ongley, C. E.... Dec. 30, 1890

454,973

Martin, M

Feb. 10,1891 Nar. 10, 1891 Apr. 7, 1891 Apr. 14, 1891 May 19, 1891 June 30,1891

178. TELEGRAPHY.-160. Fre, Auromatic.

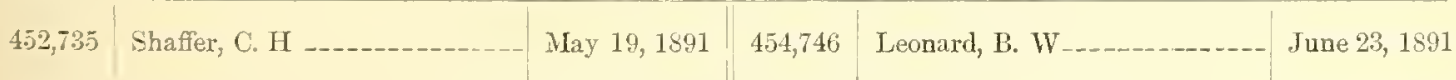

178. TELEGRAPHY.-163. Fire, NoN-INTERFering. 
178. TELEGRAPHY.-51. Multiplex.

\begin{tabular}{|c|c|c|c|c|c|}
\hline No. & NAMEE. & DATE. & No. & NAME. & DATE. \\
\hline 431,651 & 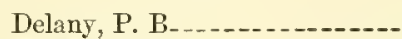 & July 8,1890 & 442,267 & Ires, E. B & Dec. 9,1890 \\
\hline 437,930 & Miner, W. M & Oet. 7,1890 & 419,897 & Brown, R. G & Apr. 7,1891 \\
\hline 440,165 & Keeley, D. H & Nov. 11,1890 & 446,424 & Brown, R. G. & Feb. 17,1891 \\
\hline 440,766 & Delany, P. B. & Nov. 18,1890 & 446,425 & Brown, R. G & Feb. 17,1891 \\
\hline 440,767 & Delany, P. B. & Nov. 18,1890 & 453,601 & Edison, T. A & June 2,1891 \\
\hline $4 \pm 0,-68$ & Delany, P. B & Nov. 18. 1890 & 454,630 & Toye, B. B & June 23,1891 \\
\hline
\end{tabular}

178. TELEgraphY.-54. Printing.

\begin{tabular}{|c|c|c|c|c|c|}
\hline 431,784 & $\operatorname{cott}$, G. B & July 8,1890 & 443,135 & Essick, S. V. B & Dec. 23,1890 \\
\hline & MeCoy, A. T & July 8,1890 & 443,136 & Essick, S. Y. B & Dec. 23,1890 \\
\hline & Taylor, W. W & July 15,1890 & 443,726 & Ongley, C. E- & Dec. 30,1890 \\
\hline & insky, A & July 15 & 450,228 & Fisk, P. H & Apr. 14,18 \\
\hline & T. M & Aug. 12, 1 & 450,630 & Linville, S. R. and Hettmans- & Apr. 21,18 \\
\hline & J.I & Aug. 19,18 & & S. R & Apr \\
\hline & J. I & Sept. 16, 1 & $4=$ & $\overline{\mathrm{P}}$ & Nay 1 \\
\hline & Taylor, W. W & Dec. 9 & 454,8 & Sedgwick, & June 3 \\
\hline & a & Dec. 28,1 & 455,294 & Bates, D. H. and Van Hoe & June 3 \\
\hline & ick, S. & Dec. 23 & & & \\
\hline
\end{tabular}

178. TELEgRaPH Y.-14:- Printivg, Wieel.

455,075 Van Hoevenlergh, $\mathrm{H}$

June 30,1891

17. TELEGRAPII,-56. RAIWAY-CAR.

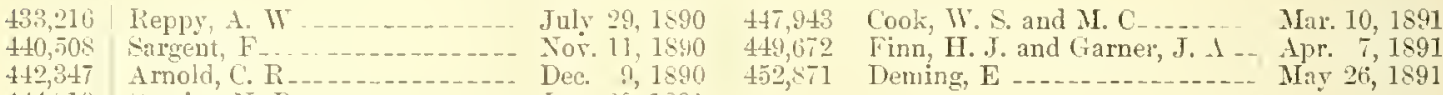

$44^{2} 347$ Amold C R - D 1890

44,619 Cregier, X. D

17S. TELEGRAPHY.-63. REFD.

439,017 Langdon-Davies, ( _ _..._._. Oct. 21, 1890 453,261 Langdon-Daries, C _._._._... June 2, 1891

447,198 Parker, J. $1 \ldots \ldots$ Feb. 24, 1891

178. TELEGRAPHY.-10. Circeit-CiJosers.

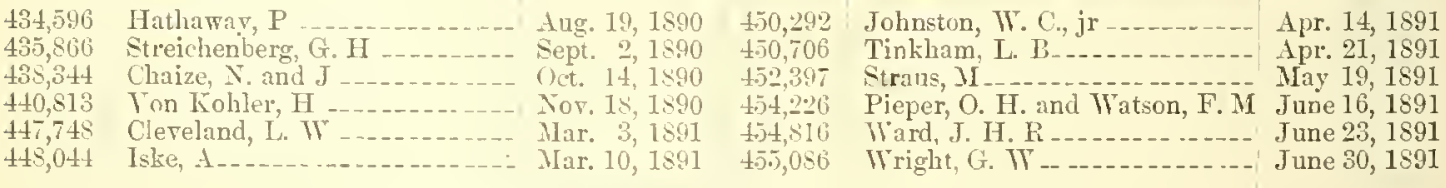

178. TELEGRAPIIY-12S. Cmocit-Cloners, Therial.

$439,4 \pm 1$ Summerskill, C. W

411,622

$4.5,931$

446,840

450,260
Shafter, C. H

Douse, T. R .

Barron, A. M

Singer, $\mathrm{W}$. $\mathrm{F}$
Oet. 28,1890 Tor. 25, 18,90 Feb. 3, 1891 Feb. 24, 1891 Apr. 14, 1891
450,425 450,590

451,548

$453,7=23$
Reinemann, $A$

Sims, J. C -

Zagelmeyer, I
Apr. 14, 1891 Apr. 21,1891 May 5,1891 June 9, 1891

178. TELEGRAPHY.-29. KEYs.

433,83̄ Yan Deusen, J. B 437,510 Purdy, A. F
Aug. 5,1890 Sept. 30,1890 +39,959
Oct. 14,1890 Nov. 4, 1890 
178. TELEGRAPHY.-30. LightNing Arresters.

\begin{tabular}{|c|c|c|c|c|c|}
\hline No. & NAME. & DATE. & No. & NAME. & DATE. \\
\hline 433,430 & Turts, A. and Baldwin, O. $\mathrm{H}_{--}$ & July 29,1890 & 440,654 & Rice, E. W., jr $\ldots$ & Nov. 18,1890 \\
\hline 433,682 & Jenks, W. J $\ldots$ & Aug. 5,1890 & 441,999 & Wood, J. J & Dec. 2,1890 \\
\hline 434,163 & Stilwell, L. B & Aug. 12. 1890 & 444,678 & 'Thomson, E - & Jan. 13,1891 \\
\hline 434,166 & WTurts, A & Aug. 1\%, 1890 & 449,903 & Easton, J. IV _-. & Apr. 7,1891 \\
\hline 434.167 & Wurts, A._. & Aug. 12, 1890 & 451,521 & Rice, E. W., jr..- & May 5, 1891 \\
\hline $43 \pm, 1 ; 0$ & Wurts, $\mathbf{A}_{--}$ & Aug. 12, 1890 & 454,496 & Rae, F. B & June 23,1891 \\
\hline 4334,371 & Tirts, $\mathrm{A}_{---}$ & Aug. 12,1890 & $\pm 54,67]$ & Thomson, E & June 23, 1891 \\
\hline 436,238 & Hoop, G. D-- & Sept. 9,1890 & 454,672 & Thomson, E & June 23,1891 \\
\hline 437,362 & Jenks, W. J. & Sept. 30,1890 & 4504,673 & "Thomson, F $\ldots$ & June 23,1891 \\
\hline 437,397 & Hudie, R.... & Sept. 30,1890 & 455,228 & Mansfield, F. and Wason, C. W- & June 30,1891 \\
\hline 438,788 & White, A. C. & Oct. 21,1890 & & & \\
\hline
\end{tabular}

178. TELEGRAPH Y.--41. Relars ANd Sounders.

432.098 Treber, J. M
434,585 Edison, T. A.

435,233

44,338
JuIy 15,1890

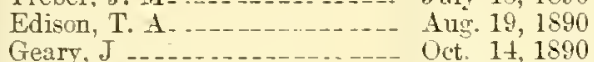
Sthreider, J. G Dec. 9,1890

\section{5,442 Doggett, J

450,272 Wickes, R. O \\ 454,338}

Jan. 27,1891 Apr. 14, 1891 June 16,1891

\section{TELEGRAPHY.-12. REPEATERS.}

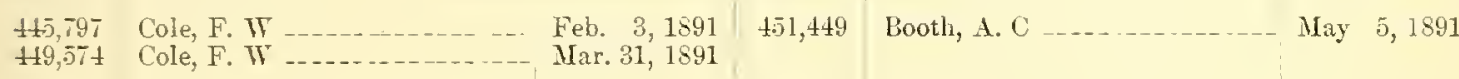

178. TELEGRAPHY.-69. TRANSMTTERS.

\begin{tabular}{|c|c|c|}
\hline 7 & Smith, S. WT & $\begin{array}{l}\text { Dec. } 23,1890 \\
\text { Feb. } 24,1891\end{array}$ \\
\hline & & \\
\hline
\end{tabular}

179. TELEPHONY. - 82. Axti-Induction Devices.

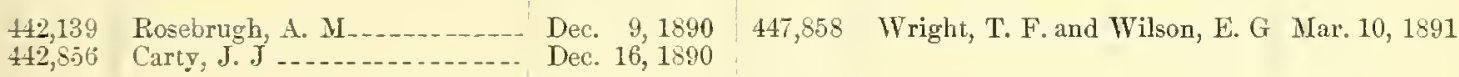

179. TELEPHONY.-81. Telephones-Conddctors, Awti-Inddetive.

\begin{tabular}{ll|l|l|l|l}
437,311 & MeClner, C. E & Sept. 30, 1890 & 454,890 & Thomson, E $\ldots \ldots$
\end{tabular}

179. TELEPHONY.-57. Telephones-CALLS.

\begin{tabular}{|c|c|c|c|c|}
\hline & $\begin{array}{l}\text { Vincent, C. H } \\
\text { Cutler, J. L } \\
\text { Hatch, A. A } \\
\text { Hatch, A. A } \\
\text { Edison, T. A. } \\
\text { McManman, J. A } \\
\text { Lawrence, G. R }\end{array}$ & $\begin{array}{l}\text { July } 1,1890 \\
\text { Aug. } 5,1890 \\
\text { Sept. 2, } 1890 \\
\text { Sept. 2, } 1890 \\
\text { Oct. 14, } 1890 \\
\text { Not. 18, } 1890 \\
\text { Jan. 13, } 1891\end{array}$ & & 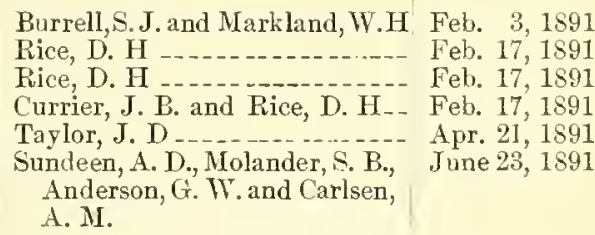 \\
\hline
\end{tabular}

179. TelephoNY.-143. Telephones-Calls, Step-BT-Step.

179. TELEPHONY.-5S. Telephones-Detams.

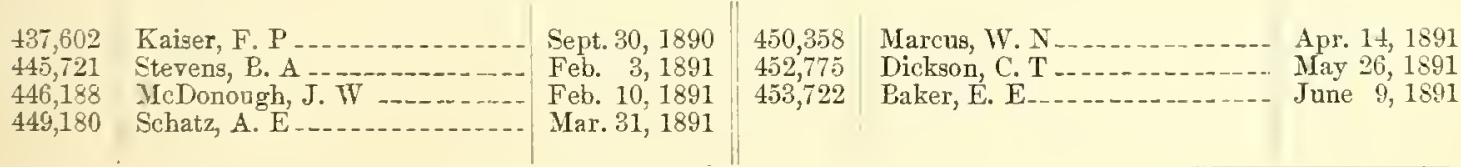


179. TELEPIIONY.-59. TELEPHONES-ELECTRIC.

\begin{tabular}{|c|c|c|c|c|c|}
\hline No. & NAME. & DATE. & No. & Name. & DATE. \\
\hline 433 & Field, S. D & July 29,1890 & 438,818 & McDonough, , & Oct. 21,1890 \\
\hline 433 & Dav & Aug. 12,1890 & 440,096 & Bergn & Nor. 4,1890 \\
\hline & d, T. D & Scpt. 2,1890 & 443,494 & Berg & Dec. 30,1890 \\
\hline 334 & Burr & Sept. 16,1890 & 443,526 & Johnson, E. H & Dec. 30,1890 \\
\hline 436,335 & Burn & Sept. 16, 1890 & 445,475 & Noriega, E .. & Jan. 27,1891 \\
\hline 436,513 & I, S. L _ . . & Sept. 16,1890 & 448,726 & Oeÿan, T_ & Mar. 24, 1891 \\
\hline 438,306 & T. A. and Gilliland, E. T & Oct. 14,1890 & 450,426 & Roulez, J. E- & Apr. 14,1891 \\
\hline 438,631 & MacDonald, A. J & Oct. 21,1890 & 452,636 & Fitzimmons, $\mathrm{P}$ & May 19,1891 \\
\hline 438,632 & MacDonald, A. J. & Oct. 21,1890 & $45 \cdot 2,758$ & Müller, A. C. F & May 19,1891 \\
\hline 438,784 & Stut, J. C. H & Oct. 21,1890 & 453,581 & Fitzimmons, $\mathrm{P}$ & June 2,1891 \\
\hline
\end{tabular}

179. TELEPHONY.-60. TELEPHONES-MAGNETIC.

436,512 Wiegand, S. L _._.... Sept. 16, 1890 447,194 Mlercadicr, E. J. P

438,828 Richards, W. L......... Oct. 21, 1890

441,396 Capps, F. L

443,493 Gergmaun, S__._._......... Dec. 30, 1890

\section{7,194 Mercadicr, E. J.P}

449,254 Humans, W

451,634 Corwiu, IV. S
Feb. 24, 1891

Mar. 17,1891 Nar, 31, 1891

May 5,1891

179. Telephony.-77. Telephones-Relays and Repeaters.

436,514 Wiegand, S. L

Sept. 16, $1890 \mid 448,153$ Ludwig, J. C

Mar. 10, 1891

179. TELEPHONY.-100. TELEPHONES-SUPPORTS.

431,810 Hess, E. C

432,883

436,717

437,927
July 8,1890

July 22,1590

Sept. 16, Is90

Mloore, J.T

II ughes, F.
440,091

$4,50,359$

453,435

454,138
Tinning, F. T. and W. K. S -.- Nov. 4, 1890 Nareus, II. N __._._._._. A pr. 14, 1891 Tinning, F. T. and W. K. S _... June 2, 1891

Mercadier, E. J.P _._._._-_._ June 16, 1891

179. TELEPHONY.-\$5. TELEPHONES SWITCH-BOARDS.

449,685 Lron, J. B

451,397 Chase, H. A

452,921 Lyon, J. B
Apr. 7,1891

Apr. 25, 1591

May 26, 1891
453,000 Goodridge, W. M

453,863 11ibbard, A.

454,584 Viles, F. T. and Young, $\mathrm{N}$
May 26, 1891 June 9,1891 June 23,1891

179. 'TELEPHONY.-64. TELEPHONES-SYSTEMS.

431,531 Sccly, J. A

431,962 Birton, E. M

432,547

433,046

433,047

433,424

433,636

437,010

437,012

437,986

438,982

440,1 Is

442,143

442,144

442,145

442,146
IeCoy, J. A

Scribner, C. F

Scribner, C. E

Seribner, C. F

Wainer, E. P

Bentley, E. II

Bentley, E. MI

Graham, $A$

Beach, F. G

Root, H. C

Scribner, C. F

Scribner, C. E

Scribner, C. E

Scribner, C. E

July 1,1890
July 8,1890
July 22,1890
July 29,1890
July 29,1890
July 29,1890
Aug. 5,1890
Sept. 23,1890
Sept. 23,1890
Oct. 7,1890
Oct. 21,1890
Sor. 4,1590
Dec. 9, 1890
Dec. 9, 1890
Dec. 9, 1890
Dec. 9, 1890

442,342 Vaughn, J. W

443145 McQuarrie, J. L

444,10s Fambau, I. If

447,220 White, A. C

447,426 Gould, C. C

447,455 Beach, F. G

447,768

$\pm 48,322$

419,106

449,679

451,143

Francis, J. C

Digeon, L

Carty, J. J

Jacques, $\mathrm{W}$. $\mathrm{W}$ illiers-Stead, F. and Hedg- Apr. 28, 1891 man, E. R.

453,483 Jacques, Wr. W

Sundcen, D Volander S. B. June 9, 1891

Sundcen, A. D., Molander, S. B. Anderson, G. W. and Carlsen, A. M.

179. TELEPHONY.--101. Telephones-Srstems, AUtomatic.

\begin{tabular}{l|l|l|l|l}
435,295 & Ford, W. H & Aug. 26, 1890 447,918 Strowger, A. B
\end{tabular}

442,734 Smith, J. R. and Childs, W -.- Dec. 16, 1890

Mar. 10, 1891

179. TELEPHONY.-135. TELEPHONES-Srstens, Toll. 
191. ELECTRIC RAILWAIS.-1. Conductor Supports ANd 1nsulators.

\begin{tabular}{|c|c|c|c|c|c|}
\hline No. & NAME. & DAte. & No. & NAME. & DATE. \\
\hline 1 & Thomson, E. & July 1,1890 & 445,908 & Sharp, E. P & Feb. 3, 1891 \\
\hline & Hert & July 8,1890 & 446,483 & Thomson, $\mathrm{E}$ & Feb. 17, 1891 \\
\hline & Hallbauer, $A$. L. and Hiller, E. L & Ang. 5,1890 & 446,985 & Mackloskie, C. H. and Baker, & Feb. 24,1891 \\
\hline & Van Depoele, C. J & Aug. 26,1890 & 447,208 & Short, S. H _. L & Feb. 24, 1891 \\
\hline & Lieb, C. A & Sept. 2,1890 & & Short, S. H & Mar. 3, 1891 \\
\hline & Thomson, E & Sept. 2,1890 & 448,246 & Lieb, C. A & Mar. 17, 1891 \\
\hline & Tan Depoele, C. J & Sept. 30,1890 & 703 & Wirt, H. C & Mar. 24, 1891 \\
\hline & Wirt, H. C & Oet. 14,1890 & 449,943 & althy, I & Apr. 7, 1891 \\
\hline $\pm 1,31$ & $\begin{array}{l}\text { Marks, W. B.. Lauer, J. G. and } \\
\text { Ralston, R. R. }\end{array}$ & Nov. 25,1890 & & $\begin{array}{l}\text { Lieb, C. A } \\
\text { Lieb, C. A }\end{array}$ & $\begin{array}{l}\text { Apr. } 14,1891 \\
\text { Apr. } 14,1891\end{array}$ \\
\hline & Ritfield, E. H & Dec. $\quad 2,1890$ & 450,242 & Lieb, C. A- & Apr. 14, 1891 \\
\hline & Id, $F_{-}$ & Dec. 9,1 & & $\mathrm{Li}$ & Apr. 14,1891 \\
\hline $2 \pm$ & b. & Dec. 9,1590 & 45 & Lieb, C. A. and Le & Apr. 14, 1891 \\
\hline 3 & Ranasev, W. M & Dec. 16,1890 & 452,017 & Lieb, C. A & May 12,1891 \\
\hline 3 & Emmet, W. Le $\mathbf{R}_{-}$ & Dee. 23,1890 & 452 & Jennings, $B_{-}$ & May 19,1891 \\
\hline & t. IV. Le $\mathrm{R}_{-}$ & Dec. 30,1890 & 452,6 & B. & May 19,1891 \\
\hline & D & Jan. 6,1891 & $45 \%$ & $\mathrm{G} . \mathrm{K}$ & May 26,1891 \\
\hline & Mas & Jan. 6,1891 & 453,100 & W. E. and Macloskie, C, H & Mav 26,1891 \\
\hline & Vogler, $\mathrm{W}$ & Jan. 20,1891 & 453,326 & Ide, A. L ..... & June $2,189 \mathrm{i}$ \\
\hline & Vogler, W & Jan. 20, 1891 & $454, \pm 86$ & Lieb, C. A _..... & June 23,1891 \\
\hline & $\mathrm{S}_{-}$ & Jan. 20,1891 & & Lie & June 23,1891 \\
\hline & Foster, C. and Bevis, W. H & Feb. 3, 1891 & 455,107 & Munsie, J. F_.. & June 30,1891 \\
\hline
\end{tabular}

191. ELECTRIC RAlLWA YS.--2. Electric Locomotives,

\begin{tabular}{|c|c|c|c|c|c|}
\hline & McDougall, W. M & July 1,1890 & 584 & $a n, H$ & Oct. 28,1890 \\
\hline & Drown, F. E & July 1 & & & Nov. 11,1890 \\
\hline & Currie, S. C. C & July 8,1890 &, 686 & ker, I. F - & Nov. 18,1890 \\
\hline & Hunter, R. M & July \&, 1890 & 689 & ing, W. & Nov. 18,1890 \\
\hline & gue, F. J & July 8,1890 & & & Nov. 18,1890 \\
\hline & ------- & July 15,1890 & & , S. E & Nov. 18,1890 \\
\hline & Tा. $\mathrm{H}_{--}$ & July 15, & & & Nov. 25,1890 \\
\hline & hlin, J.F & uly 15 & & nger, & Nov. 25,1890 \\
\hline & son, J. W & July 15 & & R. $M \Gamma$ & Nov. 25,1890 \\
\hline & ooele, C. J J & July 15, & & $\mathrm{~F}$ & Dec. \\
\hline & $\mathrm{a}, \mathrm{E}$ & 1515 , & & ele, & Dec. 16 , \\
\hline & R. MI & July 22, ] & & 1 & Dec. 9 \\
\hline & W. jr---_- & July 22, & & L & Dec. 23, \\
\hline & R. & July 22 & & er, $\mathrm{R}$ & Dee. 30 \\
\hline & R. $\mathbf{M}$ & July 29 , & & . $M I_{-}$ & Dec. 30 , \\
\hline & & $1 y 29$, & & & \\
\hline & $\mathrm{L}_{-\ldots} \ldots \ldots \ldots$ & July 29 & & II & $1:$ \\
\hline & $\mathrm{B}$ & July 29,1 & & I, and Entz, J. B & Jan. 1 \\
\hline & & July 29 & & L. & Jan. 1 \\
\hline & . $\mathrm{I}$ & July 29 & & W., jr & Jan. 2( \\
\hline & $\mathbf{R}, \mathbf{M} \mathbf{I}_{\ldots}$ & July 29 & & C: $\mathrm{p}^{\prime}$ & Jan. 20 \\
\hline & E. & Ang. 5, & & $\mathrm{M}$ & Jan. 20 \\
\hline & E. and Spencer, & Aug. 5, & & $\mathrm{ky}, \mathrm{C}$ & Feb. \\
\hline & ------1 & Aug. 12, & & & $\mathrm{Fel}$ \\
\hline &.$Y_{-}$ & A & & $\mathbf{R}$ & Feb. 1 \\
\hline & R. $\mathrm{M} \ldots$ & & & $\mathrm{A}_{-}$ & Feb. 17 \\
\hline & & 12 & & E. M & Feb. 1 \\
\hline & 1 & .12, & & & $\mathrm{Fe}$ \\
\hline & . & & & F. O & $\mathrm{M}$ \\
\hline & & & & & MI \\
\hline & & & & $\mathrm{C}$ & \\
\hline & S. & & & & \\
\hline & $\mathrm{H}_{-}$ & g. 26, & & U. J- & MI \\
\hline & $\mathrm{H}$ & & & & M] \\
\hline & & t. 2 & & & $\mathrm{MI}$ \\
\hline & $V_{-}$ & pt. 2, & & id Lange, $\mathrm{H}$. & $\mathrm{Ma}$ \\
\hline & $\mathrm{E}_{-}$ & & & or & $\mathrm{Ma}$ \\
\hline & & & & & Mar. 31, \\
\hline & & & & & Apr. 7, \\
\hline & & & & & Apr. 7 \\
\hline & F & t. 16 & & C. J & Аpr. 14, 1891 \\
\hline & & & & & \\
\hline & & & & & \\
\hline & H & t. 31 & & $1, \mathbf{J}$ & Apr. 2 \\
\hline & & & & & Ap \\
\hline & $\mathrm{Pa}$ & Se $\Rightarrow$ & & & Apr. 2 \\
\hline & Dean, F. W & Oct. 28,1890 & 51,491 & nignt, & May \\
\hline
\end{tabular}


191. ELECTRIC RAILWAYS.-2. EleCtRIC Locomotives-Continued.

\begin{tabular}{|c|c|c|c|c|c|}
\hline No. & NAME. & DATE. & No. & NaMe. & DATE. \\
\hline 451,980 & Short, S. H & May 12, 1891 & 452,622 & Short, S. H & May 19,1891 \\
\hline 451,981 & Short, S. H - & $M_{\text {iay }} 12,1891$ & 452,992 & Dobbie, R. S & Iay 26,1891 \\
\hline 452,005 & Short, S. H & May 12,1891 & 453,167 & Eiekemeyer, R & May 26,1891 \\
\hline 452,035 & Short, S. H - & May 12,1891 & 453,216 & Shawhan, J. F & June 2,1891 \\
\hline 452,036 & Short, S.H & May 12, 1891 & 454,008 & Short, S. H & June 9,1891 \\
\hline 452,176 & Christiansen, $\mathbf{J}_{-}$. & Мay 12,1891 & 454,020 & Bentley, E. M. & June 16, 1891 \\
\hline $452,+22$ & Blackwell, F.O & May 19,1891 & $\pm 54,021$ & Bentley, E. M. & June 16,1891 \\
\hline 452,423 & Blackwell. F. O $\ldots$ & May 19,1891 & 454,888 & Stephenson, J & Juиe 30,1891 \\
\hline 452,621 & Short, S. H _...- & May 19,1891 & & & \\
\hline
\end{tabular}

191. ELECTRIC RAILWAYS.- 3. STSTEMS.

431,711 Short, S. H

432,095

434,687

440,976

441,828

442,140
Swart, W. D

Tan Depoele, C. J.

Tan Depoele, C. J

Dewey, M. IV

Salsoldt, F. IT
July $\&, 1890$ July 15,1890 Aug. 19, 1890

Niv. 18. 1890 Dec. ㄹ, 1590 Dee. 9,1890
442,365 444,740 445,479 448,328 448,598 450,193
Dewey, M. II

Bentley, E. II

Short, s. $\mathrm{H}$

Jan. 27,1891

Eutz, J. B_-_._._._._..... Mar. 17, 1891 Wheeler, S. S. and Bradley, C. S Mar. 17, 1891 Pobinson, C. A _............ Apr. 14, 1891

191. ELECTRIC RAILWAYS.-1. SYsteMs-BatTERY.

4:33,360 McLauglilin, J. F

433,551

434,579 434,580

434,581

$434,58 \cdot 2$

434,868

438,192

$439,-237$
Chamberlain, J. C

Corning, F. G

Corning, F. C

Curning, F. G

Corning, F. G

Corning, F. G

Shawhan, J. F

Corning, F. G.
July 29,1890

Aug. 5, 1890

Aug. 19, 1890

Ang. 19,1890

Aug. 19, 1890

Aug. 19, 1890

Aug. 19, 1890

Oct. 14,1890

Oet. 28,1890
$439,39.5$

440,362

441,305

442,744

446,613

446,817

$+47,230$

455,019
Fleiseher, R. J

Nourse, J. K. P

Hunter, R. M

Stephenson, J

Rie, F. B

Hunter, R. M

Chamberlain, J.

Ingralıam, A. A.
Oct. 28,1890 Nov. 11, 1890 Nov. 25,1890 Dee. 16,1890 Feb. 17, 1891 Feb. 17, 1891 Feb. 24, 1891 June 30, 1891

191. ELECTRIC RAILWA IS.-5. SYsTEMS-CONDUT.

431,519 Hunter, R. M

431,633

431,634

432,204

432,416

432,571

432,670

$+32,941$

433,409

$433,5 \pm 1$

434,030

434,036

434,389

434,390

435,536

435,662

435,479

436,923

437,720

437,953

439,069

439,262

439,597

440,596

440,597

440,780

440,781

440,906

441,210

Hunter, R. M

Wehrle, J. H

Lrneh, J -

Jennings, B

Jennings, B

Wehrle, J. H

Hunter, R. MI

Hunter, R. II

Curtis, H. U

Iunter, R. MI

Hunter, R. II

Libbey, H. iv

Reed, E. II

Welirle, J.H

Hunter, R. II

Westerland, L

Hunter; R. M

Hunter, R. M

Hunter, R. M

Verstraete,

Verstraete,

Trott, $\mathrm{S}$

Wheless, II
MeLaughlin, J. F

Keithley, H. B

July 29,1890

Thobe, A. A. and Embles, W-- Aug. 5, 1890 Hunter, R. M _._._. Aug. 12, 1890
July $1,1890 \quad 441,212$

July 8,1890

July 8,1890

July 15.1890

July 15,1890

July 22, 1890

July 22,1890

July 22,1890

lug. 12,1890

Aug. 12,1890

Aug. 12, 1890

Sept. 2, 1890

Sept. 2, 1890

Sep1. ? 1890

sept. 23,1890

(oct. 7,1890

Oet. $\quad \overline{1}, 1890$

Oet. 21,1890

Oet. 28,1890

Oct. 2s, 1890

Yor. 11, 1890

Nor. 11,1890

Үor. 18,1890

Nor. 18,1890

Nor. 18, 1890

Nov, 25, 1890
411,221

441,258

$441,5 i 1$

441,572

441,764

444,687

$445,63 \mathrm{t}$

445,674

446,376

416,417

446,419

$4-46,420$

$4+6,475$

47,332

$4+7,038$

448,461

tts 38

449,797

451,326

451,692

451,815

452,160

452,611

452,920

454,177

454,178

454,022

454,023
IVheless, M

Wheatley, S. E-

Libley, H. IT

Libbey, H. W

Jones, HI

Blackwell, J. I

Bentley, E. II

Hunter, R. MI

Bentley, E. MI

Bentley, E. II

Bentley, E. M

Bentley, E. II

Seibert, $\mathrm{X}$

Knight, W. H

Medbery, H. J

Stewart, R. A

Poeock, F. A

Hunter, R. II

Jennings, B

Hunter, R. II

Ford, II. H

Blaekwell, F. O

Iitehell, E. IV

Hunter, R. M

Miller, J. J

Niller, J. J

Bentley, E. M

Blaekwell, F, O
Nov. 25, 1890 Nov. 25, 1890 Nov. 25, 1890 Tov. 25, 1890 Nor. 25, 1890 Dee. 2, 1890 Jan. 13, 1591 Feb. 3, 1891 Feb. 3, 1891 Feb. 10, 1891 Feb. 17, 1891 Feb. 17, 1891 Feb. 17, 1891 Feb. 17, 1891 Mar. 3, 1891 Mar. 3, 1891 Mar. 17, 1891 Mar. 24, 1891 Apr. 7, 1891 Apr. 28, 1891 May 5, 1891 May 5,1891 May 12,1891 Vay 19,1891 May 26, 1891 June 16, 1891 June 16, 1891 June 16, 1891 June 16, 1891

191. ELECTRIC RAILITA YS.6- Systemis-Closed Condeit.

432,673 Mansfield, $F$

432,674 Mansfield, $\mathrm{F}$

$434, \pm 10$

Tan Depoele, C. J 
191. ELECTRIC RAILWAYS-6. SrsteMs-Closed ConduIT-Continued.

\begin{tabular}{|c|c|c|c|c|c|}
\hline No. & NAuE. & DATE. & No. & NAMF. & DATE. \\
\hline $\begin{array}{l}435,487 \\
437,358 \\
140,8 \div 2 \\
141,214 \\
411,216 \\
143,084\end{array}$ & $\begin{array}{l}\text { Elliott, W. R } \\
\text { Hardiug, C. K } \\
\text { Bremer, W. G } \\
\text { Wheless, MI. } \\
\text { Wheless, M. } \\
\text { Bentley, E. M }\end{array}$ & $\begin{array}{l}\text { Sept. 2, } 1890 \\
\text { Sept. } 30,1890 \\
\text { Nov. } 18,1890 \\
\text { Nor. 25, } 1890 \\
\text { Nov. 25, } 1890 \\
\text { Dec. 23, } 1890\end{array}$ & $\begin{array}{l}443,451 \\
450,172 \\
450,173 \\
451,637 \\
451,691\end{array}$ & $\begin{array}{l}\text { Hunter, R. M } \\
\text { Mansfield, F } \\
\text { Mansfield, F } \\
\text { Harding, C. K } \\
\text { Holland, J }\end{array}$ & $\begin{array}{l}\text { Dec. } 23,1890 \\
\text { Apr. 14, } 1891 \\
\text { Apr. } 14,1891 \\
\text { Mray } 5,1891 \\
\text { Hay } 5,1891\end{array}$ \\
\hline
\end{tabular}

191. ELECTRIC RA1LWAYS.-7. Sxstems-Circuits, Multiple Arc.

\begin{tabular}{|c|c|c|c|c|c|}
\hline 40 & Hnn & July 8, & 4 & II & Nor \\
\hline & & July 15,1890 & & & N \\
\hline & II & Aug. 5,1890 & 441 , & 1 & No \\
\hline & R. M & Aug. 12,1890 & & tley, S. E. & Yov. 25,1890 \\
\hline & $\pi$ & Sept. 2,18 & & M. J & De \\
\hline & & Oct. 14 & & $\mathrm{H}$ & $\mathrm{Fe}$ \\
\hline & S1 & Oct. 14, & & $\mathrm{H}$ & $\mathrm{Ma}$ \\
\hline & $\mathrm{H}$ & Oct. 21,1 & & T. A & \\
\hline & $\mathrm{T}$ & Nor. 11,1 & & S. F. I & Mar. 31, \\
\hline 1,1 & Wheless, : & Nov. 25,1890 & 451,154 & Hunter, R. M & Apr. 28, \\
\hline
\end{tabular}

191. ELECTRIC RAILWAYS.-8. STateVIS-Circuits, Series.

$\begin{array}{ll}439,429 & \text { Richter, C } \\ 439,967 & \text { Dewey, M. W. W }\end{array}$

191. ELECTRIC RAILWAYS.-9. STSTEMS-CONVERTER.

431,482 Ries, E. E.

43.,684 Tan Depoele, C. J

$434,68.5$ Tan Depoele, C. J
July 1,1890

Aug. 19,1890

Aug. 19,1890 $434,686^{\circ}$ Van Depoele, C. J

$44 i, 215$ Van Depoele, C. J.
Aug. 19,1890 Feb. 24,1891

191. ELECTRIC RAILIHAYS.-10. SYSTEMS-DOUBLE.

452,099 Dewer, M. W

May 12, 1891

191. ELECTRIC RAILW Y YS.-11. STsteMS-OVERHFAD.

431,092

431,093

431,094

431,095

432,581

436,425

436,944

437,613

438,847

438,894

439,662

439,746

$40,59.5$

442,623

412,081

$445, \pm 09$

445,515

$445,9.52$

416,418

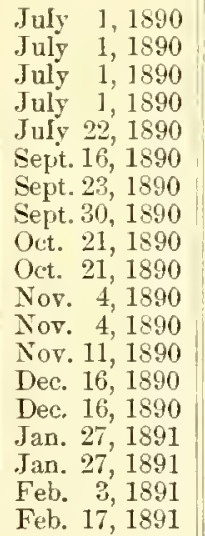

446,833

477,283

447,885

48,505

448,711

448,831

449,792

450,074

450,586

450,613

450,687

451,211

451,696

452,576

453,710

454,881

454,903

455,233

455,234
Hunter, R. M

Hunter, R. MI

Luce, C. J

Crow, B. F

Farnham, I. H

Eickemever, R

Bentley, E. M

Hunter, R. M

Hunter, R. M

Culver, F. S

Thomson, E

Macrae, $\mathbf{R}$.

Kuehnle, J

Kuehnle, J

Jones, J

Scott, G. J

Fletcher, J. R

Munsie, J. F

Munsie, J. F
Feb. 17, 1891 Feb. 24, 1591 Nar. 10, 1891 Mar. 17, 1891 Mar. 24, 1891 Mar. 24, 1891 Apr. 7, 1891 A pr. 7, 1891 Apr. 14, 1891 A pr. 21, 1891 A pr. 21, 1891 Apr. 28, 1891 May 5, 1891 May 19, 1891 June 9, 1891 June 39, 1891 June 30, 1891 J une 30,1891 June 30, 1891

191. ELECTRIC RAILIVAYS. - 12. Systems-OverhedD, Trolleys.

Andersen, J. M Miller, W. C. Hunter, R. M Hunter, R. II Sprague, F. J
July 8, 1890 July 15, 1890 July 22, 1890 July 29, 1890 July 29, 1890
433,611 433,839 434,276 434,682 435,011
Bain, F

Wheeler, F. C

Hunter, R. M

Stevens, W. L. and Wescott, E.J Aug. 19, 1890

Cairns, S. D -................... Ang, 1890 
191. ELECTRIC RAILWAYS.-12. Srstems-Overhead, Trollexs-Continued.

\begin{tabular}{|c|c|c|c|c|c|}
\hline No. & NAME. & DATE. & No. & NAME. & DATE. \\
\hline 435,166 & Atwood, L- & Aug. 26,1890 & 444.566 & Hunter, R. M & Jan. 13,189$]$ \\
\hline 435 & Duggan, J.A & Sept. 2,1890 & 444,871 & Baker, I. F. and Booth, R & Jan. 20, 1891 \\
\hline 436,571 & Ainslie, D. A. & Sept. 16,1890 & 44,893 & Blades, H. H & Jan. 20,1891 \\
\hline 436,874 & Hoppes, J. J & Sept. 23,1890 & 446,428 & Cahoon, J. B. and Baker, I. F & Feb. 17, 1891 \\
\hline 437,534 & Van Depoele, C. J & Sejt. 30,1890 & 446,931 & Short, S. H & Feb. 24,1891 \\
\hline 961 & Baker, I. F _..... & Oct. 7,1890 & 447,632 & Cavert, W. J. and Wiswall, W.P & Mar. 3,1891 \\
\hline 19 & Andersen, J. M & Oct. 14,1890 & 448,172 & Wightman, M. J & Mar. 10,1891 \\
\hline & n-n-n-n & Oct. 14,1890 & 41 & Wightman, NI. J & Mar. 10,1891 \\
\hline 404 & Chadbourne, A. $\mathrm{H}_{--\ldots-\ldots .}$ & Oct. 14,1890 & 448,618 & Hunter, R. MI ...... & Mar. 17,1891 \\
\hline 439,092 & Bardwell, A. F & Oet. $2 S, 1590$ & 449,226 & Witchell, A. WV & Mar. 31,1891 \\
\hline 182 & 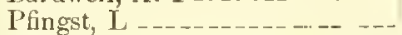 & Oct. 28,1890 & 449,490 & Mitchell, A. W & Mar. 31,1891 \\
\hline 192 & Rowell, B. C. and Galloupe, F. E & Oct. 28,1890 & 39 & $\mathrm{~ns}, \mathrm{~B} . \mathrm{J}$ & Mar. 31,189 \\
\hline 439,308 & G. S - & Oct. 25,1890 & 449,886 & Robinson, F. and Lander, P. IV.J & Apr. 7, 1891 \\
\hline 916 & tlesey, J, T_......... & Nov. 4,1890 & 450,184 & Palmros, $\mathrm{A}$ & Apr. 14,1891 \\
\hline & , L. S. & For. 4,1890 & 450 & Hickley, A. S & Apr. 14,1891 \\
\hline 84 & Brooks, H. H & Nov. 11, 1890 & 150,189 & Jackson, WV. E., jr.-. & Apr. 14,1891 \\
\hline 89 & ing, R. D _........... & సov. 18,1890 & 450,683 & Short, S. H & Apr. 21,1891 \\
\hline 814 & C. $A_{-}$ & Nov. 18,1890 & 450,853 & Webber, H. A & Apr. 21, 1891 \\
\hline 665 & - - - - - - & Nor. 18,1590 & 402 & er, R. M & A pr. 28,1891 \\
\hline 122 & 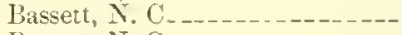 & Nor. 25,1890 & 45 & $s, \mathbf{R} . \mathbf{M}$ & Miay 12, 1891 \\
\hline 441,123 & Bassett, $\mathrm{X}, \mathrm{C} \ldots$ & Nov. 25,1890 & $152,5+2$ & Conk, D. I ....... & May 19,1891 \\
\hline 441,753 & Enmmet, II. Le R & Dec. 2,1590 & 453,093 & Sullivan, J & May 26,1891 \\
\hline 002 & Adams, A. W .... & Dee. 2,1890 & $43 \overline{7}$ & r, R. M & June 16,189 \\
\hline 04 & Ramsey, II. M & Dec. 16,1890 & 454,522 & Larkin, G. H. and Tomkin & June 23,189 \\
\hline 152 & , R. MI & Dec. 23,1890 & 454,532 & Verstraete, E -.--_..... & June 23,189 \\
\hline 444,397 & Hunter, R. M & Jan. 6,1691 & 454,536 & Duncan, $W_{-}$ & June 23,189 \\
\hline 444,447 & Lieb, C. A & Jan. 13,1891 & & & \\
\hline
\end{tabular}

191. ELECTRIC RAILWAYS-13. SYsteMS-TELPHER.

434,871 Hunter, R. II

452,741 Weems, D. G

\section{Aug. 19, 1890}

May 19,1891
453,031 Baker, E

II ay 26,1891

191. ELECTRIC RAILMAYS.-14. SYsteMS-TELPHER, Solexom.

433,381 Williams, J. T _............ July $29,1890 \quad 438,870$ Torld, A. E

436,281 Williams, J. T.

138,564 Swift, H. E.. \begin{tabular}{r|rr} 
July 29,1890 & 438,870 & Tord, A. E \\
Sept. 9, 1890 & 41,250 & Manaydier, J. E
\end{tabular}

Oct. 21,1890 Oct. 14,1890
Nor. 25,1890

2. APPAREL. 1. CorTING.

431,999 Salisbury, W. S

July 8,1890

14. BRIDGES. - 3. GIRDers and Coluniss.

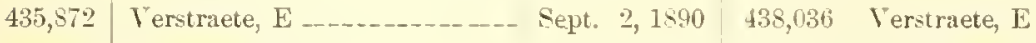

Oct. 7,1890

14. BRIDGES.-10. Towers.

434,639 Maxwell, D

Aug. 19, 1890

18. CAOUTCHOUC AND MINOR PLASTICS. -11. Insulatixg Materials.

431,104 Cheever, J. H__._._._._._. July 1, 1890

433.215 Rabinoricz, I

Fottrell, J . Aug. 5, 1890

436,733 Easton, J. IT _................. Sept. 16, 1890

437,044 Rawson, F. L_-_-_-_-_-_-_-_- Sept. 23, 1890

438,309 Edison, T. A

438,311 Enholm, O. A.............. 14, 1890

438,313 Enholm, O. A _............. Oct. 14, 1890
438,698 Menuez, A. E.

439,796 Bottome, T. D

440,391 Blaisdell, F. E

452,760 Salathe, $F$

452,765 Salathé, $F$

454,547 Sperry, A. TV

454,548 Sperry, A. W
Oct. 21,1890

Nov, 4, 1890

Nor. 11,1890

May 19, 1891

Nay 19,1891

June 23,1891

June 23, 1891 
18. CAOUTCHOUC AND MINOR PLASTICS.-7. Miscelalaneous Compositions.

\begin{tabular}{|c|c|c|c|c|c|}
\hline No. & NAME. & DATE. & No. & NaME. & DATE. \\
\hline $\begin{array}{l}116,502 \\
\pm 16,573\end{array}$ & $\begin{array}{l}\text { Wright, E. G } \\
\text { Madden, A. F }\end{array}$ & $\begin{array}{l}\text { Feb. } 17,1891 \\
\text { Feb. } 24,1891\end{array}$ & 452,599 & Dooley, T. B............ & May 19, 1891 \\
\hline
\end{tabular}

18. CAOUTCHOUC AND MINOR PLASTICS.-9. Vulcanizing Apparatus.

\begin{tabular}{l|l}
\hline 40,395 & Cobb, H. B
\end{tabular}

20. CARPENTRY.-24. SLIDING Doors.

$4 \pm \bar{i}, \overline{5} 05$ Coburn, L

22. METAL FOUNDING.-39. Ix Molded Molds, Wheels.

$445,319 \mid$ Lenhart, P. F

23. CHEMICALS.-27. ELECTROLYsIS.

\begin{tabular}{|c|c|c|c|c|c|c|}
\hline 412,332 & Roberts, I. L & Dec. & 9,1890 & 447,585 & Schroeder, G. G & Mar. 3, 1891 \\
\hline 412,334 & Roberts, I. L & Dec. & 9,1890 & 448,541 & Parker, T. and Robinson, A. E- & Mar. 17,1891 \\
\hline 442,396 & Roberts, I. L _ & Dec. & 9,1890 & 450,108 & Le Sueur, E. A & Apr. 7,1891 \\
\hline 412,594 & Roberts, I. L & Dec. & 9,1890 & 450,104 & Le Sueur, E. A & Apr. 7,1891 \\
\hline
\end{tabular}

25. CLAY AND POTTERY.-S. DraxN TIles.

45̃1,9t1 La Point, T. T., Flanagan, J. H. and Thompson, C. A

28. CORDAGE。-14. WaRPING.

432,040 Haslam, J. P

25. CORDAGE.-31. Warp, Stop Motion.

443,674 Goldschmidt, $\mathrm{P}$

Dec. 30,1890

39. FENCES.-36. Rallway Gates-Automatic Mast.

443,729 Seele, J. W

Dec. 30,1890

39. FENCES.-38. RaIlway Gates-Electric.

\begin{tabular}{|c|c|c|c|c|c|}
\hline $\begin{array}{l}438,620 \\
441,032 \\
442,114\end{array}$ & $\begin{array}{l}\text { Gillette, H } \\
\text { Sheldon, W. D } \\
\text { Gillette, H }\end{array}$ & $\begin{array}{l}\text { Oct. } 21,1890 \\
\text { Nov. } 18,1890 \\
\text { Dec. } 9,1890\end{array}$ & $\begin{array}{l}443,732 \\
444,825\end{array}$ & $\begin{array}{l}\text { Steele, J. W } \\
\text { Gillette, H }\end{array}$ & $\begin{array}{l}\text { Dec. } 30,1890 \\
\text { Jan. } 20,1891\end{array}$ \\
\hline
\end{tabular}

40. ADVERTising.-2. Automatic Devichs.

\begin{tabular}{|c|c|c|c|c|c|}
\hline 437,905 & Levi, $\mathrm{I}$ & Oct. $\quad 7,1890$ & 453,190 & Jackson, S. D.L & June 2,1891 \\
\hline
\end{tabular}

40. ADVERTISING.-14. Signs. 
41. Fine arts.-8. Exgraving Machines.

\begin{tabular}{|c|c|c|c|c|c|}
\hline No. & NAME. & Date. & No. & NaMe. & DAte. \\
\hline 431,578 & Guerrant, G. M _............ & JuIy 8,1890 & 448,404 & Amstutz, N.S & Mar. 17,1891 \\
\hline \multicolumn{6}{|c|}{ 41. FINE ARTS-17. THEATER APPLIANCES. } \\
\hline $\begin{array}{l}442,796 \\
446,797\end{array}$ & $\begin{array}{l}\text { Burgess, N } \\
\text { Burgess, N }-\ldots .\end{array}$ & $\begin{array}{l}\text { Dee. } 16,1890 \\
\text { Dee. } 16,1890\end{array}$ & 443,991 & Ellis, S. R & Jan. 6,1891 \\
\hline
\end{tabular}

42. FIRE ARMS.-25. Ball Traps.

$433,55 \pm$ True, L. L

42. FIRE ARUS.-12. LOCKS.

439,055, Von Dérschau, A

Oct. 21,1890

46. GAMES AND TOYS. 45. TOYS-FigORE WHEELED.

454,570 Moore, G. R

48. GaS-25. Carferetors-Gas and Air Mixers.

451,037 Frost, E. J

Apr. 28, 1891

49. GLASS - 7. MoLDS.

453,575 Atterbury, T. B

June 9,1891

49. GLass.-10. Plate and Window.

433,254 Niles, H. B. July 29, 1990

51. GRINDIAG AXD POLISHING.-4. Metal-Curved Surfaces.

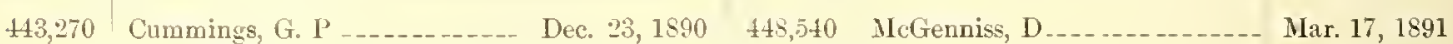

53. HARDWARE MAKIN゙G.-9. MIECELLANEOCS.

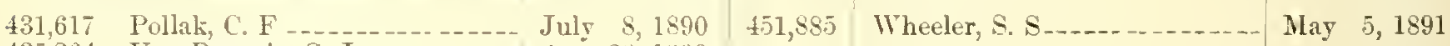
$435,26^{4}$ Tan Depoele, C. J. Aug. 26, 1890

57. HOISTING - 34. BLOCK AND TACKLE.

$\$ 2, \$ 15$ Brady, T. H Dec. 9,1890

58. HOROLOGY.-2S. ClOCKS-EleCTric.

437,203 Hunter, G. E. and Corthell, F. H Sept. 30, 1890 437,204 Hunter, G. E. and Corthell, F. H Sept. 30,1890 437,205 Hunter, G. E. and Corthell, F. H Sept. 30, 1590 437,206 Hunter, G. E. and Corthell, F. H Sept. 30, 1890 438,314 Gipperich, A _-__-_-_oct. 14, 1890 439,838 Drson, J. H $\ldots \ldots$ Nor. 4,1890

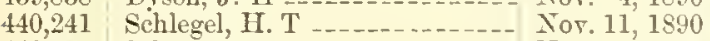
$440, \pm 41$ Schwartz, F__._........... Nov. 11, 1890 443,291 Maýrhofer, C _._._......... Dec. 23, 1890 444,433 Hammer, E. G............ Jan. 13, 1891 441,482 Bradley, W.T. and Packer, W.N Jan. 13, 1891 445,023 Lefebrre, E._._........... Jan. 20, 1891 445,073 Packer, W. N. and Bradley,W.W Jan. 20, 1891
446,801 Van de Plancke, D. and G-..-- Feb. 17, 1891 447,105 Dudley, W. J 445,291 Chase, H. A _................ Mar. 17, 1891 48,998 Scales, W. S 448,999 Scales, IV. S - Mar. 24, 1891 452,299 Wiseman, A. G _........... May 12, 1891

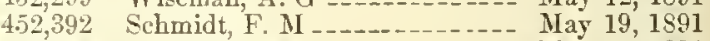
452,424 Church, A. MI_.._._._...... May 19, 1891 452,955 Prentiss, H. S................. May 26, 1891 452,956 Prentiss, H. S.............. May 26, 1891 455,041 Von Hefner-Alteneck, F _..._- June 30, 1891 455,055 Ramsay, W.............. June 30, 1891 
58. HoRology.-29. Clocks-Pxeunatic.

\begin{tabular}{|c|c|c|c|c|c|}
\hline No. & Name. & DATE. & No. & NAME. & DATE. \\
\hline 438,767 & Popp, $V_{-.}$ & & & & Oct. 21,1890 \\
\hline
\end{tabular}

58. HOROLOGY.-15. Time Alarus.

\begin{tabular}{|c|c|c|c|c|c|}
\hline 21 & Deane, IT. H & Aug. 26,1890 & 445,341 & Striemer, $\mathrm{A}$ & Jan. 27,189$]$ \\
\hline 53 & Striemer, $\mathbf{A} \ldots \ldots$. & Oet. 21,1890 & 448,325 & Edgerton, E. M _ & Mar. 17, 1891 \\
\hline & Tiedemann, M. W & Dec. 2,1890 & 448,712 & Gallagher, J. J. & Mar. 24,189 \\
\hline
\end{tabular}

58. Horolog T--16. The Chects.

\begin{tabular}{|c|c|c|c|c|c|}
\hline $\pm 35,185$ & Park, H.S P $_{n} \ldots$ & Aug. 26, 1890 & 445,113 & Hörenz, F & Jan. 20,1891 \\
\hline 435,582 & Fuller, F. L & Sept. 2,1890 & 451,666 & Gee, E. C & May 5, 1891 \\
\hline $413, \pm 35$ & Fuller, I. D & Dec. 23,1890 & 452,894 & Bundy, IV. L ... & May 26,1891 \\
\hline $\pm 13,787$ & Ongley, C. E & Dec. 30,1890 & 453,230 & Bundy, M. I $\ldots$ & June 2,1891 \\
\hline $113-88$ & Ongles, C. E, E & Dec. 30,1890 & & & \\
\hline
\end{tabular}

58. HOROLOGY.-26. WaTCH STOPs.

450,966 Matthews, F.A

67. LAMPS AND GAS FITTINGS.-5. DROP LIGHTS AND HANgers.

\begin{tabular}{|c|c|c|c|c|c|}
\hline 434,038 & White, A. B- & Aug. 12,1890 & 444,424 & Dawes, A. & Jan. 13,1891 \\
\hline 437,883 & Brady, T. H & Oet. 7,1890 & 445,549 & Fitch, G & Feb. 3, 1891 \\
\hline 438,779 & Newton, S. O. and M. A & Oct. 21,1890 & 446,037 & Titus, J. E & Feb. 10,1891 \\
\hline 9,023 & Matson, D. B $\ldots$ & Oct. 21,1890 & 448,865 & Smith, C. G & Mar. 24, 189 \\
\hline 1,248 & Lynch, T. J & Nov. 25,1890 & 449,902 & Easton, J. W --.- & Apr. \\
\hline 472 & Batchelder, G. L _-_-- & Dec. 9,1890 & 451,108 & Danals, C. A. and Perry, W. $\mathrm{O}_{-}$ & Apr. 2 \\
\hline 443,097 & Kim, G. II & Dec. 23,1890 & 451,202 & Thomson, D. P & Apr. 28, 1891 \\
\hline & 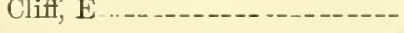 & Jan. 6,1891 & 451,996 & Dow, IV. E --- & May $1:-, 18$ \\
\hline
\end{tabular}

67. LAMPS AND GAS FITTINGS.-58. Drop Lights-SpRING Drun.

452,799 Matteson, J. A

67. LAMPS AND GAS FITTINGS.-8. GAS FITTINGS.

442,270 Iarshall, $\mathrm{N}$

Dee. 9,1890

67. LAMPS AND GAS EITTINGS.-77. GLOBES.

452,638 Gärtner, A. F. and Harris, J. C- May 19, 1891 452,701 Gärtner, A. F. and Harris, J. C_ May 19, 1891

67. LAMPS AND GAS FITTINGS.-51. GAS BURNERS-INCANDESCENT.

450,099 Fahnehjelm, O. B Apr. 7,1891

67. LAMPS AND GAS FITTINGS.-94. GUARDS.

442,071 Mino, J .

Dee. ?, 1890

67. LAMPS AND GAS FITTINGS.-78. Harps and Hangers.

$453,002]$ Hamilton, J. E. 
67. LAMPS AND GAS FITTINGS.-22. LaMP SHADES.

\begin{tabular}{|c|c|c|c|c|c|}
\hline No. & Name. & Date. & No. & NAME. & DATE. \\
\hline $\begin{array}{l}432,826 \\
442,577 \\
443,169\end{array}$ & $\begin{array}{l}\text { Goehst, J. H } \\
\text { Fraser, W. S } \\
\text { Simpson, J. T. }\end{array}$ & $\begin{array}{l}\text { July } 22,1890 \\
\text { Dee. 9, } 1890 \\
\text { Dec. } 23,1890\end{array}$ & $\begin{array}{l}445,259 \\
448,741 \\
449,407\end{array}$ & $\begin{array}{l}\text { Curtis, W. B. and Himrod, C } \\
\text { Mueller, E. T } \\
\text { Easton, J. W }\end{array}$ & $\begin{array}{l}\text { Jan. } 27,1891 \\
\text { Mar. 24, } 1891 \\
\text { Mar. 31, } 1891\end{array}$ \\
\hline
\end{tabular}

67. LAMPS AND GAS FITTINGS-21. LAMP POSTS.

439,773 Dinn, P. J

Oct. 21,1890

67. LAMPS AND GAS FITTINGS.26. Locorotive Head Liants.

449,719 Ward, B. B -

67. LAMPS AND GAS FITTINGS-72. ReFLeCtors.

449,403 Cooley, F Mar. 31, 1891

67. LAMPS AND G.1S FITTINGS.67. SHADE SUPPORTS.

413,563 Bergmann, $\mathrm{S}$

Dee. 30,1890

67. LAMPS AND GAS FITTMGS-73. Street LAMP HaNGers.

454,166 Dillon, T June 16, 1891

70. LOCK's AND LATCHES.-26. TIIE Locks.

$\pm 54,897$ Adams, G. IV June 30,1891

72. MASONRY.-20. STONE AND BRICK SETTINA.

431,630 Wallace, $\mathrm{T}$. July 8,1890

73. MEASURING IXSTRUMENTS.-S6. CAsI IxDICATOR.

$4+3,475$ Bundy, II. I $449,10 \mathrm{~S}$ Desant, IV. F.

73. MEASURIVG INSTRUMEXTA.-15. DYNAMOMETERS.

452,941 Scribner, C. E. May 26, 1891

73. MEASURING INSTRUMENTS.-09. Fare Registers.

451,611 Allison, M. H. May 5, 1891

73. MEASURING INSTRUMENTS-76. LETTER BoxES.

433,560 Detwiller, W. B

73. MEASURING INSTRUMENTS-61. Measuring PUMps.

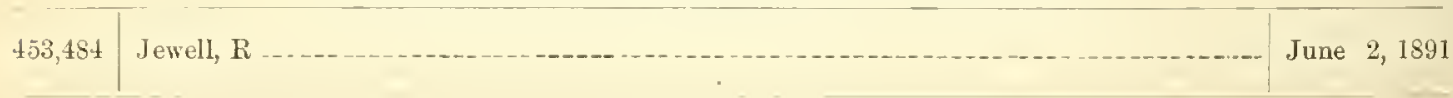


73. MEASURING INSTRUMENTS.-62. Measuring Vessels.

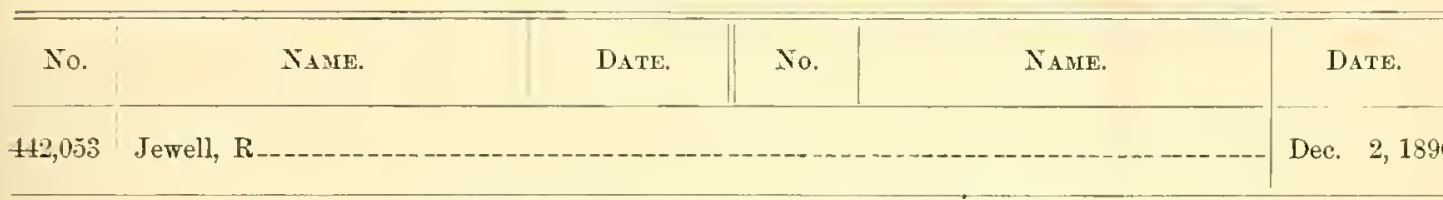

73. Measuring Instrumeyts.-32. Pyrometers.

441,488 Hensley, W. S. and Grindley, W. H

73. MEASURING INSTRUMENTS-34. RECORDERS.

\begin{tabular}{l|l|l||l|l|l|}
\hline 443,360 & Edson, J. B................ & Dec. 23, 1890 & 477,594 & Williams, H. B ............- Mar. 3, 1891 \\
\hline
\end{tabular}

73. MEASURING INSTRUMENTS-90. ReCORDERS-PAsSENGER.

\begin{tabular}{l|l}
\hline 432,003 & Torrey, $A$
\end{tabular}

73. MEASURING INSTRUMENTS.-35. REgISTERS.

\begin{tabular}{|c|c|c|c|c|c|}
\hline $434, \overline{8} 84$ & Drew, W. W & Aug. 19, 1890 & 444,358 & Jewell, $R_{-\ldots}$ & Jan. 6,1891 \\
\hline
\end{tabular}

73. LEASURING INSTRUMENTS.-38. SACK SCALES.

417,385 Van Hoesen, G. D. Mar. 3, 1891

73. MEASURING INSTRUMENTS.39. Scale Beams.

436,910 Snelgrore, W.

466,208 Röver, A. B \begin{tabular}{|l|l|l|l} 
Sept. 23, 1890 & 449,468 & Davis, J. V.............. & Mar. 31, 1691 \\
\hline Feb. 10, 1891 & & & \\
\hline
\end{tabular}

73. MEASURING INSTRUMENTS.-69. ShIP's Logs.

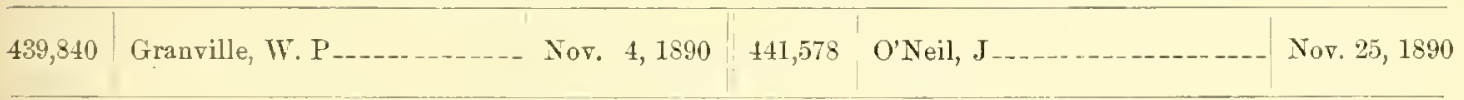

73. MEASUPING INSTRUMENTS.-43. SPEed MEAsUres.

\begin{tabular}{l|l|l|l}
\hline 416,488 & Waring, J J & & \\
\end{tabular}

73. MEASURIYG INSTRUMENTS.-85. TELETHERMOMETERS.

455,268 Wiesebrock, E. W

June 30, 1891

73. MEASURING INSTRUMENTS,-51. 'Testing Mähines.

434,181 Foote, T. II

73. MEASURING INSTRUMENTS.-52. THERMOMETERS.

434,774 Fox, E. M-Aug. 19, $1890 \quad 442,879$ Haight, H. J .

Dec. 16,1590

73. MEASURING INSTRUMENTS.-65. TIIERMostats.

448,401 Wilson, J. C -

73. MEASUPING INSTRUMENTS. -82. Water Gages-Froat.

444,295 Fuller, I. A A 
74. MACHINE ELEMENTS.-7. GEARING.

\begin{tabular}{|c|c|c|c|c|c|}
\hline No. & NAME. & Date. & No. & NAME. & Date. \\
\hline $\begin{array}{l}431,811 \\
437,259\end{array}$ & $\begin{array}{l}\text { Johnson, E. H } \\
\text { Sprague, J. J }\end{array}$ & $\begin{array}{l}\text { July } 8,1890 \\
\text { Sept. } 30,1890\end{array}$ & 450,744 & Johnson, E. H _....... & Apr. 21, 1891 \\
\hline
\end{tabular}

74. MACHINE ELEMENTS-13. Machine BRAKES.

452,619 Shaw, A.J

74. MACHINE ELEMENTS.-14. Mechanical Movenexts.

\begin{tabular}{|c|c|c|c|c|c|c|}
\hline $\begin{array}{l}444,284 \\
444,285\end{array}$ & $\begin{array}{l}\text { Warth, A } 1 \text { - } \\
\text { Warth, A }\end{array}$ & $\begin{array}{ll}\text { Jan. } & 6,1891 \\
\text { Jan. } & 6,1891\end{array}$ & $\begin{array}{l}444,286 \\
444,287\end{array}$ & $\begin{array}{l}\text { Warth, A } \\
\text { Warth, } \mathrm{A}\end{array}$ & $\begin{array}{l}\text { Jan. } \\
\text { Jan. }\end{array}$ & $\begin{array}{l}6,1891 \\
6,1891\end{array}$ \\
\hline
\end{tabular}

440,173 Macdonald, A. J

Nov. 11, 1890

75. METALLURGT.-34. Amalganators-Electric.

455,164 Button, G. and Wyeth, W. E June 30, 1891

75. NETALLURGT.-15. LEAD.

438,117 Shapleigh, Wr

440,268

440,269

440,270

440,271

Payen, C

Oct. $\quad 7,1890$

Nor. 11, 1890

Кัo. 11,1890

Payen, C

Nov. 11, 1890

Payen, C

Nov. 11,1890

Nov. 11,1890

440,273
440,274
440,275
440,276
440,277
440,775

I'ayen, C

Payen, C

Payen, C

Payen, C

Payen, C

Payen, C

Nov. 11,1890

Nov. 11, 1890

Nov. 11, 1890

Nov. 11, 1890

Nov. 11,1890

Nov. 11, 1890

75. METALLLRGY.-17. RedUCing AND SEPARATING.

452,030 Castner, H. Y

75. Metallikgl.-68. Solltion aśd Precipitatios-Electrolysis.

442,333 Roberts, I. L Iec. 9, 1s90 442,661 Bottome, T. D

Dec. 16,1890

78. HARDWARE MAKING.-5. Forgixg AND WELDING.

441,276 Bayles, J. C พัov. 25,1890

83. MILLS.-59. SePARATORS-WASHERS.

433,983 Hatch, D. P. and Guion, P. II Aug. 12, 1890

84. MLSIC.-81. Electric ACtions.

444,169 Fuller, L. K-

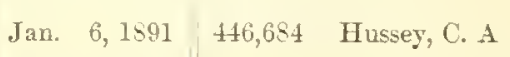
Feb. 17, 1891 84. MUSIC.-20. ORgax Bellows.

404,375 Smith, F. S 
88. OPTICS.-19. COMPASEE.

\begin{tabular}{|c|c|c|c|c|c|}
\hline No. & NAME. & DATE. & No. & NAME. & DATE. \\
\hline 437,169 & Aramburn, F - & Sept. 23,1890 & 441,579 & $\sigma^{\prime}$ Neil, $J_{---}$ & Nov. 25,1890 \\
\hline \multicolumn{6}{|c|}{ 88. OPTICS.-21. Distanee Instruments. } \\
\hline $\begin{array}{l}437,069 \\
441,972 \\
441,973\end{array}$ & $\begin{array}{l}\text { Wiesebrock, F. W } \\
\text { Rapieff, J } \\
\text { Rapieff, J }\end{array}$ & $\begin{array}{l}\text { Sept. } 23,1890 \\
\text { Dec. } 2,1890 \\
\text { Dec. } 2,1890\end{array}$ & $\begin{array}{r}441,974 \\
444,217\end{array}$ & $\begin{array}{l}\text { Rapieff, J } \\
\text { Fiske, B. A }\end{array}$ & $\begin{array}{ll}\text { Dec. } & 2,1890 \\
\text { Jan. } & 6,1891\end{array}$ \\
\hline
\end{tabular}

91. PAINTING.-11. Panting Machines.

443,237 Heppell, R. W Dec. 23,1890

92. PAPER MAKING.-7. FABRICS.

442,335 Roberts, I. L

Dec. 9,1890

92. PAPER MAKING.-5. Digesters.

$440, \neg 42$ Wagg, S. R

Nov. 11,1890

93. PaPer Mandfacteres.-21. Perforatixg Lace Paper.

$\pm 31,280$ Odell, J. B July 1,1890

95. PHOTOGRAPHY.-5. CAMERAS.

432,783 Blomgren, W. A

95. PHOTOGRAPHY.-30. CoMBINED APPARATUS.

441,158 Harbeck, W. H

96. COATING WITH METAL.-1. ELectro-Deposition.

\begin{tabular}{|c|c|c|c|c|c|}
\hline $\begin{array}{l}436,895 \\
441,892 \\
41,893\end{array}$ & $\begin{array}{l}\text { Jeançon, J. A } \\
\text { Possons, W. J } \\
\text { Possons, W. J. }\end{array}$ & $\begin{array}{l}\text { Sept. } 23,1890 \\
\text { Dec. } 2,1890 \\
\text { Dec. } 2,1890\end{array}$ & $\begin{array}{l}441,894 \\
449,890 \\
453,355\end{array}$ & $\begin{array}{l}\text { Possons, W. J } \\
\text { Zingsen, F. W } \\
\text { Haswell, A. E. and A. G }\end{array}$ & $\begin{array}{l}\text { Dec. } 2,1890 \\
\text { Apr. } 7,1891 \\
\text { June } 2,1891\end{array}$ \\
\hline
\end{tabular}

98. PNEUMATICS.-27. FANS-AUTOMatic.

4501,873 La Boiteaux, F. M

May 5, 1891

101. PRINTING.-5. Branding 'Stamps.

\begin{tabular}{l|l}
\hline $45,648 \mid$ Cannell, W $W \ldots \ldots \ldots \ldots \ldots \ldots \ldots$ & \\
\hline
\end{tabular}

101. PRINTING.-20. Hand Stamps.

4.34,396 Mallonee, J. D

441,430 Mallonee, J. D

445,161

Chase, $\mathrm{H} . \mathrm{A}$.
Aug. 12, 1890 Noจ. 25,1890
Jan. 27, 1891
Martindale, W. B. and Malm- Mar. 24, 1891 borg, E. R.

Martindale, W. B 
101. PRinting. - $t$ t. Matrix Making.

\begin{tabular}{|c|c|c|c|c|c|}
\hline No. & NAME. & DATE. & No. & NAME. & DATE. \\
\hline $\begin{array}{l}435,338 \\
436,703 \\
447,134 \\
4 t 7,135\end{array}$ & $\begin{array}{l}\text { Watson, J. A } \\
\text { Goodson, G. A } \\
\text { Lee, H. and Lebrun, E. } \\
\text { Lee, H }\end{array}$ & $\begin{array}{l}\text { Aug. } 26,1890 \\
\text { Sept. } 16,1890 \\
\text { Feb. } 24,1891 \\
\text { Feb. } 24,1391\end{array}$ & $\begin{array}{l}+17,321 \\
147,419 \\
+48,587 \\
448,994\end{array}$ & $\begin{array}{l}\text { Hargrave, T. C } \\
\text { Hargrave, T. C } \\
\text { Redfield, C. L } \\
\text { Redfield, C. L }\end{array}$ & $\begin{array}{l}\text { Mar. 3, } 1891 \\
\text { Mar. 3, } 1891 \\
\text { Mar. 17, } 1891 \\
\text { Mar. 24, } 1891\end{array}$ \\
\hline
\end{tabular}

101. PRINTING.-36. Stereotype Plate Holders.

443,278 Hanson, E. H Dec. 23,1890

101. PRINTIN r. 12. TrPe-IVRTrig.

$\$ 36,319$ Silkman, E. J

4 t5, 271 Silkman, E. J

\section{Sept. $9,1890 \quad 449.923$ Youngs, E. F}

Nar. $1 \overline{7}, 1891$ 453,430 IIcLaughlin, J. F
Apr. 7,1891

June 2, 1591

\section{PRosECTILEs.-9. MLana Torpedoes.}

$431,50.5$ Donnelly, A. G.

July 8,1890

102. PROJECTILER,-11. Prianers.

436,023 smith, H. J

Sept. 9,1890

103. PLMPS-13. DOtBLE ['ISTOA.

439,792 storey, I. E

$452, \div 01$, Thatcher, E. H

104. R.ALMA IN-9.9. GRIPPERS - WhELP WHEEL.

450,159 Pocock, F. 1 Apr. 1t, 1591

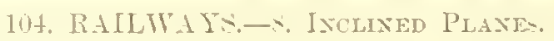

432.615 Henuing, B. F July 22,1890

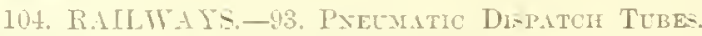

431.698 Leaks, S. F

t31.699 Leaks, $\therefore$ F

July $\rightarrow, 1540$ 431.7(0) Leaks, S. F Jul: 1 ty t31,701 Leals, S. F
July 8,1890 July s, 1890

104. RAILWA IS-TI, SIGXALS-DAXGER.

$\pm 13,730$ steele, J. IT Dee. 30,1890

104. RAILWAYS-74. SIGALS-TRIS.

443,131 Steele, J. W

104. RALTYA IS-24. SWTTCHE-TRA3WAY. 
104. RAILWAYS.-34. Track Cleaners-Wheer Fenders.

\begin{tabular}{|c|c|c|c|c|c|}
\hline No. & NAXE. & DATE. & No. & NAME, & DATE. \\
\hline 443,738 & Appleyard, A. E & Des. 30,1890 & $450, \pm 60$ & Hines, $\mathrm{D}$ & Apr. 14, 1891 \\
\hline \multicolumn{6}{|c|}{ 105. RAILWAY CARS.-6. BRAKES. } \\
\hline $\begin{array}{l}131,063 \\
416,935\end{array}$ & $\begin{array}{l}\text { Illingworth, J. and Baker, A. II } \\
\text { Smith, R. T - }\end{array}$ & $\begin{array}{l}\text { Aug. } 12,1890 \\
\text { Feb. } 24,1891\end{array}$ & 451,450 & Bassett, N- & June 23,1891 \\
\hline \multicolumn{6}{|c|}{ 105. RAILWA T CARs.-28. Steani Street. } \\
\hline $\begin{array}{l}433,754 \\
434,097\end{array}$ & $\begin{array}{l}\text { Wharton, W., jr } \\
\text { Sperry, E. A. }\end{array}$ & $\begin{array}{l}\text { Aug. } 5,1890 \\
\text { Aug. } 12,1890\end{array}$ & 434,098 & Sperry, E. d--- & Aug. 12, 1890 \\
\hline
\end{tabular}

112. SEMING MACHINES-24. SPECIAL MaCHINES.

454,404 Ames, $\mathrm{F}$

114. SHIPS.-10. Subrarine hind Torpedo.

453,s61 Halpine, N.J

June 9,1891

116. SIGYALS. - 1. ALARMS.

441,982 Simpson, I. H

Dec. 2, 1890

116. SIGNALS-11. AlARMS-Frre.

434,572 Iske, A. and A

116. SIGNALS.-33. BeLdS.

436, 560 Doyle, G-

116. SIGNALS.-35. DOOR BELLS.

433,724 Emme, C. A

116. SIGNALS.-27. STATION INDICATORS.

439,015 Kirwan, G. H.

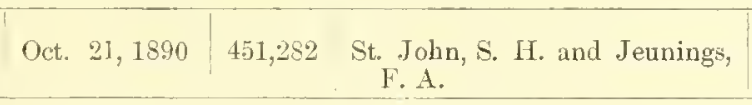

Apr. 28, 1891 F. A.

120. STATIONERY.-26. Post OFFice Boxes.

455,126 Smith, W. A. M. and R. T. F

June 30, 1891

122. STEAM BOILERS.-11. INDICATORS.

433,872 Hoore, C. W

Aug. 5, 1590

123. AIP AND GAS ENGINES.-3. ExplOsIve.

436,936 Eisenhuth, J. W

439,200 Shanck, H. K.

$439,29,2$ Baldwin, C. WT

\section{Sept. 23, $1890 \quad 448,369$ Regan, D. S}

\begin{tabular}{lll|l} 
Oct. 28, 1890 & 454,238 & Tremper, F. E. aud Eisenhuth, June 16,1891
\end{tabular} 
125. STONE WORKING.-10. DRILIS.

\begin{tabular}{|c|c|c|c|c|c|}
\hline No. & NAME. & DATE. & No. & NaME. & Date. \\
\hline 445,102 & Van Depoele, C. J & Jan. 20,1891 & 449,090 & Schlesinger, W. M & Mar. 24, 1891 \\
\hline
\end{tabular}

125. STONE WORKING.-14. Mining Machines.

\begin{tabular}{l|l|l|l|l|l}
\hline $.431,239$ & Walter, J. MI _.......... July 1, 1890 & 451,942 & Lechner, F. M _........... May 12, 1891 \\
\hline
\end{tabular}

126. STOVES AND FURNACES.-14. Dampers-AUtomatic.

441,591. Snyder, J. M

441,745 Butz, A. M

126. STOVES AND FURNACES.-112. Dampers-Automatic-Pregsure Motor.

466,212 Shorey, G. L

Feb. 10,1891

126. STOVES AND FURNACES.-51. Shovels, Tongs, and Pokers.

433,284 Gould, W. P

126. STOVEs AND FURNACES.-61. Stove Pipe Thimbles.

441,644 Conderman, G. IV

134. METAL TUbixg AND WIRE-2, Lead Pipe Making.

\begin{tabular}{l|l|l|l|l|l}
\hline 443,551 & Stillman, F. H.............. & Dec. 30, $1890 \quad 451,960$ & Tracy, L. W............... & May 12, 1891 \\
\hline
\end{tabular} 134. METAL TLBING AND WIRE.-6. TUBES.

441,277 Bayles, J. C

134. METAL TLBING AND WIRE-16. Wire.

443,536 Norman, T. IV \begin{tabular}{l|l|l} 
Dec. $30,1890,448,594$ & Smith, C. R
\end{tabular} Mar. 17, 1891

136. STEAM ENGINE TALVES.-11. Throtthe.

439,445 Tushingham, F. G. and Iubois, Oct. 28, $1890 \quad 442,956 \quad$ Conroy, T. and Fuhrman, L. P. Dec. 16, 1890 H.

137. WATER DISTRIBUTION,-20. SiPHONS.

431,542 Bailey, G. WV July 8,1890

139. WEATING.-52. StopPING.

432,512 Coughlin, D. E July 22, 1890

140. WIRE WORKING.-24. MachINES FOR WINDING. 


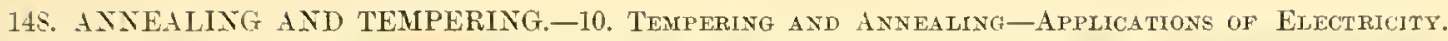

\begin{tabular}{|c|c|c|c|c|c|}
\hline No. & NAME. & DATE. & No. & NAME. & Date. \\
\hline $\begin{array}{l}448,464 \\
453,162\end{array}$ & $\begin{array}{l}\text { Sedgwick, F } \\
\text { Ries, E. E-... }\end{array}$ & $\begin{array}{l}\text { Dee. } 23,1890 \\
\text { May } 26,1891\end{array}$ & 453,163 & Ries, E. E & $\operatorname{May} 26,1891$ \\
\hline
\end{tabular}

149. CHEMICAL TREATMENT OF HIDES, SKINS, AYD LEATHER.-10. Tanning Processes.

$442,11 \overline{5}$ Groth, L. A

Dec. 9,1890

150. PACKING AND STORING VESSELS.-44. Glass and Potrery-Jars.

438,312 Enholm, O. A

154. COMPOSITE ROOFINGS AND COVERINGS.-4. Boller Coverings.

\begin{tabular}{|c|c|c|c|c|c|}
\hline $\begin{array}{l}433,471 \\
433,472 \\
433,473\end{array}$ & $\begin{array}{l}\text { Johns, H. WI } \\
\text { Johns, H. W } \\
\text { Johns, H. W }\end{array}$ & $\begin{array}{l}\text { Aug. } 5,1890 \\
\text { Aug. } 5,1890 \\
\text { Aug. } 5,1890\end{array}$ & $\begin{array}{l}441,165 \\
445,358\end{array}$ & $\begin{array}{l}\text { Johns, H. W } \\
\text { Sprinkmann, F }\end{array}$ & $\begin{array}{l}\text { Nov. } 25,1890 \\
\text { Jan. } 27,1891\end{array}$ \\
\hline
\end{tabular}

164. PLNCHING AND SHEARING.-3. SATPTG METAL.

\begin{tabular}{c|c|c|}
\hline 431,564 & Denison, S. P & July $\delta, 1890$ \\
\hline 166. ARTESIAX AND OIL WELLS.3. DRILLIXG AND BoriNG.
\end{tabular}

166. ARTESIAN AND OIL WELLS.3. DRILLIXG AND BorING.

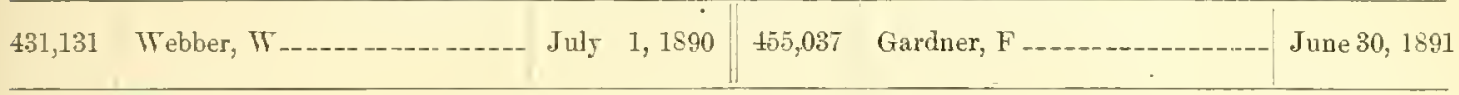

181. ACOUSTiCS.-2. Ear Trumpets.

418,627 Benjamin, G. V.

181. ACOUSTICS.-3. Mechanical Telephones.

442,799 Carr, R. S -

184. LUBRICATORS.-8. MISCELLANEOUS.

432,927 Barriett, S. L

186. STORE SERVICE.-1. GRAVITY.

439,726 Elliott, G. R

Nov. 4,1890

186. STORE SERVICE.-7. SwITCHEs.

\begin{tabular}{l|l}
\hline $448,15 i$ & Moyer, J. W. and Jackson, G. F.
\end{tabular}

186. STORE SERVICE.-9. VENDING APparatus.

\begin{tabular}{|c|c|c|c|c|c|}
\hline 434,109 & Harris, A - & Aug. 12, 1890 & 438,174 & Patterson, J. Y & Oct. 14,1890 \\
\hline 434,395 & Little, R. H & Aug. 12, 1890 & 439,477 & Coffin, C. I & Oct. 28,1890 \\
\hline 437,293 & 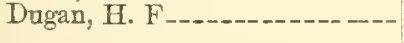 & Sept. 30, 1890 & $439, \pm 78$ & Coffin, C. L - & Oct. 28,1890 \\
\hline
\end{tabular}

187. ELEVATORS.-1. Elevators.

446,907 Whitlock, E

\begin{tabular}{|l|l|l} 
Feb. 24, 1891 & 447,899 & Otis, N. P
\end{tabular}

Mar. 10, 1891 
188. FLUID PRESSURE RAILWAY BRAKES.-4. Electric.

\begin{tabular}{|c|c|c|c|c|c|}
\hline No. & Name. & DAte. & No. & Name. & Date. \\
\hline 452,942 & Slater, W. W. . & & & & May 26,1891 \\
\hline
\end{tabular}

192. CLUTCHEs.-15. Ixtermittent Grip.

437,428 Edison, T. A

194. ColN CONTrolled aPParatUs.-5. Article Delivery-Slydabe Pusher, Coin Exgaged. 441,259 Sylvester, P. L

Nov. 25,1890

194. COIN CONTROLLED APPARATUS.-10. EXHIBITORS-SURFACE.

455,100 Harris, $\mathrm{A}$

June 30,1891

194. COIN CONTROLLED APPARATUS.-11. Fluid Delrerr.

441,861 Van Sant, IT. S. and Eames, E. C.

Dec. 2,1890

194. COIN CONTROLLED APPARATUS.14. InduCtion CoILs.

$445,220 \mid$ Colby, E. J

Jan. 27,1891

194. COIN CONTROLLED APPARATUS.16. NisCELLANEOUS.

\begin{tabular}{l|l|l|l|l|l}
\hline 448,021 & Gray, W .. _..._.......... & Nar. 10, 1891 & 452,068 & Ashwell, W. H. ............. & May 12, 1891
\end{tabular}

194. COIA CONTROLleI APPARatUd-17. Phosographs.

194. COIN CONTROLLED APPARATUS.23. WEIGHING.

454,529 Rutter, G. F

June 23, 1891

200. TOWERS-3. Telegraph Poles.

431,955 schneider, J. F.and Carson, D.K July 8, $1890 \quad 449,977$ Stern, W. A

449,753 Brownlee, J. N-........ Apr. $7,1891 \quad 452,255$

Fletcher, J.

449,777

452,255

Iilliken,

Bray, T. J

Apr. 7, 189

Hall, O. B

Apr. 7, 1391

Lieb, C.

May 5, 1891

Iay 5, 1891

May 12,1891

June 2, 1891 


\title{
APPENDIX No. X.
}

\author{
PART II.
}

CONSISTING OF A

\section{NUMERICAL AND CHRONOLOGICAL INDEX OF ALL PATENTS}

RELATING TO

\section{포폴エ○エエY}

Issued from July 1, 1890. to June 30, 1891, inchusive, giving names of Inventors, - numbers and dates of Patents, and the sub-class to which each belongs. 

OF

\section{Patents Relating to Electricity}

From July 1, 1890 , to June 30, 1891.

\begin{tabular}{|c|c|c|c|c|}
\hline No. & & ClaAss. & \multicolumn{2}{|c|}{ DATE. } \\
\hline 035 & af & Cut-outs, electro-magnet & July & 1,1890 \\
\hline & & Lights, incandescent, spe & July & 1,1890 \\
\hline & Il, J. & Cut-outs, thermal & July & 1,1890 \\
\hline & h, M. V & Batteries, galvanic & July & I, 1890 \\
\hline & & Telephones, calls--- & July & 1,1890 \\
\hline & heeler, $F_{-}$ & Railways, systems, overhead _ & July & 1,1590 \\
\hline & er, $\mathrm{F}$. & - do -1 & July & 1,1890 \\
\hline & $T$ & dr & July & 1,1890 \\
\hline & er, $F$ & do $-\ldots$ & July & ], 1890 \\
\hline & & Insula & July & 1,1890 \\
\hline & O. $\mathrm{F}$ & nical _ _-_. & July & 1,1890 \\
\hline & W & g and boring --.---. & July & $1,1890^{\circ}$ \\
\hline & & lectric_._-_... & July & 1, 1890 \\
\hline & $\mathrm{F}$ & liy, fire -------- & July & 1,1890 \\
\hline & H. & $n$ of power & July & 1,1890 \\
\hline & P. B & -n- & July & 1,1890 \\
\hline & L. B & Cut-outs, thermal ...---- & July & 1, 1890 \\
\hline & L. B & ------do - - - - - - - & July & 1, 1890 \\
\hline & fall, & tives_._-_- & July & 1,1890 \\
\hline & T. & Iternating - --_- & July & 1,1890 \\
\hline & & -- & July & 1,1890 \\
\hline & WI. & - & July & 1,1890 \\
\hline & berge & $------d o----$ & July & 1,1890 \\
\hline & J. & Mining mach & July & 1,1890 \\
\hline & $\mathrm{J}$ & ------ & July & 1,1890 \\
\hline & F. E & ives & July & 1,1890 \\
\hline 1 & קחק & --------- & July & 1,1890 \\
\hline & & & July & 1,1890 \\
\hline & & pecial & July & \\
\hline & 1 & ndary & July & 1,1590 \\
\hline & & ls ----- & July & 1,1890 \\
\hline & & & July & 590 \\
\hline & $F$ & ports, and in & July & \\
\hline & $\mathrm{L}$ & -....... & July & 1, \\
\hline & & Arv & July & 1, 1890 \\
\hline & 1. & s & July & 1,1890 \\
\hline & & vel & July & $\overrightarrow{1}$, \\
\hline & poel & cating --.--- & July & 1,1890 \\
\hline & & Ho & July & 1,1890 \\
\hline & & Tra & July & 1,1890 \\
\hline & & & July & \\
\hline & $T$ & 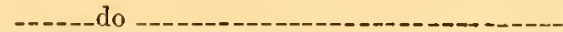 & July & $\overrightarrow{1}$ \\
\hline & ind Curry, J. H. & ienl & July & 1,1590 \\
\hline & $\mathrm{MT}$ & I & July & 90 \\
\hline & & & July & 1, 1890 \\
\hline & & & July & 8,1890 \\
\hline & & L & July & S, 1890 \\
\hline & & ric, hand machines -. - & July & 8,1890 \\
\hline & & & July & S, 1890 \\
\hline & & & July & 8,1890 \\
\hline & & aine & July & S, 1890 \\
\hline & & & July & \\
\hline & & & July & 8,1590 \\
\hline & & iscellan & July & \\
\hline & & & July & 1590 \\
\hline & & Sto & July & 8,1890 \\
\hline & & systems, conduit & July & 8,1890 \\
\hline & J. H_- & ----- & July & 8,1890 \\
\hline & & & July & \\
\hline & & & July & 8,18 \\
\hline & $y$, J. H. & -----1 & July & 8,1890 \\
\hline & ( & L & July & \\
\hline & & Railways, systems, overhead, trolleys & July & 8,1890 \\
\hline
\end{tabular}




\begin{tabular}{|c|c|c|c|}
\hline No. & Е. & Cluass. & Date. \\
\hline & & Railways, pneumatic dispatch tu & July 8,1890 \\
\hline & Leaks, S. F- & _. do & July 8,1890 \\
\hline & Leaks, S. F- & -_...-do - --- & July 8,1890 \\
\hline & Leaks, S. F.. & 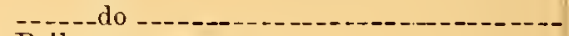 & July $\quad 8,1890$ \\
\hline 711 & Short, S. H & Railways, systems -- & July 8,1890 \\
\hline & L. B & Cut-outs, thermal -.-. & July 8,1890 \\
\hline & , R. M & Electric loeomotives & July 8,1890 \\
\hline & S. J & Lights, incandescent, special feat & July 8,1890 \\
\hline & , C. I & Batteries, galvanie & July 8,1890 \\
\hline 76 & $\mathrm{R}$ & Lights, incandescent & July 8,1890 \\
\hline & D. a & Applications, general ... & July 8,1890 \\
\hline & W. L & Conduetors, underground & July $\quad 8,1890$ \\
\hline 784 & . $\mathrm{B}--$ & Telegraphy, printing & July 8,1890 \\
\hline & & Telegraphy, automatic --- & July 8,1890 \\
\hline & $\mathrm{F}$ & - do & July $\quad 8,1890$ \\
\hline 10 & $\mathrm{C}$ & Telephones, supports _... & July 8,1890 \\
\hline & E. H & ne elements, gearin & July 8,1890 \\
\hline 12 & E. H & Regulators _....... & July 8,1890 \\
\hline 823 & . $\mathbf{J}$ & comotives ...... & July 8,1890 \\
\hline 879 & $\mathrm{a}, \mathrm{E}$ & Systems of distribution & Tuly 8,1890 \\
\hline 883 & ass, L. F & Phonographs _._-_- & July 8,1890 \\
\hline & & Alar & July 8,1890 \\
\hline & D. K.... & , telegraph poles.- & July 8,1890 \\
\hline 32 & E. II - & nes, & July 8,1890 \\
\hline & C. 1 & Batt & July 8,1890 \\
\hline 73 & T_- & Con & July 8,1890 \\
\hline 77 & M & $\mathrm{I}$ & July 8,1890 \\
\hline 78 & A. W & $\mathrm{cal}$ & July 8,1890 \\
\hline & I. $\mathrm{E}-$ & Teleg & July 8,1890 \\
\hline & $\mathbf{T}$ & hy, printing -. & July 8,1890 \\
\hline & WV. & cutting & July 8,1890 \\
\hline & ( & $\mathrm{R}$ & July 8,1890 \\
\hline & & Ind & July 15,1890 \\
\hline & & $\mathrm{Co}$ & July 15,1890 \\
\hline & MI. & $\mathrm{R}$ & July 15,1890 \\
\hline 50 & C. & foriums, transformers, stationary & July 15,1890 \\
\hline & & 8 & July 15,1890 \\
\hline 33 & & , orerhe & July 15,1890 \\
\hline & & Rail & July 15,1890 \\
\hline & MI & y', relays and so & July 15,1890 \\
\hline 31 & 1 & ts..--1. & July 15,1890 \\
\hline 36 & & loeomotives...-_ & July 15,1890 \\
\hline 142 & W. II & 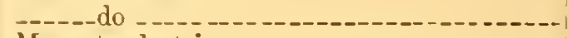 & July 15,1890 \\
\hline 169 & & Magneto-electric -... & July 15,1890 \\
\hline 97 & & I & July 15,1890 \\
\hline 02 & & $\mathrm{~B}$ & July 15,1890 \\
\hline 04 & ( & us, eonduit - & July 15 \\
\hline & & & July 10,1890 \\
\hline 06 & & Mag & July 15,1890 \\
\hline 77 & & $\mathrm{C}$ & July 15,1890 \\
\hline $0 \mathrm{~s}$ & & loe & July 15,1890 \\
\hline & & nes --. & July 15,1890 \\
\hline & & $\mathrm{I}$ & July 15,1890 \\
\hline 234 & & , mechanieal .. & July 15,1890 \\
\hline 37 & & locomotives ..... & July 15,1890 \\
\hline 279 & 1 & & July 15, I 890 \\
\hline 284 & & Lights, arc, gravity feed, clu & July 15,1890 \\
\hline & & Conductors & July 15,1890 \\
\hline $34 \overline{5}$ & ele, & ric locomotives _-_ & July 15,1890 \\
\hline & & -eleetrie -..... & July 15,1890 \\
\hline 16 & & & July 10 \\
\hline 32 & P & Applications, tools and machines & July 15,1890 \\
\hline & & do -1 & July 15,1890 \\
\hline 53 & Tay & rinting - & July 15,1890 \\
\hline & & & July 15,1890 \\
\hline & & & July 10 \\
\hline & & Electric locomotives _- & July 15,1890 \\
\hline & & Stc & July 22, 1890 \\
\hline 521 & $\mathrm{Fi}$ & $1--$ & July 22,1890 \\
\hline & & Telephones, s & July 22, 1890 \\
\hline & & & July 22,1890 \\
\hline 571 & --- & Railways, systems, eonduit & July 22,1890 \\
\hline & -- & Magneto-electrie --- & July 22,1890 \\
\hline & Th & is, overhead & July 22,1890 \\
\hline & Hen & Railways, ineline planes .. & July 22, I\$990 \\
\hline $\begin{array}{l}452,618 \\
432,619\end{array}$ & $\begin{array}{l}\text { Holcombe, } \mathrm{A} \text {. } \\
\text { Holcombe, } \mathrm{A} \text {. }\end{array}$ & legraphy, fire -...-.... & $\begin{array}{l}\text { July } 22,1890 \\
\text { July } 22, \text { I } 890\end{array}$ \\
\hline & & & \\
\hline
\end{tabular}




\section{No.}

Nane.

432,6223

432,629

432,630

$432,6 \pm 1$

432,645

432,646

432,651

432,652

432,633

$432,6 \overline{5} 4$

432,605

432,656

432,657

432,670

432,672

432,673

432,674

432,675

432,681

432,694

432,707

432,710

432,724

432,727

432,745

432,748

432,752

432,753

432,783

432,823

432,826

432,831

432,883

432,881

432,894

432,896

432,927

432,932

432,944

432,949

432,965

432,968

432,973

432,978

432,979

432,980

432,988

433,022

433,025

433, 041

433,045

433,046

433,047

433,051

433,082

433,120

433,167

433,170

433,174

433,180

433,181

433, 187

433,210

433,215

433,216

433,229

433,254

433,269

433,284

433,307

433,359

433,360

433,371

432,375

433,381

433,384

433,390

Lemp, $\mathrm{H}$

Rice, E. W. jr

Richards, J. E

Smith, MI. H

Thomson, E-

Thomson, E

Thomson, E

Thomson, E

Thomson, E

Timmis, 1 .

Jennings, B

Kookogey, W. P

Mansfield, $\mathrm{F}$

Mansfield, F

Mansfield, F

Waite, H. F

Chappell, G.

Poland, L. X. P

Carr, C. P

Dewey, M. TV

Edgerton, $\mathrm{X} . \mathrm{H}$

Hunter, R. M

Hunter, R. MI

Blomgren, W. A

Finney, G. S

Goehst, J. H

Morris, H. G

Lebenberg, $\mathrm{S}$

Lehman, J. H

Porter, C. S

Barriett, S. L

Burton, W.
Jennings, $\mathrm{B}$

Lucas, A. H

Aldrich, F. H

Bayley, C. H

Chase, H. A

Chase, H. A

Chase, H. A

Noyle, C. B.

Patterson, W.

Saunderson, I

Scribner, C. E

Scribner, C. E

Spang, H. IT

Jones, F. W

Field, S. D

Glover, J. B

Hunter, R. M

Kookogey, W.

Parcelle, A. L

Parcelle, A. L

Shapleigh, M.

Rabinowicz

Reppy, A. W

Bernstein, $A$

Niles, H. B

Belt, P. P

Gould, W. P

Robinson, W. E

McLaughlin, J. F

Schimer, A.

Sparrow, F. M

Williams, J. T

Woodward, J. H
Belding, W. S.-
Hunter, R. M

Cooke, E. T. and Mackar, W. H

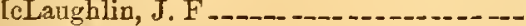

Class.

Date.

Electric locomotives

do

Electric locomotives

Recorders.-

Heaters

Magneto-electric

Heaters

Meters

Magneto-electric

Heaters

Batteries, secondary, applicati

Railways, systems, conduit

Batteries, secondary

Railways, systems, closed conduit _........... do

Locomotion

Batteries, galvanic

Indicators -

Motors

Lights, flaments and carbons

Telegraphy, circuits and systems -........

Heaters.

Lighting, systems .

Magneto-electric

Railways, systems, overhead, trolleys_-_-

Electric locc

Photography, cameras

Separators -

Lamp shades.

Batteries, secondary

Telephones, supports

Igniting devices, manua

Rheostats

Electro-magnets

Lubricators, miscellaneous

Heaters

Railways, systems, conduit

Lights, arc, special features

Lights, incandescent special features

Batteries, galvanic

Cut-outs, electro-magnetic--on-

Cut-outs, thermal

Motors --- - - -

Conductors, underground

Lights, filaments and carbons

Cut-outs, thermal -

Telephones, systems

Magneto-electric

Teleoraphy, circuits and syst

Telephones, electric

Indicators

Electric locomotives

Batteries, secondary

Electric locomotives

Cut-outs, thermal

Electric locomotives

Insulating materials

Telegraphy, railway-car

Cut-outs, thermal

Glass, plate and window

Regulators -.

Shovels, tongs, and pokers

Indicators

Cut-outs, mechanical

Railways, systems, battery

Lights, sockets and keys

Valve controllers

Railways, systems, telpher, solenoid.......

Electrodes

Magneto-electric

July 22,1890

July 22, 1890

July 22,1890

July 22,1890

July 22, 1890

July 22,1890

July 22,1890

July 22, 1890

July 22,1890

July 22,1890

July 22,1890

July 22,1890

Juiy 22,1890

July 22,1890

July 22,1890

July 22, 1890

July 22, 1890

July 22,1890

July 22, 1890

July 22, 1890

July 22,1890

July 22, 1890

July 22, 1890

July 22, 1890

July 22, 1890

July 22, 1890

July 22, 1890

July 22, 1890

July 22, 1890

July 22, 1890

July 22, 1890

July 22, 1890

July 22, 1890

July 22, 1890

July 22, 1890

July 22, 1890

July 22, 1890

July 22, 1890

July 22, 1890

July 22, 1890

July 29, 1890

July 29, 1890

July 29, 1890

July 29, 1890

July 29, 1890

July 29, 1890

July 29, 1890

July 29, 1890

July 29, 1890

July 29, 1890

July 29,1890

July 29, 1890

July 29, 1890

July 29, 1890

July 29, 1890

July 29, 1890

July 29, 1890

July 29, 1890

July 29, 1890

July 29, 1890

July 29,1890

July 29, 1890

July 29,1890

July 29, 1890

July 29, 1890

July 29,1890

July 29, 1890 


\begin{tabular}{|c|c|c|c|}
\hline No. & NaMe. & Cllass. & Date. \\
\hline & Felaing, w. s & Magneto-electric. & July 29,1890 \\
\hline & & - & July 29,1890 \\
\hline & Belding, W. S & $--d$ do -- & July 29,1890 \\
\hline & ote, T. M -- & Motors - - - & July 29,1890 \\
\hline & Foote, T. MI -- & Electric locomotives & July 29,1890 \\
\hline & Foote, T. M & 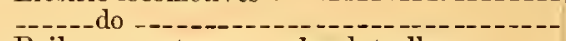 & July 29,1890 \\
\hline & Hunter, K. M & Railways, systems, overhead & July 29,1890 \\
\hline & Hunter, R. M & Electric locomotives & July 29,1890 \\
\hline & y, H. R & Railroads, systems, conduit & July 29,1890 \\
\hline & A. C - & Telegraphy, fire & July 29,1890 \\
\hline 24 & Scribner, C. E. & Telephones, systems & July 29, 1890 \\
\hline & e, F. J & , systems, overhead, trolleys & July 29,1890 \\
\hline & Wurts, $A$. and Baldwin, O. H & Telegraphy, lightning arresters & July 29,1890 \\
\hline & Clark, J. C _ . & (2) & Aug. 5,1890 \\
\hline & son, E. N & thermal. & Aug. 5,1890 \\
\hline & Ferrell, 1. M & ing rods ------ & Aug. 5,1890 \\
\hline & 1. W & Coverings --.-- & Aug. 5,1890 \\
\hline & W & -...--do & Aug. 5,1890 \\
\hline & H. W - & ------_do _- & Aug. 5,1890 \\
\hline & B. $\mathrm{J}_{--}$ & Telegraphy, fire & Aug. 5,1890 \\
\hline 86 & B. $\mathrm{J}_{--}$ & - do - & tug. 5,1890 \\
\hline & Noyes, B. J & --do - & Aug. 5,1890 \\
\hline 05 & J. $\mathrm{C}_{-}-$ & -_do - & Aug. 5,1890 \\
\hline & J. W --- & Magneto-electric & Aug. 5,1890 \\
\hline & A. $A$. & s, conduit & Aug. 5,1890 \\
\hline & L. L .. & - & Aug. 5,1890 \\
\hline & C. F & Jestris & Aug. 5,1890 \\
\hline 60 & W. 1 & 1,ett & Aug. 5,1890 \\
\hline 661 & L $=\ldots$ & 1nsulat & Aug. 5,1890 \\
\hline & C. $\mathrm{J}_{-}$ & ators & Aug. 5, 1890 \\
\hline 11 & I & tems, overhead, trolleys_-- & Aug. 5,1890 \\
\hline 19 & J. L L- & calls & Aug. 5,1890 \\
\hline & E. P & $\mathrm{Te}$ & Aug. 5, 1890 \\
\hline & I & Ga & Aug. 5,1890 \\
\hline & c. & $\mathrm{H}$ & Aug. 5,1890 \\
\hline & $\boldsymbol{J}$ & $\mathrm{Te}$ & Aug. 5,1890 \\
\hline & Robb, J. T & $\mathrm{Lis}$ & Aug. 5,1890 \\
\hline & T. G & d keys. & Ang. 5, 1890 \\
\hline & & ting & Aug. 5,1890 \\
\hline & & do & Aug. 5, 1890 \\
\hline & & ationary- & Aug. 5,1890 \\
\hline & & ing -..... & Aug. 5,1890 \\
\hline & P. and Bon & $\mathrm{Co}$ & Aug. 5,1890 \\
\hline & $\mathrm{E}$ & Do & Aug. 5,1890 \\
\hline & 1 & $\mathrm{~B}$ & Aug. 5,1890 \\
\hline & $1=0$ & $\mathrm{C}$ & Aug. 5,1890 \\
\hline & & $\ldots$ & Aug. 5,1890 \\
\hline & $\mathrm{Z}$ & & Aug. 5,1890 \\
\hline & J. G. L - & $\mathrm{Cl}$ & Aug. 5, 1890 \\
\hline & & ng - & Aug. 5,1890 \\
\hline & & & Aug. 5,1890 \\
\hline & & overhead, trolleys ... & Aug. 5,1890 \\
\hline & & , systems, battery - & Aug. 5,1890 \\
\hline & M & & Aug. 5,1890 \\
\hline & & $\mathrm{Cl}$ & Aug. 5,1890 \\
\hline & & $\operatorname{lr} r$ & Aug. 5,1890 \\
\hline & ad Hill & Rail ways, conductor supports and insulator & A ug. 5,1890 \\
\hline & & Electric locomotires & Aug. 5,1890 \\
\hline & encer, G. J & & Aug. 5,1890 \\
\hline & & L & Aug. 5,1890 \\
\hline & & ent, specia & Aug. 5,1890 \\
\hline & & Cor & Aug. 5,1890 \\
\hline & & s, circuits, multiple ar & Aug. 5,1890 \\
\hline & Wy & 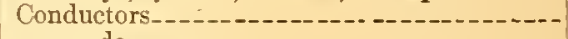 & Aug. 5,1890 \\
\hline & & do...--- & Aug. 5,1890 \\
\hline & II! & & Aug. 5,1890 \\
\hline & $\mathrm{Li}$ & & Aug. 12,1890 \\
\hline & $\mathrm{Pa}$ & & Aug. 12, 1890 \\
\hline & & ric --.---. & Aug. 12,1890 \\
\hline & $\mathrm{H}$ & ad coal washers -. & A ug. 12,1890 \\
\hline & & -- - - - - - & Aug. 12, 1890 \\
\hline & bbons, W. M & Elec & Aug. 12,1890 \\
\hline & $\stackrel{\mathrm{H}}{\mathrm{P}}$ & Rail & Aug. 12,1890 \\
\hline & $\stackrel{P}{S}$ & res & Aug. 12, \\
\hline & J. H & , conduit__-_._._. & $\begin{array}{l}\text { Aug. } 12,1890 \\
\text { Aug. } 12,1890\end{array}$ \\
\hline 4,038 & White, A. B _ & Drop lights and hangers & Aug. 12,1890 \\
\hline
\end{tabular}


So.

434,063

$434,0 \div 6$

434,087

434,093

434,096

434,097

434,098

434,109

434,133

434,144

$434,14 i$

434,145

434,149

434,151

434,152

434,153

434,154

434,159

434,162

434,163

$4334,16 \pm$

$43 \pm, 165$

434,166

$434,16-$

134,168

434,169

434170

434,175

434,180

434,181

434,186

434,199

424,206

434,224

434,261

434,275

434,276

434,301

434,363

434,371

434,376

434,38 2

434,389

434,390

434,391

434,395

431,396

434,410

434,427

434,428

434,429

434,444

434,450

434,457

434,458

431,468

431,488

434,489

434,500

434,501

434,509

$434, \overline{0} 30$

434,531

431,532

$434,5.57$

434,558

434,579

$434, \overline{5} 80$

434,581

434,582

434,584

434,585

434,586

434,587

434.589

434,590
Naye.

Cluass.

Date.

Street cars, brakes

Aug. 12, 1890

Batteries, secondary

Motors

Street cars, steam -1-2 -

Yending apparatus

Heater

Insulators_-_._.

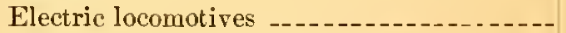

Lights, incandescent, special features

Aug. 12, 1890

Cut-outs, mechanical _.................. Aug. 12, 1890

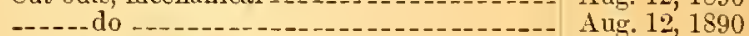

Lights, sockets and keys .................... 12, 1890

Galranometers -................... Aug. 12, 1890

Lights, incandescent

Systems of distribution -..._..._._._._._. Aug. 12, 1890

Telegraphy, lightning arresters --..-.-.--- Aug. 12, 1890

Inductoriums, transformers, stationary -...- Aug. 12, 1890

Conductors, underground _._........... Aug. 12, 1890

Telegraphy, lightning arresters ......... Aug. 12, 1890

- Aug. 12, 1590

Cut-outs, thermal _._. Aug. 12, 1890

-

Allg. 12, 1890

Lights, arc, gravity feed, clutch ............ Aug. 12, 1890

Motors _._._._._._. Aug. 12, 1890

Testing machines -.. _..._._..._._._. Aug. 12, 1890

Cut-outs, thermal _.................. Aug. 12, 1890

Batteries, secondary -........................ 12, 1890

Magneto-electric _..._-_._._...... Aug. 12, 1890

Batteries, secondary _......................... Aug. 12, 1890

Telegraphy, printing._-_._-_._-_._-_._- Aug. 12, 1890

Railways, systems, circuits, multiple are --_- Ang. 12, 1890

Railways, systems, overhead, trolleys _...-.- Aug. 12, 1890

Batteries, secondary_-_._._._._._... Aug. 12, 1890

Electric locomotives -._-_-_._-_._._...- Aug. 12, 1890

Telegraphy, lightning arresters........ Aug. 12, 1890

Batteries, secondary

Applications, tools and machines.......... Aug. 12, 1890

Railways, systems, conduit ..........-... Aug. 12, 1890

Aug. 12, 1890

Electric locomotives .................... Aug. 12, 1890

Vending apparatus _..................... Ang. 12, 1890

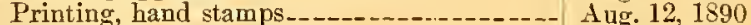

Railways, systems, closed conduit -...-_- Ang. 12, 1890

Batteries, thermal ........................ 19, 1890

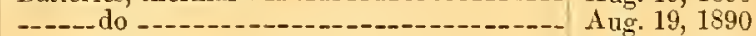

-_._. do -

Heaters - -

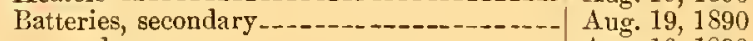

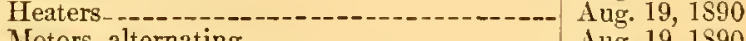

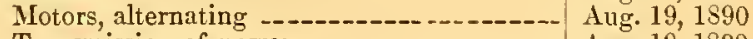

Transmission of power _._._._. Aug. 19, 1890

Batteries, thermal

Meters -

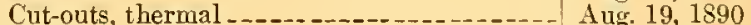

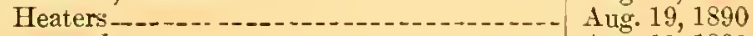

. Aug. 19, 1890

Galvanometers -....- Aug. 19, 1890

-. do -...... Aug. 19, 1890

Railways, systems, battery -_.___._._._._. A ug. 19, 1890

-_._do _-_._._. Aug. 19, 1890

-

--_do -

Registers -..-. Aug. 19, 1890

Telegraphy, relays and sounders _......... Aug. 19, 1890

Pyro-magneto electric devices _._._-_-_.- Aug. 19, 1890

Batteries, thermal .... Aug. 19, 1890

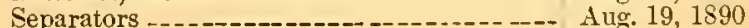

Electric locomotives _..._.______._._._. Ang. 19, 1890

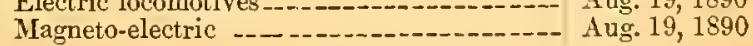




\begin{tabular}{|c|c|c|c|}
\hline No. & IE. & Class. & Date. \\
\hline & Fink, IV. . & Batteries, galvani & Aug. 19, 1890 \\
\hline & Hathaway, & Telegraphy, circuit & Aug. 19,1890 \\
\hline & Walter, I. E & Systems of distribution ..... & Aug. 19,1890 \\
\hline & Maxwell, D. & Bridges, towers & Aug. 19,1890 \\
\hline 1,640 & Mestern, $\mathrm{H}$ & Batteries, thermal & Aug. 19,1890 \\
\hline 671 & Iellett, L- & Applications, tools and machines -- & Aug. 19,1890 \\
\hline 34,682 & nd Wescott, E.J & Railways, systems, overhead, trolleys & Aug. 19,1890 \\
\hline & $J_{-} \ldots \ldots$ & Railways, systems, converter & Aug. 19,1890 \\
\hline 4,685 & Van Depoe & - & Aug. 19,1890 \\
\hline 34,686 & Tan Depoel & $\ldots \ldots$...... & Aug. 19,1590 \\
\hline & J & -do -...-..- & Aug. 19,1890 \\
\hline 4,746 & 年 & Medical and surgical, body-wear & Aug. 19,1890 \\
\hline 774 & & -...... & Aug. 19,1890 \\
\hline & $J_{-\ldots \ldots n}$ & & Aug. 19,1890 \\
\hline 4,827 & Gorton, A - & systems, closed conduit ... & Aug. 19,1890 \\
\hline & $\ldots$ & $y$, circuits and syst & Aug. 19,1890 \\
\hline & F. A & Rheostats & Aug. 19,1890 \\
\hline 63 & Cornir & Railways, systems, battery -- & Aug. 19,1890 \\
\hline & ........ & Bat & Aug. 19,1890 \\
\hline & ----1 & pher ......... & Aug. 19,1890 \\
\hline & $-\ldots-$ & ( & Aug. 19,1890 \\
\hline 434,879 & $\mathrm{O}$ & & Aug. 19,1890 \\
\hline 434,880 & -.....- & aphy, printing & Aug. 19,1890 \\
\hline 81 & --...-.- & do & Aug. 19,1890 \\
\hline & $-\ldots$ & ric locomotive & Aug. 19,1890 \\
\hline 34,885 & ........ & $r_{0}$ & Aug. 19,1890 \\
\hline & the- & & Aug. 19,1890 \\
\hline 99 & II & ical ........... & Aug. 19,1890 \\
\hline 17 & & lescent $----1---1-1$ & Aug. 26,1890 \\
\hline 434 & D & $\mathrm{Te}$ & Aug. 26,1590 \\
\hline & ....... & candescent, special features & Aug. 26,1890 \\
\hline & .......-. & - & Aug. 26,1890 \\
\hline & $\mathrm{P}$ & comotives _..... & Aug. 26,1890 \\
\hline 51 & $\ldots$ & prs n- & Aug. 26,1590 \\
\hline 61 & $\mathrm{~T}$ & ductor supports and inst & Aug. 26, 1890 \\
\hline & $\ldots$ & - & Aug. 26, 1890 \\
\hline & $-\cdots$ & 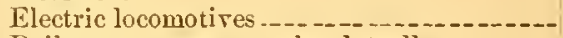 & Aug. 26,1890 \\
\hline & 急 & overhead, trolleys & Aug. 26,1890 \\
\hline 015 & -..-- & 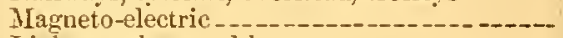 & Aug. 26,1590 \\
\hline 24 & $\mathrm{R}$ & sockets and keys & Aug. 26, 1590 \\
\hline 029 & Bren & do - - & Aug. 26,1890 \\
\hline 030 & B & $\mathrm{Cl}$ & Aug. 26, 1890 \\
\hline $4 \overline{7}$ & S & d keys ... & Aug. 26,1890 \\
\hline & -.--.- & & Aug. 26,1590 \\
\hline &.--- & eys -- & Aug. 26, 1890 \\
\hline & & applications & Aug. 26,1890 \\
\hline 43 & 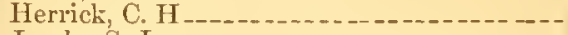 & - & Ang. 26, 1890 \\
\hline 97 & & $\mathrm{Re}$ & Aug. 26,1890 \\
\hline & ...... & 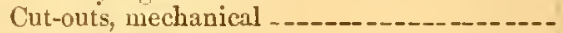 & Aug. 26, 1590 \\
\hline & - & $\mathrm{Cl}$ & Aug. 26,1590 \\
\hline 105 & & signals _.. & Aug. 26,1890 \\
\hline 10 & & -6 & Aug. 26, 1890 \\
\hline 111 & Bu & & Aug. 26, 1890 \\
\hline & I & rmers, stationar & A ug. 2 \\
\hline & & itures - & Aug. 26, 1890 \\
\hline 132 & $-\ldots$ & Cut-outs, mechanical ........ & Aug. 26,1890 \\
\hline & & _. - do & A ug. 26 , I 890 \\
\hline & & erhead, trolleys & Aug. 26,1890 \\
\hline & - & is -- & Aug. 26, 1890 \\
\hline & & & Aug. 26,1890 \\
\hline 23 & & $\mathrm{Lig}$ & Aug. 26, 1890 \\
\hline & & vity feed, gear & A ug. 26, 1890 \\
\hline & -..--.. & -.... & Aug. 26,1890 \\
\hline & & $--1--1--1$ & Aug. 26,1890 \\
\hline & & & Aug. 26 \\
\hline 63 & $J$ & sed conduit & Aug. 26, 1890 \\
\hline & C. J & Metal working, miscellaneous & Aug. 26, 1890 \\
\hline & & $\mathrm{He}$ & Aug. 26,1890 \\
\hline & ---. & & Aug. 26, 1890 \\
\hline & & Tele & Aug. 26,1890 \\
\hline & 品 & ems, automatic ......- & Aug. 26, 1890 \\
\hline & & Mag & Aug. 26, 1890 \\
\hline & & $\operatorname{Re}$ & Aug. 26, 1890 \\
\hline 435,338 & $-\infty-\infty$ & Printing, matrix making & Aug. 26, 1890 \\
\hline & Wilst & Railway signals ....-..-- & Aug. 26, 1890 \\
\hline & & Electrodes & Aug. 26 \\
\hline 46 & - & Batteries, gal ranic & Aug. 26,1890 \\
\hline
\end{tabular}




\begin{tabular}{|c|c|c|c|}
\hline No. & & Class. & DATE. \\
\hline & Robb, J. & Liglits, incandescent, special featu & Aug. 26, 1890 \\
\hline $5,376^{2}$ & Brown, F.I & Electrodes & Aug. 26,1890 \\
\hline 11,108 & Higham, D & Regulators ..... & Aug. 26,1890 \\
\hline 435,421 & Hewett, E. M & Batteries, galvanic & Sept. 2,1890 \\
\hline+24 & & Cut-outs, mechanical & Sept. 2,1890 \\
\hline 438 & Lockw & Telephones, electric --- & Sept. 2,1890 \\
\hline$\$ 40$ & Lut $]$ & Annunciators & Sept. 2,1890 \\
\hline & $\mathrm{P}$ & Railways, systems, closed conduit - & Sept. 2, 1890 \\
\hline 771 & II & Railways, systems, multiple arc_-- & Sept. 2,1890 \\
\hline 482 & $\mathrm{C}$ & ignals & Sept. 2,1890 \\
\hline & Elliott, W. I & s, systems, closed conduit - & Sept. 2, 1890 \\
\hline 487 & Elliott, T. I & 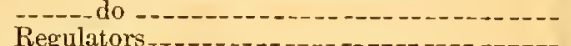 & Sept. 2, 1890 \\
\hline 90 & $\begin{array}{l}\text { Foot } \\
\text { Lieb }\end{array}$ & $\begin{array}{l}\text { Regulators } \\
\text { Magneto-electric }\end{array}$ & Sept. 2, 1890 \\
\hline 5,503 & $\mathrm{Li}$ & Magneto-electric ...-..- & $\begin{array}{l}\text { Sept. 2, } 1890 \\
\text { Sept. 2, } 1890\end{array}$ \\
\hline 5,505 & Lieb, C. A & Conductors, supports and ins & Sept. 2,1890 \\
\hline 516 & Perls, $\mathrm{T}_{---}$ & signals & Sept. 2,1890 \\
\hline 518 & $\mathrm{I}$ & ation- & Sept. 2, 1890 \\
\hline & C. I & Regula & Sept. 2, 1890 \\
\hline 526 & $\mathrm{CE}$ & -----do - - - & Sept. 2,1890 \\
\hline 527 & C. & - - do $-1-1$ & Sept. 2,1890 \\
\hline 536 & 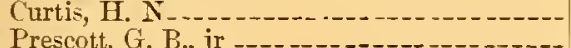 & ems, co & Sept. 2, 1890 \\
\hline $5+5$ & G. II $=\ldots$ & +1 & $\begin{array}{l}\text { Sept. 2, } 1890 \\
\text { Sept. 2, } 1890\end{array}$ \\
\hline 5.50 & $\mathrm{~F} \overline{7}^{\circ}$ & signals & $\begin{array}{l}\text { sept. } 2,1890 \\
\text { Sept. } 2,1890\end{array}$ \\
\hline &.- & ystems, overhead, trolley & Sept. 2, 1890 \\
\hline 578 & $\frac{E}{\mathrm{C}}$ & kets and keys & Sept. 2, 1890 \\
\hline 582 & $\mathrm{~L}$ & checks . . & Sept. 2,1890 \\
\hline 629 & 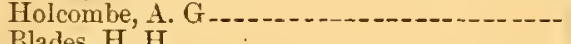 & ctors $=-\ldots \ldots-\ldots$ & Sept. 2, 1890 \\
\hline & $\mathrm{B}$ & $-1-1-1-1-2$ & Sept. 2, 1890 \\
\hline 40 & -- & ocomotives -..-- & Sept. 2, 1890 \\
\hline 11 & $---1--1$ & 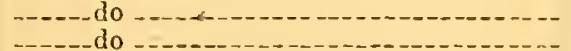 & Sept. 2, \\
\hline & --- & $-1-$ & $\begin{array}{l}\text { Sept. 2, } 1890 \\
\text { Sept. 2, } 1890\end{array}$ \\
\hline 544 & T & do.--- & $\begin{array}{l}\text { Sept. } 2,1890 \\
\text { Sept. } 2,1890\end{array}$ \\
\hline & $\lambda$ & nd carbons & Sept. 2,1890 \\
\hline 62 & 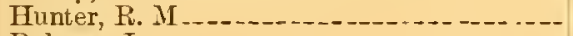 & conduit -- & Sept. 2,1890 \\
\hline 68 & $\mathrm{~F}$ & at, spec & Sept. 2,1890 \\
\hline 79 & & is and $s$ & Sept. 2, 1890 \\
\hline 87 & $t$ & y, applications -- & Sept. 2, 1890 \\
\hline & F & ic $-\ldots . . .-$ & Sept. 2, 1890 \\
\hline 89 & 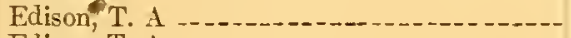 & plex --... & Sept. 2, 1890 \\
\hline & & etric & Sept. 2, 1890 \\
\hline & I & ters & Sept. 2, 1890 \\
\hline 0 & --- & -..- & Sept. 2, 1890 \\
\hline & & $\mathrm{N}$ & Sept. 2,1890 \\
\hline & & $\mathrm{P}$ & Sept. 2, 1890 \\
\hline & & & Sept. 2,1890 \\
\hline & $\mathrm{H} \ldots .$. & $\mathrm{t}$ clo & Sept. 2, 1890 \\
\hline 0 & - - - & ulators & Sept. 2,1890 \\
\hline & & and columns ....... & Sept. 2,1890 \\
\hline & $x^{2}$ & as, conduit _-_-_--_- & Sept. 2, 1890 \\
\hline & $-\infty$ & , calls ........ & Sept. 2, 1890 \\
\hline & & do & Sept. 2,1890 \\
\hline & --- & $G+\frac{1}{2}$ & Sept. 2,1890 \\
\hline & & atic & Sept. 2,1890 \\
\hline & $j$ & rollers _.---... & Sept. 2, 1890 \\
\hline & --- & & Sept. 2,1890 \\
\hline & & sockets and keys & Sept. 9, 1890 \\
\hline & -- & 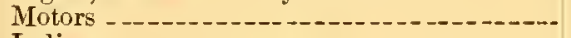 & Sept. 9, 1890 \\
\hline & $-\infty$ & & Sept. 9, 1890 \\
\hline & D & Lights, arc, gravity feed, gear-clutch & Sept. 9, 1890 \\
\hline & $-\ldots$ & Regulators -- - & Sept. 9,1890 \\
\hline & ------1 & ission 0 & Sept. 9,1890 \\
\hline & $\mathrm{K}$. and Hill, F. $\mathrm{M}$ & $\ldots$ & $\begin{array}{l}\text { Sept. 9, } 1890 \\
\text { Sept. 9, } 1890\end{array}$ \\
\hline & (2) & & Sept. 9,1890 \\
\hline & & - & Sept. 9,1890 \\
\hline & & A & Sept. 9,1890 \\
\hline & P $P$ - & condary _-.... & Sept. 9,1890 \\
\hline & nd Perkins, C. G & hanical & Sept. 9,1890 \\
\hline & & & Sept. 9,1890 \\
\hline & $-20-0-0-1$ & do _- & Sept. 9,1890 \\
\hline & & & Sept. 9, 1890 \\
\hline & & & $\begin{array}{l}\text { Sept. } 9,1890 \\
\text { Sept. } 9,1890\end{array}$ \\
\hline 436,122 & 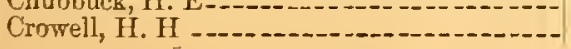 & Cut-outs, mechanical & Sept. 9, 1890 \\
\hline
\end{tabular}




\begin{tabular}{|c|c|c|}
\hline No. & NAME. & Cluss. \\
\hline 36,125 & ey, M. IV & Heaters - \\
\hline & Edison, T. A. & Transmission of power \\
\hline 48 & Newton, E. C. & Magneto-electric, couplings \\
\hline 154 & Schroen, F. C & 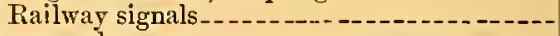 \\
\hline & Burt, E. M & 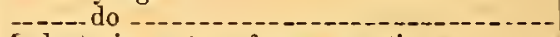 \\
\hline 200 & inghonse, & Inductoriums, transformers, stationary ...... \\
\hline 20.5 & Berlin, C. F - & Applications, general -- \\
\hline & Deeds, H. WV & e controllers \\
\hline 238 & Hoop, G. D - & Telegraphy, lightning arresters \\
\hline 275 & Van Depoele, & Transmission of potrer \\
\hline 276 & Tan Depoele, & do \\
\hline 277 & Tan Depoele, & 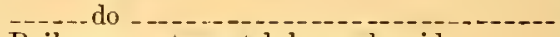 \\
\hline 81 & Williams, J. T & lenoid ........ \\
\hline & right, D. J. and Keyes, B. B. & - n- \\
\hline 319 & Silkman, E. J. & riting \\
\hline $3 \pm$ & Burnley, W.. & Telephones, electric \\
\hline 35 & ey, IV & 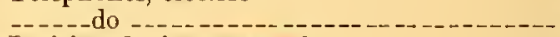 \\
\hline 54 & en, M. M & Igniting devices, manual. \\
\hline 71 & Winton, H. D & 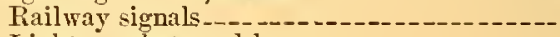 \\
\hline 87 & 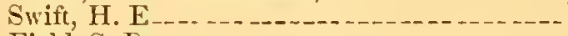 & 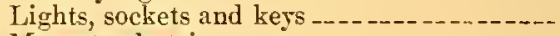 \\
\hline 08 & , S. $D_{--}$ & 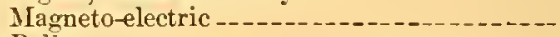 \\
\hline 10 & $\mathbf{J}$ & $\mathrm{Be}$ \\
\hline 12 & F. D'A & and keys \\
\hline 25 & , H. W. & 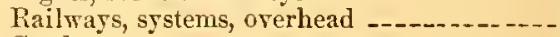 \\
\hline & W. E. & 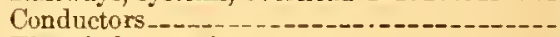 \\
\hline 39 & 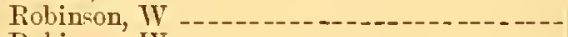 & ric locomotives \\
\hline 40 & 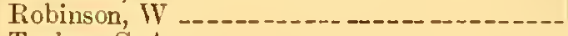 & - do - - - - \\
\hline 65 & $---n-\cdots$ & ts, arc, positive feed \\
\hline & nd, S. L & gnetic - - - - - - - \\
\hline 13 & ad, S. L & es, electric _- \\
\hline 14 & 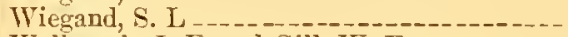 & es, relays and repeaters........... \\
\hline 16 & and Gill, II. E,$\ldots$ & es, galvanic \\
\hline 19 & 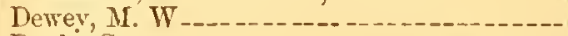 & 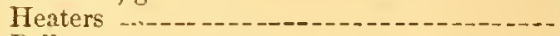 \\
\hline 60 & $\mathrm{G}_{-\ldots}-\ldots$ & Is --1--1- - - - n- \\
\hline 71 & D. A & tems, overhead, trolleys \\
\hline 602 & gsliead, & es, secondary \\
\hline $6 \pm 0$ & -------1 & pliy, fire \\
\hline $67 T$ & urr, E. B. & incandescent, special features \\
\hline 03 & 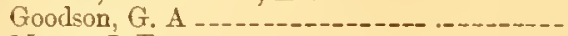 & g, matrix-making \\
\hline 17 & 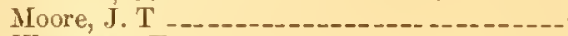 & pports _ _- \\
\hline 28 & - & otives \\
\hline 33 & 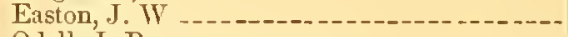 & 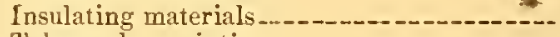 \\
\hline 740 & 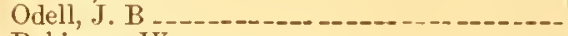 & phy, printing- \\
\hline 742 & nson, IV & Electric locomotives \\
\hline 743 & Robinson, II - & - $-1-\mathrm{d} d 0$ \\
\hline $7 \pm 7$ & 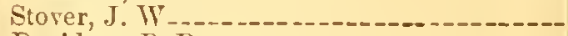 & Telegrapliy, fire \\
\hline 777 & 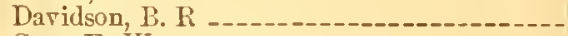 & signals._. \\
\hline-79 & E. W & - - - - - - - - - \\
\hline 804 & s, M. J. & ts_- \\
\hline $31 \pm$ & ere, $\mathrm{A}$ - & ity feed, clutch \\
\hline 43 & R. D. O & ions, tools and machines \\
\hline 57 & F. R . . & ectors_- \\
\hline 364 & gen, E- & 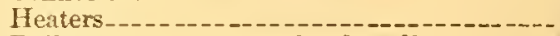 \\
\hline 874 & , J. J & systems, orerhead, trolleys \\
\hline 895 & J.A & deposition _ n \\
\hline 901 & S. E - & Regulators \\
\hline 910 & re, $\mathrm{II}_{\text {- }}$ & as \\
\hline & H. IV & s, systems, conduit _....... \\
\hline & $\mathrm{E}$ & gas engines, explosive \\
\hline 14 & & ss, srstems, orerhead \\
\hline 52 & 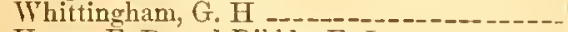 & aal \\
\hline 961 & a, F. R. and Dibble, F. J -- & ohy, fire, alarms and extinguishers - \\
\hline 964 & C.-.- & electric - \\
\hline 79 & C. $G=$ & Bells \\
\hline 10 & , E. M & es, systems \\
\hline & E. MI & 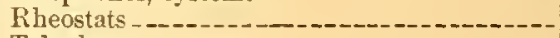 \\
\hline 12 & $M \ldots$ & Telephones, systems_-_. \\
\hline 11 & A, F. I & Insulating materials_._. \\
\hline 669 & brock, F. IV.-- & distance instruments...-_...-. \\
\hline 979 & ook, L. D $\ldots$ & 1 and surgical, body-rrear _..-_- \\
\hline & J. H - & 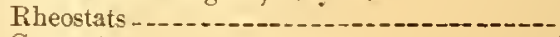 \\
\hline 116 & $y$, J. J & - \\
\hline & $\mathbf{E}=--$ & hetors, nnderground ... \\
\hline & R. M. & ic locomotives....... \\
\hline & , M & Motors _......... \\
\hline & burn, $F$ & ompasses -. - - \\
\hline & Dy & \\
\hline
\end{tabular}

Date.

Sept. 9,1890 Sept. 9,1890 Sept. 9, 1890 Sept. 9, 1890 Sept. 9, 1890 Sept. 9,1890 Sept. 9,1890 Sept. 9,1890 Sept. 9,1890 Sept. 9,1890 Sept. 9,1890 Sept. 9,1890 Sept. 9,1890 Sept. 9,1890 Sept. 9,1890 Sept. 16,1890 Sept. 16, 1890 Sept. 16, 1890 Sept. 16,1890 Sept. 16, 1890 Sept. 16,1890 Sept. 16,1890 Sept. 16, 1890 Sept. 16, 1890 Sept. 16.1890 Sept. 16, 1890 Sept. 16, 1890 Sept. 16,1890 Sept. 16, 1890 Sept. 16,1890 Sept. 16,1890 Sept. 16,1890 Sept. 16, 1890 Sept. 16, 1890 Sept. 16,1890 Sept. 16,1890 Sept. 16, 1890 Sept. 16, 1890 Sept. 16,1890 Sept. 16, 1890 Sept. 16, 1890 Sept. 16, 1890 Sept. 16, 1890 Sept. 16, 1890 Sept. 16, 1890 Sept. 16,1890 Sept. 23, 1890 Sept. 23, 1890 Sept. 23,1890 Sept. 23, 1890 Sept. 23, 1890 Sept. 23, 1890 Sept. 23,1890 Sept. 23, 1890 Sept. 23, 1890 Sept. 23, 1890 Sept. 23, 1890 Sept. 23, 1890 Sept. 23,1890 Sept. 23, 1890 Sept. 23, 1890 Sept. 23, 1890 Sept. 23, 1890 Sept. 23, 1890 Sept. 23, 1890 Sept. 23, 1890 Sept. 23,1890 Sept. 23, 1890 Sept. 23,1890 Sept. 23, 1890 Sept. 23,1890 Sept. 23, 1890 Sept. 23, 1890 Sept. 23, 1890 Sept. 23, 1890 Sept. 23, 1890 Sept. 30,1890 
$+37,203$

437,204

437,005

437,206

437,210

437,206

431,259

43,27 ?

437,293

437,307

$43-, 311$

$437,3: 4$

437,330

437,348

43-.3.

$437,3.58$

437,359

437,360

$43-362$

437,363

43ร, 369

437.387

437,393

437,397

$457,+1$ ?

$437,+202$

437.428

$437,4+5$

$43-469$

437.501

$+37,502$

437.510

$4:-512$

437,513

$43-516$

$+37,526$

437,533

437,534

$437,53.5$

437,563

437,570

437,571

437,602

437,613

$435,63 ?$

$437,6.54$

$\pm 37,661$

$437,66^{\circ}$

$+37,66.3$

437,667

437,668

437,67 .

437,6952

$43 \overline{7}, 68$.

$437, \pi 04$

437,720

$43 \bar{i}, \overline{0} 4$

$43-, 663$

437,767

497,71

$437,-89$

437,798

437,832

437,835

437,541

437,848

$437,5.51$

$437,8.59$

437,868

437,983

$43-, 901$

137,905

$43-, 913$

$+37,926$
Hunter, G. E. and Corthell, F. H.

Hunter, G. E. and Corthell, F. H

Hunter, G. E. and Corthell, F. H

Hunter, G. E. and Corthell, F. H

Leber, L. H

IIcEntee, J. A

prague, J. J

Wilking, F. and Yüller, H -

Dugan, H. F

Kunhardt, D

IIeCluer, C. E

Bailev, E. E. and Galbraith, J

Brooks, D., jr

Powell, S. L

Battershall, J. IV

Harding, C. $\mathbf{K}$

Hochhausen, II

Hochliausen, IV

Jenks, TV. J

Kalbach, H. UI

Wschira, A

Crosby, E. H

Hudic, $\mathbf{R}$

Deprez, II

Edison, T. A

Edison, T. A

Morton, T. M

Liebig, G. A. and Willms, C

Morder, WT. M

Mosher, J. A

Rae, F. B

Rae, F. B

Sawver, R. F

Spang, H. II

Ian Depoele, C. J

an Depoele, C. J

Van Depoele, C. J

Clark, E

Coffin, C. L

Coffin, C. L

Uurray, J. W

Splitdorf, C. F

Lander, G. and Simpson, J. H

Rae, F. P

Rae, F. P

Rae, F. B

Stanley, L. T

Pell, L. and Root, F. H

Damon, G. L

Gaynor, T. F

Lundell, $\mathrm{R}$

Reed, E. MI

Einstein, J. and Kornprobst, S

Riekenzaun, A

Beach, R. H

Butterfield, H.

Nichols, A. E

Ransom, G. F

Emmner, J., jr

Little, G. and G. J -

Blades, H. H

Lampert, H., jr

Brown, J. E

Kelley, D. and Parkhurst, M. C.-...........

Pitchie, A. II

Brady, T. H

Hazeltine, C. W

Levi, M

Prooks, D,

Harris, $\mathrm{T}$..

Hughes, F. C

Miner, W. M

Swett, E. A
Electric clocks

-

Electric locomotives

Advertising, signs .

Gearing

Batteries, secondary, applications

Vending apparatus

Telegraphy, aut

Conductors, anti-inductive

Cut-outs, thermal .

Conductors, lead-covered

Railway signals

Cut-ouss, mechanical

Railways, systems, closed conduit

Cut-outs, mechanical

Magneto-electric

Telegraphy, lightning aresters.........

Cut-outs, thermal

Alirms

Machines for winding

Batteries, galvanic

Telegraphy, lightning ariester's

Rheostats

Telegrapliy, cireuits and systems

Clutehes, intermittent-grip

Conductors, underground

Batteries, galvanic

Magneto-electric

Lights, arc, positive feed, gear-cluteh

Telegraphy, key

Telegraphy, dynamo

Cut-outs, mechanical

Electric lighting, systems

Lightning rods

Railways, conductor supports and insulators

Railways, systems, overhead, trolleys

Electric locomotives

Conductors

Heaters

$$
\text { do }
$$

Telephones, details

Railways, systems, overhead_.

Conductors -

Heaters

Electric locomotive

Mutors

Generation, magneto-electric

Cut-outs, mechanical

Telegraphy, tire, alarms and extinguishers

Valve-controllers

Hagnetic locks and bolts

Insulators

Regulators

Railways, systems, conduit ...............

Meters -..-_ -

Lights, arc, positive feed

Heaters

Lights, are, special features ............

Annunciators --

Motor

Iagneto-electric

Batteries, secondary

Lights, incandescent, special features .....

Telegraphy, dial

Galvanometers

Supports and hangers

Lights, arc, special features

Automatic devices

Conductors

Galvanometers

Telephones, supports

Telegraphy, mnltiplex

Generation, magneto-electric

Sept. 30, 1890

Sept.-30, 1 s.90

Sept. 30,1890

Sept. 30, 1890

Sept. 30, 1890

Sept. 30, 1890

Sept. 30, 1890

Sept. 30, 1590

Sept. 30, 1890

Sept. 3(), 1890

Sept. 30, 1890

Sept. 30,1890

Sept. 30, 1890

Sept. 30,1890

Sept. 30, 1890

Sept. 30, 1890

Sept. 30, 1890

Sept. 30, 1890

Sept. 30, 1890

Sept. 30, 1890

Sept. 30,1890

Sept. 30, 1890

Sept. 30,1890

Sept. 30, 1890

Sept. 30, 1890

Sept. 30,1890

Sept. 30, 1890

Sept. 30, 1890

Sept. 30,1890

Sept. 30,1890

Sept. 30,1890

Sept. 30,1890

Sept. 30, 1890

Sept. 30,1890

Sept. 30, 1890

Sept. 30, 1890

Sept. 30,1800

Sept. 30, 1890

Sept. 30,1890

Scpt. 30,1890

Sept. 30, 1890

Sept. 30, 1890

Sept. 30, $18: 0$

Sept. 30, 1890

Sept. 30,1890

Sept. 30,1890

Sept. 30, 1890

Sept. 30,1890

Sept. 30, 1890

Sept. 30,1890

Sept. 30,1890

Oet. 71890

Oct. $\quad$ 7. 1890

Oct 7,1890

Oet $\quad 1890$

Oct. 7,1890

Oct -1890

Oct. 7,1890

Oct. 7,1890

Oct 7,1890

Oet. $7,18 \% 0$ 
No. NAME.

Class.

DATE.

437,953 Wehrle, J. H

437,961 Buker, I. F

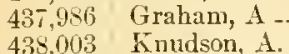

438,036 Verstraete, E

438,037 Vetter, J. C

438,041 Watkins, R. L

438,084 NicElroy, D.S

438,116 Slıpleigh, W

438,117 Shapleigh, W

438,118 Smith, W. F

438,127 Ackermann, W. J

438,134

438,145

438,167

438,174

438,192

439,204

438,211

438,219

438,226

$438,-23$

438,234

435,236

438,260

438,262

438,293

438,298

438,299

438,301

438,302

439,303

438,304

$438,30.5$

438,306

438,307

438,309

435,309

485.311

435.312

$438,31:$

435,314

438,320

438,34

$438,3$.

438,35 .

438,361

438,390

438,394

Apps, A

currie, S. C.

Parteler, T

Shawlian, J. F

Thomson, F.

Wirt, H. C

Andersen, J. M

Currie, S. C. C.

Geary, J

Wheless, M

Sprague, F. J

Edison, T. A

Edison, T. A

Edison, T. A

Edison, T. A

Edison, T. A

Edison, T. A

Edison, T. A

Elison, T. A

Edison, T. A

Edison, T. A

Enholm, O. A

Enholm, O. 1

Enholm, O. A

Ripperich, A R.

Chaize, $\mathbf{N}$. and $\mathbf{J}$

M1cMahan, J. MI

Pfingst, I

Plunb,

Stanley, A. F

White, W. I

Breunen, G. J

Dewey, MI. IT

Dewer, M. IV

Dewey, M. II

Dewey, MI. II

Ginoclio, N. B

Short, S. H

Burton, G. D

curric, s. C. C

Faunce $\mathrm{F}$

Mrorphy, R.

Woods, G. T

Ashton, F. MI

Bradley, C.

Bradley, C.

Giles, J. E

MacDonald, A.J

MacDonald, A. J

Striemer, $\mathrm{A}$

Tliomson, E
Hollo $\mathrm{W}$ H

Hollar, W. H. and Holmes, F.

Edison, T. A. and Gilliland, E. T

Edison, 'T. A

Van Depoele, C.J

Crockett, C. H. and Dedrick, L. C _._.....

Eckert, W. H. and Gregory, W. H _.......
Railways, systems, conduit

Railways, systems, overhead, trollers

Bridges, girders and columns

Rheostats _...- Oet. 7, 1890

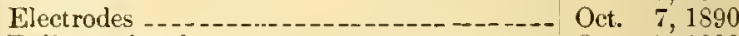

Railway signals .......................... Oct. F, 1890

Batteries, secondary, applications _.........- Oct. 7, 1890

Metallurgy, lead_... T, 1890

Cut-outs, mechanical _..._._._._._._._... Oct. T, 189ij

Alarms, burglar _....................... Oct. 7, 1890

Lights, filaments and carbons -...-...-.- Oct. 14,1890

Lighting, systems, secondary battery _..._. Oct. 14, 1890

Cut-outs, mechanical _._._._........... Oct. 14, 1890

Vending apparatus ...................... Oct. 14, 1890

Railways, systems, battery _._............ Oct. 14, 1890

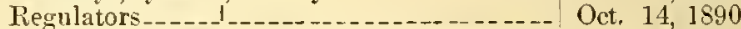

Railways, conductor supports and insulators. Oct. 14, 1890

Railways, svstems, overhead, trolleys _.....- Oct. 14, 1890

Galvanometers -

Telegraphy, relays and sounders......... Oct. 14, 1890

llarms, burglar - Oct. 14, 1890

Magnetic locks and bolts .................. Oct. 14, 1890

Railways, switebes, tramway _........... Oct. 14, 1890

Railwars, systems, circuits, multiple arc -... Oct. 14,1890 do

Iights, incandescent

Oct. 14,1890

Oct. 14,1890

Oct. 14,1890

Oct. 14, 1890

Oct. 14,1890

Oct. 14, 1890

Oct. 14,1890

Oct. 14,1590

Oct. 14,1890

Oct. 14, 1890

Oct. 14,1890

Oct. 14,1890

Oct. 14,1890

Oet. 14,1890

Oct. 14,1890

Oct. 14,1890

Oct. 14,1890

Oct. 14,1890

Oct. 14,1890

Oct. 14,1890

Oet. 14,1890

Oct. 14,1890

Oct. 14,1890

Oct. 14,1890

Oct. 14,1890

Oct. 14, 1890

Oet. 14,1890

Oct. $1 \pm, 1890$

Oet. 14,1890

Oct. 14, 1890

Oct. 14,1890

Oct. 14,1890

Oct. 14,1890

Oct. 14,1890

Oct. 14,1890

Oct. 14,1890

Oct. 14,1890

Oct. 11,1890

Oct. $1 \pm, 1890$

Oct. 14,1890

Oet. 14,1890

Oct. 14,1890

Oct. 21,1890

Oct. 21, 1890

Oct. 21, 1890

Oct. 21,1890

Oct. 21, 1890

Oct. 21,1890

Oct. 21, 1890

Time alarms -..... Oct. 21, 1890 


\begin{tabular}{|c|c|c|c|}
\hline No. & NAME. & Class. & DATE. \\
\hline & & Heaters & Oct. 21,1890 \\
\hline & Thomson, E & --- do $_{-}$ & Oct. 21,1890 \\
\hline & Winkler, C. F - & Magueto-electric & Oct. 21,1890 \\
\hline & Menuez, A. E & Insulating materials & Oct. 21,1890 \\
\hline 438 & Whittingham, G. & Cut-outs, thermal & Oct. 21,1890 \\
\hline & Burton, G. D -- & Heaters_-_-_-_--:---..- & Oct. 21,1590 \\
\hline & Popp, I $Y^{\top} \ldots$ & Clocks, pneumatic _... & Oct. 21,1890 \\
\hline & 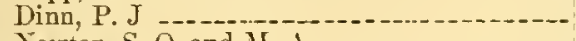 & Lamp posts . . & Oct. 21, 1890 \\
\hline & wton, S. O. and & Drop lights and hangers.... & Oct. 21,1890 \\
\hline & Schmid, F. MI & Electro-magnets _........-. & Oct. $21,1 s 90$ \\
\hline & ut, J. C. $\mathrm{H}_{-\ldots}$ & Telephones, electric & Oct. 21,1890 \\
\hline & White, A. C - -- & Telegraphy, lightning arresters & Oct. 21,1890 \\
\hline 438,814 & in, $M$ & $\mathrm{~g}$, systems. - & Oct. 21,1890 \\
\hline & Melonough, J. & Telep & Oct. 21,1890 \\
\hline & Rernier, E. $\mathbf{Y}$ & Batteries, secondary & Oct. 21,1890 \\
\hline 25 & W. L. & 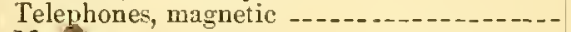 & Oct. 21,1890 \\
\hline & & Motors & Oct. 21,1890 \\
\hline 438,847 & R. M -- & Railways, systems, overhead... & Oct. 21,1890 \\
\hline 433 & $\mathrm{E}_{--\ldots}$ & oid... & Oct. 21,1890 \\
\hline & Fr & & Oct. 21,1890 \\
\hline & S.H & ncandescent, special features.-. & Oct. 21,1890 \\
\hline & H. IV $=$ & systems, 0 & Oct. 21,1890 \\
\hline & $\mathrm{Is}, \mathrm{F} . \mathrm{H}$ & $s$ hon & Oct. 21,1890 \\
\hline 34 & --.-- & $\mathrm{C}$ & Oct. 21,1890 \\
\hline & F. G & $\mathrm{Te}$ & Oct. 21.1890 \\
\hline 439 & -1 & lutch. & Oct. 21,1890 \\
\hline &..-- & --- & Oct. 21,1890 \\
\hline & avies, & $\mathrm{T}$ & Oct. 21,1890 \\
\hline $43 ?$ & D. B - & d hangers--- & Oct. 21,1890 \\
\hline & - & ting & Oct. 21,1890 \\
\hline 439 & F. and Terline, $\mathrm{E}$ & and keys ........ & Oct. 21,1890 \\
\hline $439,05 \overline{5}$ & rschau, $A$ & $\mathrm{ks}$ & Oct. 21,1890 \\
\hline & & $\mathrm{R}$ & Oct. $2 !, 1890$ \\
\hline & -------- & e arc & Oct. 21,1890 \\
\hline & A. $F_{-}$ & $y_{\text {- }}$ & Oct. 28,1890 \\
\hline & $C \mathrm{~S}$ & g. & Oct. 28,1890 \\
\hline & W. L. & $\mathrm{Bat}$ & Oct. $\mathbf{2 8}, 1890$ \\
\hline & ----1 & $\mathrm{Li}$ & Oct. 28,1890 \\
\hline & ---1 & $\mathrm{Ap}$ & Oct. 28,1890 \\
\hline & .... & $\mathrm{s}$, overhead, $\mathrm{t}$ & Oct. 28,1890 \\
\hline & ralloupe, $\mathrm{F}$ & do - & Oct. $2 S, 1890$ \\
\hline 43 & 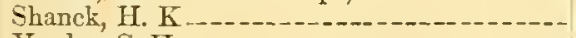 & A & Oct. 28,1890 \\
\hline 439 & $\mathrm{H}$ & es.-- & Oct. $2 \mathrm{~S}, 1890$ \\
\hline 439 & W- & plosive & Oct. 28,1890 \\
\hline & G.- & tery ---------- & Oct. 28,1890 \\
\hline & A - & $\mathrm{D}$ & Oct. 28,1890 \\
\hline 40 & D. $P$ & Batteries, secondary ... & Oct. 28,1890 \\
\hline $43 !$ & $d, L_{-}$ & s, conduit & Oet. $2 S, 1890$ \\
\hline 439,295 & $\mathrm{~F}$ & - - & Oct. 28,1890 \\
\hline & & $\mathrm{B}$ & Oct. 25,1890 \\
\hline & - On-. & 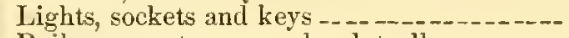 & Oct. 28,1890 \\
\hline & ------ & s, overhead, trolleys . - & Oct. 28,1890 \\
\hline & . $J$ & lary _-_. & Oct. $2 \mathrm{~S}, 1890$ \\
\hline & F. IF & & Oct. 28,1890 \\
\hline & $s$, J.F & Medical and surgical, body-wear...... & Oct. 28,1890 \\
\hline & ........ & Lights, sockets and keys & Oct. 28,1890 \\
\hline & 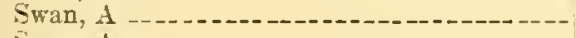 & -- & Oct. 28,1890 \\
\hline & 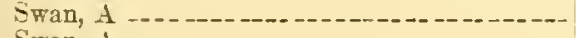 & do.-- & Oct. 28,1890 \\
\hline & $\ldots$ & & Oet. 2 \\
\hline & & & Oct. 28,1890 \\
\hline & $\mathrm{B}$ & 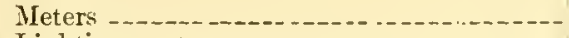 & Oct. 28,1890 \\
\hline & $\mathrm{F}$ & & Oct. 28 , \\
\hline & E & y'stems, secondary battery - & Oct. $2 S, 1890$ \\
\hline & & ind -............ & Oct. $2 S, 1890$ \\
\hline & & & Oct. 28,1890 \\
\hline & & and carbons ------- & Oct. $2 S, 1890$ \\
\hline & & s, battery .......... & Oct. 28,1890 \\
\hline & & C & Oct. $2 S, 1890$ \\
\hline & & Ra & Oct. \\
\hline & and Schmidt, L. & secondary_ & Oct. 28 , \\
\hline & Whon & Regulators-_-__-__- & Oct. 28,1890 \\
\hline & & Rail & Oct. 28,1890 \\
\hline & kill, C. W & , circuit closers, thermal _. & Oct. 28,1890 \\
\hline & and Lnbois, $\mathrm{H}$ & s, throttle -..- & Oct. \\
\hline & & & Oct. 2 \\
\hline & Coff & Vending apparatus_- & Oct. 28,1890 \\
\hline & & & Oct. 28,1890 \\
\hline
\end{tabular}




\begin{tabular}{|c|c|}
\hline No. & NaME. \\
\hline 439,516 & Hussey, C. A - \\
\hline 439,577 & -------- - - - \\
\hline $439, \overline{5} 84$ & Wagemann, E - \\
\hline 439,594 & Hollingshead, WT. B _...- \\
\hline 439,597 & Hunter, R. MI \\
\hline $439,60^{\circ}$ & MeGregor, J. A. K _ \\
\hline 439,614 & Vaughn, B. E \\
\hline 439,662 & Hunter, R. M \\
\hline 439,724 & Drew, C. W. and Francis, E. $\mathbf{R}$ \\
\hline 439,726 & Elliott, G. R \\
\hline 439,737 & ------- - - - - - \\
\hline 439,746 & Emmet, W. Le R ....----------------- \\
\hline 439,775 & Gartner, A _- \\
\hline 439,792 & 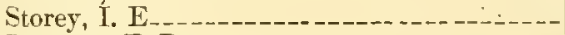 \\
\hline 439,796 & 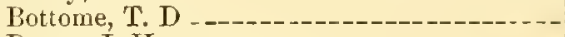 \\
\hline 439,838 & -- - - - - - - - - - - - - - -- \\
\hline
\end{tabular}

439,840

439,850

439,859

439,867

439,90

439,900

43901

439,429

439,939

439,941

439,959

439,974

440,013

$4 \pm 0,01$

440,016

440,023

440,024

$4 \pm 0,04$

410,070

440,071

440,091

$\$ 40,090$

440,100

$440,1] 5$

440,118

440,16

$4+0,16$

$\$ 10,173$

440,175

440,189

110,199

440,208

$\pm 1(1,2] 0$

440,21 .

410,216

440,22

440,24

440,242

$+10,268$

$\pm 10,20$ :

440,270

$\pm 40,271$

410,272

$\pm 40,273$

$410,2 \%$

440,275

$1 \pm 0,2 ; 6$

440,277

410,289

$+40,290$

440,291

440,303

440,326

$\pm \pm 0,361$

440,362

$\pm 40,375$

410,384

$\pm 40,391$

410,395

440,420

$440,4 ? 4$
Granville, IV. P

Woolf, A. E

Bossert, IV. F

Dewey, M. II

Shubert, H. C

Sprague, W. B. and Kellogg, C. W

Whittlesey, J. T

Cliild, C. T

Gilbert, E. R

Goldstone, L

Kohrn, W. A.J

Chatfield, $\mathrm{O}$

Picrce, F

Clearcl, F. J. and Fassold, G

Entz, J. B. and Plillips, TY. A

Entz, J. B. and Phillips, W. A

Tíessler, IV:

Ton Syo, E.

Talmage, C. H

Tinning $\mathrm{F}$. T and Tr.

Bergmann, $\mathrm{S}$

Ioyt, L. S

Pierce, F

Root, H. C

Keeley, D. H

Keeley, D. H

Macdonald, A. J

Marx, F

Peckham,

Fosebringl, A. MI

Tanderhoef,

Wheless, in

Woolf, A. I

De Ferranti, S. Z.

Schlegel, H. I

Wagg, S. R

Payen, C

Payen, C

Payen, $\mathrm{C}$

Payen, C

Payen, C

ayen,

Payen, C

Payen, C

Paven, $\mathrm{C}$

Payen, $\mathrm{C}$

Weston, E

IVeston,

Busl, A. R

Richards, W. S

MeNanman, J.A

Nourse, J. K. P

Smith, F. S

Wirt, C

Blaisdell, F. E

Cobb, H. B -

Cohlman, W.

Freeman, W. $\bar{K}$
Cluass.

DATE.

Batteries, galvanic

Applications, tools and machines

Electric locomotives

Batteries, secondary

Railways, systems, conduit _..._.......... Oct. 28, 1890

Cut-outs, mechanical _.............. Oct. 28, 1890

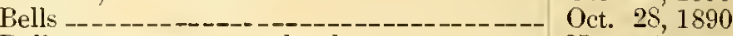

Railways, systems, overhead____.________-_ Nov. 4, 1890

Heaters _..................................... Nor. 4, 1890

Store service, gravity _._.___.___... Nov. 4, 1890

Batteries, galvanic --

Railways, systems, overhead _._._._._._. Nov. 4, 1890

Regulators _........... Nor. 4, 1890

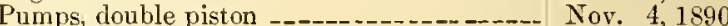

Insulating materials_-_-_--_-_-_..---_--_--- Nor. 4, 1890

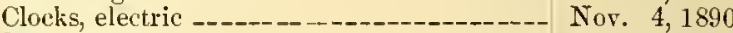

Ship's logs _._... Nov. 4, 1890

Battcries, secondary-_-_-_-_... Nov. 4, 1890

Cut-outs, thermal _._._. Nov. 4, 1890

Railways, systems, circuits, series -........- Nov. 4,1890

Lights, arc, positive feed, clutch _......... Nov. 4, 1890

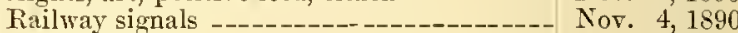

Railways, systems, overhead, trolleys _....... Nov. 4, 1890

Magneto-electric, couplings _............. Nov. 4, 1890

Rheostats _-_._- Nov, 4, 1890

Indicators _............... Nov. 4, 1890

Telegraphy, keys ............................... 4, 1890

Magneto-electric -

Telcgrapliy, fire, alarms and extinguishers_- Nov. 4, 1890

Alarms, burglar _._._._.............. Nov. 4, 1890

Regulators _._._. Nov. 4, 1890

Batteries, secondary-_--_--_-_-------------- Nor. 4, 1890

-_--do _-_- Nor. 4, 1890

Insulators...... Nov. 4, 1890

Separators --_-_-_-_-- Nov. 4, 1890

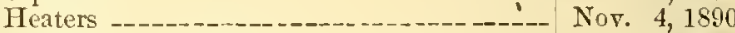

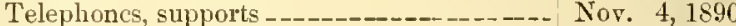

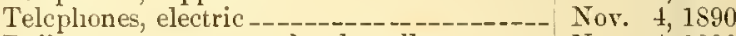

Railways, systems, overhead, trolleys--_--- Nor. 4, 1890

Alarms, burglar _.___._._._. Nov. 4, 1890

Tclephones, systems_-_-_._. Nov. 4, 1890

Telegrapliy, circuits and systems__-_._-__-_ Nov. 11, 1890

Telcoraphy, multiplex _............. Nov. 11; 1890

Alloys _._. Nov. 11, 1890

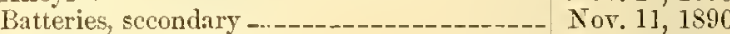

Electric locomotices._._._._._._._._. Nov. 11, 1890

Telegraphy, circuits and systems -.....-- Nor. 11, 1890

Indicators _........... Nov. 11, 1890

Batteries, secondary-_-_-_-_-_-_-_-_-_-_. Nov. 11, 1890

Railways, systems, circuits, multiple arc .... Nov. 11, 1890

Batteries, secondary _.___.__ Nov. 11, 1890

Srstems of distribution_-_-______._._. Nov. 11, 1890

Clocks, electric _._._._._-_._-_._._._._. Nor. 11, 1890

Paper making, digesters -..-_-_-_..--_-_--- Nov. 11, 1890

Metallurgy, lead..._.................. Nov. 11, 1890

Nor. 11, 1890

Nov. 11,1890

Nov. 11, 1890

Nov. 11,1890

Nor. 11, 1890

Nov. 11,1890

Nor. 11, 1890

Nov, 11,1890

Nov. 11, 1890

Nov. 11, 1890

Nov. 11, 1890

Nor. 11,1890

Nor. 11,1890

Nov. 11,1890

Nov. 11, 1890

Nov. 11, 1890

Nov. 11, 1890

Nov. 11, 1890

Nov. 11, 1890

Nor. 11, 1890

Nov. 11, 1890

Nov. 11, 1890 
40.425 Freeman, W.

40,431
$410, H 1$ Swès, $\mathrm{E}$

110.411

40,012

440,513

$\pm 10.5105$

$140,50 \mathrm{~s}$

$411,53 \pm$

110.573

440,563

40,975

140.551

$40, \overline{9} 90$

$440,59.5$

140,596

$40,59 \%$

440,604

110,614

$440,5 \div 2-7$

440,640

$140,6 \pm 1$

$40,65 \frac{1}{2}$

$40,66 \%$

110.663

$40.66 \pm$

140,665

4140,085

$140,686^{\circ}$

40,689

H4, (i91

410,699

$\$ 40,760$

440,717

440,718

410,720

440,753

110,75

$40,-66^{2}$

$410,-67$

$40,-63$

$410,-16$

40,780

$440,-81$

40,789

40,813

40,814

410,817

110,821

440,822

$140,51$.

40,881

40,895

$\$ 40,906$

40,308

4t), 925

140,926

40,927

40,984

40,976

$+40,9 ;$

411,030

41,031

111,032

411,033

41,031

441,043

111,041

411,059

141,060

441,066

411,122

441,123

411,127

441,128

441,130

441,157

111,158

Sargent, F

Freeman, IT. K

Paren, C-

Brooks, H. H

Hardy, F. D

Hunter, R. II

Hunter, R. II

Hunter, R. M

Lemp, $\mathrm{H}$

Lemp, H

Rice, E. W

Thomson, E

Baker, I. F

Belding, IV. S

Brown, H. P

Dressler, C. E

Dressler, C. E

Yower, S. E

Mower, S. E

Pflnger, $C$.

Bathurst,

Delany, P. B

Delany, P. B

Delany, P. B

Pallman, H. B

Verstraete, E

Terstraete, E

Cushing, P. D

Von Kohler, H

Lieb, C. A

Rae, F. B

Bremer, IV.

Herrick, C. H

Loomis, T. F

Trott, S -

Riggs, J. W

Riggs, J. W

Piggs, J. W

sheldon, W. D

Sheldon, W. D-

Sheldon, W. D

Sheldon, W. D

sheldon, W. I

Terstraete, $\mathbf{E}$

Collier, J. W

Conkling, $\mathrm{G}$

Hayes, H. V

Passett, X. C

Bomholdt, A

Bomholdt, A

Brewer, H. J

Hale, C. A
Ramsey, J., jr. and Weir, F. C

Ramser, J., jr. and Weir, F. C

Renault, B, and Desvernay, MI -

Bettendorf, W. P. and J. W

Hale, G. C. and Barret, $A$

Barberie, E. T. and Des Brisay, J

De Ferranti, S. Z

Thnmson, E. and Rice, E. W

Thomson, E. and Rice, E. W

Alton, G. H. and Rice, E. W

MeJanman, J. A

Andersen, F. V. and Girdlestone, J. $\mathrm{O}$

Tompkins, D. A -

Tan Depoele, C.

Waddell, W. H
Magneto-electric

Meters

Clocks, electric

Railway signals

$$
\text { -do }
$$

Batteries, secondary

Teleyraphy, railway-car

Heaters

Magneto-electric

Telegraphy, fire

Lead

Railways, systems, overhead, trolleys_._. ...

Regulators _. - _. -

Ralilways, systems, overhead

Railurays, systems, concluit

Lights, arc, positive feed

Cut-outs, mechanical

Meter:s

Heaters

Telegraphy, lightning arresters

Lights, arc, gravity feed, clutcl

Heaters

Railways, systems, overhead, trolleys.....

Cut-outs, thermal

Electric locomotives

Magneto-electric

Motors

Inductoriums, transformers, rotary

Electric locomotives

$$
\text { do }
$$

Cut-onts, mechanical

Lights, incandescent, special features

Lights, filaments and carbons _._..........

Telegraphy, multiplex

$$
\text { do }
$$

Motors

do

Railways, systems, conduit

Railways, systems, overhead, trolleys

Telegraphy, circuit closers

Railways, systems, overhead, trolleys...-.-

Magrieto-electric

Motors, reciprocating

Railways, systems, closed conduit

Cut-outs, mechanical

Railway signals

Telepliones, calls

Railways, systems, conduit

Magneto-electric

Railway signals

$$
\text { -do }
$$

Lights, incandescent, special features

Railways, systems

Transmission of power

Railway signals

Railway gates, electric

Railway signals

Conductors, underground

Railway signals,

Lights, sockets and keys

Separators

Cut-outs, thermal

Railways, systems, overhead, trolleys...-.

-- ---do do

Lights, incandescent

Batteries, galvanic -

Annunciators

Photography, combined apparatus

Xov. 11,1890

Nov. 11, 1890

Nov. 11, 1890

Nur. 11, 1890

Nov. 11, 1890

Nov. 11, 1890

Yov. 11, 1890

Nov. 11, 1890

Nov. 11, 1890

Yov. 11, 1890

Nov. 11, 1890

Nor. 11, 1890

Nov. 11, 1890

Nov. 11, 1890

Nov. 11, 1390

Nuv. 11, 1890

Nor. 11, 1890

Tov. 18, 1890

Nov. 18, 1890

Nov. 18, 1890

Nov. 1s, 1890

Nov. 18, 1890

Nov. 18, 1890

Nov. 18, 1890

Nov. 18, 1890

Nov. 18, 1590

Nov. 18, 1890

Nov. 18, 1890

Nov. 1s, 1890

Nov. 18, 1890

Nov. 18, 1590

Nov. 18, 1890

Nor. 18, 1890

Nov. 18, 1890

Nov. 1s, 1890

Nov. 1S, 1890

Nov. 18, 1890

Nov. 18, 1890

Nor. 18, 1890

Nov. 1s, 1890

Nov. 18, 1890

Nor. 1s, 1890

Nov. 18, 1890

Nor. 18, 1890

Nov. 1s, 1890

Nov. 18, 1890

Nor. 1s, 1890

Nov. 18, 1890

Nov. 18, 1890

Nov. 18, 1890

Nor. 18, 1890

Tov. 18, 1890

Nor. 1s, 1890

Nov. 18, 1890

Nov. 18, 1890

Nov. 18,1890

Nor. 18,1890

Nov. 18, 1890

Nov. 18,1890

Кот. 1s, 1890

Nov. 18,1890

Nov. 18, 1890

Nov. 18, 1890

Nor. 1\&, 1890

Nov. 18, 1890

Tor. 18, 1890

Nov. 18, 1890 
No.

NaME.

Class.

Date.

441,165 Jolnns, H. IV

441,210 Wheless, $\mathrm{M}$

441,211 Wheless, $M$

441,212 Wheless, $\mathrm{M}$

441,213 Wheless, M

441,214 Wheless, M

441,215 Wheless, M

$4+1,216$ Wheless, $\mathrm{M}$

441,217 Wheless, MI

$441,21 \mathrm{~S}$ Wheless, M

\begin{tabular}{l|l}
$4+1,219$ & Wheless, M \\
441,220 & Whealley, $\mathrm{S}$.
\end{tabular}

441,220 Wheatley, S. E

441,246 Lieb, C. A

441,245 Lymch, T. J

441,250 Nanaydicr, J. E

441,256
441,258 Reese, ${ }^{\prime} \boldsymbol{T}_{.,} \mathrm{j} \mathbf{r}$

441,259 Sylvester, P. L.

441,276 Bayles, J. C

441,277 Bayles, J. C

441,203 Doyle, $\mathrm{J}$

441,304 Holtzer, C. W

441,305 Hunter, R. MI

441,312 Iarks, W. B., Lauer, J. G. and Kalston, R. R-

$4+1,330$

441,391

$4+1,390^{\circ}$

$4+1,401$

$4+1,403$

$4+1,413$

$4+1,430$

441,458

$4+1,487$

$4+1,4,8$

$41,5 \pm 2$

$441,5+3$

41,565

441,571

441,572

441,578

441,579

$4+1,5$

441,591

441,622

$411,6+4$

411,665

411,695

441,703

$\pm 41, \pi \pm \overline{7}$

441,753

$\pm 1,764$

441,771

441,773

$4+1,793$

441,794

411,800

$414,80 \%$

$4 \pm 1,817$

$\pm 1, \$ 1 \mathrm{~S}$

41,828

41,897

411.838

$441, \therefore+0$

$41, \$ 43$

$411,8+5$

$4+1,8+9$

141,861

441,57

$4 \pm 1,892$

41,893

$\pm \pm 1,894$

41,908

$\pm 1,918$

411,919

$\pm \pm 1,935$

$4+1.055$

schleschinger, $\mathrm{W}$. M

Brown, C. E. I

Capps, F. L

Dewey, M. IV

Enholu, O. A

Hatch, G. E-

Mallonee, J. D

White, Le R. S

Hensler, W. S. and Grindley, W. H

Enholin, O. A

Enholm, O. A

Hunter, R. M

Libbey, II. IV

Libbey, H. II

O'Neil, J

O'Neil, J

Schultz, G

Sny.der, J. M

Conderman.

Hyer, IV. F

Patterson, IV

Butz, A. MI

Emmett, II. Le R

Jones, H

Nansfield,

Owen, R. O

Freeman, Wr. K

Kitfield, E. II

Pepper, D., jr

Bradbury, J. Y. and Stone, F. J

Dewey, il. II

Greentield, E. T

Greenfield, E. T-

Greenfield, E. T

Hoy, A. H

Jones, F. IT

Locke, F. MI. and Lapp, J

Tan Sant, W. S. and Eames, E. C

Lewis,

Possons, W.J

Possons, WT. $J$

Possons, II. J

Tiedemann, M. IT

Ames, T. K

Banta, II. I

Cartwright, D
Groswith, H
Boiler coverings -

Railways, systems, conduit

Railways, systems, circuits, multiple arc

Railways, systems, conduit

Electric locomotives

Railways, systems, closed conduit

Car brakes

Railways, systems, closed conduit

Railways, systems, circuits

Railways, systems, circuits, multiple arc -.. do

$$
\text { do }
$$

Railways, systems, conduit

Iagneto-electric

Railways, systems, telpher, solenoid

Magneto-electric

Railroads, systems, conduit..

Slidable pusher, coin engaged

Forging and welding -

Metal workiug, tubes.

Rhenstats .

Anuunciators

Railways, systems, battery

Conductors, supports and insulators

Electric locomotives

Magneto-electric

Telepliones, magnetic

Heaters

Batteries, galvanic

Batteries, secondary

Printing, hand stamps

lgniting devices, automatic

Iagneto-electric

Pyrometers

Systems of distribution

Lights, arc, gravity feed, clutch

Electric locomotives

Railways, srstems, conduit

-----do do

Compasses

Cut-outs, mechanical

Dampers, automatic

Telegraplyy, circuit closers, thermal .......

Storepipe thimbles

Regulators

Magneto-electric

Railway signials

Dampers, automatic.

Railurys systems, orerhead, trolleys -

Railnaýs, systems, conduit

Electric locomotives

Railrray siguals

Magncto-electric

Regulators

Conductors, supports and insulators

Regulators

Valve-controllers

Batteries, secondary

Railway's, systems

Conductors

Rlieostats -

Conductors, underground -...............

Batteries, gal ranic

Telegraphy, circuits and systems.....-.

lnsulators

Fluid deliverv-.............

Lights, incandescent, special features

Electro-deposition

-

Time alarms

Rheostats -

Connectors

Cut-onts, thermal

Motors
Nov. 25, 1890 Nov. 25, 1890

Nor. 25,1590

Nov. 25,1590

Nor. 25, 1890

Nov. 25, 1890

Nov. 25, 1890

Nov. 25, 1890

Nov. 25,1890

Nov. 25, 1890

Nov. 25, 1890

Nov. 25,1890

Nor. 25, 1890

Nov. 25, 1890

Nov. 25,1890

Nov. 25,1890

Nor. 25, 1890

Nov. 25, 1890

Nov. 25,1890

Nov. 25, 1890

Nor. 25, 1890

Nov. 25,1890

Nov. 25, 1890

Nov. 25, 1890

Nov. 25, 1890

Nov. 25, 1890

Tov. 25,1890

Nor. 25, 1890

Nor. 25, 1890

Nov. 25, 1890

Tor. 25, 1890

Tov 25, 1890

Tor. 25, 1990

Nor. 25, 1890

Nov. 25, 1890

Nor. 25, 1890

Nov. 25, 1890

Yov. 25, 1890

Nor. 25, 1890

Nov. 25, 1890

Nov. 25, 1890

Nor. 25, 1890

Nov. 25, 1890

Nov. 25,1890

Nov. 25,1890

Dec. 2, 1890

Dec. 2,1890

Dec. 2, 1590

Dec. 2,1590

Dec. 2, 1590

Dec. 2, 1590

Dec. 2, 1890

Dec. 2, 1590

Dec. 2, 1890

Dec. 2, 1890

Dec. 2, 1590

Dec. 2, 1890

Dec. 2, 1590

Dec. $\overrightarrow{2}, 1590$

Dec. 2, 1890

Dec. 2, 1890

Dec. 2, 1890

Dec. 2, 1890

Dec. 2, 1890

Dec. 2, 1890

Dec. 2, 1590

Dec. 2,1890

Dec. 2, 1590

Dec. 2, 1890

Dec. 2, 1890

Dec. 2, 1890

Dec. 2,1590

Dec. 2, 1890

Dec. 2, 1890

Dec. 2, 1890

Dec. 2, 1590

Dec. 2, 1890 
$41,9.5 \pm$ Groswith, $\mathrm{H}$

41,958 Kennedy, C. W. and Groswith, H

411,959- Kennedy, C. W

41,965

$41,97: 2$

411.973

$411,97-1$

$411,98:-2$

41,999

$412,00: 2$

412,018

412,029

44.030

$4: 2,053$

$4+20-1$

442,104

412,114

$4+2,115$

44,126

$442,1: 27$

442,139

412,140

$112,1+3$

442,141

442,145

412,146

412.155

112,162

42,173

412,187

412,203

$44: 2,204$

442,200

412,232

412,267

112,270

$412,25 \%$

$44: 332$

112,333

412,334

412,335

442,336

412,338

412,339

112,342

$442,34=$

412,365

442,370

442,383

412,395

112,390

412,391

$4 \frac{1}{2} 2,396$

442,407

142,415

442,423

442,146

$412, \pm 51$

442,452

442,459

$442,4 \div 2$

442,473

442,49

142,501

42,516

442,575

442,375

442,580

442,589

412,594

442,610

442,617

442,623

442,649

442,604

442,661

112,666

Pottome, T. D
De Peralta, C. G

Rapieft; J

Ravieff, J

Rapieff, J

Simpson, I. H.

Woud, J. J

dams, A. II

Crampton, T.

Fisher, F. E

Jewell, $\mathrm{R}$
Mino, $\mathrm{J}$.

Conley, M

Gillette, H

Groth, L. A

Locke, S. D

Lounsbury, J. A

Rosebrugh, A. MI

Sabold, F. II

Scribner, C. E

Scribner, C. E

Scribner, C. E

Scribner, C. E

Virtue, G. F

Young, $H$. $H$

Dressler, C. E

Johnston, J. G

Roberts, I. L

Roberts, I. L

Schiffmann, TI. IV

Iansfield, $\mathbf{F}$

lves, E. B.

Marshall, $N$

Hunt, G. W. and Rich, A. E

Roberts, I. L

Roberts, I. I

Roberts, I. L

Roberts, I. I

Roberts, I. I

Sch reuder, J. G

Schreuder, J. G

Vaughn, J. WV

Arnold, C. R

Dewey, M. WV

Garland, W. H

Merrifield, P. V

Nen, Gr. $S$

Pumpelly, J. K

Pumpelly, J. $\mathrm{K}$

Roberts, I. L

Wightman, M. .

Brady, T. H

Currie, S. C. C

Paiste, H. T

Paiste, H. T

Schmid, A

Batchelder, G. I

Beers, C. B. and Tuttle, W. B.................

Taylor, W. W

Wood, J. J

Dntton, C. E., jr

Eckert, Wr. H. and Gregory, W. H

Fraser, IT.S

Henderson, W. B

Marshall, N

Poberts, I. I

Casper, L

Giles, J. F

Hopson, R. C

Talmage, C. H

Weyburn, E. D

Gunn, J
Motors

Batteries, galvanic

Batteries, secondary

Batteries, galvanic

Distance instruments

-..-.-do do

Alarns

Telegraphy, lightning arrcsters

Railways, systems, overhead, trolleys ......

Lights, arc, gravity feed, clutch.

Annunciators

Cut-outs, mechanical

Measuring vessels .

Lamps and gas fittings, guards

Indicators

Rail Wy gates, electric

Tanning processes

Recorders

Lights, arc, special features

Anti-induction devices - -.

Railways, systems

Telephones, systems

- - - do

do

Applications, tools and machines

Conductors, underground

Magneto-electric

Batteries, secondary

$$
\text { do }
$$

Iotor

Railways, conductor supports and insulators

Telegraphy, multiplex

Gas fitting

Lights, sockets and keys

Electrolysis

-.---.--do do

Paper making, fablics

Batteries, galranic

Telegraphy, relays and sounder

Railway signals

Telephones, systems

Telegraphy, railway car -..................

Railways, systems-

Connectors

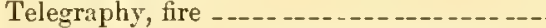

Telegraphy, fire, alarms and cxtinguishers

Batteries, secondary -

Electrolysi

Railways, sytems, circuits, multiple are

Block and tackle.

Galvanometers

Railways, conductor supports and insulators

Lights, incandescent, special features -..--.. do

Elentric locomotives

Drop lights and hangers

Bells

Telegraphy, printing

Meters

Batteries, galvanic

Lighting, systems_.

Lamp shades

Lights, are, gravity feed, gear-clutch

Cut-outs, mechanical

Electrolysis

Indicators

Lights, arc, gravity fced, gear-clutel

Railways, systems, orerhead

Heaters

Applications, tools and machines

Electrolysis

Lights, incandescent, special features

Dec. 2, 1890

Dec. 2,1890

Dec. 2, 1890

Dec. 2,1890

Dec. 2,1890

Dec. 2, 1890

Dec. 2, 1590

Dee. 2, 1890

Dec. 2, 1890

Dec. 2, 1890

Dec. 2, 1890

Dec. 2, 1890

Dec. 2, 1890

Dec. 2, 1890

Dec. 2, 1890

Dec. 9,1890

Dec. 9, 1890

Dec. 9,1890

Dec. 9,1890

Dec. 9, 1890

Dec. 9,1890

Dec. 9,1890

Dec. 9,1890

Dec. 9, 1890

Dec. 9, 1890

Dec. 9, 1890

Dec. 9,1890

Dec. 9,1890

Dec. 9, 1890

Dec. 9, 1890

Dec. 9, 1590

Dec. 9, 1890

Dec. 9, 1890

Dec. 9,1890

Dec. 9,1890

Dec. 9, 1890

Dec. 9,1890

Dec. 9, 1890

Dec. 9, 1890

Dec. 9, 1890

Dec. 9, 1890

Dec. 9,1890

Dec. 9, 1890

Dec. 9, 1890

Dec. 9,1890

Dec. 9,1590

Dec. 9, 1890

Dec. 9,1890

Dec. 9, 1890

Dec. 9,1890

Dec. 9, 1890

Dec. 9,1890

Dec. 9,1890

Dec. 9, 1890

Dec. 9, 1890

Dec. 9,1890

Dec. 9, 1890

Dec. 9, 1890

Dec. 9,1890

Dec. 9, 1890

Dec. 9, 1890

Dec. 9,1890

Dec. 9, 1890

Dec. 9, 1890 


\begin{tabular}{|c|c|c|c|}
\hline No. & & CIASS. & E. \\
\hline & & $15-$ & Jee. \\
\hline & $3=-2$ & Lights, incandescent, special & Dec. 16,1890 \\
\hline & $\mathrm{W}_{-}$ & Meters ......... & Dee. 16,1890 \\
\hline & ith, J. R. and Childs, W-- & ems, automatic ............. & Dec. 16,1590 \\
\hline & phenson, J & - n- & Dee. 16,1890 \\
\hline & - & ial features....... & Dec. 16,1890 \\
\hline & ess, $N_{n} \ldots$ & 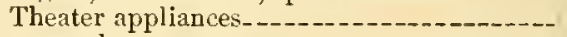 & Iec. 16,1890 \\
\hline & $\mathrm{N}^{\mathrm{T}}-\mathrm{n}-\mathrm{n}$ & $-\mathrm{do}_{-}-\mathrm{C}_{-1}$ & Dec. 16,1890 \\
\hline & $\ldots$ & hones, $\mathrm{m}$ & Dec. 16,1890 \\
\hline & - & $--\ldots--1$ & Dec. 16,1890 \\
\hline & d. G. and Graham & $y$, ci & Dec. 16,1890 \\
\hline & A., jr & ical $\ldots \ldots$ & Dec. 16,1890 \\
\hline & 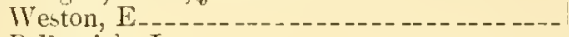 & 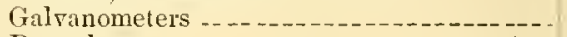 & Dec. 16,1890 \\
\hline & h, $\mathbf{J}_{--n-\cdots}$ & $-\cdots--$ & Dec. 16,1890 \\
\hline & J & -+ & Dec. 16,1890 \\
\hline & S $-\ldots-\ldots$ & -- & Dec. 16,1890 \\
\hline & N. $\mathrm{H}=-$ & - & Dec. 16,1890 \\
\hline & J & -1 & Dec. 16,1890 \\
\hline & $\mathrm{J}=$ & - & Dec. 16,1890 \\
\hline & $\mathrm{t}, \mathrm{H} . \mathrm{J} \ldots \ldots$ & -1 & Dec. 16,1890 \\
\hline & it, H. J & - do.--- & Dec. 16,1890 \\
\hline & t, H. J $\ldots \ldots$ & 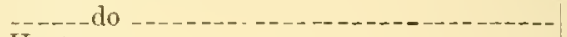 & Dec. 16,1890 \\
\hline & n, E_- & 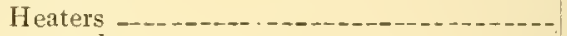 & Dec. 16,1890 \\
\hline & E--- & $-\ldots$ & Dee. 16,1890 \\
\hline & -.-- & 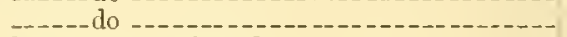 & Dec. 16,1890 \\
\hline & Г. and Fuhrmau, L. P & -- & Dec. 16,1890 \\
\hline & ald, D. G. and Hough, A. H & ---- & Dec. 16,1890 \\
\hline & J & ls & Dec. 16,1890 \\
\hline & 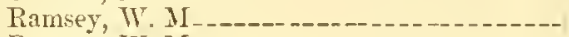 & 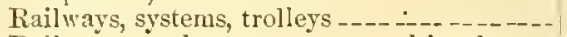 & Dec. 1 \\
\hline & T. MI & ts and insulators_- & Dec. 1 \\
\hline & ooele, C. J ... & - - - - - - - & Dec. 16,1890 \\
\hline & G. $S^{3} \ldots$. & es n-n-no- & Dec. 16,1890 \\
\hline & S - . & lo - - & Dec. 16,1890 \\
\hline & S $\ldots$ & --- & Dec. 16,1890 \\
\hline & N. D. C - & -- & Dec. 16,1890 \\
\hline & ht, D. G. and Potter, J.S. & cent, si & Dec. 1 \\
\hline & and Munn, S. J -.. & S - - - - 1 & Dec. 1 \\
\hline & 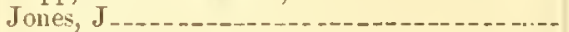 & as, overhead._._. & Dec. 1 \\
\hline & M & s, closed conduit _.......... & Dee. 23,1890 \\
\hline & & hangers_-___._. & Dec: ? \\
\hline & I & nting -. & Dec. 2 \\
\hline & sick, & -.. & Dac. 23,1890 \\
\hline & ick, S. V. I - & do $\ldots . .-$ & Dec. 23,1890 \\
\hline & S. T. B .- & do & Dec. 23,1890 \\
\hline & J. L - & iones, s & Dec. \\
\hline & $-1-n$ & signals _....... & Dec. 2 \\
\hline & P $\ldots$ & systems__...... & Dec. 23,1890 \\
\hline & & gnals.....- & Dec. 23,1890 \\
\hline & {$[-$} & - - - - - & Dec. 23,1890 \\
\hline & Le $\mathrm{R}-\ldots$ & tor supports and insulators. & Dec. 23,1890 \\
\hline & H. A. and Duncan, L - & lary, applications _._._..... & Dcc. 23,1890 \\
\hline & $\mathrm{J}$ & 15 , fire & Dec. 23,1890 \\
\hline & 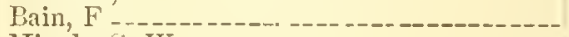 & 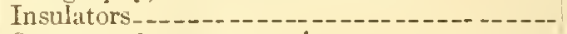 & Dec. 23,1890 \\
\hline & $Y_{-}$ & agnetic_-_-_. & Dec. 2 \\
\hline & J -- & s, galvanic _._........ & Dec. 23,1890 \\
\hline & $11-$ & & Dec. 23,1890 \\
\hline & $\mathrm{P}$ & cal, bou & Dec. 23,1890 \\
\hline & & es -.. & Dec. 23,1890 \\
\hline & $W_{--}$ & $\mathrm{Pa}$ & Dec. 23,1890 \\
\hline & G. P & & Dec. 23,1890 \\
\hline & $\mathrm{H}_{--}$ & Ider & Dec. 2 \\
\hline & C. F & eral & Dec. \\
\hline & H. E. and Grosveno & ns, tools and & Dec. 2 \\
\hline & $\mathrm{er}, \mathrm{C} \ldots \ldots$ & clocks _........ & Dec. 23,1890 \\
\hline & $3 \ldots$ & Rer & Dec. 23,1890 \\
\hline & WI & ransmitters & Dec. 23,1890 \\
\hline & right, D. J & kets and key & Dec. 23,1890 \\
\hline & , C. P. and Hess, J. L. & electric & Dec. 23,1890 \\
\hline & (1) & - - - & Dec. 23,1890 \\
\hline & MI & nduit -- & $\mathrm{De}$ \\
\hline & M & overhead, trolle & Dec. 2 \\
\hline & fo-n & , secondary -.. & Dec. 23,1890 \\
\hline & 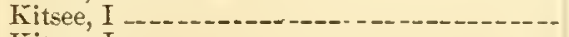 & $10 \ldots$ & Dec. 23,1590 \\
\hline & I & do.--- & Dec. 23,1890 \\
\hline & $\bar{I}$ & - & Dec. 23,1890 \\
\hline & & $g$ and an & Dec. 23,1890 \\
\hline & & Cash indicators.... & Dec. 23,1890 \\
\hline
\end{tabular}




So. YAME.

$\pm 43,47$

$143, \pm 93$

$4+3,+94$

$443, \pm 97$

443,508

$\pm 13,5=0$

443,527

$413, \overline{3} 30$

443,536

443,051

$413, \overline{5}$ อิ

$143, \overline{0} \overline{0} \overline{7}$

413,962

$4 \frac{1}{2}, 3,56$ :

43,56 .

443,580

43,595

$4+3,606$

$\pm \pm 3,632$

443,633

$+13,636$

$+13,6 t^{2}$

$\pm 13,6 \pm 6$

443,661

$\pm 13,67$

$\$ 13,6-1$

413,677

433,693

$43,716^{\circ}$

$+13.21$

$443, i 2$

$413,-20$

$4+3,-27$

$4 \pm 3,-2 ?$

$+13,730$

$\pm 43,-31$

443,732

$4+3,73$

$413,7 \pm 6$

43,748

413,749

43,772

48,787

443,788

143,789

443,790

$14.3 .81 \frac{1}{2}$

443,523

$43,32 \pm$

443,831

$4 \pm 3,9 \pm 1$

143.976

413,950

433,991

$\frac{1}{4} 43,992$

414,005

$4 \pm$, 006

414,067

144,104

411,103

44,110

$111,1 \pm 4$

444,154

144,169

$414,18 \mathrm{~s}$

111,217

414,2 으?

$\pm 1+245$

414,260

$414,2-3$

$+11,254$

411,285

$4 \pm 1,2.96$

414,287

414,265

411,317

44,330
Fears, $F . T$, and Speicher, J

Bergman, $\mathrm{S}$

Bergmann, $\mathrm{S}$

Bremer, W. G

Emmet, II. Le R

Jones, F. IV

Lieb, C. A

Norman, T. II

Stillman, F. H

Wardwell, H. H

IThitcomb, A. II

Bergmann, $\mathrm{S}$

Bergmann,

Bremer,

Langstaffe, $J$

Johnson, E. H

Heymann, E. and F. W

Hermann, E. and F. W

Faure, C. A

Elieson, C. P -

Kilbourn, W. H

Eickemeyer, $\mathrm{R}$

Goldschmidt, P

Hunter, R. M

Wood, J. J

O'Meara, J

Ongley, C. E

Ongles, C. E

Ongles, C. E

Ongler, C. E

Steele, J. W

Steele, J. II

Steele, J. II

Steele, J. IW

Applevard, A. E

Dempster, J. T

Dewer, M. IV

Kells, C. E., j

Ongler, C. E

Ongler, C. E

Ongles, C. E

Ongley, C. E

Berne, A. W

Cope, T. J

Cope, T. J

Luthe, J. TF. and Jeavons, A. E

Manning, J. A

Tan Depoele, C. J.

Ellis, S. R

Hart, II. II

Mason, D

Mason, D

Holcombe, A. G

Reppy, A. II

Farnham, I. H

Randall, B. L

Wilson, J. C

skinner, J. .J

Fuller, L. K

Fan Depoele, C. J.

Fiske, B. A

Payne, C. $\mathrm{Q}$

Hormel, A -

Pentlev, E. II

Cliff, E

Warth, A

Warth, A

Warth, A

Fuller, I. A

Potter, J. S. and Cartwright, D. J

Chase, J. S. add Cartwright, D. J -.--
Class.

DATE.

Telegraphy, fire

Telephones, magnctic

Telephones, electric

Cut-outs, thermal

Railways, conductor supports and insulators

Telephones, electric

Lighting, systems

I,ights, sockets and keys

Wire...

Lead pipe making

Batteries, secondary

Lights, arc, gravity feed, gear-clutch

Lights, sockets, and keys

Shade supports

Cut-outs, thermal

Medical and surgical, batlis

Incrôstation

Lighting, systems.

Lights, are, gravity feed, clutch

ireter

----odo

Applications, tools and machines

Car brakes

Electric locomotives.

Cordage, warp, stop motion

Electric locomotives

Cut-outs, mechanical

Valre-controllers

Telegraphy, fire, alarms and extinguishers -

Telegraphy, fire

Telegraphy, printing

Lighting, systems -

Railways, automatic signals, danger

Railways, automatic signals, trip

Railway gates, electric

Lights, sockets and keys _..._... Dec. 30, 1890

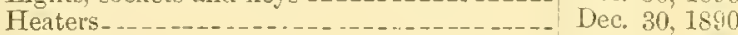

Applications, tools and machines .........- Iec. 30, 1890

Time checks _..... Dec. 30,1890

Dec. 30,1890

Talve controllers ... Dec. 30, 1890

Recorders_... Dec. 30, 1890

Railway signals _._._._................ Dec, 30,1890

Conductors, underground

- do _._..... Dec. 30,1890

Annunciators__._. Dec. 30, 1890

Lights, arc, special features -...-_...-. Dec. 30,1890

Batteries, galvanic -

Motors, alternating ...._..._._._......... Dec. 30 , 1890

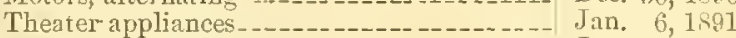

Condnctors, underground _.................. Jall. 6, 189

Railways, conductor supports and insulators_ Jan. 6, 1891

Jin. 6,1891

Magneto-electric -................... Jn, 6, 1891

Lights, arc, gravity feed, gear-clutch _..._-. Jan. 6, 1891

Telephones, systems....._._._._..._._. Jan. 6, 1891

Electric locomotives _._._._._._._._... Jan. 6, 1891

Telegraphy, fire _._._._._._._._._._._. Jan. 6, 1891

Lights, arc, gravity feed, clutch

Music, electric actions................... Jan, 6, 1891

Motors, alternating .......... Jan. 6, 1591

Optics, distance instruments_._._._._. _.... Jan. 6, 1891

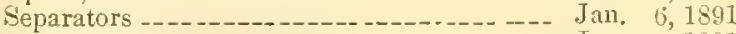

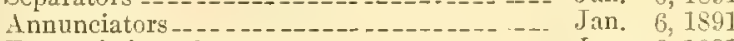

Transmission of power

Drop lights and hangers._........._._._._. Jan, 6, 1891

Mechanical movements

Jan, 6, 1891

do _... Jan. 6, 1891

TTater gages, float

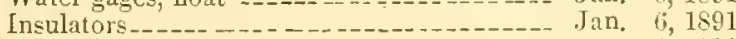

Lights, arc, special features _........ Jan. 6, 1891 


\begin{tabular}{|c|c|c|c|}
\hline No. & NaJre. & CluAss. & DAte. \\
\hline & Jewell, $R_{-}$ & Registers & Jan. 6,189 \\
\hline & Tilson, C. E & Cut-outs, meehani & Jan. 6,1891 \\
\hline & Cutter, G & Conductors, undergrouud & Jan. 6,1891 \\
\hline & S. C. ( & Batteries, secondary - & Jan. 6,1891 \\
\hline & Hunter, R. M & Railways, systems, orerhead, trolleys & Jan. 6,1891 \\
\hline & Anthony, $\mathrm{TV}$. & Regulators . . & Jan. 13,1891 \\
\hline & Diwwes, $\mathrm{A}$ & Drop lights and han & Jan 13,1891 \\
\hline & er, E. & Clocks, electric _-.....-- & Jan. 13,1891 \\
\hline & 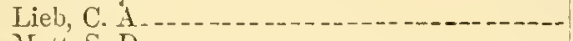 & Railways, syst & Jan. 13,1891 \\
\hline & $-\cdots+\cdots$ & Indicators - & Jan. 13,1891 \\
\hline & $\mathrm{D}$ & $----d$ do --- & Jan. 13,1891 \\
\hline & $\begin{array}{l}\text { S. D } \\
\text { T. C. }\end{array}$ & - - - - - & Jau. 13,1891 \\
\hline & T. C. & Ligh & Jan. 13 \\
\hline & $\mathrm{B}_{-\ldots}$ & clutch & Jan. 1 \\
\hline & B. B & $\mathrm{Lig}$ & Jan. 13,1891 \\
\hline & A. $P$ & $\mathrm{Con}$ & Jan. 13,1891 \\
\hline & E. 1 & Eles & Jan. 13,1891 \\
\hline & -- & & Jan. 13,1891 \\
\hline & ley, W. IV & Cl & Jan. 1 \\
\hline & J. $B_{-}$ & & Jan. 1 \\
\hline & dell, M. a & & Jau. 13,1891 \\
\hline & $\mathrm{D}$ T. A & Lig & Jan. 13,1891 \\
\hline & - & H & Jan. 1 \\
\hline & & G & Jan. 131891 \\
\hline & & Sy & Jan. 13,1891 \\
\hline & & & Jan. 1 \\
\hline & -1 & --- & Jan. 13 \\
\hline & & & Jan. 1 \\
\hline & I & & Jan. \\
\hline & 0 & 1 & Jan. \\
\hline & & & Jan. \\
\hline & O & A & Jan. \\
\hline & E - & & Jan. \\
\hline & J. & $\mathrm{R}$ & Jan. \\
\hline & W. & & Jan. 13, \\
\hline & $\mathrm{I}$ & & Jan. 1 \\
\hline & & & Jan. \\
\hline & & II & Jan. 13,1891 \\
\hline & & & Jan. 13,1591 \\
\hline & I & & Jan. 13,1891 \\
\hline & I & & Jan. 13, \\
\hline & & Ma & Jan. \\
\hline & $y$ & Gal & Jan. 13,1891 \\
\hline & $y$ & do - & Jan. 13,1 \\
\hline & & do - & Jan. 13,18 \\
\hline & $\mathrm{H}$ & & Jan, 20 \\
\hline & & & Jau. 20,18 : \\
\hline & & $\mathrm{R}$ & Jan. 20,1 \\
\hline & & & Jan. 20,1 \\
\hline & F. & li & Jan. 20,189 \\
\hline & $I$ & & Ja \\
\hline & & $\mathrm{E}$ & 20,18 \\
\hline & & C & Ja \\
\hline & & $\mathrm{F}$ & $J$ \\
\hline & & de & \\
\hline & & & $\mathrm{J}$ \\
\hline & G. I & $\mathrm{H}$ & $=0,18$ \\
\hline & $.1 T^{r}$ & c los & Jan. $-0,10$ \\
\hline & & & Jar \\
\hline & & Heaters - & $18:$ \\
\hline & & & $\mathrm{J}:$ \\
\hline & & do & 20,18 \\
\hline & & $\mathrm{s}, \mathrm{tl}$ & \\
\hline & $\begin{array}{l}1 \\
T\end{array}$ & MI & \\
\hline & $\begin{array}{l}\mathrm{T} \\
\mathrm{B}\end{array}$ & & J \\
\hline & & & \\
\hline & & & 0,1 \\
\hline & & & \\
\hline & & & \\
\hline & & B & 20,15 \\
\hline & & & \\
\hline & & $\mathrm{F}$ & $J a n .20,189$ \\
\hline & & $\begin{array}{l}\mathrm{C} \\
\mathrm{H}\end{array}$ & Jan. 20,189 \\
\hline & $\begin{array}{l}\mathrm{P} \\
\mathrm{T} . \mathrm{X}\end{array}$ & & Ja \\
\hline & .1 & & $\operatorname{Jan}$ \\
\hline & & ml-i & Jan \\
\hline
\end{tabular}




\begin{tabular}{|c|c|}
\hline So. & Name. \\
\hline & Toun \\
\hline $\begin{array}{ll}103 \\
106\end{array}$ & $\begin{array}{l}\text { Iogler, W. } \\
\text { Wilev, E. C }\end{array}$ \\
\hline 113 & Hörenz, $\mathbf{F}$. \\
\hline 136 & Elkins, W. H \\
\hline $11: 2$ & Hughes, J. S \\
\hline 14 & Hunter, R. II --. \\
\hline 146 & - \\
\hline 161 & - - - - - - - - - - - - \\
\hline $4 \frac{1}{2}, 176$ & Hayes, J. A \\
\hline $\begin{array}{l}415,207 \\
415,217\end{array}$ & 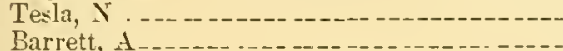 \\
\hline 100 & - \\
\hline 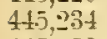 & - n \\
\hline- & Potter, J. S \\
\hline $45,-259$ & Curtis, W. B. and Himrod, C \\
\hline & 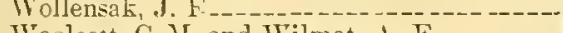 \\
\hline
\end{tabular}

Woolcost, C. .I. and Wilmot, A. E

Cutter, G. and Armstrong, C. G

Lenlart, P. F

145,311

Striemer, A

Sprinkmanu, F

Bergmann, $s$

415,362

Bergmann, $s$

Bergmann, $\mathrm{S}$

Bergmann. s

Brusb, C. F

Crowdus, TI. At

Doggett, J

Holmes, J.

Norieza, E

Short, S. H

Bromn, A. H

Dresshell, TI. II

Sprague, F. J.

Condict, G. H -

Burrell, S. J. and Markland, VT. H.-

Cheswright, H. T

Crane, M. G.

Easton, J.

Fitcli, G

Loy. I. W. and $\mathrm{O}^{\circ}$ Toole, $\mathrm{R}$

Zipernowsky, C

Foster, C. and Bevis, W. H

Patten, F. J

Patten, F. J

Bentler, E. II

Chambers, J.

Stitzel, $\mathrm{F}$, and TTeinedel, $\mathrm{C}$

Stitzel, F. and

Hunter, R. II

Bottome, T. D

Bottome, T. D

Bottrme, T. D

Etheridge, $\mathrm{H}$

Stevens, B. A

White, O. C

Cutler, H. H

Cutler, H. H

TVeller, F. A

Hoffman, J. W

Hoffman, J. W

Dewey, II. W

Ely, E. S

Winton, $\mathrm{H}$.

Cole, F. W

Cole, F. IT

Cole, F. W

Cole, F. W

Cole, F. W

Cole, F. WV

Cole, F. W

Class.

DATE.

Railways, conductor supports and insulators_. Jan. 20, 1891

Railway signals _..._._._._-_._._._._-_- Jan. 20, 1891

Time checks _..._._._._._._._._. Jan. 20, 1891

Lights, arc, gravity feed, clutch.......... Jan. 20, 1891

Railways, conductor supports and insulators_ Jan. 20, 1891

Electric locomotives _..._................... Ja. 20, 1891

Cut-outs, mechanical _._._._._._._._._. Jan. 20, 1891

Printing, hand stamps ---_--_-_-_........ Jan. 27, 1891

Lights, are, positive feed, gear-clutcl _..__- Jan. 27, 1891

Wotors, alternating ................ Jan. 27, 1891

Cut-outs, thermal _._._.................. Jan. 27, 1891

Induction coils _-_-_-___________. Jan. 27,1891

Conductors, anti-iudnctive _._._._._._. Jan. 27, 1891

Lights, incandescent, special features ....... Jan. 27, 1891

Lamp shades.......... Jan. 27, 1891

Bells - - Jan. 2T, 1891

Alitrms -

Cut-outs, mechanical _................ Jan. 27, 1891

Metal founding, wheels _......_._._...... Jan. 27, 1891

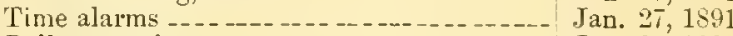

Bniler coverings............................. 27, 1891

Cut-onts, mechanical _....._._._._._..... Jan. 27, 1891

Lights, sockets and keys_................. Jan. 27, 1891

Co do -... Jan. 27, 1891

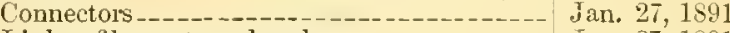

Lights, filaments and carbons _......... Jan. 27, 1891

Railways, systems, orerhead ..._... _...... Jan. 27, 1891

Batteries; secondary _......._._._._._._. Jan. 27, 1891

Batteries, galvanic -...-........... Jan. 27, 1891

Telegraphy, relays and souudcrs _.......... Jan. 27, 1891

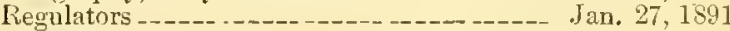

Telephones, electric -........ Jan. 27,1891

Railways, systems _._.

Alarmis -_-_- -

Iotors _._. Jan. 27, 1891

Railways, systems, overhead .............. Jan. 27, 1891

Magneto-electric _..._._._._._._._. 27, 1891

Telcphones, calls_............................ 3, 1891

Batteries, secondary _._._._._._._._._._. Feb. 3, 1891

Telegraphy, fire _..... Feb. 3, 1891

Lights, arc, gravity feed, gear-clutch _._._. Feb. 3, 1891

Drop lights and hangers _._._._._._._._. Feh. 3, 1891

Railway signals - .......... Feb. 3, 1891

Iilectric locomotives_._._._._._._._._. Feb. 3, 1891

Railways, conductor supports and insulators_ Feb. 3, 1891

Motors, alternating _............. Feb. 3, 1891

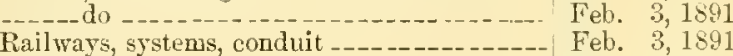

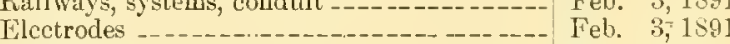

Printing, branding stamps _.._._._._. . Feb. 3, 1891

Railway signals _._._. Feb. 3, 1891

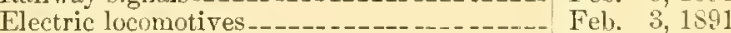

Railways, systems, conduit _... _............ Felo, 3. 1891

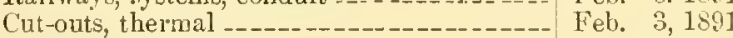

Systems of distribution_._.___._._._._. Feb. 3, 1891

Lights, incandescent _._._._._._._._. Feb. 3, 1891

Telegraphy, automatic -..... Feb. 3, 1891

Telephones, details -..... Feb. 3, 189

Lights, arc, gravity feed, clutch _......... Feh. 3, 1891

Igniting de vices, automatic _..._._._._. Feb. 3, 1891

_-_do _-_._. Feb. 3, 1891

Motors --

Comeetors _._._._._... Feb. 3, 1891

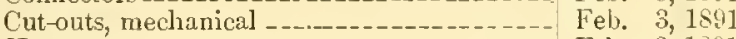

Heaters_... Feb. 3, 1s91

Indicators -

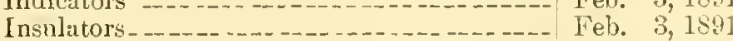

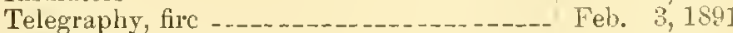

Annunciators -

Telegraphy, repeaters -.................... Feb. 3, 1891

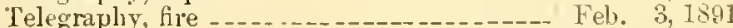

Telegraphy, fire -

-

Feb. 3,189

Feb. 3, 1891 


\begin{tabular}{|c|c|c|c|}
\hline No. & ME. & Class. & DATE. \\
\hline 445,822 & seoth, $T_{0}, \mathrm{~A}$ & Lights, incandescent, special features & Feb. 3,1891 \\
\hline & Short, S. H- & Connectors & Feb. 3,1891 \\
\hline 860 & De Place, L & Applications, general ....... & Feb. 3,1891 \\
\hline & rrett, S. H & Batteries, secondary & Feb. 3,1891 \\
\hline & Schwarze, C. J & Iagneto-electric & Feb. 3,1891 \\
\hline & E. P & Motors & Feb. 3,1891 \\
\hline 88 & Sharp, E. P & Railways, conductor supports and insu & Feb. 3,1891 \\
\hline & Douse, T. R & Telegraphy, circuit closers, thermal _- & Feb. 3,1891 \\
\hline & r, R. MI & Railways, systems, over & Feb. 3,1891 \\
\hline & L. F & Lighting, systems_-- & Feb. 3,1891 \\
\hline & Lean, G. R -- & Cut-outs, mecbanical _...-... & Feb. 3,1891 \\
\hline & Branch, J. R & Insulators. & Feb. 10,1891 \\
\hline & Gaynor, T. F & Annunciators -..... & Feb. 10, 1891 \\
\hline & E. A. & Transmission of power. & Feb. 10,1891 \\
\hline & E. A & Cut-outs, mechanical -. & Feb. 10,1891 \\
\hline & Titus, J. E. & Drop ligbts a & Feb. 10,1891 \\
\hline & E. $\mathrm{K}$. & IIagneto-electric ... & Feb. 10, 1891 \\
\hline & -...-.. & Batteries, secondary ....... & Feb. 10,1891 \\
\hline & . $\mathrm{H}_{-}$ & ive feed & Feb. 10,1891 \\
\hline & $\mathrm{H}$ & -.... do & Feb. 10, 1891 \\
\hline & G. IV & Lightning rods _.... & Feb. 10,1891 \\
\hline & & incandescent, special & Feb. 10, 1891 \\
\hline & Westinghouse, G., jr & Railway signals & Feb. 10, 1891 \\
\hline 74 & O'Meara, J & $\therefore \quad 0 \quad-2$ & Feb. 10,1891 \\
\hline & $\mathbf{J}_{-}$ & Ligh & Feb. 10,1891 \\
\hline & -........... & ..... & Feb. 10,1891 \\
\hline & & --- & Feb. 10, 1891 \\
\hline & B & Telegrapliy, fire, alar & Feb. 10,1891 \\
\hline & $\mathrm{B}$ & Scale bear & Feb. 10,1891 \\
\hline & & $\mathrm{D}$ & Feb. 10,1891 \\
\hline & & undergr & Feb. 10,1891 \\
\hline 29 & . S & $\mathrm{R}$ & Feb. 10,1891 \\
\hline d. & $\mathrm{G}$. & E] & Feb. 10,1891 \\
\hline & $\mathrm{L}$ - & -.. & Feb. 10,1891 \\
\hline & C. & ity feed, gea & Feb. 10, 1 s91 \\
\hline & C. & $\mathrm{Li}$ & Feb. 10, 1891 \\
\hline & C & $\mathrm{R}$ & Feb. 10,1891 \\
\hline 76 & E. 7 & $\mathrm{~K}$ & Feb. 10,1891 \\
\hline & Is $S$ & $\mathrm{R}$ & Fcb. 10 \\
\hline & E. & $\mathrm{R}$ & Feb. 17, 1891 \\
\hline & & Raily & Feb. 17, 1891 \\
\hline 19 & E. & Railwars, srstems, & Feb. 17,1891 \\
\hline & B & _.... do - . & Feb. 17, 1891 \\
\hline & $\mathrm{Br}$ & Telegraphy, multiplex & Feb. 17,1891 \\
\hline & & -ndo - n d & Feb. 17, 1591 \\
\hline 28 & Cahoo & Railways, sy & Feb. 17,1891 \\
\hline & J. A & h & Feb. 17,1591 \\
\hline & & $\mathrm{R}$ & Feb. 17, 1891 \\
\hline & $\mathrm{s}$ & Lights, incindescent, & Feb. 17, 1891 \\
\hline & $\mathrm{E}$ & Railways, cot & Feb. 17, 1891 \\
\hline & $J_{-}$ & Speed iuensures & Feb. 17,1891 \\
\hline & & Galvanometers _..... & Feb. 17,1891 \\
\hline & $\mathrm{T}$ & R & Feb. 17, 1891 \\
\hline & II & Galranometers ...... & Feb. 17, 1891 \\
\hline & & 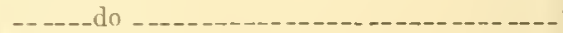 & Feb. 17, 1891 \\
\hline & $\mathrm{E}_{-}$ & do & Feb. 17,1891 \\
\hline & & do & Feb. 17,1891 \\
\hline & 1 & Hiscellaued & Feb. 17, 1891 \\
\hline & C & Maguetic locks and bolt: & Feb. 17, 1891 \\
\hline & & of liotribut: & Feb. 17, 1891 \\
\hline & amis, $I$. & teries, secondary & Feb. 17,1891 \\
\hline & & o-electric & Fel. 17,1891 \\
\hline & Wri & B & Feb. 17 \\
\hline & Cook, W.S. & Railwar sigmals. & Feb. 17, 1591 \\
\hline & $\mathrm{Fe} C \mathrm{C}-\mathrm{s}$ & Lights, are, special feat & Feb. 17,1581 \\
\hline & $\mathrm{Ra}$ & s, srstems, batt & Feb. 17,1891 \\
\hline & & Telephones, calls & Feb. 17,1891 \\
\hline & & mechanical & Feb. 1 \\
\hline & & Annunciators & Feb. 17, 1891 \\
\hline & & Conductors & Feb. 17, 1891 \\
\hline & IT. H & Lights, filaments and carbons & Feb. 17, 1891 \\
\hline & & II & Feb. 17,1891 \\
\hline & Edi & Ligliting, systems, secondary battery & Feb. 17, 1891 \\
\hline & $\mathrm{E}$ & Electric locomotires & Feb. 17, 1891 \\
\hline & son, T. A & Lights, arc, gravity fe & Feb. 17,1841 \\
\hline & & $\mathrm{L}$ & Feb. 17, 1891 \\
\hline & seț, C. A 1 - & Wusic, electric actions & Feb. 17, 1891 \\
\hline
\end{tabular}


No.

44,703

416,704

$\pm 1+, i 12$

411,727

$+46,730$

$416,72=$

446,76

$\pm 6,817$

$\pm 46,8=2$

$446,8 \div 20^{2}$

$\pm 16,831$

$+46,833$

$\pm 46.83 \pm$

$4+6,846$

$\pm 46,864$

446,571

146,$8 ;$

$116,89.5$

$446,90^{\circ}=$

146,907

$\pm 46,917$

$\$ 16.931$

$446,93.5$

$\$ 16,966$

416,967

$\pm 46,9- \pm$

46.985

$+46,990$

47,016

447,023

44,045

$44 \overline{7}, 0 . \overline{8}$

$4 \overline{7}, 0.59$

$47,00^{\circ} 0$

47,031

$44 \overline{7}, 062$

$+47,07$

$4 \bar{i}, 104$

$4 \overline{7}, 10 \overline{3}$

$4 \overline{3}, 127$

$+47,134$

47,135

$\pm \pm 7,1 \pm 3$

41:,166

$44 \bar{\gamma}, 17 \%$

$\$ 4 \overline{7}, 194$

447,195

$\pm 17,198$

$44 \bar{i}, 208$

$+47,215$

$+47,200$

$44 \overline{7}, 230$

$44 \overline{1}, 25 \overline{-}$

447,256

447,279

$4 \overline{1}, 28.3$

14,291

$4+7,31$.

$+1 \bar{i}, 321$

$+47,392$

47,338

$+47,3.50$

447,352

447,353

47,393

47,384

$47 \overline{7}, 385$

$+47,409$

47,426

447,449

47,469

447,452

$147,48.5$

447,486

447,494

147,495
Name.

Class.

DATE.

Dewey, M. II

Edwards, W. C

Rice, D. H

Blair, J. and Hunter, A. G

Brown, F. H

Currier, J. B. and Rice, D. H

Buchanan, C. Y

Tan de Plancke, D. and G

Hunter, R. II

Boynton, E. M

II irt, H. C

Donovin, T. J

Honter, R. iL

Hunter, R. MI

Barron, A. M

Gutmann, L

Lieb, C. A -

Madclen, A. F

Seymour, A. P

Tirrel1, J. P

Whitlock, E

Harver, E. T

Short, $\mathrm{H}$

smith, R. T

Field, S. D

Flowers, M. I

Jemp, H. and Schmidt, L. II

Mackloskie, C. II. and Baker, W. E

Orfurd, J. M

Fobinson, MI. W

Carpenter, C. F

Ginochio, N. B

Nores, B. J

Nores, B. J

Tryes, B. J

Nopes, B. J

Noyes, B. J

Kirnan, W. H

Dewey, M. II

Dudley, W. J

Butterfield, H. R

Lee, H. and Lebrun, E

Lee, $\mathrm{H}$ -

Ashbaugh, J. I

smith, S. II

Edwards, T. M

Mercadier, E. J. P

Willer, J. E. A

Parker, J. A

Van Depoele, C. J

White, A. C

Chamberlain, J.

Wower, S. E

Yickerson, $\mathrm{W}$. E. and Berrenberg, A

Currie, S. C. C

Hunter, R. II

Blades, H. H

Gutman, H. J

Hargra re, T. C

Knight, W. H

Medbery, H. J

Rice, E. W. jr

Rich, C. C-

Thomson, E

Van Hoesen, G. D

Kaiser, J. H. and Ledwith, A. B

Gould, C. C

Hargrave, $\mathrm{T}$.

Main, W

Ward, B. B

Beach, F. G

o'leara, J

Short, S. HI
Applications, general

Telephones, calls

Batteries, galvanic

Inductoriums, induction coils

Telephones calls

Separators

Clocks, electric

Railways, systems, battery

Electric lıcomutices.

Lights, incandescent, special features

Heaters

Riliways, systems, overhead

Railways, systems, eireuits, multiple are

Telegraphy, circuit closers, thermal

Hotors, plternating

Connectors .

Miscellaneous compositions

Cut-outs, thermal

Cut-uuts, electro-magnetic

Elerators

Kuilway signals

Ruilways, systems, overhead, trolleys

Car brakes

Cint-outs, thermal

Magnetic lucks and bolts

Heaters

Fuilwars, conductor supports and insulators

Lights, sockets and keys

Batteries, galvanic

Heaters

Telegraphy, fire

-1
$-2-1$

Clocks, electric

Henters

Priuting, matrix-making - do

Heaters

Telegraphy, transmitters

Lighting, systems

Telephones, magnetic

Annunciators .

Telegraphy, reed

Railways, conductor supports and insulators

Railways, systems, converter

Telephones, systems

Railways, systems, battery

Electric locomotives

Lighits, incandescent

Batteries, secondary

Railways, systems, overhead

Motors

Lights, sockets and keys

Printing, matrix-making

Railways, systems, conduit

Conductors, underground

Magneto-electric

Heaters -

Lights, arc, gravity feed, clutch

Magneto-electric -..--.-.--

Sack scales

Magnetic locks and bolts .

Telephones, systems.

Printing, matrix-making

Magneto-electric

Lighting, systems

Telephones, systems

Lighting, systems

Ratways, conductor supports and insulators.

Feb. 17, 1891

Feb. 17, 1891

Feb. 17, 1891

lieb. 17,1891

Feb. 17, 1891

Feb. 17, 1891

Fel, 17, 1591

Feb. 17, 1891

Fcb. 17, 1891

Feb. 17, 1891

fieb. 17, 1891

Feb. 17, 1891

Feb. 17, 1891

Feb. 17, 1891

Fel. 24, 1891

Fub. 21, 1891

Feb. 24, 1891

Feb. 24, 1891

Fel. 24, 1891

Fel. 2t, is91

Feb. 24, 1891

Feb. 24, 1891

Feb. 24, 1891

Fel, 24, 1891

Fel. 24, 1891

Fel). 24, 1891

Fel. 24, 1891

Fcb. 24, 1891

Fel. 24, 1891

Feb. 21, 1891

Fcb. 21, 1891

Fub. 24, 1891

Feb. 24, 1891

Feb. 24, 1891

Feb. 2t, 1891

Feb. 24, 1891

Feb. 24, 1891

Feb. 2t, 1891

Fel, 24,1891

Feb. 24, 1891

Feb. 24, 1891

Feb. 24, 1891

Feb. 24, 1891

Feb. 21, 189

Feb. 24, 1891

Feb. 24, 1891

Feb. 24, 1891

Feb. 24, 1891

Feb. 24,1891

Feb, 24 189

Feb. 24, 15?1

Feb. 24, 1891

Feb. 24, 1891

Feb. 24, 1891

Feb. 24, 1891

Fel. 24, 1891

Feb. 24, 1891

Mar. 3, 1891

Mar. 3, 1891

Mar. 3, 1891

Mar. 3, 1891

Mar. 3, 1891

Mar. 3, 1891

Mar. 3, 1891

Mar. 3, 1891

Mar. 3, 1891

Mar. 3, 1891 


\begin{tabular}{|c|c|c|c|}
\hline No. & IE. & ClaAss. & DATE. \\
\hline 05 & Coburn, L- & Sliding donrs ... & \\
\hline & Taintor, G.. & Cintouts, electro-magnetic...... & Mar. 3,1891 \\
\hline & Cram, W. E & Annunciators - & Mar. 3,1891 \\
\hline & Kennedy, R. & Indnction coils & Mar. 3, 1891 \\
\hline & Schroeder, G. & Electrolysis _....... & Mar. 3, 1891 \\
\hline & Williauns, H. I & Recorders -....... & Mar. 3,1891 \\
\hline & ll, W. P _- & Railways, systems, over & Mar. 3, 1891 \\
\hline & ion, G. C & magnetic_........ & Mar. 3, 1891 \\
\hline & -.....- & Lights, filuments and carbons & Mar. 3, 1891 \\
\hline & & 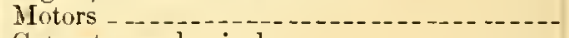 & Mar. 3, 1891 \\
\hline & G. W & uts, mechanical & Mar. 3, 1891 \\
\hline & op, F__.... & Mutors & Mar. 3, 1891 \\
\hline & 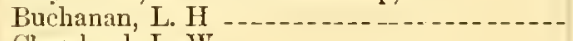 & gear-clutch. & Mar. 3,1391 \\
\hline & -......- & Telegraphy, circuit closers & Mar. 3,1891 \\
\hline & - ...-.... & ity feed, clutch & Mar. 10, 1891 \\
\hline & -........ & -...... & Mar. 10, 1891 \\
\hline & $\mathrm{C}$ & hical _ - & Mar. 10, 1891 \\
\hline & (n) & 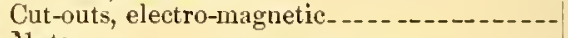 & Mar. 10,1891 \\
\hline & -.--.- & 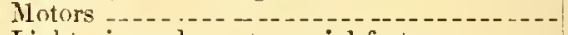 & Mar. 10, 1891 \\
\hline & & special feature & Mar. 10, 1891 \\
\hline & ----- & 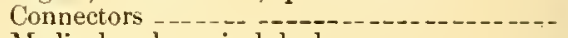 & Mar. 10,1891 \\
\hline & 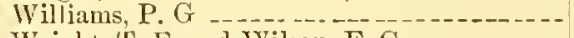 & d surgical, body-wear & 10,1891 \\
\hline & E. G-....... &..--2. & $M r$ \\
\hline & 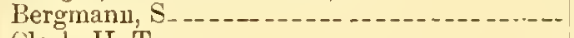 & mechanical _ & 1891 \\
\hline & - - - - - - & n-n & Mar. 10,1891 \\
\hline & & $\mathrm{T}$ & 10,1891 \\
\hline & 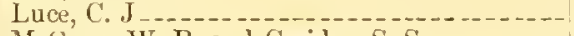 & rhead.......-. & 1891 \\
\hline & S. $S \ldots \ldots$ & y, fire & Mar. 10, 1891 \\
\hline & --n-n-n & S-- & Mar. 10, 1891 \\
\hline & $-1-n$ & 0 & 10,1891 \\
\hline & n-n & - & 10,1891 \\
\hline & - & (n) & $\mathrm{MI}$ \\
\hline & 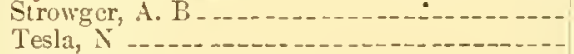 & ems, automatic -... & Mar. 10,1891 \\
\hline & $-\cdots$ & US_-nen & Mar. 1 \\
\hline & -n.......... & o-electric -_...- & $\mathrm{Ma}$ \\
\hline 39 & 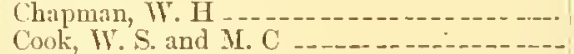 & $2-1-2-1-2-1$ & Mar. 1 \\
\hline & 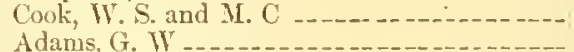 & --- & Mi: \\
\hline & - - - - - - & and extingu & $\mathrm{Ml}:$ \\
\hline & - & $-\cdots-$ & M: \\
\hline & - & bỉock systems ..... & $\mathrm{Ma}$ \\
\hline & - & $-\cdots+\cdots$ & 891 \\
\hline & - - & $\mathrm{T}$ & 1891 \\
\hline & -1 & & 1892 \\
\hline & $-\cdots$ & gear-clntch_ & Mi: \\
\hline & & is and bolts ............ & 1891 \\
\hline & L. 1 & ....- & 1891 \\
\hline & b, G. F & body-wear -.- & M \\
\hline & C $\mathrm{C}$ & 1 repeaters - -- & 891 \\
\hline & ckson, G. F - & 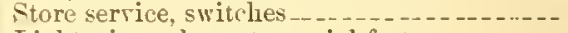 & $\mathrm{MI}$ \\
\hline & & t, special features & 1891 \\
\hline & & gnetic_-_-_-_-_- & $\mathrm{M}$ \\
\hline & $-\cdots$ & ------- & $\mathrm{M}_{\mathrm{s}}$ \\
\hline & & 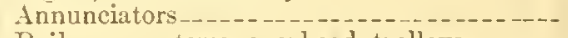 & M \\
\hline & & , overhead, trolley & $\mathbf{M}_{\mathrm{s}} \mathrm{rat}$ \\
\hline & & 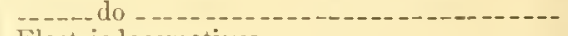 & M \\
\hline & I & & \\
\hline & & pports and ins & $\mathrm{M}$ \\
\hline & & comotives _............. & $\mathrm{Ma}$ \\
\hline & 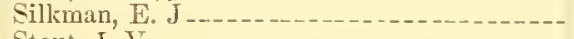 & 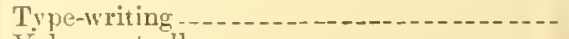 & 591 \\
\hline & & 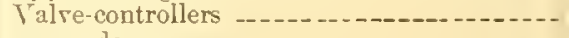 & 1891 \\
\hline & & 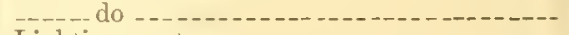 & 1891 \\
\hline & & & 891 \\
\hline & & & 891 \\
\hline & & & \\
\hline & & couplings ... & $M$ \\
\hline & & signals _...... & $\mathrm{Me}$ \\
\hline & & & \\
\hline & & & 1891 \\
\hline & & & \\
\hline & & ing --.- & $\mathrm{M}_{\mathrm{i}}$ \\
\hline & & 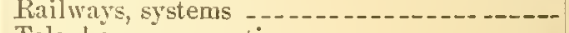 & $\mathrm{Ma}$ \\
\hline & & gnetic - - - & \\
\hline & & & \\
\hline & $\mathrm{Is}, \mathrm{O} . \mathrm{P}$ & & \\
\hline & & & \\
\hline 65 & & & Mar. 1\%, 189 \\
\hline & & & \\
\hline
\end{tabular}




No. NAME. Class. Date.

45,369

448,378

$44,39$.

418,398

44,399

448,400

448,401

448,404

448,418

48,431

448,461

$\pm \$ 8,468$

$4+8,503$

448,523

445,940

448,341

448,561

44,562

448,363

448,587

448,594

448,598

448,603

448,604

448,618

448,62

$4\{8,644$

418,653

448,666

41,669

448,676

448,677

448,67

48,680

413,681

448,682

448,684

$4.48,698$

445,703

448,711

418,712

448,726

$448,7+1$

$418,74:$

418,751

448,778

448,79

44, $78:$

448,792

448,798

48,831

445,830

448,840

448,847

$448,8.36$

448,86 .

448,879

443,894

448,910

448,91

448,915

448,916

448,920

448,927

48,936

448,95 .

448,956

443,930

443,98 .

418,994

448,999
Regan, D. S

oden, F. H

Wheeler, G. K

Wilson, J. C

W'ilson, J. C-

IVilson, J. C

Amstutz, X. S

Easton, J. IT

Johnson, E. H

Sterart, R.A A

Crow, B. F

Hunter, R. M

McGénniss, D

Parker, T. and Robinson, A. E-

Van Depoele, C. J

Tan Depoele, C. J

Ian Depoele, C. J

Redfield, C. L

Sinith, C. R

Wheeler, S. S. and Bradley, C. S

Chaplin, E. D

Conner, II. A

Hunter, R. M

Benjamin, G. V

Farmer, MI. G

Hunter, K.-M

Nutting, S. E

Perret, F.A.

Shallenberger, $\mathrm{O}$. $\mathrm{B}$

Shallenberger, O. B

Shlaudeman, F .

Shlaudeman, F

Short, S. H

Short, S. H

Singer, IV. F

Turner, D. B

Wirt, H. C

Farnham, I. H

Gallagher, J. J

Oeÿan, $T$

Mueller, E. T.

OMeara, J

Kinsman, F. E

Erlison, T. A

Edison, T. A

Hunter, R. II

livirt, C

Hirlimann, C. J

Eickemeyer, P

Pocock, F. A

Short, S. H

Leakin, J. H

Graff, E. D

Smith, C. G

Dewey, M. IV

Thomson, E

Conradson, C. M

Er]wein, G. and Heller, F. G. A

Erlwein, $G$

Erlwein, Gr

Heller, F. G. A

Peacock, R. and Lange, H. L

Collins, J. J

Fuller, IV. C

Graham, G. H. and Gaunane, T.

Miller, I. N

Narjot, E,

Redfield, C. I

Scales, WT. SS

cales, II.S

Shallenberger, $\mathrm{O} . \mathrm{B}$

shallenberger, $\mathrm{O}$. B

Shallenberger, O. B

Capek, J. V

Capek, J. V
Air and gas engines, explosive.

Indicators.

Insulators

Telegrapliy, fire

$$
\text { do }
$$

Thermostat

Engraving machines

Lights, arc, gravity feed, gear-clutch

Cut-outs, mechanical

Railways, systems, conduit ...........

Lights, incandescent, special features.

Railways, systems, overhead

Railways, systems, circuits, multiple arc --

Metal, curved surfaces

Electrolysis

Electric locomotives

-. do

Printing, matrix-making

Wire

Railways, systems

Motors

Conductors

Railways, systems, overhead, trolleys ......

Ear trumpets.

Inductoriums, transformers, stationary

Railways, systems, circuits, series --.

Magneto-electric

Meters do

Lights, sockets and keys

Connectors

Regulators

Rheostats

Telegraphy, fire -

Cut-outs, mechanical

Railways, conductor supports and insulators

Railways, systems, overhead

Alarms

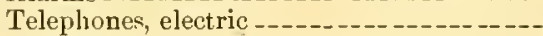

Lamp shades.

Heaters

Railway signals, sectional rail

Railways, systems, circuits, multiple arc -.-

Telegraphy, circuits and systems

Cut-outs, thermal

Galvanometers

Batteries, galvanic

Railways, systems, overhead

Electric locomotives .

Batteries, galvanic

Railway, cab, sectional rail

Drop lights and hangers

Heaters

Meters

Electric locomotives

Lights, filaments and carbons

- -

Electric locomotives

Batteries, galvanic

Merlical and surgical, body-wear-_._._._.-

Insulators

Cut-outs, thermal

Air and gas encines, explosive

Printing, matrix-making

Clocks, electric

Meters

Meters do

do

Heaters

Heaters

Mar. 17, 1891

Mar. 17, 1891

Mar. 17, 1891

Mar. 17, 1891

Mar. 17, 1891

Mar. 17, 1891

Lar. 17, 1891

Mar. 17, 1891

Mar. 17, 1891

Mar. 17, 1891

Mar. 17, 1891

Mar. 17, 1891

Mar. 17, 1891

Mar. 17, 1891

Mar. 17, 1891

Mar. 17, 1891

Mar. 17, 1891

Mar. 17, 1891

Mar. 17, 1891

Mar. 17, 1891

Mar. 17, 1891

Mar. 17, 1891

Mar. 17, 1891

Mar. 17, 1891

Mar. 17, 1891

Mar. 24, 1891

Mar. 24, 1891

Mar. 24, 1891

Mar. 24, 1891

Mar. 24, 1891

Mar. 24, 1891

Mar. 24, 1891

Mar. 24, 1891

Mar. 24, 1891

Mar. 24, 1891

Mar. 24, 1891

Mar. 24, 1891

Mar. 24, 1591

Mar. 24, 1891

Mar. 24, 1891

Nar. 24, 1891

Mar. 24, 1891

Mar. 24, 1891

Mar. 24, 1891

Mar. 24, 1891

Mar. 24, 1891

Mar. 24, 1891

Mar. 24, 1891

Mar. 24, 1891

Mar. 24, 1891

Mar. 24, 1891

Mar. 24, 1891

Mar. 24, 1891

Mar. 24, 1891

Mar. 24, 1591

Mar. 24, 1891

Mar. 24, 1891

Mar. 24, 1891

Mar. 24, 1891

Mar. 24, 1891

Mar. 24, 1891

Mar. 24, 1891

Nar. 24, 1891

Mal. 24, 1891

Mar. 24, 1891 


\begin{tabular}{l|l|l|l}
\hline No. & Nane. & Class. & Date. \\
\hline
\end{tabular}

449,044

$\$ 49,081$

449,090

449,106

449,108

449,125

419,153

$+49,163$

449,174

449,180

$+49,180$

419,188

449,196

$\pm 49,197$

419,206

$+49209$

449,226

449,227

449,236

$\pm 19,23$

449,235

$4+4.24$

$4+9,2+9$

$449,25$.

$+49,258$

$\pm 49,266$

449,282

149,258
$+19,289$

$+49,299$

449,301

$4+9,302$

$4+9,30$

449,34

449,348

$4+9)$

419,35

$+49,362$

$+49,315$
$+49,400$

$44,+03$

$4 \pm 9,40$.

$+19,40$

449,463

$449, \pm 74$

$449,+90$

$4+9,569$

449,514

449,575

$4+9,5-6$

$\pm 40,604$

44,610

449,611

449,639

449,651

$+49,650$

$\$ 49,660$

449,661

449,662

419,672

449,670

449,685

$+49,690$

449,709

$+49,710$

$4+9,719$

449,721

$+49,7 \geq 6$

$449,-31$

419,752

449,753

419,759

$4+9,766$

449,76 ;

449,774
Johnson, E. H

Martindale, W. B. and Malmborg, E. R

Schlesinger, W. M

Carty, J. J

Desant, W. F. Z

Lieb, C. A

Foster, H. A

Norman, T. W

Robbins. H. G

Schatz, A. E

Tiebb, J. IV

Blackwell, F. O

Blackwell, F. O

Crane, $\mathrm{M}$. G

Curtiss, G. F

Nitchell, A. W

Morse, S. F. B

ard, J. H. R

Ward, J. H. R

WTard, J. H. R

Fisher, B. $\mathrm{H}$

Nelson, R. E

Humans, II

Robinson, A

Lean, G. R

Lahmerer,

Naxstade, F. W

Strecker, II. P

Tan Nuis, C. S

Tarlev, R.

Andrus, D. P

Depp, O.J

Thomson, $\mathrm{F}$

Thomsou, $\mathrm{F}$

Tressel, F.

Dean, J. H

Mever, $J$. and Binder,

Bisliop, J. D

Cooley, F

Dewer, II. II

Easton, J. II

Dawnah, G

Gibbs, G

Mitcliell, A. IT

Cole, F. IT

Cole, F. II

Cole, F. IV

Ioflitt, $\mathrm{R}$. $\mathrm{F}$

Baxter, W., jr

Tompkins, $\mathrm{S}$

Bartsch, E.

Anderson, $\mathrm{F}$

Baxter, TV. j.

Baxter, W., ji

Finn, H. J. and Garner, J. A

Jacques, W. IT

Lron, J. B

Newman, $A$

Short, S. H

Thomson, E. and Rice, E. W., jr _..........

Ward, B. B.

Wheeler, G. K.

Ball, C. M. and Norton,

Carlton J A and Johnston,

Brown, H. P

Bromnlee, J. N

Martindale, W. B

Shubert, H. C

Tregurtha, $A$. $T$

Duenner, $\mathrm{H}$
Lighting, systems

Printing, hand stamps

Stone working, drills

Telephones, systems

Cash indicators

Towers, telegraph poles

Lights, arc, special features

Conductors

Telegraphy, transmitters

Telephones, details

Batteries, thermal

Cut-outs, mechanical

Railways, systems, conduit

Electric locomotives

Bells

Cut-outs, thermal

Railwars, systems, orerhead, trolleys...-.-.

Railways, systems, circuits, multiple arc

Lights, arc, special features

Lights, arc, gravity feed, gear-clutch

Regulators

Cash registers

Telephones, magnetic -

Heaters

Light, incandescent, special features

Cut-outs, mechanical

Regulators .

Cut-ruts, thermal

Batteries, galranic

Cut-outs, mechanical

Bells

Connectors

Medical and surgical, body-wear

Cint-outs, mechanical

Ileaters

Electro-magnets

Totors

Conductors

Lamps and gas fittings, reflcetors

Lamp shades

A larms

Scale beams

Lighting, srstems, car

Railways, srstems, orerhead, trollers-

- do - fire - -

Lights, sockets and keys _._._._._........

Separators - -

Applications, tools and machines

Electrodes

Telegraphy, automatic

Motor

Applications, tools and machines -........

Telegraphy, railway car

Telephones, systems

Telephones, switch-boards

Annunciators -...-...

Lights, are grarity feed, clutch-

Locomotire head lights

Connectors

Separators

Railway signals, block srstems

Electric locomotires.

Towers, telegraph poles

Printing, hand stamps

Galvanometers

Lights, incandescent, special features ......

Magneto-electric -........

Mar. 24, 1891

Mar. 2t, 1891

Mar. 24, 1891

Mar. 31, 1891

Mar. 31, 1891

Mar. 31, 1891

Mar. 31, 1891

Mar. 31, 1591

Mar. 31, 1891

Mar. 31, 1891

Mar. 31, 1891

Mar. 31, 1891

Mar. 31, 1891

Mar. 31, 1891

Mar. 21, 1591

Mar. 31, 1891

Mar. 31, 1891

Mar. 31, 1891

Mar. 31, 1891

Mar. 31, 1891

Nar. 31, 1891

Nar. 31, 1891

Mar. 31, 1891

Мат. 31, 1891

Mar. 31, 1891

Mat. 31, 1891

Mar. 31, 1891

Mar. 31, 1891

Mar. 31, 1891

Már. 31, 1891

Mar. 31, 1391

Mar. 31, 1591

Mar. 31, 1891

Mar. 31, 1891

Mar. 31, 1891

Mar. 31, 1891

Mar. 31, 1891

Mair. 31, 1891

Mar 31, 1891

Mar. 31, 1891

Mar. 31, 1891

Mar. 31, 1891

Mar. 31, 1891

Mar. 31, 1891

Mar. 31, 1891

Mar. 31, 1891

Mar. 31, 1891

Mar. 31, 1891

Mar. 31, 1891

Mar. 31, 1891

Mar. 31, 1891

Yar. 31, 1891

Mar. 31, 1891

Mar. 31, 1891

Mar. 31, 1891

Mar. 31, 1891

Mar. 31, 1891

Apr. T, 1891

Apr. 7, 1891

Apr. $\bar{T}, 1891$

Apr. 7, 1891

Apr. 7, 1891

Apr. 7, 1891

Apr. 7, 1891

Apr. 7,1891

Apr. T, 1891

Apr. 7, 1891

Apr. 7,1891

Apr. 7, 1891

Apr. 7, 1891

Apr. 7, 1891

Apr. 7, 1891

Apr. T, 1891 
No.

NAME.

DATE.

449,777 Hall, O. B

$+49,730$

$449-9.9$

$+49,-97$

449,629

449,532

449,836

$+49,543$

49,947

449,866

449,856

449,889

449,890

449,597

$+49,902$

449,903

449,921

49,923

449,943

449,977

449,990

450,000

450,068

450,074

450,099

450,101

450,103

450,104

450,105

450,153

450,159

450,163

450,164

$450,17$.

450,173

450,152

450,184

450,189

$45(1,193$

450, 206

450,219

450,221

450,228

4.5), 239

450, 242

$4.50,245$

4.5), 260

450,272

4.50, 28.5

450,292

450,294

$4.50,304$

450,319

450,354

450,358

450,359

$4.00,367$

450,384

450,390

450,425

$450,4 \div 6$

450,441

450,458

450,460

450,466

450,489

$4,50,541$

$4.50,542$

450,043

450,544

450,551

$450,5.52$

$450,5.58$

450,577

450,581

450,586
Yiüller, F, and Greene, J, G

geuni, E. 1

Thiomson, E

Blinchard, G. II

Eggers, F. F

Robinson, $\mathrm{F}$, and Lander, P. W. J

Turner, G. E

Zingsen, F. W

Brown, R. G

Easton, J. IV

Tregurtha, A.

McCarthr, I

stern, W. A

Wilhelm, C. II

Carpenter, H. H

Grefen, $\mathrm{H}$

Hnnter, R. M

Fahnehjelm, O. B

Gibbs, J.S

Le Sueur, E. A

e sueur, $E$. A

Le Sueur, E. A

Hickler, A.S

ones, A. IT

Lieb, C. A

Manstield, F

IIansfield, F

Nores, B. J. and Eaton, H. F

Pocock, F. A

Robinson, C. A

Wessel, F. A

Collins, W. F

Cunrnglame, H. H., Rawson, F. L. and IVoodhouse, O. E.

Fisk, P. H

Lieb, C. A

Ludlow, S. IY

Singer, IT. F

Wickes, R. $\mathrm{O}$

Crowdus, W. A

Johnston, Wr. C., jr--.-.

Lieb, C. A. and Learers, E

Van Choate, S. F

Pragnell, G. E

Burnley, W

Harcus, IT. $\mathrm{I}$

Eickemever, $\mathrm{P}$

Bertolette, D. A

Varley, R., jr

Reinemann,

Whipple, F. F

Gruschow, R. H

Hines, $D$

Ludlow, s. W

Jackson, W. E., jr

Van Depoele, C. J

I an Depoele, C.

Van Depoele, C. J

Atwood, Ia M. C

Cox, W

Davis, J. H

Gatley, E. J

Hnnter, P. M
Bentley, E. MI

Hunter, R. M

Slater, IV. IV

Eiston, J. II

Kirnan, TI.

Marcus, W. N

Van Depoele, C.

Adam, F. and Knapik, J
Towers, telegrapli poles

Lighting, systems

Railways, systems, overhead

Railways, systems, conduit

Telegraphy, fire, alarms and extinguishers

Bells

Heaters

Car brikes

Dental instruments

Bells

Railways, systems, overhead, trolleys _......

Electro-deposition

Telegraplıy, multiplex

Drop lights and hangers

Telegraphy, lightning arresters

Lights, incandescent, special features

Type-writing

Railways, conductor supports and insulators

Towers, telegraph poles

Railway signals, sectional rail

Lights, incandescent, special features

Magnetic locks and bolts

Railways, systems, overhead

Gas burners, incandescent

Cut-outs, thermal

Electrolysis:

Batteries, galvanic

Railways, systems, orerhead, trolleys

Cut-outs, thermal

Railways, conductor supports and insulators

Railways, systems, closed conduit do

Telegraphy, fire

Railways, systems, overhead, trolleys

Railways, grippers, whelp wheel

Ruilway's, systems

Conductors, underground

Magneto-electric

Galvanometers

Telegraphy, printing

Telegraphy, fire _......................

Railways, conductor supports and insulators

Telegraphy, fire, alarms and extinguishers

Telegraphy, circuit closers, thermal

Telegraphy, relays and sounders

Batteries, galvanic

Tclegraphy, circuit closers -............-

Railways, conductor supports and insulators

Lights, filaments and carbons

Bells .

Batteries, galvanic

Telephones, details

Telephones, supports

Magneto-electric

Insulators

Annunciator

Teleoraphy, circuit closers, thermal

Telephones, electric

Motors

Annunciators, drop

Railways, track cleaners, wheel fenders...

Alarms -

Railways, systems, oyerhead, trolleys

Transmission of power

Electric locomotives _-

Motors, reciprocating

Motors, alternating

Cut-outs, mechanical

Magneto-electric -

Annnnciators

Lights, arc, special features

Railways, systems, overhead
Valre-controller

Induction coils

Apr. T, 1891

Aur. T, 1891

Apr. 7,1891

Apr. 7,189

Ipr. T, 1891

Aur. 7, 1891

Apr. ఫ, 189

Apr. 7, 189

Apr. 7.1891

Aur. 7,1891

Aur. T, 1891

Apr. 7,189

Apr. 7, 159

Apr. 7,189

Apr. 7,1891

Anr. $\bar{T}, 189]$

Aur. 7, 1891

Apr. T, 1891

Apr: 7,189

Apr. 7,1891

Apr. '̄, 1891

Apr. 7,1891

Apr. 7,1891

A pr. T, 1891

Apr. 7, 1891

Apr. 7, 1891

Apr. T, 189

Apr. $\quad \bar{T}, 1891$

Apr. 7, 1891

Ipr. 14,1891

Apr. 14, 1891

Apr. 14, 1891

Apr. 14, 1891

Apr. 14, 1891

Apr. 14, 1891

Ajr. 14, 1891

Apr. 14, 1891

Apr. 14, 1891

Lur. 14, 1891

Apr. 14, 189

Apr. 14, 1891

Apr. 14, 1891

Apr. 1t, 1891

Apr. 14, 1891

A]1. 14, 1891

Apr. 14, 1891

Apr. 14, 1891

Apr. 14; 189

Apr. 14, 1891

A pr. 14, 189

Apr. 14, 1891

Apr. 14, 1891

Apr. 14, 1891

Apr. 14, 189

Apr. 14, 1891

A pr. 14, 1891

Apr. 14, 1891

Apr. 14, 1891

Apr. 14, 1891

Apr. 14, 1891

Apr. 14,1891

Apr. 14, 1891

Apr. 14, 1891 
No.

NAME.

450,557

450,58

450,60 .

450,613

450,61

450,620

$450,6 \div 8$

450,630

450,631

450,639

450,641

450,640

450,646

$450,65 \%$

450,683

450,68

450,690

450,706

45070

450,734

450,74

$450,7$.

450,834

450,839

$450,8+10$

450,810

450,853

450,87

450,075

450,596

450,923

450,927

50096

450,470

$+50,97$

450,97

$450,9 \mathrm{~s}$

$450,9.1$

401,037

451,10

451.10 .

451,121

451,19

$4 \overline{1} 11$ '

451,14

451,14

tร1.1.

451,155

$4.51,15$

451.17

451,159

$451,1.90$

$4 \overline{1 i} 1,200^{\circ}$

451,208

451,211

451,212

451,220

$4 \overline{1} 1,230$

$+51,240$

451,24

451,250

451,267

451,26

4.51 . 25

453,300

451,30

451,309

451,312

451,320

451.326

451,34

451,364

451,369

451.370

451.377

$451,3 \%$

451.397

Iarsh. J. II

culver, F.S

Delany, P. B

Kerstein,

Linville, S. R

Seiverling, J. F

stanley, W., jr

Taylor, J. D

Taylor, J. D

Westinghouse, G., jro

Short, S. II

Thomson, E

ITilson, T.S

Tinklıam, L. B

Creighton, $\mathbf{T}$

Bunker, W. I

Johnson, E. II

Jolinson, E. H

Currie, s. C. C

Willms, C

Steplicnson, J

Webbed; II.

Reardon, C. B

Sims, WT. S

ms, J. C

Woolley, L. G

Matthews, F.

llower, s. E

Norton, J. A

Parcelle, .1. I

Willi:uns, J. A

artwright, D.

Frost, L. J

loung, C.

taynor, T. F

lving, F.K

an Gestel, J. T

Bates, P. S

Hunter, R. II

flunter, R. II

Jackson, L.

Emmet, W. Le

IVard, J. H. F

IVhite, Lc R.

Thomson, D. P

Edwards, $W^{\top} . C$

Macrae, $\mathrm{R}$

Sharp, E. I -

Ricketts, J. I

Dewey, M. II

Dewer, M. IY

Grant, P

ITaterhous

Clark, H. T

Drew, H. P

Park, H. \&

Sclief bauer, $\mathrm{R}$

Eiekemeyer,

Jennings, $\mathrm{B}$

Thomson, $\mathrm{E}$

Bretrer, H. J

Conkling, J.P

Conkling, J. P

Haines, R. D

Chase, H. A
Lieb, C. A. and Lavens, E

Alton, G. IH

Linville, S. R. and Hettmansperger, L. F

Danals, C A and lerry, II. O

Villiers-stead, F. and Ifedgman, E. R

St. John, S. H. and Jennings, F. A

Tischendoerfer,
Class.

IDATE.

Railways, conductor supports and insulators Conductors

Lights, sockets and keys

Railways, systems, overhead

Annunciators

Lighting, systems

Lights, sockets and key

Telegraphy, printing

Iragneto-electric

Regulators

Telephones, calls

Railway signals

Railways, systems, orerhead, trolleys

Railways, systems, overhead

Electrodes

Telegraphy, circuit closers

nstilators.

Conductors

Electric locomotive

Gearing

Batteries, secoudary

Conductors, lead-covered

Batleries, galvanic

Electric locomotives

Railways, systems, overhead, trolleys

Applications, gencral

Steering and propulsion

Telegraphy, circuit closers, thermal

Induction coils

Valve controllers

Watelı stops

Electric locomotives

Cut-outs, meclianical

IIagneto-electric

R'egulators

Lights, incandescent, special features

Carburetors, gas and air mixcrs -

Liglits, arc, gravity feed, gear-cluteh

Digliop arce gravity feed

Bells

Meters

Batteries, galranic

Telephones, srstems_.......................

Lights, incandescent, special features - .

Railwars, systens, circuits, multiple arc

Electric locomotires

Railway signals.

IIagneto-electric

Lights, are, gravity feed, gear-cluteh

Drop lights and hangers._._._._.....

Separators

Railways, swstems, orcrhead

Ulotors

Annunciators, diop

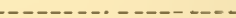

Iagneto-electric

Lights, arc, grarity feed, clutch

Cut-outs, mechanical do

Station indicators

Lights, incandescent specinl features - -

Lights, arc grarity feed, gear-clutch

Regulators

Motors, reciprocating

Railwars, srstems, conduit -..........

Heateris

Connectors

Separator

Lighting, systems

Insulators

Telephones, switch-boards

Apr. 14,1891

Apr. 14. 1891

Apr. 21, 1891

Apr, 21, 1891

Apr. 21, 1891

Apr. 21, 1891

Apr. 21, 1891

Apr. 21, 1891

Apr. 21, 1891

Apr. 21, 1891

Apr. 21, 1891

Apr. 21, 1891

Apr. 21, 1801

Apr. 21, 1891

Apr. 21, 1891

tpr. 21, 1891

Apr. 21, 1891

Apr. 2], 1891

Apr. 21, 1891

Apr. 21, 1891

Apr. 21, 1891

Apr. 21, 1891

Apr. 21, 1891

Apr. 21; 1891

Apr. 21, 1891

Apr. 21, 1891

Apr. 21, 1891

Apr. 21, 1891

Apr. 21, 1891

Apr. 21, 1891

A pr. 21, 1891

Apr. 21, 1891

Apr. 21, 1891

Apr. 21, 1891

A pr. 21, 1891

Apr. 21, 1891

Apr. 21, 1891

Apr. 21, 1891

Apr. 28, 1891

Apr. 28, 1891

Apr: 28, 1891

Apr. 28, 1891

A pr. 28, 1891

A pr, 28, 1891

Apr. 28, 1891

A pr. 28, 1891

A pr. 28, 1591

Apr. 28, 1891

A pr. 2S, 1891

A pr. 28, 1891

A pr. 28, 1891

Apr. 28, 1891

Apr. 28, 1891

Apr. 2S, 1891

Apr. 28, 1891

Apr. 2S, 1891

Apr. 28, 1891

Apr. 28, 1891

Apr. 28, 1891

Apr. "28, 1891

Apr. 28, 1891 
Yo.

Naye.

451,402

$4.51,407$

451,41

451,445

451,449

\$51,491

451,512

451,514

$451, \overline{9} 20$

451,521

451,540

451,541

451,548

451,559

$451,5 i t$

451,596

451,557

451,603

451,605

451,611

451,614

451,634

451,637

451,639

451,649

451,656

451,666

451,689

451,691

451,692

451,696

451,700

451,730

451,732

451,786

451,798

451,815

451,832

451,870

451,872

451,873

451,584

451,885

451,894

451,902

$+51,909$

451,921

451,922

451,933

451,941

451.942

451,94

451,950

451,951

451,960

451,980

451,981

451,996

451,997

451,999

452,008

$452,(4) 5$

452,017

452,026

452,030

$452,03$.

152,036

452,041

452,042

452,056

452,068

452,012

$4:, 2,091$

$4 \overline{2} 2,099$

452,151

452,152

Hunter, R. II

Lloyd, H. H

Storer, 1

Aldrich, T. H

Booth, A. C

Kniglit, W. $\mathrm{H}$

Noyes, B. J

Payne, J. N

Rice, E. W', jr

Washburn, G.

Washburn, G. A

Zagelmerer, I

H Yer, W. E

Rice, L. C.

Tilliams, $\mathrm{J}: \mathrm{B}$

IVilliams, J. B

Sterry, T. N

Andrews, J. D. F

Allison, M. H

Corwin, H.S

Harding, C. $\mathrm{K}$

O'Donnell, M. A

Dayton, C. A

Gee, E. C

Davis, M. G

Holland, J

Hunter, R. I

Kuehnle, J

Poland, L. X. P

Fletcher, J. P

Jones, B. J

Van Depoele, C. J

Forl, W. H

Milliken, F

Hewett, E. M. G

La Boiteaux, F. M

La Boiteaux, F. M

Wheeler, S. S

Wheeler, S. S

Collins, WV. F

Stover, J. W

Blades, H. H

Emmuer, J., jr

Farnham, I. H

Hunt, A. II son, C. $\mathrm{A}$.

Lechner, F. MI

McCnllough, G. C

Oakman,

Pope, E -

Tracy, L. W

Short, S. H

Short, S. H

Dow, WT. E

Dow, W. E

Lipe, C. E.

Short, S. H

Lieb, C. A

Kintner, C. J

Castner, H. Y

short, S. H

Short, S. I

Pies, E. E

Pies, E. E:

Ashrell, W. H

Doncet, S. J

Williams, J. B

Dewey, M. II

Whitlingham, G. H

Whittingham, G. H
Deacon, S. and Wightman, M. J

Balsley, C. $\mathrm{H}$ and Porter, $\mathrm{E}$. II

La Point, T. T., Flanagan, J. H. and Thomp-
Cluss.

DATE.

Railways, systems, overliead, trolleys

Bells

Batteries, secondary

Telegraphy, repeaters

Electric locomotives

Telegraply, fire

Car brake

Meters

Telegraphy, lightniug arresters

Batteries, secondary

Telegraphy, circuit closers, therma

Motors

Magneto-electric

Cnnductors

Applications, general

Conductors

Fare registers

Conductors, underground

Telephones, magnetic

Railways, systems, closed conduit

Telegraphy, fire

Valve controllers

Lights, sockets and keys

Time checks

Telegraply; fire -..

Railways, systems, closed conduit

Railways, systems, conduit

Raiiways, systems, overhead

Magneto-electric

Towers, telegraph poles

Lighting systems, car

Transmission of power

Lights, sockets and keys

Towers, telegraph pole

Motor:

Pneumatic fan

Magneto-electric

iscellaneous

Iagneto-electric

Telegraphy, fire

Lagneto-electric

Battcries, secondary

Telephones, toll

Connectors

Drain tiles

Mining machines

Batteries, galvanic

Insulator

Telegraphy, printing

Lead pipe making

Electric locomotives

Drop lights and hanger

Cut-outs, thermal

Railway signals, cab, sectional rail .......

Electro-ma

Electric locomotives

Railways, conductor supports and insulators

Telegrapliy, transmitters

Reducing and separating

Electric locomotives

Car brakes

Heaters

do

Coin controlled apparati

Railway signals, cab, sectional rail

Conductors

Railways, systems, double

Motors

Cut-outs, thermal

Apr. 28, 1891

Apr. 25, 1891

Apr. 2s, 1891

May 5, 1891

May 5, 1891

May 5, 1891

May 5, 1891

May 5,1891

May 5, 1891

May 5, 1891

May 5, 1891

May 5, 1891

May 5, 1891

May 5, 1891

May 5, 1891

May 5, 1891

May 5, 1891

Mar 5, 1891

May 5, 1891

May 5, 1891

Iay 5, 1891

May 5, 1891

May 5, 1891

May 5, 1891

May 5, 1891

May 5, 1891

May 5, 1891

May 5, 1891

May 5,1891

May 5, 1891

May 5, 1891

May 5, 1891

May 5, 1891

May $5,1.891$

May 5, 1891

May 5, 1891

May 5̄, 1891

May 5, 1891

IIay 5, 180

May 5, 1891

May 5, 1891

May 5, 1891

May 5, 1891

May 5, 1891

May 5, 1891

May 12, 1891

May 12, 1891

May 12, 1891

May 12, 1891

May 12, 1891

May 12, 1891

May 12, 1891

May 12, 1891

Iay 12,1891

May 12, 1891

May 12, 1891

May 12,1891

Mas 12, 1891

Lay 12, 1891

May 12, 1891

May 12, 1891

May 12, 1891

Way 12, 1891

May 12, 1891

May 12, 1891 
No. NAME.

452,160 452,165

452,160

452,173

452,176

452,180

$+52,201$

452,214

$+52,220$

152,250

$452,25,5$

452,276

452,281

(1)

452,290

452,299

452,303

452,305

452,306

452,326

452,340

452,341

452,342

452,359

452,367

$452,39=$

452,397

452,420

452,402

452,423

$452,42$.

452,420

452,494

52.50

452,510

$452,51$.

450

$+5,2,516$

$45^{2}, 72$

452,541

$452,036]$

$+52,565$

452,571

452,74

452,570

452,58 .

452,599

452,611

452,619

452,621

452,622

452,633

45060

452,63

$452,64.7$

452,701

452,717

452,718

452.2

452,73

$452,7+1$

452.758

452,760

$452,76$.

452,57

452,775

452,780

452,782

452,795

452,796

452,799

$452,8 \div 1$

45,872

452,878

45285

452,855

Blackivell, F. O

crane, M. G

Andrews; J. D.

Jones, R. M

Thateher, E. H

Chisholm, D. J

Gunning, J. H

Wílliams, C. D

Bray, T. J

Miller, H. IV

O'Neil, J. C

Savage, IV. IV

Van Depoele, C.J

Wiseman, A. G

Hanson, IV

Keen, IV. M. B

Westnn, E

Conner, W. A

Conner, W. A

Conner, W. A

Briggs, $\mathrm{C} . \mathrm{T}$

Fischer, J. G

schmidt, F. M

Straus, M

WTessel, l: A

Black well, F. O

blackwell, F.

Church, 1. II

Gray, E

Fessenden, R. A

Iilumpp, J. F., jr

Willians, J. B-

Beck, $\mathrm{O}$

Brown, F, H

Jennings, B

Cook, D. N.

Bartholonew, 1. H

Curry, IV

Holson, A. B

Jenner, C, D

Kuelnie, J

Dooley, T', B

Mitchell, E. II

Shaw, A. J

Short, S. H

Short, S. I -

Buchinan, F

Fitzimmons, $\mathrm{P}$

Jennings, $B$

Riker, A. I

Riker, A. I

Williams, J. B

Shatfer, C. H

Weems, D. G

Míller, A. C. F

Salatlé, F

Chase, H.

Dickson, C. T

Flagg, I. J

Hamrick, H. I

Kintner, C. J

Matteson, J.

Deming, $\mathbf{E}$

Deming, $\mathbf{E}$

Deming, E

Deming, E

Faror, L. B rane, M. G. and Cole, F. WY

Keen, W. M. B. and Haarmann, W. F

Carroll, E. and Lambert, T. B

Gärtwer, A. F. and Harris, J.

Gärtner, A. F and Harris, J.
Cluass.

DATE.

Railways, systems, conduit

Annunciaturs

Cut-outs, mechanical

Electric locomotives

Railways, systems, overhead, trolleys

Railways, elevated

Motors

Electrodes . . ..........................

Medical and surgical, body-rear.

Towers, telegraph poles

Meters -...-.

Railway signals

Lights, incandescent, special features.

Transmission of power-

Clocks, electric

Batteries, galvanic

Jights, sockets and keys

$$
\text { do }
$$

Galvanometers

Conductors, lead-covered do

Motors

Lights, incundescent, special features

Clocks, electric .....................

Telegraphy, circuit closers

Magneto-electric --

Clocks, electric

Motors

Lights, incandescent

Telegraphy, fire, alarms and extinguishers

Conductors

Advertising, signs

Railway signals, cab, sectional rail

Railways, conductor suuports and insulators

Railways, systems, overhead, trolleys

Magneto-electric

-

Applications, general

Railyars, systems, overhead

Annmnciators, drop

Viscellaneous compositions

Railways, systems, closed conduit

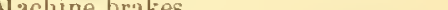

Electric locourotives

- - - do - -

Telephones, electric

Lamps and gas fittings, globes

Railways, conductor supports and insulators.

Lamps and gas fittings, globes

Iagneto-electric.

- - - do

Telegraphy, fire, automatic-

Railways, systems, telpher

Telephones, electric

Insulating materials

Telegraphy, fire

Telephones, details

Lichts, incandescent

Telephones, systems, toll ...........................

IIotor

Lightning rods .

Drop lights, spring drum

Telegraphy, railway-car

Railway signals, cab, sectional rail

do

Cut-outs, thermal

May 12, 1891

May 12,1891

May 12, 1891

May 1?, 1891

May 12, 1891

May 12,1891

May 12, 1891

Mav 12,1891

May 12, 1891

May 12,1891

May 12, 1891

May 12, 1891

May 12, 1891

May 12,1891

May 12,1891

May 12, 1891

May 12, 1891

May 12, 1891

May 12, 1891

May 12, 1891

May 12, 1891

Lay 12, 1891

May 12, 1891

May 19, 1891

Nay 19, 1891

May 19, 1891

May 19, 18:1

May 19, 1891

May 19, 1891

Nay 19, 1891

May 19,1891

May 19, 1891

May 19,1891

May 19, 1891

May 19, 1891

May 19, 1891

May 19, 1891

May 19, 1891

Hay 19,1891

May 19, 1891

May 19, 1891

May 19, 1891

May 19, 1891

May 19, 1891

Iay 19,1891

May 19, 1891

May 19, 1891

May 19, 1891

May 19, 1891

May 19, 1891

May 19, 1891

May 19, 1891

May 19,1891

May 19, 1891

May 19, 1891

May 19, 1891

May 19, 1891

May 19, 1891

May 19, 1891

May 19, 1891

May 19, 1891

May 19, 1891

May 19, 1891

May 19,1891

Мay 19, 1891

May 26, 1891

May 26, 1891

May 26, 1891 
452,89t Bundy, W. Le G

$45 ?, 913$

$45: 920$

452921

$45,9,931$

452,932

$45: 2,934$

452,935

452,936

452,937

452,941

452,942

4.5.,951

$452,95 \overline{5}$

452,956

452,983

452,992

453,000

453,002

453,029

453,031

453,032

453,036

$453,0+1$

$\pm 53,046$

$+53,0 \div 0$

453,093

453,097

$+53,100$

t58,104

453,111

$+53,162$

453,163

453,164

$4.53,167$

$+53,190$

453,216

+5 3,225

453,230

453,242

453,248

453,260

453,261

453,281

$4 \overline{3} 3,286$

453,28

453,317

453,318

4. 3,319

4.53,320

453,323

453,326

453,337

453,349

$453,35 \overline{5}$

+5) 3,367

453,416

$4.53,430$

4. 3,435

$45 \overline{3}, 447$

$4 \overline{3} 3,45 \overline{5}$

453,466

$+53,476$

453,483

453,484

$453,5,-2$

$+5 \overline{3}, 578$

453,581

453,583

$4 \overline{3} 3,597$

$4.53,601$

453,618

453,681

453,693

453,695

453,696

Edison, T. A.

Hunter, R. M-

Lyon, J. B

Cole, F. IV

Freeman, W. K

Freeman, W. K

Freeman, IV. K

Scribner, C. E

Slater, W. W

Thomson, E

Prentiss, H. S

Prentiss, H. S

Dobbie, R. S

Goodridge, W. M

Hamilton, J. E

Warner, E. P

Baker, E

Blades, H. H

Brady, T. H

Drysdale, W. A

Hall, G. I

Wheeler, G. K

Sullivan, J

Branch, J. $\mathrm{B}$

Edson, W

Ries, E. E

Ries, E. E

Ries, E. E...

Jackson, S. D. I

Shawhan, J. F

Wheeler, F

Bundy, W. I

Humans, W

mith, T. C

Kells, C. E., jr

Langdon-Davies,

Young, C. G

Lieb, $\%$ A

Macrae, $\mathrm{R}_{\text {- }}$

Townsend, H. C

Wessel, F.A

Wessel, F. A

Wessel, F. A

Ebert, W. H

Ide, A. L

Davis, C. E

Sawyer, R. F

Haskins, C. C

Pinolet, L. M

IcLanghlin, J. F

Lnby, W. A

White, Le R. S

Darling, E. A.

Harness, C. P

Jacques, W. W

Jewell, R.

Baumann, $\mathrm{R}$

Chapman, N.

Fitzimons, $\mathrm{P}$

Hochhausen, W

La Roche, F. A

Edison, ' $\Gamma$. A

Brown, H. B.

Wagner, F. C

Ells, J. W

Griscom, IV. W

Hadlock, W. E:
Walter, W. C-

Baker, W. E. and Macloskie, C. H.

Haswell, A. E. and A. G

Tinning, F. T. and W. K.S

Furlong, L. F. and Tucker, F. O
Time checks

Telegrapliy, duplex

Railways, systems, conduit _............. May 26, 1891

Telephones, switch-boards _............. May 26, 1891

Bells

do - lectric

Magneto-

Motors

Dynamometers

Railway brakes, electri

Magneto-electric

Clocks, electric

$$
\text { do }
$$

Lightning rods

Electric locomotives

Telephones, switch-boards

Harps and hangers

Cut-nuts, thermal

Railways, systems, telpher

Motors

lnsulators

Lights, incandescent, special features

Lighting, systems -

Railways, conductor supports and insulators

Railways, systems, overhead, trolleys

Railway signals, cab, sectional rail

Railways, conductor supports and insulators

Railway signals, cab, sectional rail

Cut-outs, thermal

Tempering and annealing

$$
\text { do }
$$

Heaters

Electric locomotives

Advertising, alıtomatic devices

Electric locomotives

Connectors

Time checks

Magneto-electric, hand machines

Lighting, systems -

Dental instruments

Telegraphy, reed

Lighting, systems

Towers, telegraph poles

Motors

Separators

Regulator

do

Lighting, systems, test.

Railways, conductor supports and insulators

Cut-outs, thermal

Lighting, systems

Electro-deposition

Motors

Annunciators

Type-writing

Telephones, supports .-

Railway signals, sectional rail

Lights, sockets and keys _...-

Alarms, burglar

Electrodes

Telephones, systems

Measuring pumps

Cut-outs, electro-magnetic

Railway signals, cab, sectional rail

Telephones, electric

Lights, are, gravity feed, clutch

Lighting, systems....

Telegraphy, multiplex.

Igniting devices, manual

Cut-outs, mechanical

Galvanometers

Lights, arc, positive feed, gear-clutch

Batteries, secondary

Indicators

May 26, 1891

May 26,1891

May 26, 1891

Nay 26, 1891

Jlay 26, 1891

May 26, 1891

May 26, 1891

May 26,1891

May 26, 1891

May 26, 1891

May 26, 1891

May 26, 1891

May 26, 1891

May 26, 1891

IIay 26,1891

May 26, 1891

May 26, 1891

May 26, 1891

May 26, 1891

May 26, 1891

May 26, 1891

May 26, 1891

May 26, 1891

May 26, 1891

May 26, 1891

May 26, 1891

May 26, 1891

May 26, 1891

May 26,1891

May 26,1891

May 26, 1891

May 26, 1891

June 2, 1891

June 2, 1891

June 2,1891

June 2, 1891

June ?, 1891

June 2, 1891

June 2, 1891

June 2,1891

June 2, 1891

June 2, 1891

June 2, 1891

June 2, 1891

June 2, 1891

June 2, 1891

June 2,1891

June 2, 1891

June 2, 1891

June 2, 1891

June 2, 1891

June 2, 1891

June 2,1891

June 2,1891

June 2, 1891

June 2, 1891

June 2, 1891

June 2, 1891

June 2, 1891 
No.

453,710

453,722

453,723

453,733

453,742

453,822

453,858

453,861

453,868

453,871

453,875

453,88

$453,8.98$

453,950

453,98 :

453,998

453,995

454,008

454.016

454,018

454,020

454,02

454,02 ?

$454,02:$

454,02

454,031

454,038

454,057

454,060

$4.54,087$

454,090

454,091

454,09 .

454,138

454,150

$45+166$

454,17

454,17

454,181

454,18

454,157

454.07

454,220

454,23

$45+245$

454,25

$454,200^{\circ}$

454,270

454,275

454,294

$454,29=$

454,313

454,336

454,338

454,344

454,404

454,430

454,437

454,443

454,450

454,462

454,475

454,476

454,450

454,486

454,487

454,488

$45+, 496$

454,503

454,504

454,522

454,529

$454,53 \div$

$45+, 536$

454,541
Name.

Class.

Date.

Jones, J.

Baker, F. E

Braun, M. N

Price, J. E

Sperry, E. A.

Oavis, YI. and Stokes, A. H

Halpine, N.J.

Hibbard, A. S

Stovell, O. H

Atterbury, T. B

Mitchell, IV

Brinkerhoff, J. O. and Smith, M. E

Valter, J. H

Milliken, G. F

Cole, F. WY

Currie, S. C. C

O'Connell, J. J.-.

Bacorn, F. W

Bentley, E. M

Bentley, E. I

Bentley, E. MI

Blades, H. H

Clark, H. T

Fanning, J. .

Macmillan, IV

MaCracken, E. D

Teague, F

Thomson, E

Tommasi, D. an

Mercadier, E. J. $\vec{P}$

Readman,

Dillon, T

Miller, J. J

Miller, J. J

Nash, E. L., Stout, G. G. and Daris, J. R.

Pattison, R. and Desmond, D. G

Sellon, J.

Eickeniever, R., jr

Pieper, O. H. and Watson, F. II

Ziegler, J. O

Clarke, $\mathbf{P}$

Edisun, T. A

II errick, A. B

Jolinson, E. If

Rushmore, S. IV

Rushmore, S. W

IT cub, G. F--

Essick, s. V. B

Guiley, A. H. R

Ames, $\mathrm{F}$

Greenfield, L. T

Hunter, R. M

Mc.Auley, J. E

Bassett, $N_{-}-$

ickemerer, $\mathrm{B}$

Hering, $\mathrm{C}$

Hering, C

Lemp, H. and Wightman, M. J

Lieb, C. A

Lieb, C. A

Lieb, C. A

Rae, F. B

Sundeen, A. D., Molander, S. B., Anderson,

G. II. and Carlsen, A. M.

sundeen, A. D., Molander, S. B., Anderson, G. W. and Carlsen, A. M.

Larkin, G. H. and Tomkins, J -

Rutter, G. E.

Verstraete, $\mathrm{E}$

Duncan, IT

Winkley, E. E-
Railways, systems, overhead-

Telephones, details

June 9, 1891

Telegraphy, circuit closers, thermal ....... June 9, 1891

Conductors, underground _._._._._._. June 9, 1891

Lights, incandescent _..._._._._._._._._. June 9, 1891

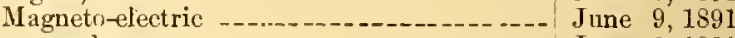

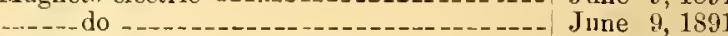

Ships, submarine and torpedo _..._......... June 9, 1891

Telephones, switch-boards ................ June 9,1891

Lighting, systems_-___._-_._-_._._._. June 9, 1891

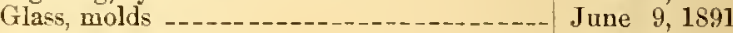

Cut-outs, mechanical _..._................. June 9, 1891

Batteries, galvanic ...____._._._._._._. June 9, 1891

Batteries, secondary-_-_-_-_-_-_-_-_-_-_-_ June 9, 1891

Telegraphy, fire _...................... June 9, 1891

Telegraphy, firc, non-interfering_..._._._.. June 9, 1891

Batteries, secondary ....................... June 9, 1891

Electric loemotives _...................... June 9, 1891

Telephones, srstems..._-_._._._._._._. June 9, 1891

Annunciators_....................... June 16, 1891

Electric locomotives _-__._-_._._._._._._. June 16, 1891

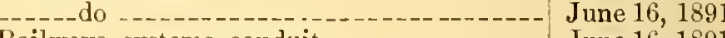

Railways, systems, conduit --_-_-_._._.-. June 16, 1891

Motors -

Cut-out, mechanical, oscillating snap _.... June 16, 1891

Ligliting, systems _._. . ...._._._._._._._. June 16, 1891

Batteries, galvanic _.._-_._._._........... June 16, 1891

Conductors__._._._._._._._._._._._._._. June 16, 1891

Cut-outs, mechanical _................... June 16, 1891

Inductoriums, transformers, stationary _- ._. June 16, 1891

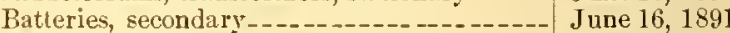

A pplications, tools and machines ..........-. June 16, 1891

Telephones, supports_..._._._._._._._... June 16, 1891

Conductors, underground _.................. June 16, 1891

Street lamp hangers _._._._-_._._..._...... June 16, 1891

Railways, systems, conduit -..-_-_-_._-_. June 16, 1891

June 16, 1891

June 16, 1891

June 16, 1891

June 16, 1891

June 16, 1891

June 16, 1891

June 16, 1891

J une 16, 1891

June 16, 1891

June 16, 1891

June 16, 1891

June 16, 1891

June 16, 1891

June 16, 1891

June 16, 1891

June 16, 1891

June 16, 1891

J une 16, 1891

June 16, 1891

June 16, 1891

Juue 16, 1891

June 16, 1891

June 23, 1891

June 23, 1891

June 23, 1891

June 23, 1891

June 23, 1891

June 23, 1891

June 23, 1891

Jnne 23, 1891

June 23, 1891

June 23, 1891

Telegraply, lightning arresters

Telephones, systems.---

Telephones, calls

June 23, 1891

Railways, systems, overhead, trolleys

Coin controlled apparatus, weighing

Railwavs, systems, overhead, trolleys.

June 23, 1891

Jnne 23, 1891

June 23, 189

June 23, 1891

Talre-controllers

June 23, 1891 


\begin{tabular}{|c|c|}
\hline So. & NaJie. \\
\hline $45 \pm, 5 \pm 6$ & Sperry, A. IV \\
\hline $45 \pm, 517$ & Sperry, A. IV \\
\hline $451,5 \pm 8$ & Sperry, A. W \\
\hline $454,5.5 .5$ & Atkins, C. H. \\
\hline $45 \pm, 5.58$ & Edison, T. A.. \\
\hline $45 \pm, 5,0$ & Moore, G. $\mathrm{K}$ \\
\hline 454,573 & Sherman, W. $\mathbf{N}$ \\
\hline 451,584 & Tiles, F. T. and Young, N \\
\hline 454,598 & Hathaway, P - \\
\hline $\begin{array}{l}45 \pm, 604 \\
45+, 622\end{array}$ & $\begin{array}{l}\text { Sautter, G- } \\
\text { Tesla, N }\end{array}$ \\
\hline $45 \pm, 625$ & Lenahan, F. P \\
\hline
\end{tabular}

Roney, A. B Toye, B. B

Eoote, G. L. and Moore, W. C

Herzberg, I. and $A$

Strauss, J. B

Thomson, E

Thomson, E

Angell, E. E

Decker, $\mathrm{P}$

Emmner, J., jr

Leonard, B. IV

Thomson, E

Bickford, J. H

Smith, G. IT

Ward, J. H. R

Anderson, $\mathrm{B}$

Bennett, E. S. and Parshall, H. F

Haight, H. J

Lawrence, TT. H

Scott, G. J

Scott, G. J

Sedgwick, F

Stephenson,

Thomson, E

Fletcher, J. R

Fletcher, J. R

Wood, F. B

Clansen, W. $\mathrm{H}$

Chenoweth, A. C

Chenoweth, A. C

Pilkington, H. M and Thite R. SSigsbee, C. D., Hayward, T. S. and Anderson, F.S

Martin, iI

Martin, $\mathrm{M}$

Drew, C. W. and Francis, E. R

Parker, E. H

Des Brisay, J

Palmer, D. A

Applezard, A. E

Harte, W. F. and Gerold, C. A

Gardner, F

Von Hefner-diteneck, F

Ramsay, W

Tesla, $\mathrm{N}$

Tesla, $\mathrm{N}$

Thompson, F. H

Van Hoevenbergh, $\mathrm{H}$

Wright, G. WV

Wright, G. W

Billberg, C. O. C. and Winand, P. A. N

Harris, A

Munsie, J. F

McGahan, F. L

IcGahan, F. L

Smith, W. A. M. and R. T. F.

Wass, D. D

Bution, $G$. and Wyeth, W. E

Erlwein, $G$...
Class.

DATE.

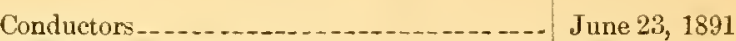

Insulating materials _........................ June 23, 1891$$
\text { do }
$$

Separators

Lights, incandescent

Figure wheeled toys

.....- June 23, 1891

Batteries, galvaníc _... June 23, 1891

Lights, are, special features

Lighting, systems _..._._._._._._._._. June 23, 1891

hailway signals, eab, sectional rail

Motor - June 23, 1891

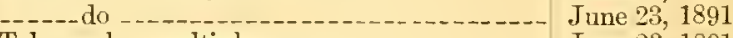

Telegraphy, multiplex_._._._._._._._. June 23, 1891

Telephones, calls, step-by-step _._-_._-_._-_- June 23, 1891

Lighting, systems _........ June 23, 1891

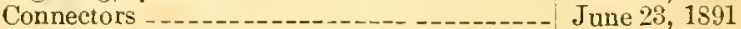

Telegraphy, lightning arresters_._._....... June 23, 1891

June 23, 1891

June 23, 1891

Heaters _........................................ June 23, 1891

Applications, general_.................... June 23, 1891

Batteries, galvanic ... June 23, 1891

Telegraphy, fire, automatic_._._._._._. June 23, 1891

Cut-outs, electro-magnetic_._._._._._. June 23, 1891

Counectors _._- June 23, 1891

Lights, arc, special features ..._....... June 23, 1891.

Telegraphy, circuit-closers _.............. June 23, 1891

Batteries, secondary _._._._._._._._._. June 23, 1891

Applications, tools and machines........ June 23, 1891

Indicators _............................... June 30, 1891

Lights, filaments and carbons _............ June 30, 1891

Railways, systems, overhead _...... June 30, 1891

Motors June 30, 1891

Inductoriums, transformers, rotary _...... June 30,1891

Telegraphy, printing ...................... June 30, 1891

Electric locomotives

Conductors, anti-inductive ............ June 30, 1891

Time locks _................................... June 30, 1891

Railways, systems, overhead _........... June 30,1891

Cut-outs, thermal ...................... June 30, 1891

Annunciators ............................. June 30, 1891

Cut-outs, electro-magnetic_._._._._._._. June 30, 1891

Conductors, underground ................... June 30, 1891

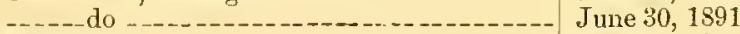

Meters _-_._._._._._._._._._._._._. June 30, 1891

Rheostats - ....... June 30, 1891

Telegraphy, fire, alarms and extinguishers-- June 30, 1891

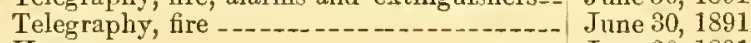

Heaters -.................................... June 30, 1891

Dampers, automatic -........ June 30, 1891

Cut-outs, mechanical ...................... June 30, 1891

Alarms, burglar _.._-_._-_._-_._._. June 30, 1891

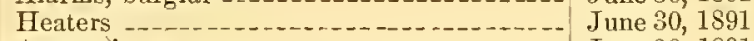

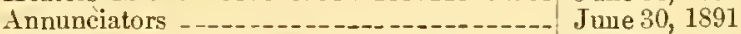

Railways, systems, battery _.................. June 30, 1891

Drilling and boring -.................... June 30, 1891

Clocks, electric _... June 30, 1891

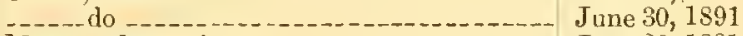

Motors, alternating - June 30, 1891

Meters _..... June 30, 1891

Lights, incandescent ............... June 30, 1891

Lights, are, gravity feed, clutch........ June 30, 1891

Telegraphy, printing, wheel _.............. June 30, 1891

Telegraphy, circuit-closers _............... June 30, 1891

Lights, arc, positive feed, gear-clutch .... June 30, 1891

Cnt-outs, electro-magnetic - June 30, 1891

Coin controlled apparatus, exhibitors, surface_. June 30, 1891

Railways, conductor supports, and insulators June 30, 1891

Motors June 30, 1891

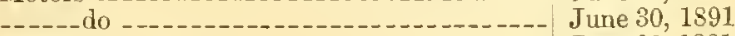

Post-office boxes . . June 30, 1891

Annunciators.............................. June 30, 1891

Amalganators, electric _..._............ June 30, 1891

Lights, filaments and carbons _......... June 30, 1591 
32

NUMERICAL INDEX-ELECTRICITY.

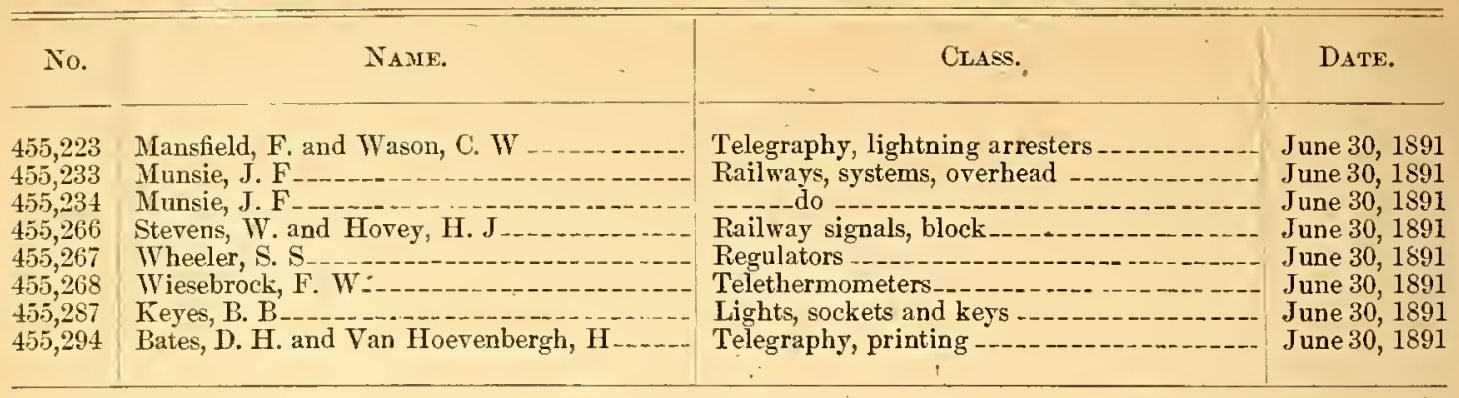




\section{APPENDIX No. X.}

PART III.

CONSISTING OF A

\section{ALPHABETICAL INDEX OF ALI PATENTS}

RELATING TO

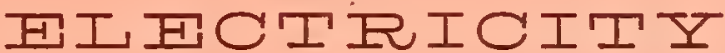

Issued from July 1, 1890. to June 30, 1891, inclusive, giving names of Inventors, numbers and dates of Patents, and the sub-class to which each belongs. 



\section{ALPHABETICAL INDEX}

OF

\section{Patents Relating to Electricity}

From July 1, 1890 , to June 3O, 1891.

\begin{tabular}{|c|c|c|c|}
\hline No. & AME. & CLAss. & DATE. \\
\hline $66 \pm$ & Abshage & Heaters. & Sept. 23.1890 \\
\hline & & -----do & Dee. 16,1890 \\
\hline & Abshagen, & do -. & Dec. 16,1890 \\
\hline & kermann, W. & Alarms, burglar & Oet. 7,1890 \\
\hline & Adams, A. IT & Railways, systems, overhead, trolleys & Dee. 2,1890 \\
\hline & Adam, F. and $\mathrm{F}$ & Cut-outs, mechanical & Apr. 14. 1891 \\
\hline & Adams, G. W & Telegraphy, fire, alarms aud extinguishers & Mar. 10,1591 \\
\hline & Adams, G. TT. & Time locks & June 30,1891 \\
\hline & D. A - & Railways, systems, & Sept. 16,1890 \\
\hline & A & Lights, incandescent & Tuly 29,1890 \\
\hline 592 & Ald & Cui-onts, therinal & Jan. 20,1891 \\
\hline & & Batteries, secondary _...... & May 5, 1891 \\
\hline 1 & Alliso & Fare registers ......... & May 5,1891 \\
\hline & Alton, G. I & Cut-outs, thermal & NoF. 18,1890 \\
\hline & Al & , sockets and keys --- & Apr. 21, 1891 \\
\hline 04 & An & Sewing machines, special machines & June 16,1891 \\
\hline 918 & Ar & Rheostats _........ & Dec. 2,1890 \\
\hline & & machines & Mar. $1 \overrightarrow{7}, 1891$ \\
\hline & tone, J. O & -electric .. & Nov. 18, 1890 \\
\hline 34 & Anders & Railways, systems, overhead, trolleys _-_- & July \&, 1890 \\
\hline 19 & An & -.-_- do & Oct. 11,1890 \\
\hline 18 & Ar & Batteries, secondary - & June 23,1891 \\
\hline 3 & An & Telegraphy, automatic & July \&, 1890 \\
\hline 94 & & - do & July s, 1890 \\
\hline & A & -...... do & Apr. 7,1891 \\
\hline & & Condine & Mav 5,1891 \\
\hline 173 & $d n$ & Cut-outs, mechanical & May 12, 1891 \\
\hline 4.5 & & zical, body-wear & Mar. 31, 1391 \\
\hline 98 & & Hea & June 23,1891 \\
\hline & & Regulato & Jan. 13,1891 \\
\hline & 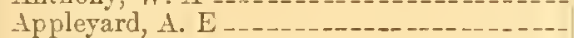 & Railways, track cleaners, wheel fenders & Dec. 30,1890 \\
\hline 910 & $\mathrm{~A}$ & Hea & June 30,1891 \\
\hline 134 & A & Lights, filamer & Oet. 14,1890 \\
\hline 169 & Ar & Optics. & Scpt. 23,1890 \\
\hline 350 & & Cond & Feb. 17,1891 \\
\hline 17 & & $\mathrm{~T}$ & Dec. 9,1890 \\
\hline 19 & & Medical and surgical, body-wear & Sept. 23,1390 \\
\hline & & Heaters & Feb. 24,1891 \\
\hline & & Alarms & Oct. 21,1890 \\
\hline 68 & As & Coin controlled apparatus & May 12,1891 \\
\hline 5 & & Separators .... & June 23,1891 \\
\hline 5 & & - & June 9,1891 \\
\hline & At & Railways, systems, overhead, trolleys ... & Aug. 26,1890 \\
\hline 8 & & & Sept. 9,1890 \\
\hline 107 & At & sitive feed, gear-clutch_. & Mar. 10,1891 \\
\hline & & Magneto-elcctric & Apr. 11,1891 \\
\hline & $\mathrm{B}$ & Annunciators & June 16,1891 \\
\hline 24 & Bailey, E. E. and G & Cut- & Scpt. 30,1890 \\
\hline 542 & G. WV $\ldots \ldots$ & Water distribution, siphons _-_- & July 8,1890 \\
\hline 611 & & Railways, systems, overhead, trolleys. & Aug. 5,1890 \\
\hline 187 & $\mathrm{Ba}$ & Insulators & Dec. 23,1890 \\
\hline & & Batteries, seco & Jan. 20,1891 \\
\hline 1 & $\mathrm{Ba}$ & Railways, syste & Map 26,1891 \\
\hline & $\mathrm{Bal}$ & Telephones, details & June 9,1891 \\
\hline 961 & & Railways, systems, overhead, trolleys & Oct. 7,1890 \\
\hline 86 & Baker, I. I & Electric locomotives & Nov. 18,1890 \\
\hline & Baker, I. F. anc & Railways, syste & Jan. 20,1891 \\
\hline $\begin{array}{l}3,100 \\
9,232\end{array}$ & $\begin{array}{l}\text { Baker, W. E. and Macloskie, C. H } \\
\text { Baldwin, C. W }\end{array}$ & $\begin{array}{l}\text { Railways, conductor supports and insulators } \\
\text { Air and gas engines, explosive. }\end{array}$ & $\begin{array}{l}\text { May } 26,1891 \\
\text { Oct. } 28,1890\end{array}$ \\
\hline
\end{tabular}




\begin{tabular}{|c|c|c|}
\hline No. & Name. & Class. \\
\hline 446,245 & Baldwin, G. R. & Electric locomotives \\
\hline 449,726 & Ball, C. M. and Norton, S - & Separators \\
\hline 449,307 & Ball, H. P & Conneetors -..-. \\
\hline 44,587 & Ball, R. E -.. & Regulators \\
\hline 51,798 & Butsley, C. H. and Porter, E. M . & Lights, sockets and keys ...... \\
\hline 41,919 & Banta, WV. E. & Connectors \\
\hline 0,614 & Barberie, E. T. and Des Brisay, $J$ & Cut-outs, mechanical _.................. \\
\hline 439,092 & Bardwell, A, F & Railways, systems, overhead, trolleys -.- \\
\hline 217 & Barrett, $\mathbf{A}_{-}-$. & Cut-outs, thermal \\
\hline 445,872 & Barrett, S. H .. & Batteries, secondary \\
\hline 132,927 & Barriett, S. L . & Lubricators, miscellaneous \\
\hline 846 & Barron, A. M & Telegraphy, circuit closers, thermal .... \\
\hline 452,561 & Bartholomew, I. H & Magneto-eleetric - \\
\hline 1,962 & Barton, E. M & Telephones, systems \\
\hline 49,651 & Bartsch, E--- & Electrodes \\
\hline 454,450 & Bassett, $\mathrm{N}$ & Car brakes \\
\hline 441,122 & Bassett, N. C- & Railways, systems, overhead, trolleys_..- \\
\hline 441,123 & Bassett, N. C. & do \\
\hline 442,472 & Batchelder, G. L . .. & Drop lights and hangers \\
\hline 294 & Bates, D. H. and Tan Hoereubergh, H... & Telegraphy, priuting \\
\hline 448,110 & Pates. L- & Magnetie locks and bolts \\
\hline 451,149 & Bates, P.S & Lights, incandescent, speeial features \\
\hline 440,753 & Bathurst, F ... & - \\
\hline 435,132 & Battershall, J. WV & Cut-outs, meehanical \\
\hline 352 & Battershall, J. IV & $\ldots \ldots$ do $\ldots . .$. \\
\hline 435,084 & H .... & Batteries, secondary, applications \\
\hline $5 \% 2$ & $\mathrm{R}_{-}$ & magnetie............ \\
\hline 611 & $\therefore V_{0}, j r_{-}$ & Applications, tools and machines \\
\hline 449,660 & Baxter, W., jr. & Motors ... \\
\hline 149,661 & Bixter, $\mathrm{W}, \mathrm{j} \mathrm{j} . .$. & Applications, tools and machines \\
\hline 419,662 & $\mathrm{~W}, \mathrm{jr}$ & $\ldots$ do $\ldots$..... \\
\hline 411,276 & Bayles, J. C - & Forging and welding -.- \\
\hline 441,277 & Bayles, J. C - & Mctal working, tubes \\
\hline 432,968 & , C. $\mathrm{H}_{--}$ & Adrertising, signs \\
\hline 438,982 & Beaeli, F, G -- & Telephones, systems \\
\hline 447,485 & Bene & do ... \\
\hline 437,767 & Beacl, R. H. & Lights, arc, positive feed \\
\hline 44,807 & Beach, R. H & Motors .......... \\
\hline 452,514 & Beck, C - & Alrertising, signs...................... \\
\hline 442,473 & Beers, C B. and Tu & Bells _-_. \\
\hline 433,390 & Bclding, IV. Ṡ_.. & Magneto-electrie ..-- \\
\hline$+33,391$ & Belding, W. S - & 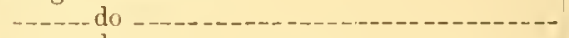 \\
\hline $\begin{array}{l}433,392 \\
433,393\end{array}$ & $\begin{array}{l}\text { Lelding, IV. S } \\
\text { Belding, IV S }\end{array}$ & 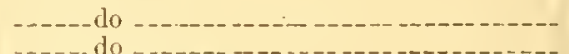 \\
\hline
\end{tabular}

Electric locomotives

Valre-controllers

Recorders

Regulators

Ear trumpets.

Lighting, systems

Applications, tools and machines

Telephoues, systems

Rheostats .

Teleplrones, systems

(1)

Electric loeomotives

Loeomotion - .

Railways, systems

Railways, systems, conduit-

Railways, systems, overhead -

Railways, systems, conduit ..........-....-. Feb. 17, 1891

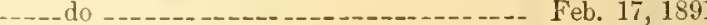

Railways, srstems, orerhead _.._-_.........' Apr. 7, 1891

Elect rie loeomotives................... June 16, 1891

_... do _._._. June 16, 1891

Railroads, systems, conduit_-_._._._._.... June 16, 1891

Lights, incindescent, special features.....-. July 1, 1890

Cut-outs, mechanieal .................. Aug. 5, 1890

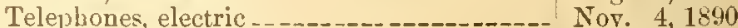

Telephones, magnetie

Televhones, eleetric -

Lights, sockets and keys _.............. Dec. 30, 1890

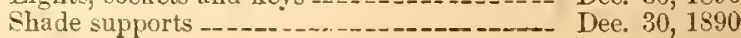




\begin{tabular}{|c|c|c|}
\hline Yo. & NAME. & ClaAss. \\
\hline $\pm 45,362$ & Bergmann, $\mathrm{S}$ & Cut-outs, mechanical _- \\
\hline 445.363 & Bergmann, $\mathrm{S}_{-}$ & Lights, sockets and kevs \\
\hline 445,364 & Bergmamn, S.... & - \\
\hline $445,36.5$ & Bergmann, S-_-_-.. & Connectors _.......... \\
\hline$\$ 6,180$ & Bergmann, S. and Klein, C. J & Lights, incandescent, special features \\
\hline 447,861 & Bergmann, S. & Cut-outs, meehanical _................. \\
\hline 436,205 & Berlin, C. E & Applications, general_................ \\
\hline 413,814 & Berne, A. IT & Railway signals \\
\hline 445.315 & Berne, A. W & Railway signals \\
\hline $433,2-29$ & Bernstein, A & Cut-outs, thermal \\
\hline 450,354 & Bertolette, D. A .. & Insulators........... \\
\hline 40,534 & Betteudorf, W. P. and J. W & Heaters ................ \\
\hline 454,794 & Bickford, J. H .......... & Connectors ........ \\
\hline 414,934 & Billberg, C. O. C. and Tinand, P. A. N & Motors, alternating \\
\hline $45.5,092$ & Billberg, C. O. C. and Winand, P. A. N & Cutouts, electro-magnetic-_-_-_-_-_.-. \\
\hline 449,400 & Bishop, J. D. & Conductors \\
\hline 443,199 & Blackwell, F. O & Electric locomotives \\
\hline 419,196 & Blackirell, F, O & Railwavs, systems, couduit ... \\
\hline 449,197 & Blackwell, F. O & Electric locomotives_..._._._. \\
\hline 452,160 & Blackwell, F. O .. & Railwayss, systems, conduit \\
\hline 452,422 & Blackmell, F. O & Electric locomotives \\
\hline 452,423 & Blackmell, F. O -- & 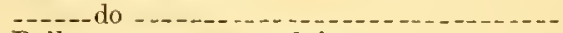 \\
\hline 454,023 & Blackmell, F. O _- & Railways, systems, conduit _. \\
\hline 444,687 & Blackwell, J. L & - \\
\hline 435,639 & Blades, H. H & Motors \\
\hline $43-, 640$ & Blades, H. H & Electric locomotives_-_._._- \\
\hline 435,641 & Blades, H. H & 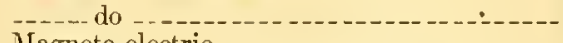 \\
\hline
\end{tabular}

\section{Magneto-electric -}

Railwars, systems, overhead, trollers

Blades, H. H

Blades, H. H

Blades, H. H

Blades, $\mathrm{H}$. H

Blades, H. H

Blair, J. and Hunter, A. G

Blaisdell, F. E

Blanchard, G. II

Blomgren, IT. A

Bock, L., jr

Booth, A. C

Bottome, T. D

Bottome, T. D

Fottome, T. D

Eottome, T. D

Bottome, T. D

Bornholdt, A

Bornholdt, A

Bossert, IV. F

Bradbury, J. Y. and Stone, E. J

Bradley, C. S

Bradley, C. S

Bradley, C. S

Bradley, C. S

Bradley, W. W. and Packer, IV. N

Brady, T. H

Brady, T. H

Brady, T. H

Branch, J. R

Branch, J. R

Braun, M. M

Bray, T. J

Breed, G

Bremer, IV. G

Bremer, W. G

Bremer, W. G

Bremer, W. G

Brennen, G. J

Brewer, H. J

Brewer, H. J

Brewer, H. .J

Brewer, H. J

Briggs, G. T

Brinkerhof, J. O. and Smith, MI. E

Motors

Magneto-electric

Motors

Insulating materials

Car brakes -.-----

Motors, reciprocating

Telegraphy, repeaters

Insulating materials

Cut-outs, thermal

Systems of distribution-

Lights, incandescent

$$
\text { -do }
$$

Cut-outs, thermal

Electric locomotifes

Batteries, secondary

Magneto-electric -

DATE.

Jan. 27, 1891

Jan, 27, 1891

Jan. $2 \bar{T}, 1891$

Jan. 2T, 1891

Feb. 10, 1891

Mar. 10, 1891

Sept. 9, 1890

Dec. 30, 1890

Mar. 17, 1891

July 29, 1890

Apr. 14, 1891

Nor. 11, 1890

June ?3, 1891

Jan. 20, 1891

June 30,1891

Var. 31, 1891

Mar. 17, 189

Nar. 31, 1891

Mar. 31, 1891

May 12, 1891

May 19, 1891

Nay 19, 1891

June 16, 1891

Jan. 13, 1891

Sept. 2, 1890

Sept. 2, 1890

Sept. 2, 1890

Oct. 7, 1890

Jin. 20, 1891

Nar. 3, 1891

May 12, 1891

May 26, 1891

June 16, 1891

Feb. 17, 1891

Nov. 11, 1890

Apr. 7,1891

July 22, 1890

Nov. 18, 1890

May 5, 1891

Nov. 4, 1890

Dec. 16,1890

Feb. 3, 1891

Feb. 3, 1891

Feb. 3, 1891

Nov. 25, 1890

Nov. 25, 1890

Nov. 4,1590

Feb. 17, 1891

Dec. 2, 1890

Qct. 21, 1890

Oct. 21, 1890

Oct. 28, 1890

Galvanometers

Clocks, electric

Supports and hangers

Block and tackle-

Insulators -

Railway signals, cab, sectional rail

Telegraphy, circuit closers, thermal

Towers, telegraph poles

Telegraphy, circuits and systems

Lights, sockets, and keys

Cut-outs, thermal

Railways, systems, closed conduit

Cut-outs, thermal

-.-...do -

Batteries, galvanic

Bateries, galvanic

------do -

Connectors

Motors

Batteries, galvanic

Meters

Telegraphy, fire, alarms aud extinguishers

Jan. 13, 1891

Jan. 13, 1891

Oct. 7, 1890

Dec. 9,1890

May 26,1891

Feb. 10, 1391

May 26, 1891

June 9, 1891

Nay 12, 1891

Sept. 2, 1890

Aug. 26,1890

Aug. 26, 1890

Nor. 18,1890

Dec. 30,1890

Dec. 30,1890

Oct. 14,1890

July 29,1890

Nor. 25,1890

Dec. 23,1890

A pr. 28, 1891

Mar 19, 1891

June 9, 1891

Oct. 28,1890

Feb. 10, 1891

Conductors, lead-covered................... Scpt. 30, 1890 


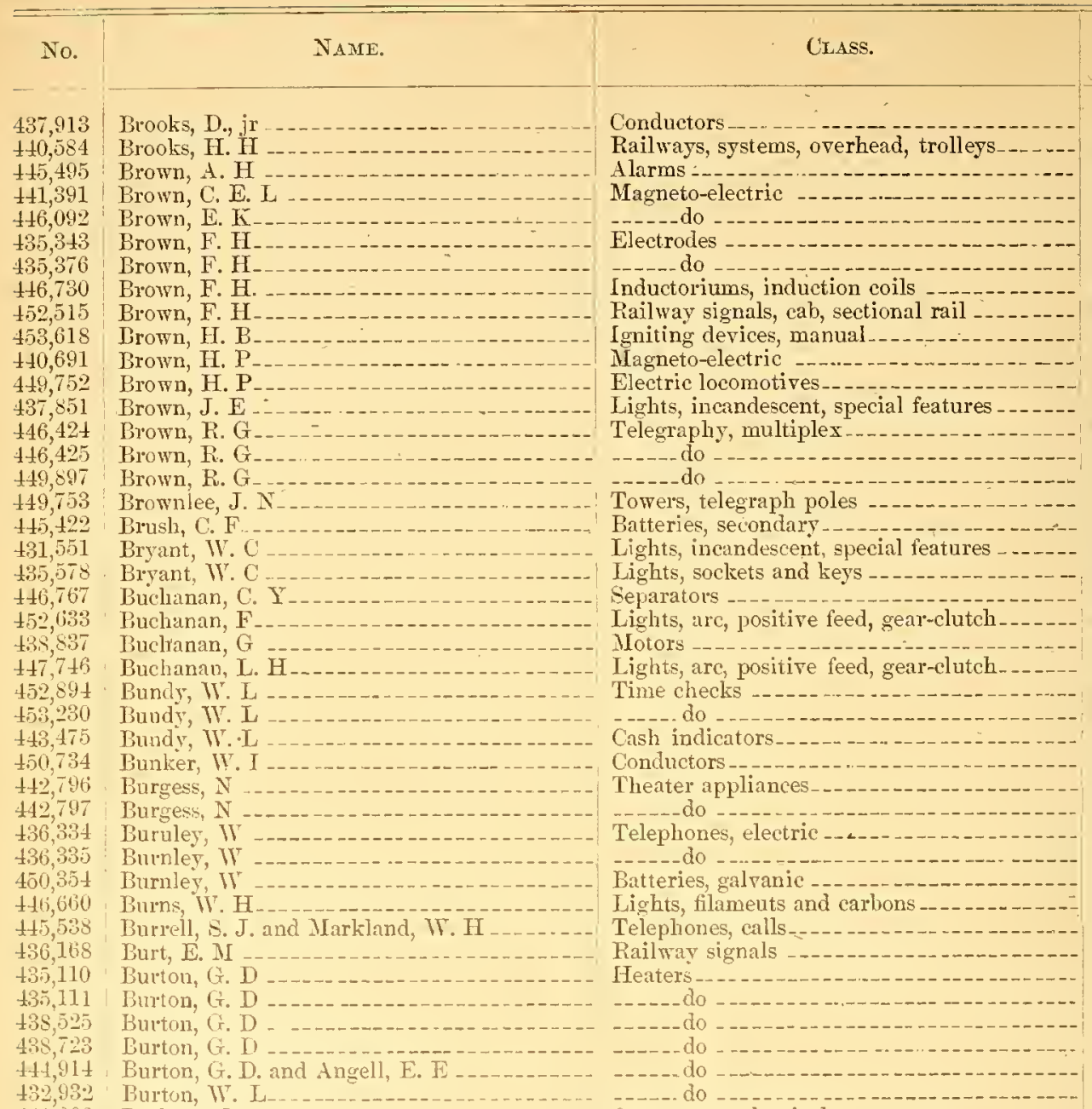

Burton, IV. I

$\begin{array}{ll}4 \pm 0,303 & \text { Bush, A. R } \\ 1 \pm 2,7 \pm 6 & \text { Busli, A. R - }\end{array}$

137,71 Butterfield, H. T

$\pm 17,127$ Buttcifield, H. R

$45.5,164$ Button, G. and IV reth, V. F

$411,7+5$ Butz, A. M

$\pm 3 \pm, 17$

440,428

435,011

431,240

434,501

$+15,648$

$449,03$.

449,036

411,396

449,731

433,671

436,110

147,023

$\pm \pm 5,317$

450,000

432,727

$44_{-19}^{2}, 90$

452,516

142,068

$4 \pm 1,933$

450,991

$4 \pm 2,856$

449,106

440,610

$\pm 52,030$

447,682

$\pm 38, \pm 04$

438,34

433,851

\section{Cady, IT. I}

Calioon, J. B. and Baker, I. F

Cairns, S. D

Cauderay, J

Cauderay, J

Cannell, IV

Capek, J. Y

Capek, J. I

Capps, F. L

Carlton, J. A.

Carpenter, C. E

Carpenter, C. E

Carpenter, C. E

Camenter, C. E

Carpenter, H. H.

Carl, C. P

Carn, R. S

Carroll, E. and Lambert, T. B

Cartwright, D. G. and Potter, J.

Cartwright, D. J

Cartwright, D. J

Carty, J. J

Carty, J. J

Casper, $\mathrm{L}$

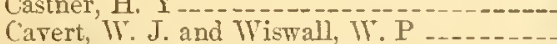

Chadbourne, A. H

Chaize, $\mathrm{N}$, and $\mathrm{J}$

Chamberlain, J. C
DATE.

Oct. 7,1890

Nov. 11, 1890

Jan. 27, 1891

Nov. 25, 1890

Feb. 10, 1891

Ang. 26, 1890

Aug. 26, 1890

Feb. 17, 1891

May 19, 1891

June 9, 1891

Nov. 18,1890

Apr. 7, 1891

Oct. 7,1890

Feb. 17, 1891

Feb. 17, 1891

Apr. 7, 1891

Apr. 7, 1891

Jan. 27, 1891

July 8, 1890

Sept. 2, 1890

Feb. 17, 1891

Nay 19, 1891

Oct. 21,1890

Mar. . 3, 1891

May 26, 1891

June 2, 1891

Dec. 23,1890

Apr. 21, 1891

Dec. 16,1890

Dec. 16,1890

Sept. 16,1890

Sept. 16, 1890

Apr. 14, 1891

Feb. 17, 1891

Feb. 3, 1891

Sept. 9, 1890

lug. 26,1890

Aug. 26, 1890

Oet. 1t, 1890

Oct. 21, 1890

Jan. 20, 1891

July 22, 1890

Nov. 11. 1890

Dec. 16,1890

Oct. 7, 1890

Feb. 24,1891

June 30, 1891

Dec. 2. 1890

Aug. 12, 1890

Feb. 17,1891

Aug. 26, 1890

July 1, 1890

Ang. 19, 1890

Feb. 3, 1891

Mar. 2t, 1891

Mar, 24, 1891

Nov. 25, 1890

Apr. T, 1891

Ang. 5, 1890

Sept. 9, 1890

Feb. 2t, 1891

Mal. 17, 1891

Apr, 7, 1891 July 22, 1890 Dec. 16,1890 May 19, 1891 Dec. $16 ; 1890$ Dec. 2, 1890 A pr. 21, 1891 Dec, 16,1890 Mar. 31, 1891 Dec. 16,1890 May 12, 1891 Mar. 3, 1891 Oct. 11,1890 Oct. 14,1890 Aug. 5, 1890 
No.

NAME.

$+17,230$

$+4,636$

48,603

453.978

447,939

432, 694

432.978

432.979

432,980

44,161

$48,-94$

$\$ 51,397$

452.71

44,330

$43+917$

450.927

40,013

431,104

454.934

$45+1,939$

$+4,54:$

439,929

$45 \div 214$

436,120

t5:-104

437,565

$449,2,2$

41.867

451,267

451,26 .

454,031

$433,4 \pm 5$

446,661

$45+253$

$45+926$

40,016

$47,7 \pm 5$

41427

440,390

451,614

47,505

434376

431,439

$435,2 \times 3$

435, 284

437.576

$43 \overline{0.51}$

439,47

439,478

142,954

$4+0,40$

42,800

45,220

$4+6,50$.

445,795

445,796

$44 .,-97$

445,798

45,699

$4.5,800$

445,801

445,502

$44 \overline{7}, 869$

$449,5,5$

449,59

410,596

$45-2,931$

452,932

452,993

431,555

439,431

$41,05 \%$

445,636

435,013

$4,50,213$

451,894

435,961
Chamberlain, J.

Chambers, J. C

Chaplin, E. D.

Chapman, X. S

Chapman, W. H

Chappell, G. H

Chase, H. A

Chase, H. A

Chase, H. A

Chase, H. A

Chase, H. A

Chase, H. A

Chase, .J

Chassagne, P. J

Chatard, F. E.

(hatfield, L. O

Cheever, J. H

Chenoweth, A. C

Chenoweth, A. C

Cheswright, H. T

(hild, C. T

Chisholm, D. J

Chubbuek, H. E

Church, A. II

(']ark, E

Clark, H. T

Clark, H. T

Clark, H. T

Clark, H. T

Clark, J. C

Clarke, $\mathrm{P}$

Clausen, W. H

Clereland, L. W

Cliff, E

Cobb, H. B

Cobb, H. B

Coburn, L

Cochran, G. II

Coffin, C. L

("offin, C. L

Coffin, C. I

Coffin, C. I

Coffin, C. I

Coffin, C. I

Coffin, C. I

Coftin, C. L

Cohlman, T

Colby, E. A

Colb $\mathrm{E}$ J

Cole, F. W

Cole, F. W

Cole, F. IV

Cole, F. IT

Cole, F. II

Cole, F. IT

Cole, F. W

Cole, F. IV

Cole, F. IV

Cole, F. W

Cole, F. WT

Cole, F. IV

Cole, F. W

Cole, F. IT

Colgate, A. E

Collier, J. TV

Collier, J. W

Collins, .J. J

Collins, IV. F

Collins, W. F

Collins, TV. $\mathrm{F}$

Compton, II. D
Clark, H. T
CLASS.

DATE.

Railways, systems, battery

Electrodes

Motors

Railway sionals, cab, sectional rail _._.__ June ?, 1891

Motors - .

Indicators... July 22, $1 \times 90$

Cut-outs, electro-magnetic_.___._._........ July 29, 1890

-_-_ do _-

Cut-outs, thermal _._._._._._._._._. July 29, 1890

Printing, hand stamps_-_-_._._.......... Jan. 27, 1891

Clocks, electric _.._._._._._._. Nar. 17, 1891

Telephones, switch-boards ................- Apr. 28, 1891

Telegraphy, fire _.................... May 26, 1891

Lights, arc, special features _..._._._._. . Jan. 6,1891

Lights, incandescent ........ Aug. 26,1890

Talve controllers _.......-_............ Apr. "21, 1891

Telegraphy, fire, alarms and extinguishers _- Nor. $t, 1840$

Insulating materials............. July 1, 1890

Conductors, underground ............ June 30,1891

-.....do - .

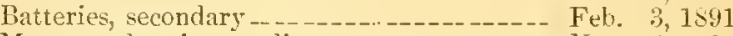

Magneto-electric, couplings -......... Nov, 1,1890

Motors _.................... Nar 12, 1891

Insulators_.___._._._. Sept. 9, 1890

Clocks, electric ....................... May 19, 1891

Conductors-_- Sept. 30, 1890

Cut-outs, mechanical _-_-_-_-_-_-_-_-- Mar. 31, 1891

C_._do _._. Mar. 10, 1891

_._._do _._._._. Apr. 28, 1891

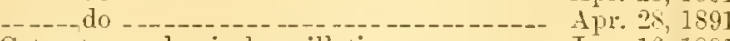

Cut-outs, mechanical, oscillating snap _.._-_- June 16, 1891

Recorders_- Aug. 5, 1890

Iagneto-electric, hand machines -....... Feb. 17, 1891

Lights, are, positive feed, cluteh _..._..._.. June 16, 1891

Cut-outs, electro-magnetic_._._._._._._._. June 30, 1891

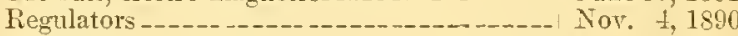

Telegraphy, cireuit closers _......_._._. Mar. 3, 1891

Drop lights and hangers..........._._._. Jan. ti, 1891

Vulcanizing apparatus.................. Nov. 11, 1890

Conductors, underground _._._._._._._._. May 5, 1891

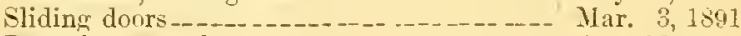

Batteries, secondary

Heaters

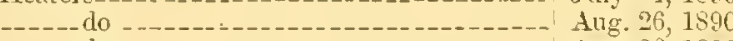

-

Sept. 30,1590

Sept. 30, 1890

Oct. 25,1890

Oct. 28,1890

Dec. 16,1890

Nor. 11, 1890

Dec. 16,1890

Jan. 27, 1891

Feb. 17, 1891

Feb. 3, 1891

Feb. 3, 1891

Feb. 3, 1891

Feb. 3, 1891

Feh. 3, 1891

Feb. 3, 1891

Feb. 3, 1891

Feb. 3, 1891

Mar. 10, 1891

Mar. 31, 1891

Mar. 31, 1691

Mar. 31, 1591

May 26,1891

May 26, 1891

June 9,1891

July s, 1890

Oet. 14, 1890

Nor. 18, 1890

Mar. 24, 1891

Ang. 26, 1890

Apr. 14, 1891

Mar 5, 1891

Sept. I, 1890 


No. NAME. Class.

\begin{tabular}{|c|c|}
\hline $64 t$ & G. \\
\hline 521 & G. H \\
\hline 11,060 & Conkling, $\mathrm{G}$ - \\
\hline 1,369 & Conkling, J. P \\
\hline 370 & Conkling, J. P \\
\hline, 104 & Conley, M $\ldots \ldots \ldots$ \\
\hline 482 & Conly, MIS. \\
\hline 403 & Conly, M. S-- \\
\hline 604 & Conner, IV. A \\
\hline 40 & Conner, IV. A \\
\hline $3+1$ & Conner, II. A \\
\hline 42 & Conner, IT. $\mathrm{A}_{-}$ \\
\hline 910 & Conradsou, C. MI - \\
\hline 561 & Conroy, T. and Fuhrman, L. P....... \\
\hline 94 & Cook, A. S \\
\hline & Cook, D. N \\
\hline 546 & Cook, W. S. and M. $\mathrm{C}_{-}$ \\
\hline 13 & Cook, W. S. and M. C. \\
\hline 45 & Conke, E. T. and Mackay, W. H - - \\
\hline 103 & Cooley, F-- \\
\hline 823 & Cope, T. J- \\
\hline $82+$ & Colve, T. J-- \\
\hline 79 & $\operatorname{lng}, F, G_{-}$ \\
\hline 0 & $\mathrm{~F} \cdot \mathrm{G}-\ldots$ \\
\hline 581 & Corning, F. G \\
\hline 2 & ng, F. $\left(r_{-}=\ldots\right.$ \\
\hline 808 & F. $(\dot{r}-$ \\
\hline 237 & Corning; $F .4+\ldots$ \\
\hline 801 & Cortese, MI -- \\
\hline 634 & W. 8 \\
\hline 5$]^{\circ}$ & Coughlilin, D. E--.--. \\
\hline$-1 \pm \bar{T}$ & Cox, H. B - \\
\hline $4: 25$ & Cox, II. B - \\
\hline+429 & 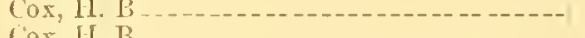 \\
\hline 0.558 & 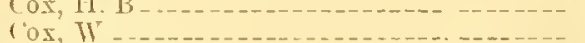 \\
\hline
\end{tabular}

431,649 434,382

438,140

435,2206

$438,0.30$

$4+2,423$

$4 \pm 1,392$

447,279

$4.50,834$

458,995

446,732

435,536

$4+5,25$.

449,209

$+5,2,565$

440,789

$4+5,707$

$44,7.28$

433,619

Cram, TV. E

Crampton, 'T.

Crane, M. G

Crane, M. G

Ciane, 1l. (

cane, M. Ir. and Cole, F. II

Cregier, N. B

reighton, $\mathrm{T}$ -

Clisi, J. A. and

Crockett, C. H. and Dedrick, L. C

Crosby, E. H

Crovidus, IV. A

Crowdus, II. A

Culver, F.

Cummings, G. P

Cumningtou, J. G. I

Cunynghame, H. H., Rawson, F. L. and IVoodhouse, O. E

Currie, s.

currie, S. C. C

Curie, S. C. C

Currie, S. C. C

Currie, S. C. O

Currie, S. C. C

Currie, S. C.C

Currie, S. C. C

Currier, J. B. aud Rice, D. H

Curtis, H. N

Curtis, IV. B. and Himrod, C ...............

Curry, $\mathrm{ll}^{\circ}$

Cushing, B. D

Cutler, H. H

Cutler, H. H
Storepipe thimbles

Magreto-electric

Separators

-

Indicators

Railway siguals

Conducto

Conductors, lead-covered do

Electric locomotives

Steam valves, throttle

Uagneto-electric -

Railways, systems, overhead, trolleys

Railway signals

Telegraphy, railway-car

Lighting, systems

Lamps and gas fittings, reflectors

Conductors, underground

Railways, systems, battery

-1
-10

Railway signals

Telcphones, uagnetic

IT earing, stopping -

Batterics, thermal

Currie, S. C. C

Curtiss, G. F-

Cutler, J. I

Metal, curred surfaces
-

Annunciators:

-

Lights, arc, gravity feed, clutch

Telcoriphy, firc Bells

Annunciators

Telcgraphy, railway-car

Insulators

Medical and surgical, body-wear

Electric locomotives

Telegraphy, keys .

Batterics, galvanic -......

Railwars, srstems, overhcad

Batterics, galvauic

Cut-onts, mechanical

Railwars, systems, orerhead

Cut-outs, thermal

Galvanouetcrs

Electric locomotives

Applications, tools and machines

Lighting, srstems, secondary battery

Galvanometers

Galvanometers

Batteries, secondars

_... A do 21, 1891

June 9, 1891

Telephones, calls _..................... Feb. 17, 1891

Railways, systems, conduit_............ Sept. 2, 1890

Lamp shades _. Jan, 27, 1891

Cut-outs, thermal _...................... 31, 1891

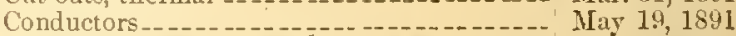

Railwars, srstenı, orerhead, trolleys _...... Nov. 18, 1890

Igniting derices, automatic _..._._._-... Feb. 3, 1891

Teledo - Feb. 3, 1891

Sept. 9, 1890
Dec. 2,1890

18, 1890

Арг. 28, 1891

sept. 2,1890

Mar. 17, 1891

May 12,1891

May 12,1891

Dec. 16,1890

Mav 19, 1891

7,1891

July 22, 1890

ar. 31, 1891

30,1590

Aug. 19, 1890

1u. 1890

ct. 1890

Sept. 2, 1890

July 2\%, 1890

Aing. 19, 1890

19,1890

Mar. 3, 1891

Feb. 3, 1891

May 12, 1891

IIay I', 1891

Apr. 21, 1891

Iar. 10, 1891

Oct 11,1890

Sept. 30,1890

Jan. 27, 1891

pr. 21, 1891

Aug. 5, 1890

pr. 14, 1891

Oct. 14,1890

Oct. 14,1890

Oct. 14,1890

Aug. 5, 1890 
No.

NANE.

$\pm 4+37 i$

45,310

431,035

437.650

451.108

453,466

$436,7,7$

453,337

433,981

$453, \$ 58$

$+50,5 \pi$

$449, \pm 68$

451,658

$+41,4-4$

451,649

$4.51,6.56$

439,345

49.362

435, 213

$432,02-2$

$45+, 17$

436,219

434,922

$435 \overline{1}, 114$

40,294

140,627

431,181

$+31,65$

40,766

40,76 .

440,768

450,615

452,571

452,572

152,37

$4.52,574$

$443, i+6$

431,564

141,967

445,360

449,318

42,074

437,412

435,558

449,108

$4.54,990$

433,560

$48,7,27$

$43.5,6+2$

435,64 .

435,64

436,12 .

436,519

438,406

435,407

438,405

438,409

$439,57 \mathrm{i}$

439,867

411,401

41,828

412,365

443,748

443,749

445,770

$4+5,-7$

46,703

447,104

$48,5 i 9$

449,404

451,239

451,240

$4.52,095$

452,775

431,879

$433,4 \tilde{1}$

$+48,322$

Cutter, G

Daft, L

Datuon, G. I

Durling, E. A .

Daridson, B. E

Daris, C. E

Daris. E

Daris, J-

Davis, J. Y

Daris, M. G

Dawes, $A$

Darton, C. A

Dean, F. II

Dean, J. H.

De Castro, A-

Decker, P

Deeds, H. WT

Deerow, IT. E

De Ferranti, S. Z

De Ferranti, S. Z

De Ferranti, S.

Delany, P. B

Delany, P. B

Delans. P. B

Delany, P. B-

Delany, P. B

Delany, P. B

Deming, $\mathrm{E}$

Deming, $\mathrm{E}$

Deming, E

Deming, E

Dempster, J. T

Denison, S. P

De Peralta, C. G

De Place, I

Depp, O. J

Deprez, M.

Desant, T. F. Z

Desant, IT. F. Z

Des Brisay, J

Detwiller, W. B

Derrey, M. II

Dewer, M. II

Dewey, M. II

Dewey, MI. IV

Dewer, MI. W

Dewey, MI. II

Derey, M. W

Dewey, M. II

Dewey, M. II

Dewey, M. II

Derey, M. W

Dewey, M. W

Dewey, M. II

Dewey, M. W

Dewey, M. IV

Dewey, MI. IV

Dewey, M. W

Dewey, M. W

Dewey, M. W

Dewey, M. II

Dewey, M. II

Dewey, M. IV

Dewey, II. IV

Dewey, M. IV

Dewey, M. W

Dickson, C. T.

Dickerson, E. N

Dickerson, E. N

Digeon, I
Cutter, G. and Armstrong, C. G

Danals, C. A. and Perry, W. O

Davis, H. and Stokes, A. H

Deacon, S. and Wightman, M. J

Depp, O. J. and Munn, S.J
Class.

Date:

Conductors, underground

Cut-outs, mechanical

Cut-outs, electro-magnetic

Magnetic locks and bolts

Drop lights aud hangers

Ala rms, burglar

Railway signals

Cut-outs, thermal

Telephones, electric

Magneto-electric

Induction coils

Scale beams

Telegraphy, fire

Drop lights and hangers

Valre controllers

Lights, sockets and keys..

Electric locomotives

Motors

Time alarms

Induction coils

Applications, general

Valve-controllers

Telegraphy, fire

Inductoriums, transformers, stationary -

Systems of distribution

Meters

Bells

T'elegraphy, multiplex

-

Annunciators

Telegraphy, railway-car

Railway signals, cab, sectional rail

$$
\text { do }
$$

Jights, sockets and keys

Sawing metal.

Batteries, galvanic

Applications, general

Cut-outs, mechanical

Ruilway signals

Rheostats -----
Railway signals

Cash iudicators

Cut-outs, mechanical

Letter boxes

Heaters

Electric locomotives

Heaters

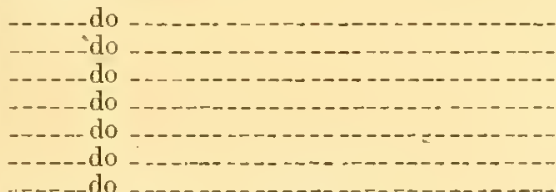

Applications, tools and machines

Railways, systems, circuits, series

Heaters

Railways, systems do

Heaters

$$
\text { do }
$$$$
\text { do }
$$

Applications, general

Heaters

$$
\text { -.--- do }
$$

-----do

$$
\text { do - }
$$$$
\text { do }
$$

Railways, systems, double

Telephones, details

Systems of distribution

Batteries, thermal.

Telephones, systems

Jan. 6,1891

Jan. 27,1891

July 1, 1890

Oct. 7, 1890

Apr. 28, 1891

June 2, 1891

Sept. 23, 1890

June 2, 1891

Aug. 12, 1890

June 9, 1891

Apr. 14, 189

Mar. 31, 189

Lay 5, 1891

Jan. 13, 1891

May 5, 1891

May 5, 1891

Oct. 2s, 1890

Iar. 31, 1891

Aug. 26, 1890

July 10,1890

June 23, 1891

Sept. 9, 1890

Aug. 26, 1890

1ug. 26, 1890

Nor. 11, 1890

Nor. 18, 1890

July 1,1890

July \&, 1890

Not. 18, 1890

Nor. 18, 1890

Nov. 18, 1890

Apr. 21, 1891

May 26,1891

May 26, 1891

Uav 26, 1891

May 26, 1891

Dec. 30,1890

Juls \&, 1890

Dec. 2, 1890

Feb. 3, 189

Mar. 31, 1891

Dec. 16,1890

Sept. 30, 1890

Sept. 2, 1890

Mar. 31, 1891

June 30,1891

Aug. 5, 1890

July 22, 1890

Sept. 2, 1890

Sept. 2, 1890

Sept. 2, 1890

Sept. 9, 1890

Sept. 16,1890

Oct. 1t, 1890

Oct. $1 \frac{1}{1}, 1890$

Oct. 14,1820

Oct. $1 \frac{1}{1}, 1890$

Oct. 28,1890

Nor, 4, 1890

พัง. 25, 1890

Dec. 2, 1890

Dec. 9,1890

Dec. 30,1890

Dec. 30, 1890 


\begin{tabular}{|c|c|c|c|}
\hline No. & TAYIE. & Class. & Date. \\
\hline & Dillon, L. IT & Lights, incandescent, special features & July 1,1890 \\
\hline 454,166 & Dillon, $\mathrm{T}$ & Street lamp hangers & June 16,1891 \\
\hline 438,773 & Dinn, P. J- & Lamp posts . ......... & Oct. 21,1890 \\
\hline 431,447 & Dixon, T. S. 1 & Batteries, secondary -- & July 1,1890 \\
\hline 452,992 & Doblie, R. S. & Electric locomotives & May 26,1891 \\
\hline$+45,442$ & Doggett, J - - & Telegraphy, relays and sounders. & Jan. 27,1891 \\
\hline 431,565 & Donnelly, $A$ & Marine torpedoes ................ & July 8,1890 \\
\hline 446,831 & Donovan, $\mathrm{T}$. J & Heaters ...... & Feb. 17,1891 \\
\hline 402,599 & Dooley, T. B. & Miscellaneous compositions & May 19,1891 \\
\hline 452,072 & Doucet, S. J & Railway signals, cab, sectional rail _- & May 12,1891 \\
\hline 431,883 & Donglass, L. & Phonographs _._. & July , 8, 1890 \\
\hline 445,931 & Donse, T. $\mathrm{H}$ & Telegraphy, circuit closers, thermal & Feb. 3, 1891 \\
\hline$+51,996$ & Dow, II. E & Drop lights apd hangers & May 12,1891 \\
\hline 451,997 & Dow, II. E - & Cut-outs, thermal -........ & May 12,1891 \\
\hline 446,130 & Trowney, G. II & Lightning rods _.. & Feb. 10,1891 \\
\hline $43 \div, 988$ & - - & Motors _........ & July 29,1890 \\
\hline 436.560 & Doyle, & Bells & Sept. 16,1890 \\
\hline$+41,293$ & Doyle, J -. & Rheostats - - & Nov. 25,1890 \\
\hline 440,699 & Dressler, C. E & Mntor & Nov. 18,1890 \\
\hline 00 & Hressler; C. E & Inductoriums, transformers, rotary & Nor. 18,1890 \\
\hline 442,173 & Jressles; C. E & Jagneto-electric _........... & Dee, 9,1890 \\
\hline 145,499 & - - - - 1- & Motors & Jan. 27, 1891 \\
\hline 439,724 & Drew, C. IV. and Francis, E. R .......... & Heaters ............ & Nor. 4,1890 \\
\hline $45+, 979$ & Drew; $\mathrm{C} . \mathrm{W}$. and Francis, E. R & --.---do & June 30,1891 \\
\hline 451,300 & Inew, H. P & Lights, incandescent, special fea & A pr. 28,1891 \\
\hline$+3,584$ & - & Registers _. & Aug. 19,1890 \\
\hline 431,256 & - n- & Electric locomotires -- -- & July 1,1890 \\
\hline 453,041 & Drresdale, W. A & Lights, incandesecnt, special feature & May 26,1891 \\
\hline 442,868 & ...- & Conductors, underground $\ldots \ldots \ldots$ & Dec. 16,1890 \\
\hline 105 & n-n & Clocks, electric & Feb. 24,1891 \\
\hline $114,7,7$ & - n-n & Magneto-elcetric --_--.-- & Apr. 7,1891 \\
\hline $4: 37,293^{\circ}$ & . n & Vending apparatus _........ & Sept. 30,1890 \\
\hline 435,559 & 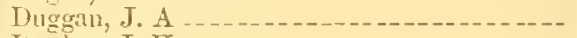 & Railways, systems, overliead, trolleys & Sept. 2,1890 \\
\hline $434,14 t$ & (1) & Insulators. & Aug. 12,1890 \\
\hline 454,536 & 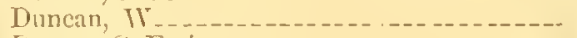 & Rililways, syster & June 23,1891 \\
\hline 16 & Dutton, C. E., & Batteries, galranic & Dec. 9,1890 \\
\hline $437,1 \times 3$ & 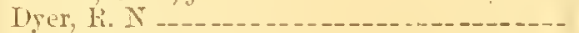 & Weters & Sept. 30,1890 \\
\hline 439,838 & - & Clocks, electric & Nor. 4,1890 \\
\hline 433,523 & Fiston, I. II & Magneto-electric -- & Ang. 5, 1890 \\
\hline 436,733 & Easton, J. II & Insulating materials. & Sept. 16,1890 \\
\hline $\pm 45,5+4$ & Eastnn, J. IV & Lights, arc, gravity feed, gea & Feb. 3,1891 \\
\hline $4+8,+1 \mathrm{~s}$ & Easton, .T. IV & - do _.... & Mar. 17, 1891 \\
\hline 449,902 & Easton, ]. II & Drop lights an & Арг. 7,1891 \\
\hline 449,903 & Easton, J: II & Telegraphy, lightning arresters. & Apr. 7,1891 \\
\hline 449,407 & Easton, J. IV & 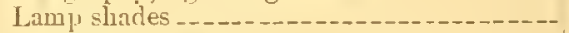 & Mar, 31, 1891 \\
\hline 453,323 & Ebert, IV. $\mathrm{H}_{-}$ & Liglitiug, syste & June 2,1891 \\
\hline 438,592 & Eckert, W. H. and Gregrory, W. H & Crinduciors-. & Oct. 14,1890 \\
\hline 440.575 & Eckert, W. H. and Gregory, W. H $\ldots$ & Lighting, systc & Dee. 9,1890 \\
\hline 448,325 & Edgerton, E. M & Time alalms ..... & Mar. 17,1891 \\
\hline $432,-148$ & Edgerton, $\mathrm{N} . \mathrm{H}$ & Nagneto-electric_.. & July 22,1890 \\
\hline 42,870 & Eilgerton, $\mathrm{N}$. & 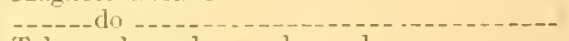 & Dec. 16,1890 \\
\hline $43 \pm, 585$ & EH & Telegraplụ", relays : & Lug. 19,1890 \\
\hline 435,586 & Edison, T. A. & Prro-magneto el & Aug. 19,1890 \\
\hline 587 & Edison, T. A & Bitteries, thermal _- & Aug. 19,1890 \\
\hline 434,588 & Edison, T. A. and Thickion, IT. K. L.. & Separators ... & Aug. 19,1890 \\
\hline 589 & Edison, T. A & Electric locom & Ang. 19,1890 \\
\hline 435,687 & Edison, T. A & Batteries, secondary, appli & Sept. 2,1890 \\
\hline$+35,688$ & Edison, T. A & Batteries, galvanic - & Sept. 2,1890 \\
\hline 689 & Edison, T. & Telegraphy, du & Sept. 2,1890 \\
\hline 435,690 & Edison, $T . A$ & IIagneto-electri & Sept. 2, 1890 \\
\hline 127 & Edison, T. A & Tran-mission of pow & Sept. 9, 1890 \\
\hline 437,422 & Edison, T. A- & Telegraphy, cirenits & Sept. 30,1890 \\
\hline & Edison, T. I & ('Intches, intermittent-grip & Sept. 30,1890 \\
\hline & Edison, T. & Lights, incande & Oct. 14,1890 \\
\hline 438,299 & Edison, T. A- & Liglits, filaments and carbon & Oct. 14,1890 \\
\hline 301 & Exison, T. A. & Cut-outs, electro-magnetic-. & Oct. 14,1890 \\
\hline & Edison. T. A- & Magneto-electric & Oct. 14,1890 \\
\hline 303 & Edison, T. A & Lights, are, positive fee & Oct. 14,1890 \\
\hline $30 \pm$ & Edison, T. A- & Telepliones, calls & Oet. 14,1890 \\
\hline .305 & Edison, T. A... & Cut-outs, thermal - & Oct. 14,1890 \\
\hline & Edison, T. A. and & Telephones, electric & Oct. 14,1890 \\
\hline & Edison, T. A & Lights, incandescent & Oct. 14,1890 \\
\hline 308 & Edison, T. A & Systems of distributio & Oct. 14,1890 \\
\hline 309 & $\begin{array}{l}\text { Edisou, T. A } \\
\text { Edisou, T. A }\end{array}$ & Insulating materials & Oct. 14,1590 \\
\hline $\begin{array}{l}310 \\
389\end{array}$ & Edisou, & $\begin{array}{l}\text { Lights } \\
\text { Lighti }\end{array}$ & $\begin{array}{ll}\text { Oct. } & 14,1890 \\
\text { Oct. } 28,1890\end{array}$ \\
\hline $\begin{array}{l}432,389 \\
439,390\end{array}$ & Edison, T. A & $\begin{array}{l}\text { Lighting, systems } \\
\text { Lighting, systems, secondary battery }\end{array}$ & $\begin{array}{l}\text { Oct. } 28,1890 \\
\text { Oct. } 28,1890\end{array}$ \\
\hline
\end{tabular}


No. NAME.

Class.

DATE.

439,391 Edison, T. A

439,392 Eilison. T. A

439,393 Edison, T. A

44.530 Edison, T. A

446,666 Edison, T. A

$4 \pm 6,667$ Edison, T. A

446,668 Edison, T. $A$

446,669 Edison, T. A

$4 \pm 8,778$ Edison, T. A

45:-913 Edison, T. A

453,601 Edison, T. A

$45+262$ Edison, T. A

$454,55.5$ Edison, T. A

$4 \pm 3,360$ Edson, J. B

453,111

$447,17 \overline{7}$

46,704

451,208

449,847

434,55

$431,55 \mathrm{~s}$

443,671

$45,3 \geq 6$

418,831

450,367

$4.51,320$

453,167

$4.5, \div 07$

$45+, 336$

$45+4,+62$

436,936

437,754

$446,6-11$

$443,6+2$

44,879

44,977

445,136

439.726

435,456

$+35,45$

46,284

443,991

453,693

$4.5,773$

433,724

$439,7+6$

441,753

$443,17 \pm$

443,503

451,177

437,632

451,921

$4.54,724$

432,397

435,311

$43 \times, 312$

435,313

441,403

441,542

411,543

434,590

44,486

448,323

$4+10,023$

440,024

450.187

443,915

448,916

418,911

$143,13 \mathrm{t}$

14:3,135

$443,13 f_{j}$

404,339

$43.5,292$

445,715

445,716
Edsou,

Edwards, Ti. C

Edwards, II.

Eggers, F. F

Eickemeyer, $\mathrm{R}$

Eickemeyer, R

Eickemeyer, $\mathrm{R}$

Eickemeyer, $\mathrm{R}$

Eickemeyer, R.,

Eickemerer, R

Eiveukuth, J. TV

Einstein, J. and Kornprobst, S..........

Elfering, J. H

Elieson, C. 1

Elkins, $\mathrm{C}$

Elkins, II. H

Elkins, TI. H

Elliott, G. F

Elliott, W. R

Elliott, II. P

Ellis, A. I

Ellis, S. R

Ells, J. IT

Ely, E. S -

Emmet, WV. Le R

Emmet, IT. Le $\mathrm{P}$

Emmet, W. Le R

Emmet, WV. Le R

Emmet, WV. Le $\mathbf{R}$

Enımner, J., jr

Emmner, J., ji

Emmner, J., jr

Enholm, O. A

Enholm, O. A

Enholm, O. A

Enholm, O. A

Enholm, O. A

Entz, J. B

Entz, J. X

Entz, J. B

Entx, J. B. and Phillips, W. A

Entz, J. B. and Phillips, W. A

Erlwein, $G$

Erlwein, G

Erlwein, G

Erlwein, G. and Heller, F. G. A

Essick, S. V. B.

Essick, S. V. B

E-sick, S. Y.

Etheridge, $\mathbf{H}$

Etheridge, $\mathrm{H}$
Eickeneyer, $\mathrm{R}$

Eickemever, $\mathrm{B}$

Eickemeyer, $\mathrm{R}$

Essick, S. V. B
Condnctors, undergronnd

Lighting, systems

Lights, filaments and carbons

Lights, incandescent

Lighting, systems, secondary battery

Electric locomotives

Liglits, are, gravity feed, clutch

Lights, flaments and carbons

Rililways, systems, circuits, multiple arc

Telegraphy, circuits and systems

Telegrapliy, duplex

Telegraphy, multiplex

Lights, filaments and carbons

Lights, incandescent

Recorders

Cut-onts, thermal

Lighting, systems

Separators

Dental instruments

Galvanometers

-....--do

Electric locomotives

Motors, alternating

Railways, systems, overhead

Magneto-electric

Motors, reciprocating

Electric locomotives

Rheostats

IIagneto-electric

Applications, tools and machines

Air and gas engines, explosive.

Weter's

Annunciators

Meter's

Insulators

Lights, arc, gravity feed, clutch

Store service, gravity --.-..-
Railways, systems, closed conduit

Regulators

Theater appliances

Lights, arc, positive feed, gear-clutch

Indicators

Door bells

Rivilways, systems, overhead

Bailways, systems, overhead, trolleys

Railways, conductor supports and insulators

IIagneto-electric

Motors

Batteries, galvanic

Magneto-electric

Insulating materials

Glass and pottery, jars

Insulating materials

Balteries, galvanic

Systems of distribution

Lights, arc, gravity feed, clutch

Magneto-electric-. do

Railways, syostems

Batteries, secondary do

do

Telegraphy, printing

-

Telegraphy, relays and sounders

Telegraphy, automatic -....... do

do
Batteries, secondary

Lights, filaments and carbons
Oct. 28,1890

Oct. 28, 1890

Oct. 28, 1890

Jan. 1\%, 1891

Feb. 17, 1891

Feb. 17, 1891

Feb. 17,1891

Feb. 17, 1891

Mar. 24, 1891

Mar. 24, 1891

May 26, 1891

June 2, 1891

June 16,1891

June 23,1891

Dec. 23,1890

Nay 26,1891

Feb, 24, 1891

Feb. 17, 1891

Apr. 28, 1591

Apr. 7, 1891

Ang. 19, 1890

Aug. 19, 1890

Dec. 30,1890

Max. 17, 1891

Nar. 24, 1891

Apr. 14, 1891

Apr. 28, 1891

May 26, 1891

June 16,1891

June 16, 1891

June 23, 1891

Sept. 23, 1890

Oct. 7,1890

Feb. 17, 1891

Dec. 30,1890

Jan. 20, 1891

Jan. 20, 1891

Jan. 20, 1891

Nov. 4, 1890

Sept. 2, 1890

Sept. 2, 1890

Feb. 10, 1891

Jan. 6, 1891

June 9, 1891

Feb. 3, 1891

Aug. 5, 1890

Nov. 4, 1890

Dec. 2, 1890

Dec. 23,1890

Dec. 30,1890

A pr. 28, 1891

Oct. 7,1890

May 12, 1891

June 23, 1891

July 15, 1890

Oct. 14,1890

Oct. 14,1890

Oct. 14,1890

Nov. 25, 1890

Nov. 25,1890

Nov. 25, 1890

Aug. 19, 1890

Jin. 13, 1891

Mar. 17, 1591

Nov. 4, 1890

Nor. $t, 1890$

June 30, 1891

Mar. 24, 1891

Mar. 24, 1891

Mar. 24, 1891

Dec. 23, 1890

Dec, 23, 1890

Dec, 23,1890

June 16,1891

Aug. 26, 1890

Feb. 3, 1891

Feb. 3, 1891 
450,099 Fahnehjelm, O. B

454,038 Fanning, J. J.

445,051 Faries, R. P

$448,6 \pm t$ Farmer, M. G

444,108 Farnham, I. H

451,922 Fal'nham, I. H

448.711 Farnham, I. H

$+39,238$

438,579

443,636

431,185

431,186

431,718

$452,37.5$

443,478

$454,90 \pm$

439,459

452,494

$453,7+2$

432,521

433,120

436,408

$446,26(j 6$

$448,39 ?$

439,347

$4.44,593$

$449,67 \cdot-2$

$430,40:$

442, $04: 2$

$442,04: 3$

$442,(1.14$

449,245

$44 \div .0201$

442030

$452,: 317$

444,217

$445,5.1$

$434,(12)$ liteh II. I

412060 Fisz-ficrald

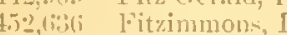

(5:, , 5

$43 !$

$43: 13$

$451,-30$

44,

431

$431, \cdots+1$

430,400

$43 \%, 4()$

$433,4(1)$

134,180

434,18

434,261

$435,+40$

$45+, 643$

to.),

$451,81$.

449,15

445,59

433,593

434,017

$434, \pi$

447,768

435,296

439,29 .

$44^{2}, 5 i 7$

410,42

440,425

$440,3.57$

441,793

441,794

452,934

$4.2,93.5$

Fanre, C. A

Favor, L. B

Favor, L. B

Flet cher, J. R

Ferrell, I. M

Fessenden, R. 1

Field, $5 . \mathrm{D}$

Field, S. D

Field, S. D

Field, S. I

Field, $\mathrm{S}$

Fink, IY. MI

Finncy, G. S

inney; $\mathrm{r}$.

Fisher, 13. II

Fisher, F. I:

Vischer, J. 1 :

Fisk, P. II

Fiske, B. A

vitzimmons,

Flage, 1. J

Flenminger, ()

Flower. II. I

Fucer,

ronte, T. II

Foote. T. . II

Forte, T. II

Poute T.

Foote, 'T. II

Foote, G. I.

Ford, IY. I

Forgason, II.

Foster, H. A

Fottrell. J

Fowler, H. F

Fox, E. M -

rancis, J.

Fraser, IV. J

Fraser, II. S

Freeman, IY.

Freeman, W. $K$

Freeman, WV.

Freeman, W. $\mathrm{K}$

Freeman, W. K
Faunce, A. B. and Cabell, S. G

Feary F. T. and Speicher, J--

Finn, II. J. and ( tarner, J. A

Foster, C. and Beris, W. H
Gas burners, incandescent

Lighting, systems

ms, transformers, stationary

Telephones, systems

Telephones, toll

Dental instruments

Incrustation

Meters

Cut-outs, thermal

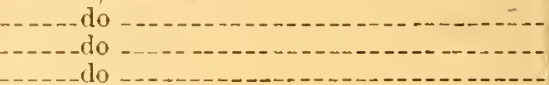

Telegraphy, fire

Cut-outs, thermal

Lightning rods

Lights, incandeseent

Cut-outs, thermal

Telephones, electric

Magneto-electric

C'ut-outs, thermal ---

Medical and surgical, body-mear

IBatteries, galvanic

Telegraphy, railway-ear

Separators

-

liegulators -..

Innunciators

cut-outs, meclianical

lights, incandescent, special features

Telegrapliy, printing

Distance instruments

Drop lights and hanger

Liurlit incendescent, special features -

Batteries, seeondary

Telephones, elcetrie

Lights, inciudescent

Railwavs, systems, battery

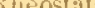

Towers, telegrapla poles

Ruilways, systems, overhead _............

Iagnetic locks and bolts

Narms

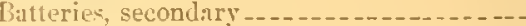

Motor:-

Electric locomotives

Totors .........

Thegulators _._.

Telephones, calls, step-by-step _..........

Telephones, srstems, automatic

Railwars, systerus, closed eonduit .........

Lights, arc, gravity feed, clutch .

I.ights, arc, special features ................

Railway, conductor supports and insulators Insulating materials .-

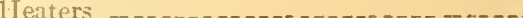

Thermometer

Telephones, systems

Magneto-elcetric -

Recorder

Lamp shades

Magneto-electric-_-_.

-----do

Regulator

Magneto-eleetric.

Neters
Apr. 7,1891

Jan. 20, 1891

Mar. 24, 1891

Jan. 6, 1891

May 12, 1891

Mar. 24, 1891

Oct. 28,1890

Oct. 14,1890

Dee. 30, 1890

July 1,1890

July 1, 1890

July 8, 1890

May 26, 1891

Dec. 23,1890

June 30, 1891

Aug. 5, 1890

May 19,1891

June 9, 1891

July 22,1590

July 29, 1890

Sept. 16, 1890

Feb. 24, 1891

Mar. 17, 1891

Oct. 2S, 1890

Aug. 19, 1890

Apr. 7,1891

July 22, 1590

Dce. 16,1890

Dec. $\mathbf{1 6}, 1890$

Dec. 16,1890

Mar. 31, 1891

Dec. 2, 1590

Dec. 2, 1890

May 19, 1891

Apr. 14, 1891

Ji1. 6, 1891

Feb. 3, 1891

Alug. 26, 1590

Der. 16, 1890

May 19, 1891

June 2, 1891

May 26, 1891

Oct. 28, 1890

July 15, 1890

Niay 5, 1891

June 30,1591

Feb. 24, 1591

July S, 1890

July 1, 1590

July 29,1590

July 29, 1890

July 29, 1890

tug. 12, 1890

Aug. 12,1890

Aug. 12, 1890

Sept. "2, 1890

June 23, 1891

Aug. 26, 1890

Nay 5, 1891

Mar. 10, 1891

Mar. 31, 1891

Feb. 3, 1891

Aug. 5, 1890

Aug. 12, 1890

Aug. 19,1890

Mar. 10, 1891

dug. 26, 1890

Oct. 28, 1890

Dee. 9, 1890

Nor. 11, 1890

Nor. 1I, 1890

Nor. 11, 1890

Dec. 2, 1890

Dee. 2, 1890

May 26, 189$]$

May 26, 1891 


\begin{tabular}{l|l|l}
\hline No. & NAME. & Class. \\
\hline
\end{tabular}

452,936 Freeman, W. K

45:2,937 Freeman, W. K

$43:, 234$ Frey, A. C

$433,89 . \quad$ Frey, C. P

118,331 Frietseh, K. G

451,037 Frost, E. J

435,58 - Fuller, F. L

$411,29,5$. Fuller, I. A

415. 4.35 Fuller, I. I

41,169 Fuller, L. K

442.953 , Fuller, $1 \mathrm{r}$. C

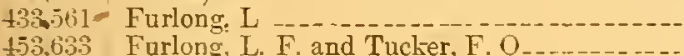

t31,1 11 Gale G. F

415,11 Grallagher, J. J

tti, 6.6 Gammon, G. $\mathrm{C}$

4.5,5,037 Gardner, F .

414,381 Gardiner, J. B

13.5,116 Gardiner, R. MI

142,30: Garland, W. H

430,755 Gartner, A

45:-63s Gärtner, A. F. and Harris, J. C

t5,, 01 Gürtner, A. F. and Harris, J. C

450,541 Gatley, E. J

437.68 .3 Gavnor, T. F

451,1:1 Gaynor, T. F

145,991 Gaynor, T. F

$4.51,666$ Gee, E. C

436,410 Geury, J

438,233 Geary, J

$438,23 \pm$ Geary, J
410,471 Gibbs,

t.jo,101 ribls, J. S

436,031 (tibbs, J. S. and Perkins, C. G

$4: 39,2+2)$

130,939

435,619

$442.61 \%$

435,1720

$t+2,11 \pm$

$414,=-2.5$

$435,43 \pm$

$41,04.5$

432,314

433.165

$4,39,3918$

$43: 2.5 \div 6$

$4+3,5,4$

$+39,941$

4.53 .000

$436,-(1) ;$

431,90

$431,7+2$

$434,8.2-1$

$436,7 \div$

$436, \pm 1 \cdot 2$

$47, \pm 2 t ;$

$4.31 \%$

$4.33,254$

448,90

$4,7,986$

432,197

$448, \cdots, 5)$

$4.1 .24 \%$

439,340

$43 \overline{0}$,

$4.2,+29$

445,024

4:0,068

441,8937

441,839

441,340

4. 4,430

453,695

$43,-7 \pm 11$

441,953

441,954
Gibson, C. D. P

Gilbert. E. F

Giles, J. E

Giles, J. E

rillette, II

Gillette, $\mathrm{H}$

Gillette, $\mathrm{H}$

Ginochio, N. B

Ginoehio, I. P

ripperich, A

Glover, J. B

Foldsehmidt.

Fioldstone, L

roodridge, IV. M

Goodson, G. A

Goodwin, C. $\mathbf{R}$

roodwin, C: R

Grorton, A

Goss, E. W

Gould, F. D'

Gould, C. C

Gould, R. H

Gould, IV. P

Graff; E. D

Gralıam, -

Graham, R

Graham, $G$

Granville, IF. P.

Graves, W. D

Grav, E.

(iray, W

Grefen, $\mathrm{H}$

Greenfield, E. T

Greenfield, E. T

rireenfield, E. T

Greenfield, E. T

Griscom, iV. W

Groswith, H

Groswith, HI
froswith, HI
Goddard, C-
Meter's

Motors

Cut-outs, meelıanieal

Galranometers

Magneto-eleetric

Carburetors, gas and air mixers -

Time chccks

IVater miges, flont $--=--$

Time eheeks

Musie, electric aetions

Medical and surgieal, body-wear

Insulators -...--...

Telegrapliy, fire

Alarms

Cut-outs, eleetro-magnetic

Drilling and boring

Electrodes

Lights, are, speeial features _..............

Conneetors

Regulators

Lanops and gas fittings, globes

Lights, are, speeial features

Insulators.

Bells

Annunciators

Time checks

Bells

Telegrapliy, relays and sounders

Alarms, burglar

Lighting systems, ear

Cut-outs, thermal

Cnt-outs, mechanieal

liatteries, seeondary

Filicostats -

Lighting; systems .........................

Lights, arc, gravity feed, gear-clutel _.....--

Railway gates, elcetric - do

-

Magneto-electric, hand waehines ..........

Telegraphy, tire

Clueks, electric

Indicators

Cut-outs, thermal

Lamp shades. -

Cordage, warp, stop motion

Indicators

Telephones, switeh-board:

Printing, matrix-making

Batteries, galranie

Railways, systems, elosed conduit

Eleetric loeomotires......

Telephones, systems.

Ligliting, systems

Shovels, tongs, and pokers Telcpliones, systems

Igniting deviees, manual

Insulators

Magneto-electric.

Ship's logs _..........

Lights, are, gravity feed, gear-clutch

Mlotors

Coin eontrolled apparatos, miseellaneons...

Magnetie loeks and bolts

Conduetors

Pheostats

Conductors, underground

Conneetors

Batteries, secondar

Motors

do
D.TE.

May 26,1891

May 26, 1891

July 15,1890

Oct. 21,1890

Mar. 17, 1891

Apr. 28, 1891

Sept. 2, 1890

Jan. 6, 1891

Dee. 23, 1890

Jan. i, 1891

Mar. 24, 1891

Aug. 5,1890

June 9, 1891

July 1,1890

Mar. 24, 1891

Mar. 2, 1891

June 30, 1891

Jan. 20, 1891

Aug. 26, 1890

Dec. 9,1890

Nov. 4, 1890

May 19,1891

May 19, 1891

Apr. 14, 1891

Oet. 7, 1890

Apr. 28, 1891

Feb. 10, 1891

May 5, 1891

Sept. 16, 1890

Oct. 14,1890

Oct. 14,1890

Mar. 31, 1891

Apr. 7,1891

Sept. 9, 1890

Oct. 28,1890

Nor, 1,1894

Oet. 21, 1890

Dec. 16,1890

Oet. 21, 1890

Dec. 9,1890

Jan. 20, 1891

Oet. 14,1890

Feb, 24, 1891

Oet. 14, 1890

July 29, 1890

Oct. 28,1890

July 22, 1890

Dec. 30, 1890

Nor. 4, 1890

May 26, 1891

Sept. 16, 1890

July \&, 1890

July 8, 1890

Aug. 19, 1890

Sept. 23, 1890

Sept. 16, 1890

Mar. 3, 1891

Apr. 28, 1891

July 29, 1890

Mar. 너, 1891

Oct. 7,1890

July 15,1890

Mar. 2-t, 1891

Apr. 28, 1891

Nor. 1,1890

Sept. 9, 1890

May 19, 1891

Mar, 10, 1891

Apr. 7, 1891

Dee. 2, 1890

Dec. ¿, 1890

Dee. 2, 1890

June 16, 1891

June 9,1891

Sept. 2, 1890

Dee. 2, 1890

Dec. 2,1890 


\begin{tabular}{|c|c|c|}
\hline No. & $\therefore$ NAME. & Cluss. \\
\hline $4 \pm 2,115$ & 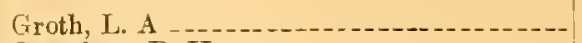 & Tanning processes \\
\hline 450,458 & Gruschow, R. H - & Annunciators, drop \\
\hline 578 & Guerrant, G. M ... & Engraving machines \\
\hline 344 & Guiley, A. H. R & Railway signals, cab, sectional rail_...---- \\
\hline 666 & n-10- & Lights, incandescent, special features . . . . \\
\hline 11 & Gunning, J. H _... & Rheostats _. \\
\hline 220 & aing, J. H & Electrodes - \\
\hline 60 & Gutmann, $\mathrm{L}$ & Systems of distribution \\
\hline 446,515 & ann, $\mathrm{L}$ & 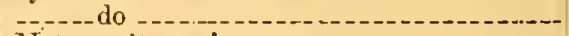 \\
\hline 410 & $\operatorname{ann}, L_{-}$ & Motors, alternating \\
\hline 447 & , H. J. & Lights, sockets and keys \\
\hline 45 & ock, W. E & Indicators \\
\hline 442,879 & Haight, H. J - & Thermometers \\
\hline 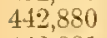 & Haight, H. J & Indicators \\
\hline $44^{\circ}$ & Haight, H. J & 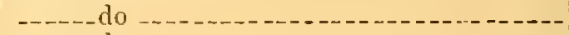 \\
\hline 442,882 & Haight, H. J & 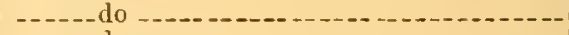 \\
\hline 442,883 & Haight, H. J & 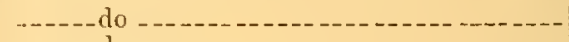 \\
\hline & Haight, H. J & - n-n-n \\
\hline
\end{tabular}

431,516 Haines, A. Z. and Curry, J. H 451,379

Cut-outs, mechanical

Hale, C. A

Insulators..-

Hale, G. C. and Barrett, A

414,700 Hall, 1 . I

453,046

$449,77 \%$

453,861

453.002

Hall, O. B

IIallbauer, A. L. and Hiller, E. L

Halpine, N. J

llamilton, J. E

Hammer, E. G

Hand, G. D. and Sells, O. P

Hannah,

Ilanson, E. II

IIanson, II

Harbeck, II, II

Harding, C. K

$440,5,0$ Hardy, F. D

$447,3: 1$ Hargrare, $T$.

477,449 IIargrave, T.

$453,4 i 6$

$441,4<7$

434,10

450,110

$446,2,2$

437,112

147,7,

$443,94)^{\circ}$

455,016

$4.46,917$

432,040

453,355

435,893
$+35,89$

435,89
$4+1, \pm 1$

433,983

$434,590^{\circ}$

454,598

436,354

441,066

445,176

437,901

435,203

$40,9 \div 0$

439,151

432.237

442.560

432,615

441.458

443,237

454,475

454,476

$435,69 \overline{0}$

IIarness, C. I' --

Farris,

Tarris, 1

Uarris, $\mathrm{T}$

Harrison, E.

Hart, G. W

Iarte, 11 . I

Harrey, E. T

Haskins, C:

IInswell, $\mathrm{A}$.

Hatch, A.

Hatch, G. E

Hatch, D. P. and Guion, P. M

Hathaway, $\mathrm{P}$

Hathaway, $\mathrm{P}$

Hayes, H. ${ }^{\top}$

Haves, J. $A$

Hazeltiue, C. I

Ieisler, $\mathrm{C}$

Heller, F. G. A

Hellesen, II. I. I

Henderson, II. B

Henning, B. F

Hensler, IV. S. and Grindley, IT. H .....

Heppell, R. II

Hering, $\mathrm{C}$

Herrick, A. B

Lighting, systems

Towers, telegraph poles.

Railways, conductor supports and insulators

Ships, sulomarine and torpedo.

Harps and hangers.

Clocks, electric

Tele phones, systems, toll

Cut-outs, mechanical

Sterentype plate holders

Batteries, galvanic

Photograplir, combined apparatus .........

Failways, sistems, clused conduit

-...-.do

Regulators

Printing, matrix-making

Electrudes

Magneto-electric

Tending apparatus

Coin controlled apparatus, cxhibitors, surface Regulators

Cial ramometers

Railway signals, block systems _..........

Cut-nuts, mechanical

Comelnctors, underground

Amunciators

Railway signals

IIotors

Cordage, warping

Electro-deposition

Telephones, calls do

Batterics, sccondary

Ore and coal washers

Telegraph5, circuit closers

Batteries, galvanic

Igniting rlevices, manual

Cut-nuts, thermal

Lichts, arc positice feed, rear-clutch--or--

Lights, arc, spccial featules _..........

Lights, sockets and kevs -

Lights, filaments and carbons.............

Balleries, galvanic -

Iilcetric locomotires

Lights, arc, grarity feed, gear-clutch _......

Ruilways, inclined planes

Prrometers

Painting machines -

Regulators

Galvanometers

Galvanometers

Cut-outs, mechanical
Date.

Dec. 9,1890

Apr. 14, 1891

July 8, 1890

June 16, 1891

Dec. 16,1890

Sept. 23, 1890

Nay 12, 1891

July 1, 1890

Feb. 17, 1891

Feb. 24, 1891

Mar. 3, 1891

June 9, 1891

Dec. 16,1890

Dec. 16,1890

Dec. 16,1890

Dec. 16,1890

Dec. 16,1890

June 30, 1891

July 1, 1890

Apr. 28, 1891

Nov. 25, 1890

Nov. 11, 1890

Jan. 13, 1891

May 26, 1891

Apr. 7, 1891

Aug. 5, 1890

June 9, 1891

May 26,1891

Tan. 13, 1891

May 26, 1891

Mar. 17, 1891

IIar. 31, 1891

Der: 23, 1890

May 12, 1891

Nov. 25, 1890

Sept. 30, 1890

IIay 5, 1891

Nov. 11, 1890

Mar. 3, 1891

Iar. 3, 1891

June 2, 1891

Yov. 25, 1890

Aug. 12, 1890

June 30, 1891

Feb. 10, 1891

Oct. 7, 1890

llar. 10, 1891

Mar. 3, 1891

Ja11. 6,1891

June 30, 1891

Felb. 24, 1891

June 2, 1891

July 15,1890

June 2, 1891

Sept. 2, 1890

Scpt. 2, 1890

Nov. 25, 1890

Ang. 1:2, 1890

Aug. 19, 1890

June 23, 1891

Sept. 16,1890

Jov. 18, 1890

Jan. 27, 1891

Oct. 7, 1890

Aug. 20, 1890

Mlar. 24, 1891

Oct. 28,1890

July 15, 1890

Dec. 9, 1890

July 22, 1890

พั้จ. 25, 1890

Dec. 23, 1890

Junc 23, 1891

June 23, 1891

Sept. 2, 1890

June 16, 1891

Aug. 26, 1890 
No.

NAME.

435,931 Herrick, C. H

$4+0, S 45$ Herrick, C. $\mathrm{H}$

454,650 Herzberg, I. and A -

t31,973 Herts, J. T

431,810

$435,+21$

451,870

443,632

443,633

453,863

$43 \pm, 411$

450,153

11,108

$43: 310$

437,116

450.160

445,798

435,660

437,359

437,360

447,826

447,827

453,538

442,048

445,751

445,752

432,61

432,619

435,629

44,067

451,691

$438,-36$

439,594

434,869

435,60 은

415,44

443,282

43,293

$4.5,5 ; 1$

411,304

436,235

441,502

441,55 ?

436,574

442,623

445,113

444,24 .

448,040

435,896

410,106

441,043

437,203

437,204

437, 20 .

437,206

431,519

431,720

431,97

432,049

432,136

$4: 3,692$

$432,-52$

432,75

483,170

$49.3,4117$

433,405

434,030

434.147

$4: 34,14$

$434,27.5$

434,276

$434,3 \times 5$

434,390

434,391

434,871

4.5, $66 \% 2$

435,879

Hirlimann, C. J
Hess, E. C

Hewett, E. M. G

Hewett, E. M. G

Hermann, E. and F. W'

Heynann, E. and F. W

Hibbard, $A$.

Hickley, A. S

Higham, D

Higham, D

Hinphey, J. J

Hines, $\dot{\mathrm{D}}$

Hobby, V. M

Hochhausen, II

Hochhausen, II

Hochhausen, II

Hoclibausen, II

Hodges, N. D. C

Hofiman, J. IV

Hoffman, J. W

Holcombe, $\mathrm{A}$. G

Holcombe, A.

Holcombe, A. G

Holcombe,

-

Hollar, W. H. and Holmes, F. S

Hollingshead, W. B

Hollingshead, IV. B

Hollingshead, W. B

Holmes, J. H

Holmes, H. E. and Grosrenor, C. F

Holmes, H. E. and Grosvenor, C. F

Holson, A. B

Holtzer, C. IV

Hoop, G. D.

Hepkinson, J

Hopkinson, $\mathbf{J}$

Huppes, J. J

Hopson, R. C

Hörenz, F

Hormel, A

Hornberger, W

Howell, J. IT

Hoyt, L. S

Hoy, A. H

Hunter, G. E. and Corthell, F. H

Hnnter, G. E. and Corthell, F. H

Hunter, G. E. and Corthell, F. H

Hunter, G. E. and Corthell, F. H

Hunter, R. M

Hunter, R. YI

Hnnter, R. II

Hunter, R. M

Hunter, P. M

Hunter, P. MI

Hunter, P. M

Hunter, P. II

Hunter, R. M

Hunter, R. M

Hunter, P. II

Hunter, P. MI

Hunter, R. MI

finnter, R. M

Hunter, P. M

Hunter, R. M

Hunter, R. J

IInnter, R. II

Hunter, R. MI

Honter, P. M

Hunter, R. M
Cliss.

DATE.

Lights, sockets and kcys

Cut-outs, mechanical

Telephones, supports pports and insulators- July 8,1890

Batteries, galvanic ...................... Sept. 2, 1890

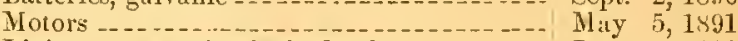

Liglits, arc, gravity feed, clutch _.......... Iec. 30, 1890

Dec. 30,1890

June 9,1891

Telephones, switch-boards

Batteries, secondary

Railways, system

Conductors --

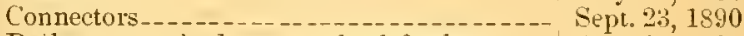

Railways, track cleaners, wheel fenders. _... Apr. 14, 1891

Batteries, galvanic _..._._... Mar. 24, 1891

Lights, tilaments and carbons _......... Fept. 2, 1890

Cut-outs, mechinical _..._._._._._...... Sept. 30,1590

Magneto-electric _............ Sejt. 30,1890

Cut-outs, electro-magnetic

Votors _._. Mar. 10, 1891

Lights, are, gravity feed, clutch _.......... June 2, 1891

Lightning rods _..._........ Dee. 16, 1890

Connecturs - Feb. 3, 1891

Cut-outs, mechanical _.................. Feb. 3, 1891

Telegraphy, fire ....................... July 22, 1890

July 22,1890

Jan. 6,1891

Railways, systcms, closed conduit _._...._._. May 5, 1891

Magnetic locks aud bolts_._._._._._._. Oct. 14, 1890

Batteries, secondary _.................... Oct. 28, 1890

A $119.19,1890$

Sejt. 16,1890

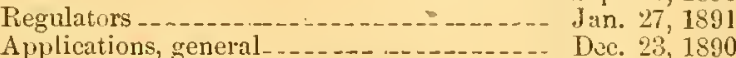

Applications, generations, tools and machines …..... Dec. 23, 1890

Applications, general _..._................ Nay 19, 1891

Annunciators _....._._._._._. Nov. 25, 1890

Telegraphy, lightning arlesters............ Sept. ?, 1890

Galvanometers _......... Jan. 13, 1891

Systems of distribution _..._-_..._-_...- Jan. 13, 1891

Railways, systems, overhead, trolleys ....... Sept. 23, 1890

Railways, systems, overhead _..._..._..... Dec. 16, 1590

Time checks ......................... Jan. 20, 1891

Annunciators _..._. Jan. 6, 1891

Magneto-electric ...................... Mar. 10, 1891

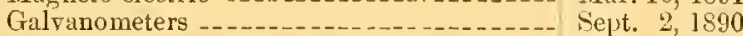

Railways, systems, overhead, trolleys _..._._. Nov. 4, 1890

Batteries, galvanic -..................... Dec. 2, 1890

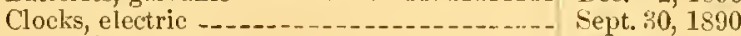

C..... do -

do -do

Railroads, systems, conduit $-0-5-1$

Electric locomotives................... July 8, 1890

Railways, systems, circnits, multiple arc ..._ July S, 1890

July 15,1890

Electric locomotives .................. July 15, 1890

Railways, systems, overhead, trolleys --

Electric locomotives

Railways, systems, overhead, trolleys

Electric locumotives

Railways, systems, conduit

Electric locomotives do

Railways, systems, circuits, multiple are

Railways, systems, overhead, trolleys. -

Railways, systems, conduit

Electric locomotives

Railways, systems, telpher

Railways, systems, conduit

July 22,1890

July 22, 1890

July 22, 1890

July 29,1890

July 29, 1890

July 29,1890

dug. 12,1890

Aug. 12, 1890

Aug. 12, 1890

Aug. 12,1590

Aug. 12, 1890

Aug. 12, 1890

Aug. 12,1890

Aug. 12, 1890

A ug. 19, 1890

Sept. 2, 1890

Sept. 2, 1890 
No.

NAME.

437,158

438,847

439,069

439,070

439,597

439,662

440,595

440,596

140,597

441,305

$+41,565$

443,451

443,452

443,661

443,677

444,397

444,566

145,14

$+15,409$

$445,6-1$

445,95 ?

$1 \pm 0,81$

446,83

446,831

$+4,28$

$+48,61$

$418,65:$

+4ts is

448,523

449,797

$4500^{-1}$

450,586

$451,1,1$,

151,15

$451.40 \%$

$451,6 ! 12$

450,920

$+54,437$

$43.7,340$

$+37,397$

$437,92-$

$145,1-12$

449,95

$45: 3,24:-$

$+39,707$

442,508

$+51,933$

412.25

434,509

435,4 넌

439.510

416,684

451,559

$+41,665$

453,320

434,063

45.019

45) 1.125

$+36,001$

448,041

$434,8 \%=$

$+12,267$

$431,9-$

$+53,190$

451,13

450,489

431,079

$\$ 33,097$

431,721

449,67 ?

458,4183

$434,186^{\circ}$

136,895

$\pm 33,65$

437,362

$+32,670$

$+132,944$

Hunter, R. II
Hunter, R. MI

Hunter, R. M

Hunter, R. M

Hunter, R. MI

Hunter, R. M

Hunter, R. MI

Hunter, F. MI

Hunter, R. II

Hunter, R. MI

Huuter, R. II

Hunter, R. MI

Hunter; R. M

Hunter, R. II

Hunter, R. MI

Hunter, R. II

Hunter, R. MI

Hunter, R. MI

Yunter, R. MI

Hunter, R. II

Hunter, R. II

Hunter, R. MI

Hunter, R. II

Hunter, R. M

Hunter, R. MI

Hunter, R. MI

Hunter, li. II

Hunter, Ii. II

Hunter, R. M

Hinter, R. II

Hunter, R. II

Hunter, H. MI

Ilunter, li. II

Hunter, R. II

Hunter, Ii. II

Hunter, R. MI

IIunter, R. II

Hubbell, C. .

Iludie, $\mathrm{R}$

IIugher, 1

Inglies, J. S

Ilumans, II

Humbert. II

Humwel, 1 .

Huntington. C: II

II untiugton,

Hussey, C. A

Hussey, C. A

IIver, II. E

Ide, A. L

Illingworth; J, and Baker, A. II

Ingralıam, A.

Irving, F. K. aud Hill, F. II

Iske, $\mathrm{A}$

lske, 1 . and $A$

lves, E. H

Jaekson, A. II

Jaekson, S.: D.

Jaekson, IV. E.

Jacobs, II. E

Jacobs, s. J

Jaeobs, S. J

Jacques, II. I

Jaeques, II. II

Jaeques, IV. E. and Emmott, G. R

Jeaucon, J. 1

Jenks, W. J

Jenks, Wr. J

Jennings, $\mathrm{B}$

Jenniugs, $\mathbf{B}$.
Class.

DAte.

Eleetrie locomotives

Railways, systems, overhead

Railway's, systems, eonduit

Oct. 21,1890

Railways, sytems, circuits, multiple arc _.... Oct. 21, 1890

Railwars, systems, conduit _............. Oct, 28, 1890

Railwars, svstems, overhead ............. Nov, 1, 1890

do _..... Nov. 11, 1890

Railwars, systens, eonduit_..._..._._. Nov. 11, 1890

-_._- do _- Nov. 11, 1890

Railways, svstems, battery -......... Nov. 25,1890

Eleetrie locomotives_._._._._._._._. Nor. 25, 1890

Railways, systems, conduit -............... Dee. 23, 1890

Railways, systems, orerhead, trolleys_._._.- Dec. 23, 1890

Car brakes _........... Dec. 30, 1890

Electrie loeomotives _............... Dec. 30, 1890

Railways, systems, overhead, trolleys ...... Jau. 6, 1891

Jan. 13, 1891

Jan. 20, 1891

Jan. 27, 1891

Feb. 3, 1891

Feb. 3, 1891

Feb. 17, 1891

Feb. 17, 1891

Feb. 17, 1891

Feb. 24, 1891

M Iar. 17, 1891

Mar. 24, 1891

Mar. 2-1, 1891

Mar. 17, 1891

Apr. 7,1891

Apr. 7,1891

Apr. 14, 1891

Apr. 28, 1891

Apr. 28, 1891

A pr. 28, 189

Nay 5, 1891

May 26, 1891

June 16, 1891

Aug. 26, 1890

Scpt. 30, 1890

Oet. 7, 1890

Batteries galranie

Telegraphy, lightning arresters

Telepliones, supports

Jan. 20,1891

Telephones, magnetie _...-.............- Mar. 31, 1891

Ingnetu-electric, hand machines ........ June 2, 1891

Molor - July 22, 1890

Telegraphy, circuits aud systems ...... Dec. 16, 1590

Connectors........................ Miky 12, 1891

Lights, sockets and kers _._._._......._._. Dee. 9, 1890

Cut-outs, thermal _.

Cut-onts, mechanical _..................... Sept. 2, 1890

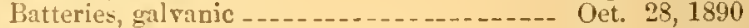

II

Motors _........ MLay 5, 1891

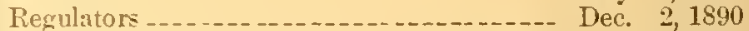

Railwas, eonductor supports and insulators _ June 2, 1891

Street cars, brakes

Railways, srstens, battery _. _............ June 30, 1891

Yeters

Batteries, galuanie -........... Sept. 9, 1890

Telegraphy, eireuit closers ............... Mar. 10, 1891

Fire alarms -..... Aug. 19, 1890

Telegraphy, multiplex-_-_- Dee. 9, 1890

Iedieal and surgicul, body-wear .......... July 8, 1890

Adrcrtising, antomatic devices _............ June 2, 1891

Railwar signals_............ Apr. 28, 1891

Fiailways, systems, overhead, trolleys_..... Apr. 14, 1891

Telegraphy, fire, alarms and extinguishers -- July \&, 1890

Railway signals _..._._._._._._._._. Aug. 26, 1890

Lights, incandescent, speeial features ......- July \&, 1890

Telephones, systems _................ tpr. T, 1891

-..._-do .............. June 2, 1891

Cut-outs, thermal

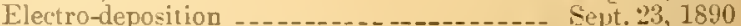

Telegraphy, lightning arresters......... Aug. 5, 1890

-

Tailways, svstems, eonduit ............... July 22,1890

July 20,1890 
No. NAME.

Cluass.

DATE.

451.326 Jennings, B

15.2,52: Jennings, $\mathrm{B}$

452,645 Jennings, B

$452,5 i \pm$ Jenner, C. D

142,053 Jewell, $R$

14,35s Jerell, $\mathrm{R}$ -

436,979 Jirnn, C. G

$411,16.5$ Johns, H. IT

$433,+71$ Johns, H. W

433,472 Johns, H. IV

433,473 Johns, H. IT

431, s11 Johnson, E. H

431,812 Johnson, E. H

443,526 Johnsou, E. H

443,606 Johnson, E. H

43.5, 897 Johnson, E. H

435,998 Johnson, E. H

435,899 Johnson, E. H
48,431 Johnson, E. H

445,431 Johnson, E. H
419,044 Johnson, E. H

450,- J.2 Johnson, E. H

450, it4 Johnson, E. H

451,270 Johnsou, E. H

435,098 Johnston, A. I

42,137

450,292

450,159

$451,13: 2$

436,857

433,082

$\$ 1,847$

$4+3,527$

$41,, 64$

442051

453,710

$452.186^{\circ}$

445,954

437,602

$44^{-}, \pm 09$

437,36 ?

432,050

440,164

440,165

433,409

437,859

$443, i-2$

$+53,260$

452,306

44.374

452,305

41,959

441,958

47,569

439,011

438,814

450,626

440,042

$4+5,146$

$45 \overline{5}, 237$

$+32,490$

$443,6+6$

43,097

431,062

$48,7.1$

452,926

452,79

$+47,832$

447,074

450,238

439,015

$44,4=4$

443,455

443,456

143,457

434,149

$452,501 \%$

Johnston, J. G

Jones, A. IV

Jones, B. J

Jones, F. R

Jones, F. IV

Jones, F. WV

Jones, F. W

Jones, $\mathrm{H}$

Jones, J

Jones, R. M

Jordan, L. F

Kaiser, $\mathrm{F} . \mathrm{P}$

Kalbach, H. M

Keeley, D. H

Keeley, D. H

Keithley, H. R

Kells, C. E., jr

Kells, C. E., jr

Keen, W. M. B

Kennedy, R

Kent, J

Kerstein, II

Kerstein, M

Kessler, IV

Keyes, B. P

Keyes, B. B

Khotinsky, A

Kim, G. II

Kinsman, F. E

Kintner, C. J

Kintner, C

Kintz, J

K̈irnan, IV. H

Kirnan, IV. H

Kirwan, $\mathrm{G} . \mathrm{H}$

Kitsee, I

Kitsee,

Kitiee, I

Klein, C. J
Johnston, W. C., jr

Kaiser, J. H. and Ledwith, A. B ........

Kammeyer, C. E

Kelles, D. and Parkhurst, M. C

Keen, IV. M. B. and Haarmann, IV. F

Keen, W. MI. B. and Haarmann, W. F

Kennedy, C. IV

Kennedy, C. IV. and Groswith, H

Kilbourn, W. H

Kimball, J. I. and Wirt, H.

KInmpp, J. F., jr
Railways, systems, conduit

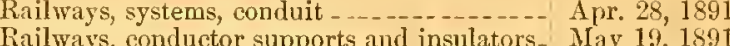

_- ._. do _...

Rheostats

Measuring vessels

Register's -......-

Measuring pumps

Boiler coverings -

Coverings

Gearing -

Regulators

Telephones, electric

Lighting, systems

Cut-outs, electro-magnetic

Valve-controllers

Regulators

Cut-outs, mechanical

Lighting, systems -

Electric locomotives.

Gearing

Conductors, underground

Cut-outs, meclianical

Batteries, secondary

Telegraphy, circuit closers

Cut-onts, thermal

Lighting, systems, car

Connectors

Telegraphy, circuits and systems do

Lighting, systems

Railways, systems, conduit

Railways, systems, overhead

Railways, systems, overhead, trolley

Lighting, systems

Telephones, details

Magnetic locks and bolts

Cut-onts, thermal

Inductoriums, transformers, stationary

Telegraphy, circuits and systems

Telegraphy, multiplex

Railways, systems, conduit

Telegraphy, dial

Applications, tools and machines

Dental instruments

Lights, sockets and keys

Lights, filaments and carbons

Lights, sockets and keys ....

Batteries, secondary

Batteries, galranic

Induction coils

Lights, arc, positive feed, gear-clutch

Lighting, systems

Insulators

Cut-outs, mechanical

Lights, sockets and keys

Telegraphy, printing

Applications, tools and machines

Drop lights and hangers

Cut-outs, thermal

Railway signals, sectional rail _.

Telegraphy, transmitter

Motors

Lights, incandescent, special fentures......

Telegraphy, file -

Station indicators

Batteries, secondary

- - ---do

-nds do

Lights, incandescent, special features -...-.

Telegraphy, fire, alarms and extinguishers -
Way 19,1891

May 19, 1891

Dec. 2, 1890

Jan. 6, 1891

June 2, 1891

Sept. 23, 1890

Nor. 25, 1890

Aug. 5, 1890

Aug. 5, 1890

Aug. 5, 1890

July 8,1890

July 8,1890

Dec. 30, 1890

Dec. 30,1890

Sept. 2, 1890

Sept. 2, 1890

Sept. 2, 1890

Mar. 17, 1891

Mar. 24, 1891

Apr. 21, 1891

Apr. 21, 1891

June 16, 1891

Aug. 26, 1890

Dec. 9, 1890

Apr. 14, 1891

A pr. 1f, 1891

May 5, 1891

Sept. 23,1890

July 29, 1890

Dee. 2, 1890

Dec. 30, 1890

Dec. 2, 1890

Dec. 16,1890

June 9, 1891

May 12, 1891

Feb. 3, 1891

Sept. 30,1890

Mar. 3, 1891

Sept. 30,1890

July 15, 1890

Nov. 11,1890

Nov. 11, 1890

July 29, 1890

Oct. 7,1890

Dec. 30, 1890

June 2, 1891

May 12,1891

Jan. 27, 1891

May 12, 1891

Dec. 2, 1890

Mar. 3, 1891

Oct. 21, 1890

Oct. 2], 1890

Apr. 21, 1891

Nov. 4,1890

Jan. 20, 1891

June 30, 1891

July 15,1890

Dec. 30,1890

Dec. 23,1890

July 1, 1890

Mar. 24, 1891

May 12, 1891

May 26,1891

IIar. 10, 1891

Feb. 24, 1891

Apr. 14, 1891

Oct. 21, 1890

Dec. 23,1890

Dec. 23,1890

Dec. 23,1890

Dec. 23, 1890

A 170. 12, 1890

May 19, 1891
Dec. 2, 1890 


\begin{tabular}{|c|c|c|c|}
\hline No. & NAME. & Class. & DATE. \\
\hline $\pm 47,833$ & Knight, C. M. and Hawken, T & Connectors... & Mar. 10,1891 \\
\hline 432,142 & Knight, W. $\mathrm{H}$ & Electric locomotives & July 15,1890 \\
\hline $\pm 47,332$ & Knight, W. H & Railways, systems, conduit & Mar. 3, 1891 \\
\hline 45$], 491$ & Fnight, W. $H$ & Electric locomotives & May 5,1891 \\
\hline 438,008 & Knudson, A. A & Conduetors & Oct. 7,1890 \\
\hline 439,959 & Kohrn, W. A. J. & Telcgraphy, keys - & Nor. 4,1890 \\
\hline 432,672 & Kookogey, W. P & Batteries, secondary & July 22,1890 \\
\hline $\pm 33,174$ & Kookogey, W. P . & - & July 29,1890 \\
\hline 434,133 & Kookogey, W. P & Heaters & Aug. 12, 1890 \\
\hline 432,053 & Korst, $\mathrm{P} . \mathrm{H}$ & Neters & July 15,1890 \\
\hline 452,796 & Fress, $\mathrm{G} . \mathrm{R}-$ & Lightning rods _...-.-- & May 26, 1891 \\
\hline 451,696 & Kuehnle, J _... - & Railways, systems, overhe & May 5,1891 \\
\hline 452,576 & $\begin{array}{l}\text { Tiuelninle, J J.... } \\
\text { Kunhardt D }\end{array}$ & Teleoraphy automatic_. & May 19,1891 \\
\hline$+37,307$ & $\begin{array}{l}\text { Kunhardt, } \mathrm{D} \\
\text { Kurmayer, } \mathrm{K}\end{array}$ & $\begin{array}{l}\text { Telegraphy, automatic_. } \\
\text { Lights, arc, positive feed....... }\end{array}$ & Sept. 30, 1890 \\
\hline $\begin{array}{l}448,351 \\
451,872\end{array}$ & $\begin{array}{l}\text { Kurmayer, K } \\
\text { La Boiteaux, F. M - }\end{array}$ & $\begin{array}{l}\text { Lights, are, positive feed } \\
\text { Motors }\end{array}$ & Mar. 17, 1891 \\
\hline $\begin{array}{l}451,872 \\
451,873\end{array}$ & $\begin{array}{l}\text { La Boiteaux, F. M } \\
\text { La Boitcaux, F. M }\end{array}$ & & May 5,1891 \\
\hline $\begin{array}{l}451,873 \\
449,288\end{array}$ & $\begin{array}{l}\text { La Boitcaux, F. M } \\
\text { Lalımeyer, WT- }\end{array}$ & $\begin{array}{l}\text { Pneumatic fans...-- } \\
\text { Regulators }\end{array}$ & May 5,1891 \\
\hline $\begin{array}{r}449,288 \\
437,8 \pm 8\end{array}$ & $\begin{array}{l}\text { Lalimeyer, W' } \\
\text { Lampert, H., jr }\end{array}$ & $\begin{array}{l}\text { Regulators } \\
\text { Batteries, secondary }\end{array}$ & Mar. 31, 1891 \\
\hline $\begin{array}{l}437,8 \pm 8 \\
437,654\end{array}$ & $\begin{array}{l}\text { Lampert, H., jr } \\
\text { Lander, G. and Simpson, J. H }\end{array}$ & $\begin{array}{l}\text { Batteries, secondary ---- } \\
\text { Heaters }\end{array}$ & Oct. 7,1890 \\
\hline $\begin{array}{l}437,654 \\
434,154\end{array}$ & & $\begin{array}{l}\text { Heaters } \\
\text { Galvanometers }\end{array}$ & Sept. 30,1890 \\
\hline $\begin{array}{l}434,154 \\
450,628\end{array}$ & $\begin{array}{l}\text { Lange, } \mathrm{P} \\
\text { Lange, } \mathrm{P}\end{array}$ & $\begin{array}{l}\text { Galvanometers } \\
\text { Lights, sockets and kers }\end{array}$ & Aug. 12,1890 \\
\hline $\begin{array}{l}450,628 \\
434,151\end{array}$ & $\begin{array}{l}\text { Lange, } \mathrm{P} \\
\text { Lange, } \mathrm{P} \text {. and Shallenberger, } \mathrm{O} \text {. B. }\end{array}$ & $\begin{array}{l}\text { Lights, sockets and keys }-.- \\
\text { Cut-outs, mechanical }\end{array}$ & Apr. 21, 1891 \\
\hline $\begin{array}{l}434,151 \\
434,152\end{array}$ & & Cut-outs, mechanical & Ang. 12,1890 \\
\hline 434,152 & $\begin{array}{l}\text { Lange, } P \text {. and Shallenberger, } O . B_{-} \\
\text {Linge, } P . \text { and Shallenberger, } O . B_{-}\end{array}$ & Lights, sockets and keys & Aug. 12,1890 \\
\hline $\begin{array}{l}434,153 \\
+39,017\end{array}$ & & $\begin{array}{l}\text { Lights, sockets and keys - } \\
\text { Telegraphy, reed }\end{array}$ & A ug. 12,1890 \\
\hline $\begin{array}{l}439,017 \\
453,261\end{array}$ & $\begin{array}{l}\text { Langdon-Daries, C } \\
\text { Langdon-Daries, C }\end{array}$ & $\begin{array}{l}\text { Telegraphy, reed - } \\
\text { - do }\end{array}$ & Oct. 21, 1890 \\
\hline $\begin{array}{l}453,261 \\
44,706\end{array}$ & $\begin{array}{l}\text { Langdon-Daries, C } \\
\text { Lane, T. W }\end{array}$ & Igniting devices, antomati & $\begin{array}{l}\text { June } 2,1891 \\
\text { Jan. } 13,1891\end{array}$ \\
\hline $\begin{array}{l}441,606 \\
444,896\end{array}$ & $\begin{array}{l}\text { Lane, T. W-- } \\
\text { lane, T. W W }\end{array}$ & --_- do - - - - & $\begin{array}{l}\text { Jan. } 13,1891 \\
\text { Jan. } 20,1891\end{array}$ \\
\hline 413,098 & Langstalfe, $\mathrm{J}_{-}$ & Incrustatio & Dec. 30,1890 \\
\hline 451,941 & $\begin{array}{l}\text { Lat Toint, T. T., Flanagan, J. II. and Thomp- } \\
\text { son, C. A. }\end{array}$ & Drain tiles_ & May 12,1891 \\
\hline 454,5202 & Larkin, G. II. and Tomkins, J & Ruilways, systems, overhead, trolley & June 23,1891 \\
\hline 997 & La lioche, F. A & $\mathrm{L}$ & June 2, 1891 \\
\hline 109 & Lattig, J. W-. & Railway sig & Oet. 28,1890 \\
\hline 416,527 & Laturent-C ly, A. M. F. and Timmis, I. A---- & Batteries, sec & Feb. 17, 1891 \\
\hline 44,780 & Lawrence, G. R- & Telephones, & Jan. 13,1891 \\
\hline 454,573 & Lawrence, W. H & Lights, filam & June 30,1891 \\
\hline$+31,698$ & Leaks, S. F - - - & Railways, pn & July 8,1890 \\
\hline 431,699 & Leaks, S. F ... & -...-do ... & July 8, 1890 \\
\hline 431,700 & Leaks, S. F - & -----do - & July \&, 1890 \\
\hline - & $\begin{array}{l}\text { leaks, } \therefore \text { F - } \\
\text { Lean, } \& \text { R - }\end{array}$ & 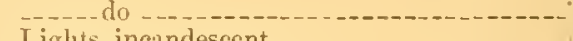 & July 8,1890 \\
\hline 431,776 & 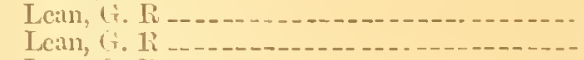 & $\begin{array}{l}\text { Lights, incin } \\
\text { Lights, socke }\end{array}$ & July 8, 1890 \\
\hline $\begin{array}{l}441,567 \\
4+5,957\end{array}$ & $\begin{array}{l}\text { Lean, (A. R } \\
\text { Lean, G. R } \ldots\end{array}$ & $\begin{array}{l}\text { Lights, socket } \\
\text { Cut-outs, mecl }\end{array}$ & $\begin{array}{l}\text { Jan. } 13,1891 \\
\text { Feb. 3, } 1891\end{array}$ \\
\hline $\begin{array}{l}4 \pm 3,40 \\
446,623\end{array}$ & Lean, G. R... & $\begin{array}{l}\text { Cut-outs, mechanical } \\
\text { - do }\end{array}$ & Feb. 17, 1891 \\
\hline $\pm 48,8 \pm 7$ & Leakin, J. II & Batlerics, galranic & Mar. 24, 1891 \\
\hline 419,206 & Lean, (i. R - & Lights, incar & M:ir. 31, 1891 \\
\hline 883 & Lebcnlorer, S - & Telephones, & July 22, 1890 \\
\hline 451,942 & $\begin{array}{l}\text { Lechuer, F. M } \\
\text { Lee, II. and Lebrun, E--- }\end{array}$ & Mining mac & May 1ะ, 1891 \\
\hline $4 \pm 7,13 \pm$ & $\begin{array}{l}\text { Lee, } \mathrm{II}_{\text {. }} \text { and Lebrun, E--- } \\
\text { Lee, } \mathrm{H}_{-}\end{array}$ & Printing, matrix-making & Feb. 24, 1891 \\
\hline $\begin{array}{l}7,135 \\
15,023\end{array}$ & $\begin{array}{l}\text { Lee, } \mathrm{H}- \\
\text { Lefebrre, } \mathrm{E}\end{array}$ & -----do do - & Feb. 24, 1891 \\
\hline 5,023 & $\begin{array}{l}\text { Lefebrre, E.-- } \\
\text { Lehman, J. H - }\end{array}$ & $\begin{array}{l}\text { Clocks, clectric -- } \\
\text { Igniting devices, }\end{array}$ & $\begin{array}{l}\text { Jan. } 20,1891 \\
\text { July } 22,1890\end{array}$ \\
\hline 44,707 & Lehman, J. II - & Kheostats ....... & $\begin{array}{l}\text { July } 22,1890 \\
\text { Jan. } 13,1891\end{array}$ \\
\hline 1,597 & Lehy, G. B -- & Alarms, burglar & July 8,1890 \\
\hline 1,598 & Lehy, (t. B - & Telegraplyy, & July 8,1890 \\
\hline 32,630 & Lenup, H -- & Heaters... & July 22,1890 \\
\hline 34,450 & $\begin{array}{l}\text { Lemp, If } \ldots \\
\text { Lemv, }{ }_{-} \ldots\end{array}$ & - do & Augr. 19,1890 \\
\hline $40,6 \pm 0$ & $\begin{array}{l}\text { Lemp, H } \ldots \\
\text { Lemp, II } \ldots\end{array}$ & $\begin{array}{l}- \text { do } \\
\text { do }\end{array}$ & Nov. 18,1890 \\
\hline $40,6+1$ & $\begin{array}{l}\text { Lemp, II } \ldots- \\
\text { Lemp, II } \ldots\end{array}$ & hts, filuments an & Now, 18, 1890 \\
\hline $\begin{array}{l}40,759 \\
43,224\end{array}$ & $\begin{array}{l}\text { Lemp, II } \ldots \\
\text { Lemp, II ... }\end{array}$ & hits, filam & Nov. 18,1890 \\
\hline 43,224 & $\begin{array}{l}\text { Lemp, II } \\
\text { Lemp, I. and Schr }\end{array}$ & $\begin{array}{l}\text { Heaters } \\
\text { Regulators, alterns }\end{array}$ & Dec. 23,1890 \\
\hline $\begin{array}{l}4,939 \\
16,97 t\end{array}$ & $\begin{array}{l}\text { Lemp, H. and Schmidt, L. Ml } \\
\text { Lemp, H. and Schmidt, L. MI }\end{array}$ & $\begin{array}{l}\text { Regulators, alterns } \\
\text { Heitters }\end{array}$ & Jan. 20,1891 \\
\hline $\begin{array}{l}4+6,974 \\
+326=9\end{array}$ & $\begin{array}{l}\text { Lemp, H. and Schmidt, L. M } \\
\text { Lcmp, H. and Tregoning, J }\end{array}$ & Heitters- -- & Feb. 24, 1891 \\
\hline $432.6 \div 9$ & $\begin{array}{l}\text { Lcmp, H. and Tregoning, J } \\
\text { Lemp, H. and Wightman, M. J.-. }\end{array}$ & Lights arc & July $2 \stackrel{2}{-}, 1890$ \\
\hline 54,485 & $\begin{array}{l}\text { Lemp, H. and } \\
\text { Lcnahan, F. P }\end{array}$ & $\begin{array}{l}\text { Lights, are, } \\
\text { Railway sis }\end{array}$ & June 23,1891 \\
\hline 4.625 & $\begin{array}{l}\text { Lcnahan, F. P } \\
\text { Lenhart, P. F- }\end{array}$ & $\begin{array}{l}\text { Railway signals, cab, sec } \\
\text { Netal founding wheels. }\end{array}$ & $J$ une 23,1591 \\
\hline $4 \pm 5,319$ & $\begin{array}{l}\text { Lenhart, P. F- } \\
\text { Leonard, B. W }\end{array}$ & $\begin{array}{l}\text { Netal founding, wheels.- } \\
\text { Telegraphy, fire, automati }\end{array}$ & Jan. 27,1891 \\
\hline $5-7 \pm 6$ & $\begin{array}{l}\text { Leonard, B. W } \\
\text { Leonard, H. W }\end{array}$ & $\begin{array}{l}\text { Telegraphy, fire, a } \\
\text { Regulators } . .---.\end{array}$ & June 23,1891 \\
\hline $435,-100$ & $\begin{array}{l}\text { Leonard, H. W- } \\
\text { Le Sueur, E. A-- }\end{array}$ & $\begin{array}{l}\text { Regulators - - } \\
\text { Electrolysis:- }\end{array}$ & Sept. 2,1890 \\
\hline 450,103 & $\begin{array}{l}\text { Le Sueur, E. A-- } \\
\text { Le Sueur, E. A-- }\end{array}$ & Electrolysis -. & Apr. 7, 1891 \\
\hline $50,10 \pm$ & $\begin{array}{l}\text { Le Sueur, E. A- } \\
\text { Le Sueur, E. A- }\end{array}$ & ---- do $_{-}$ & Apr. 7, 1891 \\
\hline 437,905 & $\begin{array}{l}\text { Le sueur, E. A } \\
\text { Leri, MI }\end{array}$ & Automatic derices & $\begin{array}{l}\text { Apr. } 7,1890 \\
\text { Oct. } 7,1890\end{array}$ \\
\hline $441,87 \pi$ & Lewis, F & Lights, incandescent, special features ....-- & Dee. 2,1890 \\
\hline 437,210 & Leber, L. H & Electric locomotires & Sept. 30,1890 \\
\hline
\end{tabular}


$4364: 5$
Libbey, H. W Libbey, H. IV

Libbey, H. W

Libbey, H. IY

Lichtenstadt, D. and C

Lieb, C. A

Lieb, C. A

Lieb, C. A

Lieb, C. A

Lieb, C. A

Lieb, C. A-

Lieb, C. A

Lieb, C. A

Lieb, C. A-

Lieb, C. A

Lieb, C. A-

Lieb, C. A

Lieb, C. A

Lieb, C. A

Lieb, C. $A$

Lieb, C. A

Lieb, C. A

Lieb, C. A. and Leavens, E

Lieb, C. A. and Learens, E

Liebig, G. A. and Willms, C

Linville, S. R. and Hettmansperger, L. F

Linrille, S. R

Linn, S. H

Litule, F. W. and Robb, J. T

Little, R. H

Little, G. an

Locke, F. M. and Lapp, J -

Locke, S. D

Lockirood, T. D

Loomis, O. P

Loomis, T. F

Loth, C. E

Lounsbury, J. A

Lor, I. WV, and O'Toole, $\mathrm{R}$

Luby, $\mathrm{Tr}$. A

Lucas, A. H

Ludlow, S. TV

Ludlow, S. W

Ludwig, J. C

Lundell, P - -

Luthe, J. W. and Jeavons, A. F

Luthe, J. W. and Jeavons, A. E

Lyke, $\mathrm{N}$

Lynch, J

Lynch, T. J

Lyon, J. B

Lyon, J. B

MacDonald, A. J

MacDonald, A. J

MacDonald, A. J

MacFarlane, J

Mackloskie, C. H. and Baker, W. E

Macmillan, W

Macrae, R

Macrae, $\mathrm{R}$ - -

Madden, $A$

Mallonee, J. D

Mallonee, J. D

Manaydier, J. E

Manning, $J$.

Mansfield, $F$

Mansfield, $\mathbf{F}$

Mansfield, $F$
Railways, systems, overliead

Railways, systems, conduit -

$$
\text { do }
$$

Electrodes

Connector

Wagneto-electric

Railways, conductor supports and insulator

Railways, systems, overhead, trolleys

Magneto-electric

Lights, sockets, and keys

Railways, systems, overhead, trolleys .

Connectors

Railways, conductor supports and insulators

Towers, telegraph poles

Railways, condnetor supports and insulators ---.--do -

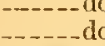

Towers, telegraph poles

Railways, conductor supports and insulators do

Masreto-electric.

Railways, conductor supports and insulators do -

Batteries, galvanic

Telegraphy, printing

$$
\text { do - }
$$

Lights, incandescent, special features

Electro-magnets - _.

Lights, incandescent, special features
Vending apparatus.

Motors -

Batteries, secondary

Insulators

Recolders

Telephones, electric

Cut-outs, mechanical

Railway signals

Conductors, underground

Lights, are, special features

Railway signals

Railway signals, sectional rail

Lights, arc, special features

Railways, systems, overhead

Telegraphy, fire, alarms and extinguishers Alarms

Telephones, relays and repeaters

Regulators _.

Railways, conductor supports and insulators

Annunciators

Medical and surgical, baths

Railways, systems, conduits

Drop lights and hangers

Telephones, switch-boards

Telephones, electric

Alloys -.-

Alarms

Railways, conductor supports and insulators

Batteries, galvanic

Railways, systems, overhead

Motors

Miscellaneous compositions

Magneto-electric

Printing, hand stamps

$$
\text { do - }
$$

Railways, systenis, telpher, solenoid

Lights, are, special features

Railways, systems, closed conduit

Locomotion

Electric locomotives.

Sept. 16, 1890

Sept. 2:, 1890

Nov. 25,1890

Nov. 25, 1890

Jan. 12,1891

Aug. 12, 1890

Aug. 12, 1890

Sept. 2, 1590

Sept. 2, 1890

Sept. 2, 1890

Tov. 18,1890

Nov. 25,1890

Dec. 30,1590

Jan. 13,1891

Feb. 24,1591

Mar. 17, 189

Mar. 31, 1891

A pr. 14, 1891

Apr. 14, 1891

Apr. 14, 1891

May 12, 1891

June $\stackrel{-}{-1} 1891$

June 23, 1891

June 23, 1891

June 23, 1891

Apr. 14, 1891

Apr: 14, 1891

Sept. 30, 1890

Apr. 21, 1891

Apr. 21, 1891

Oct. 21, 1890

May 12, 1891

Aug. 5, 1890

Aug. 12, 1890 Oct. 7, 1890 Apr. 28, 1891

Dec. 2, 1890

Dec. 9, 1890

Sept. 2, 1890

July I, 1890

กัธ. 18, 1890

Sept. 23,1890

Dec. 9,1890

Feb. 3, 1591

June 2, 1891

July 22, 1890

Mar. 10, 1891

Apr. 14, 1891

Apr. 14, 1891

Mar. 10, 1891

Oct. $\quad 7,1890$

Dec. 9, 1890

Sept. 2, 1890

Dee. 30,1890

Dec. 30,1590

July 15, 1890

Nov. 25, 1890

Apr. 7, 1891

Vay 26, 1891

Oct. 21, 1890

Oct. 21, 1890

Nor. 11. 1890

July 1, 1890

Feb. $2 \frac{1}{4}, 1891$

June 16, 1891

Apr. 28, 1891

June 2, 1891

Feb. 24, 1891

Mar. 3, 1891

Aug. 12, 1890 


\begin{tabular}{|c|c|c|c|}
\hline No. & & Class. & ATE. \\
\hline 32 & ansfield, F & Railways, conductor suppor & Dec. 9,1890 \\
\hline & $\mathrm{d}, \mathrm{F}$. & Railways, & Apr. 14,1891 \\
\hline & Jd, F & ....do - & Apr. 14,1891 \\
\hline & Mansfield, F. and & Telegraphy, lightning arre & June 30,1891 \\
\hline & S. W & ies, g & Aug. 5, 1890 \\
\hline & Marcher, $\mathrm{T}$ & & Oct. 14,1890 \\
\hline & Marcus, II. N.. & Telephones, details -.- - & Apr. 14, 1891 \\
\hline 59 & W. N - - & Tclephones, suppörts ...... & Apr. 14,1891 \\
\hline & H. W $W_{---}$ & Railways, sys & Oct. 21,1890 \\
\hline & $\mathrm{E}$ & 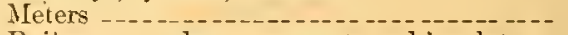 & Nov. 11,1890 \\
\hline & arks, W. B., Lau & $8 \mathrm{~s}, \mathrm{c}$ & Nov. 25,1890 \\
\hline & Marsh, J. W & tors _- & Apr. 14, 1891 \\
\hline & $T$ & & Dec. 9,1890 \\
\hline & & C & Dec. 9,1890 \\
\hline & G. W. & Lights, ine & Feb. 10, 1591 \\
\hline & & $\mathrm{T}$ & June 30,1891 \\
\hline & & hhy, & June 30, 1891 \\
\hline & ale, W. B & ng, ha & Apr. 7, 1891 \\
\hline & ale, W. B. & do & Mar. 24,1891 \\
\hline & $--1=--1$ & ies, se & Nor. 1], 1890 \\
\hline & H. N & $\mathrm{T}$ & July 1,1890 \\
\hline & & $\mathrm{B}$ & Oct. 28,1890 \\
\hline & 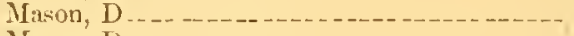 & $s, c o$ & Jan. 6,1891 \\
\hline & & 5 का & Jau. 6,1891 \\
\hline & B & tats & July 15,1890 \\
\hline & $\mathrm{H}_{-}$ & $\mathrm{Li}$ & Feb. 10, 1891 \\
\hline & H & & Feb. 10, 1891 \\
\hline & B & D & Oct. 21,1590 \\
\hline & A. & & May 26,1591 \\
\hline & A & & Apr. 21, 1891 \\
\hline & F. W & C & Mar. 31, 1891 \\
\hline & D... & B & Aug. 19,1890 \\
\hline & & M & Sept. 23,1590 \\
\hline & $\mathrm{C}$ & & 3,18 \\
\hline & $\mathrm{E}$ & $\mathrm{Li}$ & 6,1891 \\
\hline & R. & $\mathrm{T}$ & Mar. 10, 1891 \\
\hline & $L_{--}$ & $\mathbf{R}$ & Apr. 7, 1891 \\
\hline & & & Jan. 13,1891 \\
\hline & J. H & & July $\&, 1890$ \\
\hline 1 & & & Sept. 30,1890 \\
\hline & & & July 8,1890 \\
\hline & & & July 22,1890 \\
\hline & & & June 16,1891 \\
\hline & & & May 12,1891 \\
\hline & & & Oct. 21,1890 \\
\hline & & & Feb. 10, 1891 \\
\hline & $\therefore y$ & & July 1,1890 \\
\hline & & & Oct. $\quad 7,1890$ \\
\hline & & id & Sept. 30,1890 \\
\hline & 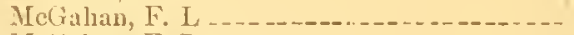 & -electr & Nov. +189 \\
\hline & 17 & Mot & June 30, 1891 \\
\hline & 18 & 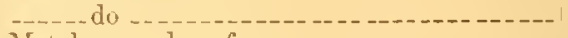 & 0,189 \\
\hline & & & Ma \\
\hline & $T$ & $c$ & Oct. 28,1890 \\
\hline & & & July 15,1 \\
\hline & & & July 15,1890 \\
\hline & & & July \\
\hline & & & July \\
\hline & & $\mathrm{C}$ & July 15, 1890 \\
\hline & & & July 15,189 \\
\hline & & & July 15,1 \\
\hline & & C & July 29,1890 \\
\hline & & & July 29,1 \\
\hline & 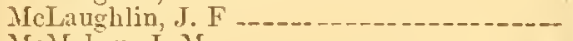 & & June 2,1891 \\
\hline & & & Oct. 14,1890 \\
\hline & & & \\
\hline & & & Nov, 18,1890 \\
\hline & & $\mathrm{Te}$ & Dec. 23,1890 \\
\hline & & & Mar. 3, 1891 \\
\hline & $\mathrm{F}-$ & Bat & Oct. 28,1890 \\
\hline & & Ap & Aug. 19, 1890 \\
\hline & $\mathrm{E}_{--}$ & In & Oct. 21,1890 \\
\hline & E. J. & $\mathrm{Te}$ & Feb. 24, 1891 \\
\hline & & Tel & June 16,1891 \\
\hline & 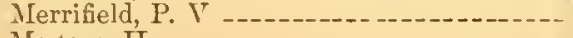 & & Dec. 9,1890 \\
\hline & & es, thern & Aug. 19,1890 \\
\hline & & & Dec. $16,18:$ \\
\hline
\end{tabular}


No.

Name.

$\pm 49,375$

44,734

452,276

$415,9 \times 0$

$4+7,195$

454,17

454,178

432,063

t:1,

451,83

453.982

137,930

443,195

44:2,071

$43 x .934$

$49,2-26$

449,490

452.611

452,546

453,553

449,610

434,024

433,52

$4.5,500$

442,650

436,717

437,501

42,316

432,934

439,417

430,416

436,371

$419,2 ?$

437,445

437,50

46,459

44.452

444,453

414,454

433,903

440,717

40,718

$41 ., 250$

450,970

433,904

43,157

434,992

435,951

$448,7+1$

452,758

$+49,750$

$45.5,10$

$45 \overline{5}, 2333$

$45.5,284$

438,586

437,613

449,989

447,896

$44 \overline{4}, 897$

$4.54,181$

449,249

442,385

449,690

$436,14,8$

438,779

$43 \overline{7}, \mathbf{7} 89$

448,359

$447,25 t^{\circ}$

433,251

445,475

443,536

449,168

$43.5,152$

450.973

$440,36,2$

439,022

$433,43.5$

Meyer, J. and Binder, C

Hiller, H. IT

Míller, I. N

Miller, J. E. A

Miller, J. J

Miller, J. J. C

Miller, J. D.

Milliken, G. F

Miner, IT: II

Mingle, G. W

Mino, J

Miro. F

Mitchell, A. W

Mitchell, A. II

Mitchell, E. IV

Mitchell, J. M

Mitchell, W

Ioffatt, R. R

Uoore, C. IV

Moore, G. R

Ioore, J. B

Morder, W. II

Morgan, R. A., jr

Morris, H. G

Morrison, $\mathrm{WT}^{*}$

Iorse, S. F. B

Morton, T. M

Mosher, J. I

Mosher, J. A

Iott, S. D

Mott, S. D

Mott, S. D

Mower, S. E

Mower, S. E

Hower, S. E

Mower, S. E

Mower, S. E

Mnehlberg, C. O

Muirhead, $\mathrm{A}$

Mueller, E. T.

Vüller, A. C. F

Munsie, J. F

Mnnsie, J. F

Iunsie, J. F

Mrphy, R. E

Narjot, E.

Nash, F. J

Nelson, R. E

Neu, G. S

Nerrman, A

Newton, E. C

Newton, S. O. and MI. A

Nichols, A. E

Nichols, H. P

Niles, H. P

Noriega, E

Norman, T. IV

Forman, T. W

Norton, J. A

Norton, $J$, A

Nourse, J. K. P

Noyes, B. J

Noyes, B. J
Meyrowitz, E. B. and Buchhop, F

Monrath, G. and Gihbons, IT. M .

Iorrison, W. and Schmidt, I.

Morse, P. C. and Winton, H. D

Mower, S. E and Spencer, G. J

Noyer, J. IT. and Jackson, G. F

Mïller, F. and Greene, J. G

Murray, J. WV

Tash E I Stont, G. G and Davis, J. R

Nickerson, W. E. and Berrenberg, A

Class.

DATE.

Telegraph poles

Motors

Meteris

Cut-outs, thermal

Annunciators

Railways, systems, conduit

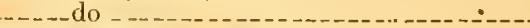

Bailways, systems, overhead, trolleys -

Applications, general

Towers, telegraph poles

Telegraphy, fire.

Telegraphy, multiplex

Cut-ourts, electro-magnetic--

Lamps and gas fittings, guards

Cut-outs, mechanical

Ruilways systems, orerhead trolleys-

Railways, systems, closed conduit

Iagneto-electric

Cut-outs, mechanical

Separators

Electrodes

Indicators

Figure wheeled toys

Lights, incandescent, special features

Telephones, supports _..............

Magneto-electric

Cut-outs, mechanical

Batteries, secondary

Regulators

Batteries, secondary

Railway signals

Railways, systems, circuits, multiple are

Conductors, underground

Lights, arc, positive feed, gear-clutch

Lights, arc, gravity feed, clutch

Indicators

- - - - do

Electric locomotives

-10
$-\cdots$
-

Store service, switches

Insulators.-.--..-

Lamp shades

Telephones, electric

Lighting, systems

Railways, conductor supports and insulators

Railways, systems, overhead

Batteries, galvanic

Railways, systems, overhead

Air and gas engines, explosive

Conductors.

Connectors

Cash registers

Telegraphy, fire, alarms and extinouishers

Annunciator

Magneto-electric, couplings

Drop lights and hangers

Lights, arc, special features

Conductors

Lights, incandescent

Glass, plate and window

Telephones, electric .

Wire

Conductors -.---.--

Cut-outs, mechanical

Railways, systems, hattery

Cut-outs, thermal

Telegraphy, fire

Mar. 31, 1891

Mar. 3, 1891

May 12, 1891

Mar. 24, 1891

Feb, 24, 1891

June 16, 189

June 16,1591

July 15,1890

July 8, 1890

May 5, 1891

June 9, 189

Oct. 7, 1890

Dec. 23,1890

Dec. ㄴ, 1890

Oct. 21,1890

Mar. 31, 1891

Mar. 31, 1891

May 19, 1891

May 19,1891

June 9, 1891

Mar. 31, 1891

Ang. 12, 1890

Aug. 5, 1890

June 23, 1891

Dec. 16,1890

Sept. 16,1890

Sept. 30,1890

Dee. 16,1890

July 22, I 890

Oct. 28. 1890

Oct. 28, 1890

Sept. 16, 1890

Mar. 31, 1891

Sept. 30, 1890

Sept. 30,1890

Feb. 17, 1891

Jan. 13, 1891

Jan. 13, 1891

Jan. 13, 1891

Aug. 5, 1590

Tov. 18,1890

Nov. 18, 1890

Feb. 24, 1891

Apr. 21, 1891

Aug. 5, 1890

Mar. 10, 1891

Ang. 26, 1890

Sept. 2, 1890

Mar. 24, 1891

May 19, 1891

Apr. T, 1891

June 30, 1s91

June 30, 1891

June 30,1891

Oct. 14, 1890

Sept. 30,1890

Mar. 24, 1891

Mar. 10, 1891

Mar. 10, 1891

June 16, 1891

Mar. 31, 1891

Dec. 9,1890

Apr. 7, 1891

Sept. 9, 1890

Oct. 21, 1890

Oct. 7,1890

Mar. 17, 1891

Feb. 24, 1891 
433,486 447,058 447,059 447,060 447,061 447,062 451,512 450,182 436,901 440,604 448,666 434,879 451,950 442,996

454,016 431,280 433,210 434,580 434,881
Noyes, B. J

Noyes, B. J

Noyes, B. J

Noyes, B. J

Noyes, B. J

Noyes, B. J

Noyes, B. J

Toyes, B. J. and Eaton, H. F

Nutting, S. E

Nutting, S. E

Nutting, S. E

Oakman, $\mathrm{s}$

Oakman, $\mathrm{S}$

O'Brien, J

$O^{\prime}$ C'mnell, J. J

Odell, J. B

Odell, J. B

Odell, J. B

Odell, J. B

Odell, J. B

O'Donnell, M. A

Oelirle, W. E

Oeyan, $\mathrm{T}$

O'Ileara, J

O'Meara, J

'Meara, J

O'Meara,

O'Neil, J

O'Neil, J. C

Ongley, C. E

Ongley, C. E

Ongley, C. E

Ongley, C. E

Ongley, C. E-

Ongley, C. E

Ongley, C. E

Ongley, C. F

Orcutt, E. L

Orford, J. M

Orford, J. M. and English, J. C

Otis, X. P

Otis, N.P. and Smith, R. C............... Ott, J. F

wen, A

Owen, R. O

Packer, W.

Paiste, H. T

Palmer, C. MI

Palmer, C. M

Palmer, D. A

Palmros, A

Parcelle, A. I

Parcelle, A. I

Park, H. s

Park, H. S

Parker, E. H

Parker, T. and Robinson, A. E

Parmly, S. P

Parrish, M. IV

Parrish, T. J

Parshall, H. F

Parsley, W. L

Parsons, B. J

Patterson, J. WT

Patterson, IV. R

Patterson, IV. $\mathrm{K}$

Patten, F. J

Patten, F. J

Pattison, R. and Desmond, D. G

Patton, IT. H

Paul, W. C. and Kleinstercher, A. D....

Pauthonier, $\mathrm{C}$
Telegraphy, fire

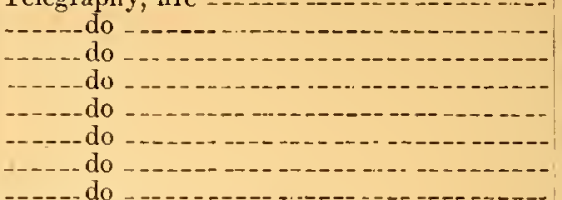

Regulators

Lights, are, positive feed...................

Magneto-electric

Insulators

$$
\text { do }
$$

Telephones, switch-boards

Telephones, systems

Perforating lace paper

Electric locomotives do

Telegraphy, tire

Telephones, electric

Talve controllers

Heaters

Rlieostats

Heaters

Comp's logs

Railway signals

Telegraphy, fire, alarms and extinguishers.

Telegraphy, fire -....

Lighting, systems ..

-

Valve controllers

Recorders

Connectors

Lights, sockets and keys

Cut-outs, mechanical

Elevators

Applications, tools and machines -......

Electric locomotives

Iedical and surical body-trear

Railway signals

Clocks, electric

Lights, incandescent, special features

Applications, tools and machines -...-

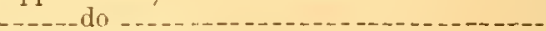

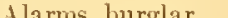

Rialways, systems, orerhead, trolleys .....- Apr. 14, 1891

Electric locomotives_.__..... July 29, 1890

- - - do no nuly 29, 1890

Magneto-electric _..._......._._._._._. Apr. 21, 1891

Time checks _._._.

Electro-magnets _........................ Apr. 28, 1891

Dampers, automatic_..._................ June 30, 1891

Telegraphy, reed _..................... Feb. 24, 1891

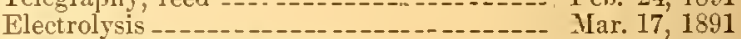

Lights, arc, special features ............ Aug. 5, 1890

Batteries, galvanic ........................ July 1, 1890

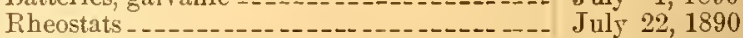

Magneto-electric _..._._._._._._._._._._._. Feb. 17, 1891

Conductors, underground _............... July 8, 1890

Railways, systems, overhead, trolleys.----- Mar. 31, 1891

Tending apparatus -

Conductors, underground -............... July 29, 1890 Bells _............ Jan. 13, 1891

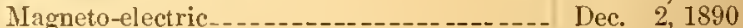

Motors, alternating …......... Feb. 3, 1891

-_-_-do -

Lights, incandescent, special features _..._-_ June 16, 1891

Electric locomotives_._._...............-. Aug. 26, 1890

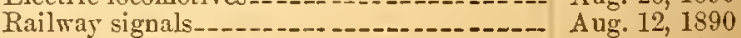

Lights, incandescent . .................. Oct. 28, 1890 
ร.

NAME.

Class.

D.TE.

440,268

440,269

$+40,270$

440,271

140,270

440,273

$440,2-4$

$+40,275$

410,276

$\pm 40,27$

40,575

414,223

451,514

445,927

434,835

440,189

434,45

434,45

41,80 -

436,057

436,107

439,150

435,47

432,169

48,669

435,516

434.032

438,359

439,18 ?

40,720

439,304

434,855

454,226

$\$ 40,015$

410,115

$\pm 54,949$

453.416

438,361

$44=, 838$

450,159

432.710

451, 100

43 ! 617

435,518

431,618

451,951

438,767

432,896

411,892

411,593

441,894

434,949

435,102

445,253

436,316

443,404

414,317

437,348

443,149

443,167

450,219

443,226

445,365

452,955

452,956

435,545

453,733

$440,-76$

434,199

442,390

442,391

437,510

430,215

437,512

$437,51 \%$

$43-, 661$

437,6692
Payen, C

Pasen, $\mathrm{C}$

Payen, C

Paren, $\mathrm{C}$

Payen, C

Payen, $\mathrm{C}$

Payen, C

Payen, C-

Paven, C

Parne, C. Q

Payne, J. I

Pearson, $\mathrm{P}$

Peckham, E

Pepper, D., jr

Pepper, D., jr

Pepper, D., jr

Perkins, C. G

Perkins, C. G

Perkins, F. B

Perret, F. A

Perret, F. A

Perls, T

Pfinnkuche, $\mathrm{G}$

Pfingst, $\mathrm{L}$

Pfingst, $\mathrm{L}$

Pfinger, C. A

Phillips, W. A.

Pieper, O. H. and Tratson, F. M

Pierce, $\mathrm{F}$

Pierce, F

Pilkington, H. II. and White, $\mathrm{h}$. S

Pinolet, L. M

Plumb, $\mathrm{C}$

Pocock, F. A

Poland, L. N. P

Poland, L. N. P

Pollak, C. F

Pollok, J

Polson, G. A.

Pope, E

Porter, C. S

Possons, TT. J

Possons, TI. J

Possons, W. .J

Possons, N. S

Possons, $\mathrm{Y}$.

Potter, J. S----ctur

Potter, J. S., Cartwright, D. J. and Keyes, B. BPotter, J. S. and Cartwright, D. J

Potter, J. S. and Cartwright, D. J

Powell, S. L

Powell, S. I

Powell, S. I

Pragnell, G. E

Pratt, J. I

Pratt, R. D

Prentiss, H. S

Prentiss, H. S

Prescott, G. B., jr.

Price, J. E

Pullman, H. B

Pnmpelly, J. K

Pumpelly, J. K

Pumpelly, J. K

Purdy, A. F

Rabinowicz, I

Rae, F:P

Pue, F. I

Rae, F. P

Rae, F. B
Lead

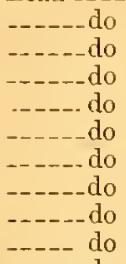

Separators

Car brakes

Electric locomotives

Telegraphy, circuits and systems

Electric locomotives

Batteries, secondary

Regulators

Cut-outs, mechanical

do --

Applications, tools and machines

Railwars, srstems, closed conduit

Magneto-electric do

Railway signals

Galranometers

Railways, systems, overhead, trolleys dlo

Cut-outs, mechanical

Lights, sockets and keys

Telegraphy, circuit closer

Alarms, burglar

Meters.

Annunciators

Telegraphy, fir

Railways, systems, conduit

Railways, grippers, whelp wheel

Lights, filaments and carbons..

Magneto-electric

Hardware making, miscellaneous

Incrustation

Regulators

Telegraphy, printing -

Clocks, pneumatic

Electro-magnets

Electro-deposition

$$
\text { do }
$$

Electric locomotive

Cut-outs, thermal

Lights, incandescent, special features

Lights, sockets and keys do

Insulators

Railway signals

-..-c do

Bells

$$
\text { do }
$$

Medical and surgical, body-wear

Lights, sockets and keys

Clocks, electric

Regulators -

Conductors, underground

Motors

Batteries, secondary

-...--do

Telegraphy, keys

Insulating materials

Telegraphy, dynamo

Cut-outs, mechanical

Electric locomotives

Motors

Tor. 11, 1890

Tov. 11, 18.90

Nov. 11, 1890

Xuv. 11, 1890

Nor. 11, 1890

Nor. 11, 1890

Nor. 11, 1890

Nov. 11,1890

Nov. 11, 1890

Nor. 11, 1890

Nor. 11, 1890

Jan. 6, 1891

May 5, 1891

Mar. 2t, 1891

tug. 19,1890

Nor. 11, 1890

Aug. 19, 1890

Aug. 19, 1890

Dec. 2, 1590

Sept. 9,1890

Sept. 9, 1890

Oct. 28, 1890

Sept. 2, 1890

July 15, 1890

Mar. 24, 1891

Sept. 2, 1890

Aug. 12, 1890

Oct. $1 \frac{1}{2}, 1890$

Oct. 28, 1890

Nov. 18, 1890

Oct. 28,1890

A ug. 19, 1890

June 16, 1891

Nor. 4,1890

Nov. 4,1890

June 30,1891

June 2, 1891

Oct. 14,1890

Mar. 2t, 1891

Apr. 14, 1891

July 22, 1890

Nay 5, 1891

July \&, 1890

Sept. 2, 1890

July \&, 1890

May 12, 1891

Oct. 2I, 1890

July 22, 1890

Dec. 2, 1890

Dec. 2, 1890

Dec. 2, 1890

Aug. 26, 1890

Aug. 26, 1590

Jan. 27, 1891

Sept. 9, 1890

Dec. 23,1890

Jan. 6,1891

Sept. 30,1890

Dec. 23,1890

Dec. 23,1890

Apr. 14, 1891

Dec. 23,1890

Mar. 17, 1891

May 26, 1891

Мат 26, 1891

Sept. 2, 1890

June 9, 1891 


No. $\quad$ Nane. $\quad$ Class. $\quad$ Date.

437,663 Rae, F. F

440,817 Rae, F. B

443,111 Rae, F. B

446,613 Rae, F. B

454,496 Rae, F. B

454,626 Rae, F. B

452,983 Radford, C. MI

455,055 Ramsiy, IV

440,502 Ranisey, J., jr., and Weir, F. C

440,503 Ramsey, J., jr., and Weir, F. C

443,004 Ramsey, IV. M

442,005 Ramsey, W. M

444,110 Randall, B. L

437,798 Ransom, G. F

441,972 Rapieff, $\mathrm{J}$

441,973 Rapieft; $J$

441,974 Rapieff, J

434,951 Raughtigan, $\mathrm{M}$

437, Ratt Rawson, F. I

45t,155 Readman, IV

450,872 Reardon, C. B

432,561 Reckenzaun, $\mathrm{A}$

437,763 Reckenzauu, $A$

$\begin{array}{ll}432,215 & \text { Redding, H- } \\ 448,587 & \text { Redfield, C. }\end{array}$

$\begin{array}{ll}448,587 & \text { Redfield, C. L } \\ 448,994 & \text { Redfield, C. L }\end{array}$

437,720 Reed, E. M.

441,256 Reese, T., jr

448,369 Regan, D.S

445,234 Reilly, J. C -

447,350 Reilly, J. C

450, 425 Reinemann, A

$\begin{array}{ll}434,159 & \text { Rcinmann, A. L } \\ 448,164 & \text { Reimmann, C. F }\end{array}$

440,505 Renault, B. and Desvernay, M ...............

433,216 Reppy, A. W

$444,10 \pm$ Reppy, A. WV

$\begin{array}{ll}438,527 & \text { Reynier, E. } \\ 146,61 \pm & \text { Ricc, D. II }\end{array}$

$\begin{array}{ll} \pm 6,61 \pm & \text { Ricc, D. H } \\ \pm 46,712 & \text { Rice, D. H }\end{array}$

$432,64 \pm$ Rice, E. IW.

440,654 Rice, E. W., jr

442,668 Rice, E. W., j r

$\begin{array}{ll}444,922 & \text { Rice, E. W., jr } \\ 447,352 & \text { Rice, E. W., jr }\end{array}$

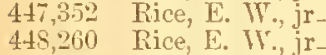

451,520 Rice, E. IV., jr

451,521 Rice, E. W
451,574 Rice, L. C

438,897 Richards, F. H

432,6 45 Richards, J. E

439,828 Richards, W. I

440,326 Richards, IV.

438,326 Richardson, G. IT

439,428 - Richter, C

451,226 Ricketts, J. I

431,482 Ries, E. E.

444,855 Ries, E. E

452,041 Ries, E. E

452,042 Ries, E. E

453,162 Ries, E. E

453,163 Ries, E. E

$453,16 \pm$ Ries, E. E

440,925 Riggs, J. W

440,926 Riggs, J. IV

440,927 Riggs, J. IV

441,703 Riggs, J. IT

443,227 Riker, A. L

452,717 Riker, A. L

452,718 Riker, A. L

437,868 Ritchie, A. M

$441, \$ 00$ Ritfield, E. H

40๊5,368 Rubb, J. T

434,468

433,421

449,174

Robbins, A. C
Robbins, H. G
Nagneto-electric

Telegraphy, printing

Railways, systems, battery

Telegraphy, lightning arresters.

Motors

Lightning rods

Railway signals

Railido -.....- Nov. 11, 1890

Railways, systems, overhead, trolleys---_--- Dec. 16, 1890

Railways, conductor supports and insulators Dec. 16, 1890

Electric locomotives _._._._._._._._. Jan. 6, 1891

Annunciators -....... Oct. 7, 1890

Distance instrument's ...................... Dec. 2, 1890

D_._do _._. Dec. 2, 1890

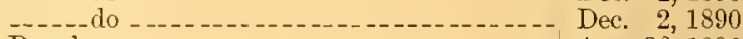

Regulaturs ........ Aug. 26, 1890

Insulating materials .................... Sept. 23, 1890

Condnetors, underground _........ June 16, 1891

Applications, general _._._._._._._._._. Apr. 21, 1891

Molors ...

Neters -

Igniting devices, manual_._._._._._._. _... July 15, 1890

Printing, matrix-making _..._._._._._. Mar. 17, 1891 do

Railways, systems, conduit

Magneto-electric _._._._._. . _._._._._. Nov. 25, 1890

Air and gas engines, explosive_....... Mar. 17, 1891

Conductors, anti-inductive _.___._. Jan. 27, 1891

Conductors, underground _.............. 3ar. 1891

Tclegraphy, circuit closers, thermal _...... Apr. 14, 1891

Lights, incandescent _._._._._._._._._. Aug. 12, 1890

Lights, incandescent, special features _-_.- Mar. 10, 1891

Batteries, secondary_................... Nov. 11, 1890

Telegraphy, railway-car -_._._-_._-_._-_ July 29, 1890

Lights, are, gravity feed, gear-elutch -...-- Jan. 6, 1891

Batterics, secondury _._._._._._._._._... Oct. 21, 1890

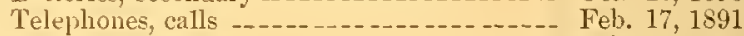

Electric locomotives _...-. July 22, 1890

Telegraphy, lightning arresters_......... Nov. 18, 1890

Regulaturs _._. Dce. 16, 1890

Electric locomotives____________._._. Jan. 20, 1891

Magneto-electric _..._._._._._._........... Mar. 3, 1891

Electric locomotives

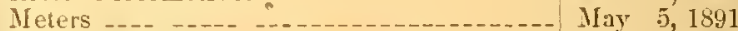

Telcgraphy, lightning arresters _..._.... May 5, 1891

Magneto-electric_........................ May 5, 1891

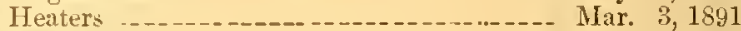

Separators -

Recorders

Telcphones, magnetic _................... Oct. 21, 1890

Ligliting, systems _............ Nor. 11, 1890

Oet. 14, 1890

Railways, systems, circuits, series -...-...-- Oct. 28, 1890

Annunciaturs, drop -................... Apr. 28, 1891

Railways, svstems, converter .............. July 1, 1890

Heater

Car brakes _........ May 12, 1891

Heaters.

Tempering and annealing-............. May 26, 1891

_._. do - n... May 26, 1891

Heaters -... May 26, 1891

Railway signals ................ Tov. 18, 1890

do

_.

Dec. 2, 1890

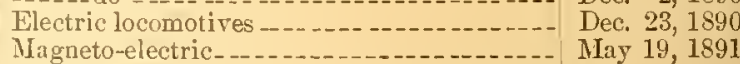

Magneto-electric_-_. May 19, 1891

Galvanometers ....................... Oct. 1890

Railways, conductor supports and insulators Dec. 2, 1890

Lights, incandescent, special features_..-_-- Aug. 26, 1890

Heaters ...................................... 19, 1890

Telegraphy, fire ......................... July 29, 1890

Telegraphy, transmitters 
$11: 2 ; 03$
Roberts, I. L

Roberts, I. L

Roberts, I. L

Folierts, I. L

Roberts, I. L

Roberts, I. L

Roberts, I. I

Ruberts, I. I

Roberts, II. J

Robinson, A

Rubinson, C. At

Robinson, F. and Lander, P. W. J

Robinson, M. IV

Robinsın, IV

Robinson, IV

Robinson, W

Robinson, II

Robinson, IF.

Roebuck, T. G

Roney, A. B

Root, H.

Rosebrigh, A. $\mathrm{I}$

Rosebrugb, A. MI

Rosenfield, W. II

Rothlisberger, E

Roulez, J. E

Röver, A. B

Rowland, H. A. and Duncan, I

Rowell, B. C. and Galloupe, F. E -....

Ruddick, J. J.

Rushmore, S. it

Rushmore, S. IV

Russell, E. C

Russell, E. C

Rutter, G. E

Sabold, F. W

Salathé, F

Salathé, F

Salisbury, IT.

Sargent, $F$

Sargent, $\mathrm{M}, \mathrm{O}$

Saunderson, $\mathrm{L}$

Sautter, $G$

Savage, IV. IT

Saw yer, R. F

Sawver, R. F

Scales, IT. S

Scales, W. S

Schatz, A. E

Schef hauer, $\mathrm{P}$

Scheuritzel, C. P. and Hess, J. L

Schiffmann, IV. IV

Schirner, 1

Sehlegel, H. T

Schleschinger, W. M

Schlesinger, W. II

Schmid, A

Schmid, $A$

Schmid, F. M

Schnidt, F. M

Schneider, J. F and Carson, D. K

Schoop, P

Schoop, P

Schreufer, J. G

Schreuder, J. G

Schroeder, G. G

Schroen, F. C

Schnltz, G

Schwarze, C. J

Schwarze, C. J

Schwarze, C. J

Schwartz, F

Scott, G. A
Batteries, secondary do

Electrolysis

Paper making, fabries

Batteries, galvanic

Electrolysis .

$$
\text { do }
$$

Liglits, incandescent, special features

res - ....-

Heaters

Railways, systems

Railways, systems, overhead, trolleys -..-

Batteries, galvanic

Electric locomotires

$$
\text { do }
$$

Indicator

Lights, sockets and keys

Motors

$$
\text { do }
$$

Telephones, systems

Telegraphy, circuits and systems

Anti-induction derices

Railwar signals

Lights, are, gravity feed, gear-clutch

Telephones, electric

Scale beams

Batteries, secondary, applications

Railways, systems, overhead, trolleys.

Telegraphy, fire

Liglits, arc, positive feed, clutch

Lights, arc, positive feed, gear-clutch

Lights, are, gravity feed, clutch

Lights, arc, special features

Coin controlled apparatus, weighing .......-

Railways, systems

Insulating raterials

\section{Apparel, cutting}

Telegraphy, railway-car

Cut-outs, thermal

Lights, filaments and carbons

Lights, are, special features

Lights, incandescent, special features - -..-

Lighting, systems do

Clocks, electric

Teleplones, detais

Lights, arc, gravity feed, gear-clutch

Magneto-electric

Motors

Lights, sockets and keys

Clocks, electric -

Electric locomotives

Stone working, drills

Magneto-electric ..

Electric locomotives

Electro-magnets

Clocks, electric

Towers, telegraph poles.

Batteries, secondary do

Telegraphy, relays and sounders

Railway signals

Electrolysis

Railway signals.

Cut-outs, mechanical

Iagneto-electric_

Lights, arc, gravity feed, gear-clutc

Lighting, systems

Clocks, electric

Lights, incandescent, special features

Telegraphy, printing

Dec. 9,1890

Dec. 9,1890

Dec. 9,1890

Dec. 9,1890

Dec. 9,1890

Dec. 9,1890

Dec. 9,1890

Dec. 9,1890

Dec. 9,1890

Sept. 2, 1890

Sejt. 23,1890

Mar. 31, 1891

Apr. 14, 189

Apr. 7, 1891

Feb. 24, 1891

Sept. 16,1890

Scpt. 16, 1890

Sept. 16,1890

Sept. 16,1890

July 29,1890

Aug. 26, 1890

Aug. 5, 1890

June 23, 1891

Nov. 1,1890

Nor. 11, 1890

Dec. 9, 1890

Mar. 10, 1891

dug. 26, 1890

Apr. 14, 1891

Feb. 10, 1891

Dec. 23,1890

Oct. 28, 1890

Dec. 23,1890

June 16, 1891

June 16, 1891

July 15,1890

Feb. 17, 1891

June 23, 1891

Dec. 9,1890

Miay 19, 1891

May 19, 1891

July 8, 1890

Nov. 11, 1890

Aug. 5, 1890

July 29, 1890

June 23, 1891

May 12, 1891

Sept. 30, 1890

June 2, 1891

Mar. 24, 1891

Mar. 24, 189

Mar. 31, 1891

Apr. 28, 1891

Dec. 23, 1890

Dec. 9,1890

July 29, 1890

Nov. 11, 1890

Nov. 25, 1890 


No. $\quad$ NAME. $\quad$ Cidass. $\quad$ Date.

434,892 Scott, G. J

451,881 Scott, G. J

454,882 Scott, (i. J

454,883 Seott, G. J

433,045 Scritiner, C. E

433,046 Seribner, C. E

433,047 Scrituner, C. F

433,424 Scribner, C. E

435,525 Seribner, C. E

435,526 Scribner, C. F

435,527 Scribner, C. E

442,143 Scribner, C. E

442,144 Seribner, C. E

442,145 Scribner, C. E

442,146 Scribner, C. E

452,941 Scribner, C. E

443,464 Sedgwick, $\mathrm{F}$

454,884 Sedgwick, $F$

431,531 Seely, J. A

450,639 Sciberling, J.

432,571 Seibert, $N$

441,258 Seilert, $N$

446,475 Scibert, $N$

447,702 Seibold, $A$. C

448,084 Seibold, A. C

454,187 Sellon, J. S

446,895 Seymour, A. P

441,622 Shaffer, C. H

452,735 Shatter, C. H

439,200 Shanek, H. K

431,235 Slallenberger, O. B

448,676 Shallenberger, O. B

448,677 Shallenberger, O. E

449,001 Shallenberger, $O$. B

449,002 Shallenberger, O. B

449,003 Shallenberger, O. B

434,162 Shallentierger, O. B. and Byllesby, H. M

433187

438,116

438,117

445,907

445,908

451,212

452,619

438,192

453,216

441,030

441,031

441,082

441,033

441,034

454,573

446,477

452,585

448,679

448,680

$466,21:$

431,711

434,034

438,495

415,479

445,654

445,841

446,931

447,208

447,495

448,303

448,681

448,682

448,840

419,709

450,683

451,980

451,981

452,005

452,035

452,036
Shapleigh, M.

shapleigh, Ir

Shaplcigl, W

Slarp, ls. P

Sharp, E. P.

Sharp, E. P

Shaw, A. J
Shawhan, J.

Shawhan, J. F

Sheldon, II. D

Sheldon, W. D

Sheldon, W. I

Sheldon, W. D

sheldon, IV. I

Sherman, IV.

Shivler. H.

Shlaudeman, $\mathrm{F}$

Shorey, G.

Short, S. H

Short, S. H

Short, S. H

Short, S. H

Short, S. H

Short, S. H

Short, S. II

Short, S. H

Short, S. H

Short, S. H

Short, S. H

Short, S. H

Short, S. H

Short, S. H

Short, S. H

Short, S. H

Short, S. H
Shlaudeman,

Short, S. H
Connectors . ... ........ Aug. 19, 1890

Railways, systems, overhead

Motors

June 30,1891

Inductoriums, transformers, rotary ......... June 30, 1891

Cut-outs, thermal .......................... July 29, 1890

Telephones, systems ...................... July 29, 1890

-_._. do _...... July 29, 1890

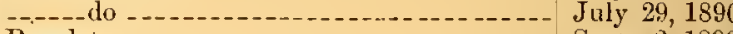

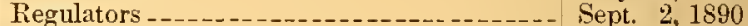

do - . Sept. 2, 1890

-_-_do -..-_-_._._. Sept. 2, 1890

Dec. 9,1890

Dec. 9,1890

Dec. 9,1890

Dec. 9,1890

May 26, 1891

Dac. 23,1890

June 30, 1891

July 1, 1890

Apr. 21, 1891

July 22, 1890

Nov. 25, 1890

Feb. 17, 1891

Mar. 3, 1891

Mar. 10, 1891

June 16, 1891

Feb. 24, 1891

Nov. 25, 1890

May 19, 1891

Oct. 28, 1890

July 1, 1890

Mar. 24, 1891

Mar. 24, 1891

Mar. 24, 1891

Mar. 24, 1891

Mar. 24, 1891

Aug. 12, 1890

July 29, 1890

Oct. 7,1890

Oct. 7,1890

Feb, 3, 1891

Feb. 3, 1891

Apr. 28, 1891

May 19, 1891

Oct. 14,1890

June 2, 1891

Nov. 18, 1890

Nov. 18,1890

Not. ]8, 1840

Nov. 18,1890

Nop. 18, 1890

June 23, 1891

Feb. 17, 1891

May 19, 1891

Mar. 24, 1891

Mar. 24, 1891

Feb. 10, 1891

July 8, 1890

Aug. 12, 1890

Oct. 14,1890

Jan. 27, 1891

Feb. 3, 1891

Feb. 3, 1891

Feb. 24, 1891

Feb. 24, 1891

Mar. 3, 1891

Mar. 17, 1891

Mar. 24, 1891

Mar. 24, 1891

Mar. 24, 1891

Apr. 7, 1891

A ur. 21, 1891

May 12,1891

May 12,1891

May 12, 1891

Nay 12, 1891

May 12, 1891 
$+52,621$

$45 \div, 602$

$+54,00 \mathrm{~s}$

439,904

$49,766^{\circ}$

454,969

436,319

40,271

$41,48:$

43,169

$450,896^{\circ}$

$450,5,5$

$436,6 \pm 0$

445,654

450,260

44,154

$49,866^{\circ}$

45:?

40,10 ;

$+19,829$

439,308

$418,-65$

4t心, 59

438,260

404,315

443,976

454,015

436,023

$412,-34$

$43:, 646$

$436,5+3$

$46,93.5$

413,351

47,166

$44,466^{\circ}$

$453,-4$.

$45 ., 126^{\circ}$

435,047

435,040

135,049

438,118

431,408

436,910

41,591

$4+3,378$

432,57

433,051

437,526

438,375

454,046

454,547

454,543

$43 \pm, 096$

434,097

434,098

$4.4,363$

46,030

446,031

$453,82=2$

437,632

$431, \times 23$

433,425

435,293

45,515

$43-, 259$

439,906

445,358

433,390

449,832

437,666

431,216

431,217

$+31,218$

425, 982

439,042

450,641
Short, S. H

Short, S. H

Short, $\mathrm{S}, \mathrm{H}$

Shubert, H. C

Shuhert, H. C

Sigshee, C. D., Hayward, T. S. and Anderson,

F. S.

Silkman, E. J

silkman, E. J

simpson, I. H

Simpson, J. T

cims, J. C

Sims, II. S

singer, II. $\mathrm{F}$

Singer, W. F

Singer, W. F

Skinner, J. .

-later, IT. II

Slater, IT. II

Elatterr, II. II. M

Slocum, E. I

Slocum, G. S

smith, C. G

Smith, C. R

Smith, C. WV and Lyons, C. J

Smith, F. S

Smith, G. A

Smith, G. II

smith, H. J

Smith, J. R. and Childs, W

Smith, M. H

Snith, R. D.

Smith, R. T

smith, S. II

Smith, S. W

snith, T. C

Smith, T.

Smith, W. A. M. and R. T. F

Smith, WV. F

Smith, IT. F

Smith, W. F

Smith, W.

snee, II

Snelgrove, II

inyder, J. M

Soden, F. H

Spang. H. W

Spang, H. TV

Spang, $\mathrm{H}$. W

Sparrow, F. I

Sperry, 1 . IV

Sperry, A. TV

Sperry, A. II

sperry, E. A

Sperry, E. A

Sperry, E. A

Sperry, E. A

Sperry, E. A

Sperry, E. A

Sperry, E. A

Splitdorf, C. F

Sprague, F. J

Sprague, F. J

Sprague, F. J

Sprague, F. J. and Branth, J. F. S

Eprague, J. J

Sprague, W. B. and Kellogg, C. W

Sprinkmann, $\mathrm{F}$

Stanley, A. F

Stanley, A. F

Stanley, L. T

Stanley, W., jr

Stanley, W. $\mathrm{W} ., \mathrm{j}$

Stanley, W., jr

Stanley, W., jr

Stanley, W., jr

Electric locomotives _._-_-_. May 19, 1891 do

Lights, arc, positise feed, clutch

May 1!, 1891

June !, 1891

Nov. 1,1890

Apr. 7, 1891

June 30,1891

Rheostats

\section{Type-writing}

Alarms

Lamp shades

Telegraphy, circuit closers, thermal

Steering and propulsion

Telegraphy, fire

Telegraphy, circuit closers, thermal

Lights, arc, gravity feed, clutch

Bells

Railway brakes, electric

Batteries, secondary

Telegraphy, fire, alarms and extinguishers :

Railways, systems, overhead, trolleys -

Drop lights and hangers

Wire

Railways, switches, tranıway

Organ hellows

Batteries, galvanic

Lights, arc, special features

Projertiles, primers

Telephones, systems, antomatic

Locomotion

Applications, tools and machines

Railway cars, brakes

Telegraphy, transmitters

$$
\text { do }
$$

Lights, incandescent, special features

'Lighting', systems

Post-office boxes

Lights, sockets an

Lights, sockets and keys

Cut-outs, inechanical

Railway signals

Scale beams

Dampers, automatic

Indicators

Magneto-electric

Lightning rods

Valve-controllers

Conductors

Insulating materials do

Motors

Street cars, steam do

Electric locomotires

Transmission of power

Cut-outs, mechanical

Magneto-electric

Conductors.

Electric locomotipes

Railways, systems, overhead, trolleys ......

Railways, systems, circuits, multiple arc

Railways, systems, overhead

Gearing

Railway signals

Boiler corering

Bells _.

Cut-outs, mechanical

Regulators, alternating

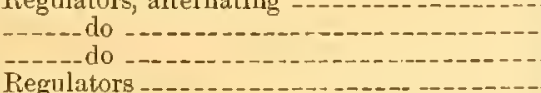

Motors, alternating

Regulators .

Sept. 9, 1890

Mar. 17, 1891

Dec. 2, 1890

Dec. 23,1890

Apr. 21, 1891

Apr. 21, 1891

Sept. 16, 1890

Mar. 24, 1891

Apr. 1t, $189 \mathrm{l}$

Jall. 6, 1891

Apr. 7, 1891

May 26, 1891

Fel, 10, 1891

Apr. 7,1891

Oct. 28, 1890 .

Mar. 24, 1891

Mar. 17, 1891

Oct. 14,1890

Nov. 11, 1890

Dec. 30, 1890

Jnne 23, 1891

Sevt. 9, $18: 0$

Dee. 16,1890

July 22, 1890

Sept. 23,1890

Feb. 24, 1891

Dec. 23, 1890

Feb. 24, 1891

Jan. 13, 1891

June 2, 1891

June 30, 1891

Aug. 26, 1890

Aur. 26, 1890

Aug. 26,1890

Oet. 7, 1890

July 1, 1890

Sept. 23, 1890

Nov. 25, 1890

Mar. 17, 1891

July 22, 1890

July 29,1890

Sept. 30, 1890

July 29, 1890

June 23, 1891

June 23, 1891

June 23, 1891

Aug. 12, 1890

Aug. 12, 1890

Aug. 12, 1890

Aug. 12, 1890

Feb. 10, 1891

Feb. 10, 1891

June 9, 1891

Sept. 30, 1890

July \&, 1890

July 29, 1890

Oct. 14,1890

Jan. 27, 1891

Sept. 30, 1890

Nor. 4, 1890 
443,729 Steele, J. IT

443,730 Steele, J. IV

443,731 Steele, $\hat{J}$. W

443,732 Steele, J. W

435,983 Stephens, W

442,744 Stephenson, $J$

450,846 Stephenson, $J$

454,888 Stephenson, J

449,977 Stern, W. A

451,603 Sterry, T. N

$44 \overline{5}, 721$ Stevens, B. A

454,266

434,682

448,461

443,551

434,163

435,105

445,653

451,282

449,299

451,414

439,792

448,273

448,274

453,871

436,747

451,902

454,669

452,397

435,860

$438,(053$

440,341

447,918

$431,+12$

438,784

453,093

439,441

454503

454,504

439,363

439,364

$439,36.5$

439,366

439,367

432,095

437,946

445,166

436,357

438,564

441,259

447,529

410,071

442,649

150,645

450,646

432,453

442,497

454,087

434,164

433,700

433,701

433,702

433,703

445,207

447,920

447,921

454,622

455,067

455,068

455,069

452,201

435,259

433,544

414,735

455,071

Stewart, R. A

Stillman, F. H

Stilwell, L. B

Stitzel, F

Storer; A

Sturey, I. E

Stout, J. V

Stout, J. V

Stovell, O. II

Stover, J. II

Stover, J. II

Strauss, J. B

Straus, M

Striemer, A

Striemer, A

Strowger, A. B

Studte, H

Siut, J. C. I

Sullivan, J

Swan, 1 .

Swan, A

Swan, A

Swan, $A$

Swart, IT.

Swett, E.

Swift, H. I

Swift, $\mathrm{H}$. I

Sylvester, $P$.

Taintor, G

Talmage. C. H

Talmage, C.

Taylor, J. D

Taylor, W. IV

Taylor, IV. IT

Teague, $\mathrm{F}$

Terry, $\mathrm{C}$.

Tesla, $\mathrm{N}$

Tesla, $\mathrm{N}$

Tesla, $\mathrm{N}$

Tesla, $N$

Tesla, N

Tesla, N

Tesla, N

Tesla, N

Tesla, $\mathrm{N}$

Thew, G
Stevens, W. and Hovey, H. J

Stevens, W. L. and Wescott, E. J

Stitzel, F. and Weinedel, $\mathrm{C}$

St. John, S. H. and Jennings, F. A

Stoecker, H. P

Streichenberg, G. H

Summerskill, C. II

undeen, A. D., Molander, S. B., Anderson,

G. W. and Carlsen, A. M.

sundeen, A. D., Mulander, S. B., Auderson, G. IT. and Carlsen, A. M

Thatcher, E. H

Thobe, A. A. and Embley, TV

Thomas, M. E

Thompson, F. $\mathrm{H}$
Railway gates, automatic, mast

Railways, automatic signals, danger

Railways, automatic signals, trip -

Railway gates, electric

Transmission of power -

Railways, systems, battery

Electric locomotives

Towers, telegraph poles

Applications, general.

Telephones, details

Railway signals, block

Railways, systems, overhead, trolleys

Railways, systems, conduit

Lead pipe making

Telegraphy, lightning arresters

hailmay signals

Station indicators

Patteries, galranic

Bells

Pumps, double piston

Valve-controllers

Lighting, systems

Telegraphy, fire

Connectors

Telegraphy, circuit-closers

Time alarms

Telephones, systems, automatic

Connectors

Telephones, electric

Railways, systems, overhead, trolleys .....

Teleoraphy, circuit closers, thermal

Telephones, systems

Telephones, calls.

Lights, sockets and keys

-.-.--do

$$
\text { do }
$$$$
\text { do }
$$

Railways, systems

Magneto-electric

('ut-r)uts, electro-magnetic

Lights, sockets and keys -

Railways, systems, telpher, solenoid

Sliduble pusher, coin engaged

Cut-outs, electro-magnetic

Heaters

Telephones, calls

Railway signals

(-)

Telegrapliy, printing

Cut-outs, mechanical

Inductoriuins, transformers, stationary -..-

Muturs, alternating

Inductoriums, transformers, stationary

Motors, alternating

Lighting, systems

Magneto-electric

Lighting, systems.

IIotors, alternating

Meters

Lights, incandescent

Railways, elevated

Railwiy signals

Railways, systems, conduit

Merlical and surgical, body-wear

Lights, arc, gravity feed, clutch
Dec. 30,1890

Dec 30,1890

Dec. 30,1890

Dec. 30,1890

Sept. 9, 1890

Dec. 16,1890

A pr. 21, 1891

June 30,1891

Apr. 7, 1891

May 5, 1891

Feb. 3, 1891

June 30, 1891

A ug. 19, 1890

Mar. 17, 1891

Dec. 30,1890

Aug. 12, 1890

Aug. 26, 1890

Feb. 3, 1891

A pr. 28, 1891

Mar. 31, 1891

Apr. 28, 1891

Now. 4, 1890

Mar. 17, 1891

Mar. 17, 1891

June 9, 1891

Sept. 16, 1890

May 5, 1891

June 23, 1891

May 19,1891

Sept. 2, 1890

Oct. 21,1890

Jan. 27, 1891

Mar. 10, 1891

July 1, 1890

Oet. 21,1890

May 26, 1891

Oct. 28,1890

June 23, 1891

June 23, 1891

Oct. 28,1890

Oct. 28, 1890

Oct. 28, 1890

Oct. 28, 1890

Oct. 28, 1890

July 15, 1890

Oct. 7,1890

Mar. 10,1891

sept. 16, 1890

Oct. 14,1890

Nuv. 25,1890

Mar. 3, 1891

Nor. 4, 1890

Dec. 16,1890

Apr. 21, 1891

Apr. 21, 1891

July 15,1890

Dec. 9, 1890

June 16,1891

Aug. 12, 1890

Aug. 5, 1890

Aug. 5, 1890

Aug. 5, 1890

Ang. 5, 1\$90

Jan. 27, 189

Mar. 10, 1891

Mar. 10, 1891

June 23, 1891

June 30,1891

June 30,1891

June 30, 1891

May 12, 1891

A ug. 26,1890

Aug. 5, 1890

Jan. 13, 1891

June 30,1891 
451,20 $431, \pm 14$ 432,581

$+32,651$

432,65

432,653

432,65

4332,6 .

432,6056

$434, \pm 38$

434,48 :

434,530

434,581

434,532

434,961

$43 \overline{7}, 8-0$

$438,20+$

438,650

$438.65 \%$

435,658

40,664

40,663

44,678

$414,9.25$

44,926

$444.2^{2-2}$

44,92

44,929

44,930

44,931

44,946

446,483

447,383

$41 \overline{7}, 384$

44,279

448,280

$4=, 894$

$449,3.36$

449,357

449,836

450,687

451,345

452.951

454,090

454,671

454,672

454,673

454,732

451,890

40,662

440,663

449,71.

435,985

436,677

$435,33:$

441,908

432,657

450.706

440,091

453,435

446,902

451,312

446,037

438,570

454,011

440,934

449,63 ?

432.003

436,025

45.3 .317

454.630

451,960

$44 \overline{7}, 704$

432,098

433,915

443,167

449,767
Thomson, D. P

Thomson, E

Thomson, E

Thomson, E

Thomson, E

Thomson, E

Thomson, $\mathrm{E}$

Thomson, E

Thomson, $\mathrm{E}$

Thomson, $\mathrm{E}$

Thomson, $\mathrm{E}$

Thomson, E

Thomson, $\mathrm{E}$

Thomson,

Thomson, E

Thomson, E

Thomson, $\mathrm{E}$

Thomson, $\mathrm{E}$

Thomson, $\mathrm{E}$

Thomson, $\mathrm{E}$

Thomson, E

Thomson,

Thomson, $\mathrm{E}$

Thomson, E

Thomson, $\mathrm{F}$

Thomson, $\mathrm{E}$

Thonson, $\mathrm{E}$

Thomson, E

Thomson, $\mathrm{E}$

Thomson, E

Thomson, $\mathrm{E}$

Thomson, E

Thomson, E

Thomson, E

Thomson, E

Thumson, E

Thomson, E

Thomson, $\mathrm{E}$

Thomson, $\mathrm{E}$

Thomson, E

Thomson, E

Thomson, F

Thomsnn, E

Thomson, $\mathrm{E}$

Thomson, $\mathbf{E}$

Thomson, E

Thomson, $\mathrm{F}$

Thomson, E

Thomson, E. and Rice. E. W

Thomson, E. and Rice, E. W

Thomson, E. and Rice, E. WV., jr

Thone, F

Thorne, J. and Burr, E. B.

Thury, $R_{-}$

Tiedemann, M. WT

Timmis, I. A

Tinkham, L. B

Tinning, F. T. and W. K. S

Tinning, F. T. and W. K. S

Tirrell, J. P

Tischendoerfer, $\mathrm{F}$

Titus, J. E

Torld, A. E

Tommasi, D. and Therye, C

Tompkins, D. A

Tompkins, $\mathrm{S}$

Torrey, A

Tower, G. A

Townsend, H.

Toye, B. B

Tracy, L. W

Traylor, R. W

Treber, J. MI

Tregurtha, A. T

Tregurtha, A. T

Tregurha, A. T
Drop lights and hangers

Railways, conductor supports and insulators_ July 1, 1890

Railways, srstems, overhead _._._._....... July 22, 1890

Heaters_._........................ July 22, 1890

Magneto-electric .................... July 22, 1890

Heaters _............................ July 22, 1890

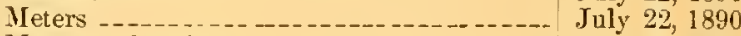

Magneto-electric …….............. July 22, 1890

Heaters_............. July 22, 1890

Motors, alternating -................... Aug. 19, 1890

Transmission of power ............... Aug. 19,1890

Heaters_._. Aug. 19, 1890 do _......... Aug. 19, 1890

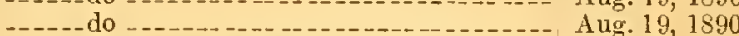

Railways, conductor supports and insulators_ Aug. 26, 1890

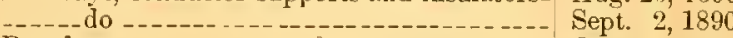

Regulators

Heaters

do

do

Railways, systems, overhead, trolleys

Telegraphy, lightning arresters

Lights, arc, gravity feed, cluteh

Heaters.

-

Cut-outs, thermal

Meters -

Heaters

Railways, conductor supports and insnlators

Lights, are, gravity feed, eluteh

Magneto-electric

Lighting, systems

Meters

Heaters

- do

Railways, systems, overhead

Heaters

Magneto-electric

Inductoriums, transformers, stationary

Telegraphy, lightning arresterss.-..--

-...-. do

Cut-outs, electro-magnetic

Conductors, anti-inductive

Lights, arc, gravity feed, clutch

-

Piheostats

Lights, incandescent, special features

Regulators

Time alarms

Batteries, secondary, applications

Telegraphy, circuit closers -

Telephones, supports -

$$
\text { do }
$$

Cut-outs, electro-magnetic

Regulators

Drop lights and hangers

Railways, systems, telpher, solenoid

Batteries, secondary

Lights, incandescent, special features

Railway signals, cab, sectional rail

Recorders, passenger .

Railway signals

Separators

Telegraphy, multiplex

Lead pipe making

Motors

Telegraphy, relays and sounders

Lights, incandescent, special features

I,ights, sockets and keys

Lights, incandescent, special features.

Oct. 14,1890

Oct. 21, 1890

Oct. 21,1890

Oct. 21, 1890

Nov. 18,1890

Nov. 18,1890

Jan. 13,1891

Jan. 20, 1891

Jan. 20,1891

Jan. 20, 1891

Jan. 20, 1891

Jan. 20, 1891

Jan. 20, 1891

Jan. 20, 1891

Jan. 20, 1591

Feb. 17, 1891

Mar. 3, 1891

Mar. 3, 1891

Mar. 17, 1891

Mar. 17, 1891

Mar. 24, 1891

Mar. 31, 1891

Mar. 31, 1891

Apr. 7, 1891

A pr. 21, 1891

Apr. 28, 1891

May 26, 1891

June 16, 1891

June 23, 1891

Jume 23, 1891

June 23, 1891

June 23, 1891

June 30,1891

Nor. 18, 1890

Nov. 18,1890

Apr. 7, 1891

Sept. 9, 1 \$90

Sept. 16, 1890

Aug. 26, 1890

Dec. 2, 1890

July 22, 1890

Apr. 21, 1891

Nor. 4,1890

June 2, 1891

Feb. 24, 1891

Apr. 28, 1891

Feb. 10, 1891

Oct. 21, 1890

June 16, 1891

Nor. 18, 1890

Mar. 31, 1891

July 8,1890

Sept. 9, 1890

June 2, 1891

June 23, 1891

May 12, 1891

Mar. 3, 1891

July 15, 1890

Aug. 5, 1890

Mar. 10, 1891

Apr. 7,1891 
No.

NAME.
Class.

DATE.
449,921 Tregurtha, A. T

454,238 Tremper, F. E. and Eisenhuth, J. W 440,906

433,554 437,369 436,405 448,698 449,889 439,445 436,961 440,208 416,801 450,304 431,492 431,493 431,494 431,493 431,496 432,345 433,834

434,410 434,684 434,68 .

434,686 434,687

434,816

435,261

435,262

435,263

435,264

436,275

436,276

436,277

437,533

437,534

437,535

438,452

140,976

440,977

443,980

414,185

445,102

447,215

448,561

448,562

448,563

450,541

450,542

450,543

450,54

451,786

452,296

433,835

451,142

447,38

455,075

449,301

$449,30^{\circ}$

450.390

441,861

439,614

442,342

439,213

435,872

$43 \$, 036$

440,780

440,781

441,043

454,532

438,037

$454,58 t$

451,143

$431,08 \mathrm{~s}$

433,58

442,155

445,008

Trott, S

True, L. I

Tschira, A

Tueker, C. A

Turner, D. B

Turner, G. E

Tushingham, F. G. and Dubois, $\mathrm{H}$

Upton, F. R. and Dibble, F. J

Vanderhoef, G. N

$\checkmark$ an de Plancke, $D$, and $G$

$Y$ an Choate, S. F

Van Depoele, C. J

Tan Depoele, C. $J$

Van Depoele, C. J

$\checkmark$ an Depoele, C. J

Vau Depoele, C. J

Van Depoele, C. $J$

Van Deproele, C. J

Van Depoele, C.

Van Depoele, C. J

Tan Depoele, $\mathrm{C}$.

an Depoele, C.

Tan Depoele, C.J

an I lepoele, C. J

Tan Depoele, C. J

Tan Depoele, C. $J$

Tan Deprele, C. J

$\operatorname{Tr}$ an Depoele, C. $J$

Van Depoele, C. J

Van Depoele, C. J

Yan Depoele, C. J

Van Depoele, C.J

Van Depoele, C. J

Yan Depoele, C.J

an Depoele, C. J

Tan Depoele, C. J

an Depoele, C.J

Van Depoele, C. J

Tan Depoele, C. J

an Depoele, C. J

Tan Depoele, C. $J$

Tan Depnele, C. J

Y an Depoele, C.

Yan Depoele, C. J

Yan Depoele, C. J

Van Depoele, C. J

Van Depoele, C. J

Van Depoele, C. J

$V$ an Depoele, C.

Van Depoele, C. J

Tan Deusen, J. I

Van Gestel, J. T

Van Hoesen, G. D

Van Hoevenbergli, H

Van Nuis, C.S

Varley, R., jr

Tan Sant, IT. S. and Eames, E. C

Taughn, B. E

Vaughn, J. II

Veeder, C. H

Verstriete, $\mathrm{E}$

Verstrate, $\mathrm{E}$

Terstriete, E

Verstraete, $\mathrm{E}$

Verstraete, E

Viles, F. T. and Ioung,

Villiers-Stead, F. and Hedgman, E. R

Vincent, C. $\mathrm{H}$

Tining, C. $Y$

Virtue, G. F......
Vogler, $W$.
Lights, incandeseent, special features

Air and gas engines, explosive

Railways, systems, couduit

Ball traps

Alarms -

Lights, arc, positive feed

Cut-outs, mechanieal

Valve eontrollers

Steam valres, throttle

Telegriphy, fire, alarms and extinguishers -

Indicators

Clocks, electrie

Lights, filaments and carbous.-

Motors, reeiprocating

Trans - do -......--

Trado

Eleetric locomotives

Motors, alternating

Railways, ststems, closed comolnit - - .

Railways, systems, converter

$$
\text { do }
$$

Railways, systems

Transmission of power

$$
\text { do }
$$

Railways, eonductor supports and insulators. Railways, systems, closed eonduit

IIetal working, miscellaneous

Transmission of power

$$
\text { do }
$$

Railways, conductor supports and insulators Railways, systems, overhead, trolleys

Electrie locomotives

Car brakes

Railways, systems

Transmission of power

Electrie locomotives

Iotors, alternating

Stone working, drills

Railways, systems, converter

Electric locomotives

Transmission of powe

Electric locomotives.

Jotors reciprocating - -

Motors, alternating

Transmission of power

Telegraphr, keys

Batteries, galvanic

Sack scales.

Telegraphy, printing, wheel

Cut-outs, mechanical

Bells

Annunciators

Fluid delivers

Bells

Telephones, systems

Applications, tocls and machines

Bridges, girder's and columns

Railroads, systems, eonduit_...............

Conduetors, underground

Railways, systems, orerhead, trolleys ....... Rheostats

Telephones, switch-boards

Telephones, systems

Telephones, calls

Annunciators

Applications, tools and machines .........

Railways, conductor supports and insulators

Apr. 7,1891

June 16, 1891

Nov. 18, 1890

Aug. 5, 1890

Sept. 30,1890

Sept. 16,1890

Mar. 24, 1891

Apr. 7, 1891

Oct. 28,1890

Sept. 23,1890

Nov. 11, 1890

Fel. 17,1891

Apr. 1t, 1891

July 1, 1890

July 1,1890

July 1, 1890

July 1,1890

July 1, 1890

July 15, 1890

Aug. 5, 1890

Aug. 12, 1890

Aug. 19, 1890

Aug. 19, 1890

Aug. 19, 1890

Aug. 19,1890

Aug. 19,1890

Ang. 26, 1890

Aug. 26, 1890

Aug. 26, 1890

Ang. 26, 1890

Sept. 9, 1890

Sept. 9, 1890

Sept. 9, 1890

Sept. 30, 1890

Sept. 30,1890

Sept. 30,1890

Oet. $1 t, 1890$

Nov. 18, 1890

Nov. 18, 1890

Dec. 16, 1890

Dec. 30,1890

Jan. 6, 1891

Jan. 20, 1891

Feb. 2t, 1891

Mar. 17, 1891

Mar. 17, 1891

Iar. 17, 1891

Apr. 1t, 1891

Apr. 14, 1891

Apr. 14, 1891

Apr. 14, 1891

May 5, 1891

May 12, 1891

Aug. 5, 1890

Apr. 28, 1891

Mar. 3, 1891

June 30, 1891 
445,108 $439,0.5 .5$ 455,041 40,813 $40.0 \%$ 141,044 44,51 ? 432.500 43.501 436,728 439,584
Fogler, 11

Von Dérschan, A

Ton Kohler, H

Ton Sro, E

Waddell. IT. H

Waddell, M. and Entz, J. B

Wagemann, E

Wagemann, E

TT:agemann, E

TTagemann, E

TIagg, S. R.

Wagner, F. C

ITagnieu, A

IT aite, H. E

Wakefield, W. O

TWalcott, J. E

Walker, G. IY

Wallace, $\mathrm{T}$

Wralter, H. E

Walter, J. H

ITalter, J. M

Tralter, W. C

IV'alter, IV. C

Ward, B. B

Ward, B. B

Ward, B. B

WTard, B. B

Ward, B. B

Ward, J. H. R

Tard, J. H. R

Ward, J. H. R.

WTard, J. H. R

Wardwell, H. I

TTaring, $\mathrm{J}$

TTarner, E. P

Warner, E. P

Tarner, E. P

Warner, E. P

WTarth, A

Warth, A

Warth, A

Warth, A

Trashhurn, G. A

Washhurn, G. A

TIass, D. D

Tratkins, R. I

Waterhouse, A. G

Wratson, J. A

ITebb, G. F

Wehb, J. W

Webher, H. A

TTebber, Tr

TITeed, E. B

Wehrle, J. H

TTehrle, J. H

TTehrle, J. H

TVehrle, J. H

Theller, F. A

TVeller, F. A

WTessel, F. A-

Tressel, F. A

TTessel, F. A.

TFessel, F. A.

TTessel, F. A

Wessel, F.A

Westinghouse, G., j

TTestinghouse, G., jr

TTestinghouse, G., jr $\mathrm{j}$

Weston, E

W'eston, E

Wexton, E
Railways, conductor supports and insulators_. Jan. 20, 1891 Fire arms, locks _.................... Oct. 21, 1890 Clocks, electric . . . June 30, 1891

Telegraphy, circuit closers .............. Nov. 18, 1890 Separators _... Nov. 4, 1890

Railway signals........... .ov. IS, 1890

Electric locomotives _................... Jan. 13, 1891

Magneto-electric ......................... July 15, 1890

Electric locomotives....._._._._._._... July 15, 1890 do do

Paper naking, digester:

Galvanometers

Lights, are, gravity feed, clutch

Batteries, galvanic

Applications, tools and machines

Conductors, lead-covered.

Sept. 16,1890

Oct. 2. 1890

Nov. 11, 1890 June 9,1891 Sept. 23,1890

July 22, 1890 June 16, 1891 Apr. 21, 1891 Sept. '2, 1590 July \&, 1890 Aug. 19, 1590 June 9, 1891 July ], 1890 May 12, 1891 May 2ti, 1891 Jan. 13, 1891 Jau. 13, 1891 Jan. 13, 1891 Mar. 3, 1891

Apr. 7, 1891 Mrar. 31, 1891 Mar. 31, 1891 Mar. 31, 1891 Apr. 28, 1891 June 23, 1891 Dec. 30,1890 Feb. 17,1891

Aug. 5, 1890 Aug. 5, 1890 Mar. 10, 1891

May 26, 1891 Jan. 6, 1891 Jan. 6,1891 Jan. 6, 1891 Jan. 6, 1891

May 5, 1891

May 5, 1891 June 30,1891 Oct. 7, 1890 Apr. 28, 1891 Aug. 26, 1890 June 16, 1891 Mar. 31, 1891 Apr. 21, 1891 July 1, 1890 Nov. 11, 1890 May 19,1891 July 8, 1890 July 8, 1890 Aug. 12,1890 Oct. 7, 1890 Feh. 3, 1891 Nar. 31, 1891 Aug. 19, 1890 Mar. 31, 1891

Apr. 14, 1891 May 19, 1891 June 2, 1891 June 2, 1891 June 2, 1891 Oct. 28,1890 Aug. 12, 1890 Scpt. 9,1890 Apr. 21, 1891 Feh. 10, 1891 A ug. 5, 1890 Nov. 4, 1890 Nov. 11, 1890 
No.

440,290

$+40,291$

$442,8+13$

$+44,803$

444,804

444,805

446,489

$446,+90$

$4+6,+91$

$4+6,492$

$\pm 46,193$

446,494

452,326

442,654

433,751

141,220

$4+1,2: 21$

431,092

431,093

431,094

431,095

453,225

$+333,839$

$418,39 \%$

449,721

$\$ 53,0 \div 0$

$451,88 \pm$

451,885

$45,2,267$

$4+5,59=$

433,91

430,411

435,262

$4+1,210$

411,211

411,212

$4+1,213$

$4+1,21$

$4+1,215$

$\$ 11,216$

$\pm \pm 1,217$

411,218

$4 \pm 1,21$.

$450, \pm 11$

443,507

138,788

$+47,220$

441,45 .

451,190

453,450

434035

445,72

449,188

$43 \div, 394$

446,907

$448, \pm 68$

$436,0.50$

436,952

435, 721

452,351

452,152

439,916

$450,27:$

433,706

436,512

486,513

436,514

437,069

455,268

435,958

442,407

444,900

448,172

448.173

436,030

445,106
NAJE.

Cluass.

D. TE.

Weston, E

Veston, E

Veston, E.

IVeston, E

Veston, F

Weston, F

Westom, H

IVestom,

Vestou, F

Weston, E

Viston, F

Weston, E

Weston, F

Veyburn, E. D

Whartou, W. j

Theatley, S. E

ITheatley, S. E

Wheeler, $\mathrm{F}$

Whecler, $\mathrm{F}^{2}$

II heeler, F

TVheeler, I

Whliceler, F

Wheeler, $\mathrm{F}$. $\mathrm{C}$

Wheeler, G. K

ITheeler, G. K

Wheelcr, S. S

Whecler, S. S

Wheeler, S. is

ITheeler, S. S. and Bradley, C. S

Iilicless, MI

IT'heless, MI

Wheless, M

Whelcss, M

Whelcss, MI

Wheless, M

Wheless, MI

Wheless, MI

Wheless, MI

Wheless, M

Whelcss, $\mathrm{Nl}$

IThelcss, MI

Whipple, F.

IVhitcomb, A. IV

White, A. C

White, A. C

White, L. R.

White, L. R. S

White, L. R. S

White, O. B

White, O.

White, W. li

Whitlock, F

Whitney, E. R

Whittier, T. P

Whittingham, G. H

Whittingham, G. H

IVhittingham, G. H

Whitingham, G. H

Whittleser, J. T

Wickes, R. O

Widdifield, W. P. and Bowman, A. H

Miegand, S. I

Wriegaud, S. L

Wiegand, S. L

Wiesebrock, F. IV

Wiesebrock, F. W

Wightman, N1. J

Wightman, M. J

Wightman, M. J

Wightman, M. J

Wightman, M.

Wilder, E. R

Wiley, E. C
Galvanometers

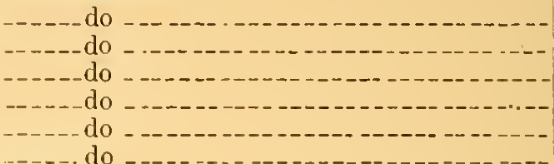

Rheostats

Galvanometers

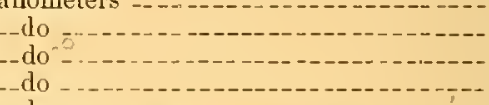

-do

Applications, tools and machines

Street cars, steam

Railways, systems, circnits, multiple arc

Railways, systems, conduit

Raiimays, systems, overhead

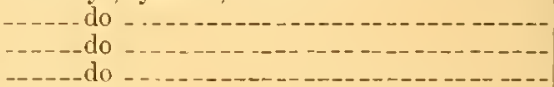

Connectol"s

Railways, srstems, overliead, trolley

Insulators

Railways, conductor supports and insulators.

Migneto-electric

Regulators

Railways, systems _...

Railways, systems, circuits, multiple arc ....

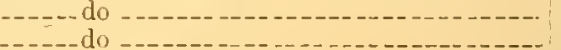
do

Railways, systems, conduit ...............

Railways, sytems, circuits, multiple arc Railways, systems, conduit.

Electriı lucomotives _._-_....---

Railways, sfstems, closed conduit

Railways, systems, closed conduit

Railways, systems, closed conduit ..........

Railways, systems, circuits _...........

Railwav's, svstems, circuits, multiple arc -

Miotors

Lights, are, gravity feed, gear-clutch _......

Telegraphy, lightning arresters..........

Telcphoncs, svistens

Igniting derices, automatic .

Cut-out:, mechauical

Lights, sockcts and keys

Drup lights and hangers

Lights, arc, gravily feed, cluteh

Cut-outs, mechanical

Cut-outs; thermal

Elevators

Lights, incandeseent, special features

Batterics, secundar 5

$\overline{-10 t u r s}$

Cut-outs, therma

Railways, systems, orerhead trollers ---

Telegrawhy, relays and sounders

Conductors

Telephones, magnetic

Telcphones, electric -

Telephones, relars and repeaters

Optics, divtanue inutruments

Telethermometers

Motors

Railwars, systems, circuits, multiple arc...-

Mritors

Railways, systems, orerbead, trolleys.....

Alarms, burglar

Railway signals
Metal working, miscellaneous

Cut-outs, thermal

Nov. 11,1890

Nov. 11,1890

Dec. 16,1890

Jan. 13, 1891

Jan. 13, 1891

Jan. 13, 1891

Feb. 17, 1891

Feb. 17, 1891

Feb. 17, 1891

Feb. 17, 1891

Feb. 17, 1891

Feh. 17, 1891

May 12, 1891

Dec. 16,1890

Aug. 5, 1890

Nov. 25, 1890

Nov. 25, 1890

July 1, 1890

July 1, 1890

July 1, 1890

July 1, 1890

June 2, 1891

Aug. 5, 1890

Mar. 17, 1891

Apr. 7, 1891

Мay 26, 1891

Мay 5, 1891

May 5,1891

June 30,1891

Mar. 17, 1891

Ang. 5, 1890

Sept. 2, 1890

Oct. 1t, 1890

Nov. 11, 1890

Nov. 25, 1890

Nuv. 25, 1890

Nov. 25, 1890

Nov. 25, 1890

Nov. 25, 1890

Nov, 25,1890

Nor. 25, 1890

Nov. 25, 1890

Nov. 25, 1890

Nov. 25, $1 \times 90$

Apr. 14, 1591

Dec. 30,1890

Oct. 21, 1890

Fcb. 24, 189

Noy. 25, 1890

Apr. 28, 1891

June 2, 1891

Aug. 12, 1890

lieb. 3, 1891

Mir. 31, 1891

Oct. 14,1890

Feh. 24, 1891

Mar. 17, 1891

Sept. 9, 1890 Sept. 2?, 1890

Oct. 21, 1890

M:uy 12, 1891

N1:1 1:2, 1891

Nov. 4,1890

A pr. 14, 1s91

Aug. 5, 1890

Sept. 16, 1890

Sept. 16, 1890

Sept 16,1890

Sept. 23, 1890

Junc 30, 1891

Sept. 9, 1n90

Dec. 9, 1890

Jan. 20, 1891 
No

NAME.

Classs.

DATE.

46,313

433.920

$433,9: 1$

433,92:-

449,990

$437,27=$

452.250

44,594

450,955

451,586

451,557

452.091

452,510

452,125

433,331

436,281

447,85

434,746

450,810

414,374

434,899

446,214

433,505

414,144

445,348

445,399

445,400

413,401

433,504

450,690

435,340

431,134

433,557

435,668

454,541

445,790

436,964

440,334

$\$ 11,966$

48,792

$+38,211$

46,826

448,703

452,299

46,199

454,924

431,681

437,387

441,999

442,501

443,693

438,590

434,224

433,334

415.295

436,516

450,923

439,047

439,550

440,216

445,306

444,477

$446,5(1) 2$

$4.50,086$

455,057

$447,8.53$

446,544

436,941

$4: 4,166$

434,167

43,168

484,169

434,170

484,371

433,430

443,155

451,101
Wiler, E. C

Wiles, G. L. and Acheson, E. G

Wiles, G. L. and Acheson, E. G

Wiler, G. L. and Acheson, E. G

Wilheln, C. W

Wilking, F. and Wüller, $\mathrm{H}$

Williams, C. D

Willıams, H. B

Williams, J. A

Williams, J. B

Williams, J. B

Williams, J. B

Williams, J. B

Williams, J. B

Williams, J. T

Williams, J, T

Williams, P. G

Williams, R. E

Willms, C

Wilson, C. E

Tilson, C. E

Wilsou, C. $\mathrm{H}$

ITilson, J. C

ITilson, J. C

Wilson, J. C

Tilson, J. C

Wilson, J. C

Wilson, J. C

Wilson, J. C. and Noyes, B. J

ITilson, T.S

Wilson, WT. H

Tinkler, C. F

Winkler, C. F

Winkler, C. F

Winkley, E. E

Winton, H. D

Tirt, C

IVirt, $\mathrm{C}$

Tirirt, C

Tirt, $\mathrm{H}$.

Wirt, H. C

Virt. H. C

Wiseman, $A$

Wood, F. B

Wood, J. J

Wnod, J. J

Wood, J. J

Wood, J, J

Wood, J. J

IToods, G. T

Woodward, $\mathrm{H}$

W'ondward, J. H

Wollensak,

Wollensak, J. F. and Gill, W. E

Woolley, L. G

Toolf, A. E

Woolf, A. E

Woolcott, C. Y. and Wilmot, A.

ITright, A. P

Wright, E. G

ITright, G. WT

IV right, G. W

Wright, T. F and Wilson, $\mathrm{E}$. G

Wright, II

Wright, W. H.

Wurts, A

IVurts, A

Wiurts, A

Wurts, A

Wurts, A

TWurts, A

Wurts, $\mathrm{A}$. and Baldwin, $\mathrm{O} . \mathrm{H}$

II'yckoff; A. B

Young, C. G.
Railway signals

Conductors.

$$
\text { do }
$$

Railway signals, sectional rail

Batteries, secondary, applications

Medical and surgical, body-wear

Recorders

Regulators

Conductors

do

do

do

Railways, systems, telpher, solenoid -do

Medical and surgical, body-wear

$$
\text { do }
$$

Batteries, galvanic

Cut-outs, mechanical

Conductors, underground

Telegraphy, fire

- do do

Thermostat

Telegraphy, fire

Electrodes

Railway signals

Magneto-electric

-----do

Valve contiollers

Insulators

Magneto-electric

Rheostats ..

Galvanometers

Railways, conductor supports and insulators

Lights, incandescent, special features

Railways, conductor supports and insulators

Clocks, electric

Telegraphy, fire

Annunciators

Lights, sockets and keys

Machines for winding

Telegraphy, lightning arresters

Meters

Cut-outs, mechanical

Lighting, systems -

Batteries, secondary

Electrodes

Bells

Batteries, galvanic

Inductoriums, induction coils

Lights, sockets and key

Batteries, secondary

Alarms

Conductors

Miscellaneous compositions

Telegraplyy, circuit closers

Lights, arc, positive feed, gear-clutch...--.-

Anti-induction devices

Batteries, galvanic

Railways, systems, overhead

Telegraphy, lightning arrester

Cot-outs, thermal

- - - - do - _._.......

Telegraphy, lightning arresters

- do - do - - do

Lighting, systems

Lights, arc, gravity feed, gear-clutch

Feb. 10, 1891

Aug. 5,1890

Aug. 5, 1890

Aug. 5, 1890

Apr. 7,1891

Sept. 30, 1890

May 12,1891

Mar. 3, 1891

A pr. 21, 1891

May 5, 1891

May 5, 1891

May 12, 1891

May 19,1891

May 19, 1891

July 29, 1890

Sept. 9, 1890

Mar. 10, 1891

Aug. 19, 1890

Apr. 21, 1891

Jan. 6, 1891

Aug. 19,-1890

Feb. 10, 1891

A ug. 5, 1890

Jall. 6, 1891

Mar. 17, 1891

Mar. 17, 1891

Mar. 17, 1891

Mar. 17, 1891

Aug. 5, 1890

Apr. 21, 1891

Aug. 26, 1890

July 1,1890

Aug. 5, 1890

Oct. 21, 1890

June 23, 1891

Feb. 3, 1891

Sept. 23, 1890

Nov. 11,1890

Jan. 20, 1891

Mar. 2t, 1891

Oct. 1t, 1890

Feb. 17, 1891

Mar. 24, 1891

May 12, 1891

Fel. 10,1891

June 30, 1891

July 8, 1890

Sept. 30,1890

Dec. 2, 1890

Dec. 9,1590

Dec. 30,1890

Oct. 14,1590

Aug. 12, 1890

July 29, 1890

Jan. 27, 1891

Sept. 16, 1890

Apr. 21, 1891

Oet. 21, 1890

Nov. 4, 1890

Nov. 11, 1890

Jan. 27, 1891

Jau. 13, 1891

Feb. 17, 1891

June 30, 1891

June 30, 1891

Mar. 10, 1891 
ALPHABETICAL INDEX-ELECTRICITY.

\begin{tabular}{|c|c|c|c|}
\hline No. & NAME. & Class. & DATE. \\
\hline 453,281 & Young, C. G..- & Lighting, systems .. & June 2,1891 \\
\hline 442,162 & Young, H. H .. & Conductors, underground _ & Dec. 9,1890 \\
\hline 437,668 & Young, J & Telegraphy, fire, alarms and extinguisher & Sept. 30,1890 \\
\hline 449,923 & Youngs, E. F & Type-writing & Apr. 7,1891 \\
\hline 451,548 & Zagelmeyer, L _---_-- & Telegraphy, circuit closers, thermal ... & May 5,1891 \\
\hline 454,245 & Ziegler, J. O_...... & Railway signals & June 16,1891 \\
\hline 449,890 & Zingsen, F. W & Electro-deposition & Apr. 7,1891 \\
\hline 433,758 & Zipernowsky, C. and Dèri, MI _............... & Transmission of power & Aug. 5, 1890 \\
\hline 439,459 & Zipernowsky, C., Dèri, M. and Bláthy, O. T-- & Motors, alternating & Oet. 28,1890 \\
\hline 445,583 & Zipernowsky, C & Electric locomotives & Feb. 3, 1891 \\
\hline 452,056 & Zipernowsky, C $\ldots$ & Heaters & May 12,1891 \\
\hline 434,076 & Zöpke, F. G. C....... & 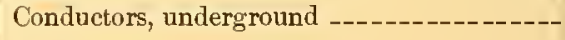 & Ang. 12, 1890 \\
\hline
\end{tabular}




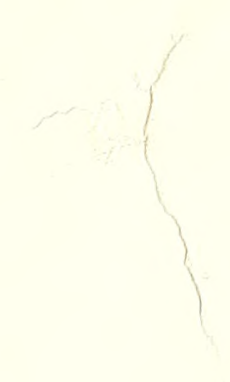




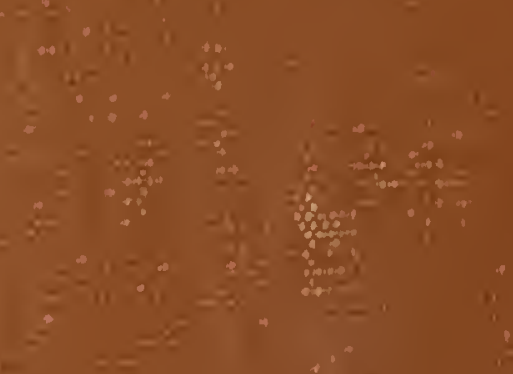

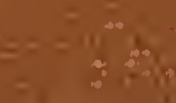

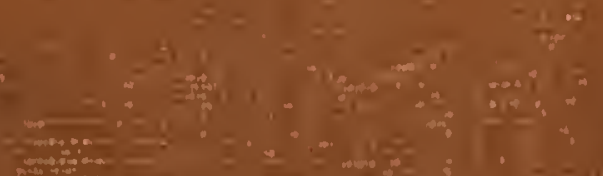

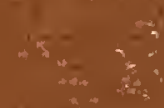
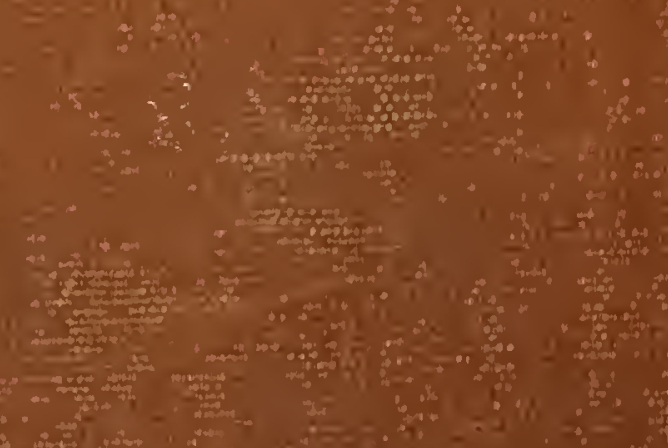